\title{
Diffusion-weighted magnetic resonance imaging at ultra-high field
}

Citation for published version (APA):

Lagos Fritz, F. (2021). Diffusion-weighted magnetic resonance imaging at ultra-high field: From ex vivo to in vivo imaging of the human brain. [Doctoral Thesis, Maastricht University]. Ipskamp Printing BV. https://doi.org/10.26481/dis.20210111flf

Document status and date:

Published: 01/01/2021

DOI:

10.26481/dis.20210111flf

Document Version:

Publisher's PDF, also known as Version of record

\section{Please check the document version of this publication:}

- A submitted manuscript is the version of the article upon submission and before peer-review. There can be important differences between the submitted version and the official published version of record.

People interested in the research are advised to contact the author for the final version of the publication, or visit the DOI to the publisher's website.

- The final author version and the galley proof are versions of the publication after peer review.

- The final published version features the final layout of the paper including the volume, issue and page numbers.

Link to publication

\footnotetext{
General rights rights.

- You may freely distribute the URL identifying the publication in the public portal. please follow below link for the End User Agreement:

www.umlib.nl/taverne-license

Take down policy

If you believe that this document breaches copyright please contact us at:

repository@maastrichtuniversity.nl

providing details and we will investigate your claim.
}

Copyright and moral rights for the publications made accessible in the public portal are retained by the authors and/or other copyright owners and it is a condition of accessing publications that users recognise and abide by the legal requirements associated with these

- Users may download and print one copy of any publication from the public portal for the purpose of private study or research.

- You may not further distribute the material or use it for any profit-making activity or commercial gain

If the publication is distributed under the terms of Article $25 \mathrm{fa}$ of the Dutch Copyright Act, indicated by the "Taverne" license above, 
Doctoral thesis

\section{DIFFUSION-WEIGHTED MAGNETIC RESONANCE IMAGING AT ULTRA-HIGH FIELD: FROM EX VIVO TO IN VIVO IMAGING OF THE HUMAN BRAIN}

Francisco J. Fritz

2020

Faculty of Psychology \& Neuroscience, Maastricht University 



\title{
DIFFUSION-WEIGHTED MAGNETIC RESONANCE IMAGING AT ULTRA-HIGH FIELD: FROM EX VIVO TO IN VIVO IMAGING OF THE HUMAN BRAIN
}

\author{
Dissertation \\ To obtain the degree of Doctor at Maastricht University, \\ on the authority of the Rector Magnificus, Prof. Dr. Rianne M. Letschert, \\ in accordance with the decision of the Board of Deans, \\ to be defended in public \\ on 11 of January, 2021, at 16:00 hours
}

by

Francisco J. Fritz 


\title{
Promotor
}

Dr. Alard Roebroeck

Prof.dr. Rainer Goebel

\section{Copromotors}

Prof.dr. Benedikt A. Poser

\author{
Assessment Committee \\ Prof.dr. E. Formisano (Chair) \\ Dr. F. De Martino \\ Dr. D. Ivanov \\ Prof.dr. D.G. Norris, Donders Institute for Brain, Cognition and \\ Behaviour, Radboud University, The Netherlands \\ Prof.dr. N. Weiskopf, Max Planck Institute for Human Cognitive \\ and Brain Sciences, Leipzig, Germany
}

(c) Francisco J. Fritz, Hamburg 2020.

All rights reserved. No part of this publication may be reproduced, stored in a retrieval system or transmitted in any form or by any means, electronic, mechanical, photocopying, recording or otherwise, without prior written permission of the author.

This work was supported by an European Research Council (ERC) Starting Grant (MULTICONNECT, \#639938)

Cover Nicole L. Fritz, 2020

Production Ipskamp Printing

ISBN 978-94-6421-176-4 
A mi madre, mi padre, mis hermanas y hermano... y a mi pequeño amado Jack.

-o-0-0-0-0-0-0-0-0-0-0-0-0-0-0-0-0-0-0-0-0-0-0-0-

Yo me mantuve alejado de mi puesto durante años

Me dediqué a viajar, a cambiar impresiones con mis interlocutores

Me dediqué a dormir;

Pero las escenas vividas en épocas anteriores se hacían presentes en mi memoria.

Durante el baile yo pensaba en cosas absurdas:

Pensaba en unas lechugas vistas el día anterior Al pasar delante de la cocina,

Pensaba un sinnúmero de cosas fantásticas relacionadas con mi familia;

Entretanto el barco ya había entrado al río

Se abría paso a través de un banco de medusas.

Aquellas escenas fotográficas afectaban mi espíritu,

Me obligaban a encerrarme en mi camarote;

Comía a la fuerza, me rebelaba contra mí mismo,

Constituía un peligro permanente a bordo

Puesto que en cualquier momento podía salir con un contrasentido.

"Notas de viaje" - Nicanor Parra 



\section{Contents}

1 Notations 1

2 Introduction $\quad 5$

3 kT-dSTEAM: Ultra-high resolution and multi-shell diffusion MRI of intact ex vivo human brains at $9.4 \mathrm{~T}$

4 Human whole-brain diffusion MRI at $450 \mu \mathrm{m}$ post mortem with $\mathrm{k}_{\mathrm{T}^{-}}$ dSSFP and a specialised 9.4 T RF-coil

5 Comprehensive analysis and characterisation of the steady-state signal in the STEAM sequence

6 MESMERISED: Super-accelerated 7 T STEAM imaging for quantitative multi-contrast and diffusion MRI

7 General Overview and Discussion $\quad 145$

8 Valorisation $\quad 159$

9 Publication list 163

$\begin{array}{lr}10 \text { Curriculum Vitae } & 169\end{array}$

$\begin{array}{ll}\text { Bibliography } & 171\end{array}$ 



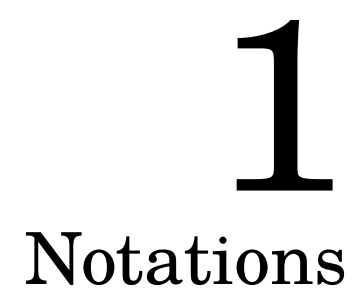


For simplification purposes, this thesis uses the Tensor Notation and the Einstein summation convention in some parts, such as the Introduction and General Discussion Chapters. As defined from reference [177]: A tensor of order $M$ is a linear function of $N$ covectors and $M-N$ vectors into the real numbers, which is independent of the reference frame in which those vectors (and covectors) are calculated. Then, a Tensor is defined by its order and its indexes. The order is defined as the dimensional size (visualised by the number of indexes which can be super-scripted or sub-scripted) and the indexes are referred to the vector basis where the Tensor is defined. In this thesis only Tensors of order 0 (scalars), order 1 (vectors) and order 2 (matrices) in the spatial (or Cartesian) space (i.e. $\mathrm{x}, \mathrm{y}$ and $\mathrm{z}$ coordinates in the laboratory frame) are employed (index $i$ for order 1 Tensors, indexes $(i, j)$ for order 2 Tensors and indexes (i,j,k) for order 3 Tensors). The following notations are defined:

1. Scalar (order 0 Tensor): Any Tensor with no-index (e.g. number or algebraic variable like 1 or $\mathrm{x}$ ) or any order Tensor with valued indexes (e.g. if $\mathbf{C}_{\mathrm{i}}{ }^{\mathrm{j}}$ is a order 2 Tensor, then $\mathrm{C}_{1}{ }^{1}$ is a scalar).

2. Vector (row-vector or simply vector, order 1 Tensor): $\mathbf{C}_{\mathbf{i}}=\left[C_{0}, C_{1}, C_{2}, \ldots, C_{n}\right]$

3. Covector (column-vector, order 1 Tensor): $\mathbf{C}^{\mathbf{i}}=\left[\begin{array}{c}C^{0} \\ C^{1} \\ C^{2} \\ \ldots \\ C^{n}\end{array}\right]$

4. n-component of a (co)vector: For a vector $\mathbf{C}_{\mathbf{i}}$, its n-component is defined as $\mathrm{C}_{\mathrm{n}}$, while for a covector $\mathbf{C}^{\mathbf{i}}$, its $\mathrm{n}$-component is defined as $\mathbf{C}^{\mathrm{n}}$ (which are scalars).

5. Basis vector: $\mathbf{e}_{\mathbf{i}}$ (defined in bold). For example, in spatial space the basis vector is defined as $\mathbf{x}_{\mathbf{i}}$ (for $\mathrm{i}=1: 3$ they represent the $\mathrm{x}-, \mathrm{y}$ - and z- coordinates). Each component is written as the n-component of a (co)vector as above, but in bold.

6. Einstein Summation Convention (ESC): Any (co)vector $\mathbf{C}_{\mathbf{i}}\left(\mathbf{C}^{\mathbf{i}}\right)$ can be written as sum of its components with its vector (covector) basis: $\mathbf{C}_{\mathbf{i}}$ $=\mathrm{C}_{0} \mathbf{e}^{\mathbf{0}}+\mathrm{C}_{1} \mathbf{e}^{\mathbf{1}}+\mathrm{C}_{2} \mathbf{e}^{\mathbf{2}}+\ldots=\sum C_{i} \mathbf{e}^{\mathbf{i}}$ ( $\sum C^{i} \mathbf{e}_{\mathbf{i}}$ in case of a covector). 
Therefore, the ESC simplifies this notation by multiplying a scalar vector (covector) with a basis covector (vector): $\mathrm{C}_{\mathrm{i}} \mathbf{e}^{\mathrm{i}}\left(\mathrm{C}^{\mathrm{i}} \mathbf{e}_{\mathbf{i}}\right)$.

7. Scalar-vector components of a Tensor: For any Tensor of order 1 (vector or covector), it can be decomposed by its scalar vector (covector) and basis covector (vector): $\mathbf{C}_{\mathbf{i}}=\mathrm{C}_{\mathrm{i}} \mathbf{e}^{\mathrm{i}}\left(\mathbf{C}^{\mathrm{i}}=\mathrm{C}^{\mathrm{i}} \mathbf{e}_{\mathbf{i}}\right)$.

8. Dot product: Defined as the product of a vector and covector (i.e. $\mathbf{u}_{\mathbf{i}}$ and $\mathbf{v}^{\mathbf{i}}$ ) on the same vector basis: $\mathbf{u}_{\mathbf{i}} \mathbf{v}^{\mathbf{i}}=\mathbf{u}^{\mathrm{i}} \mathbf{e}_{\mathbf{i}} \mathbf{v}_{\mathbf{i}} \mathbf{e}^{\mathbf{i}}=u^{\mathrm{i}} \mathbf{v}_{\mathbf{i}}$ (the multiplication of the basis with itself is 1 ).

9. Two-dimensional matrix (order 2 Tensor): $\mathbf{C}^{i}{ }_{j}=\left[\begin{array}{ccccc}C_{0}^{0} & C_{1}^{0} & C_{2}^{0} & \ldots & C_{n}^{0} \\ C_{0}^{1} & C_{1}^{1} & C_{2}^{1} & \ldots & C_{n}^{1} \\ C_{0}^{2} & C_{1}^{2} & C_{2}^{2} & \ldots & C_{n}^{2} \\ \ldots & \ldots & \ldots & \ldots & \ldots \\ C_{0}^{n} & C_{1}^{n} & C_{2}^{n} & \ldots & C_{n}^{n}\end{array}\right]$

10. Matrix transpose: $\mathbf{C}^{j}{ }_{i}=\left[\begin{array}{ccccc}C_{0}^{0} & C_{0}^{1} & C_{0}^{2} & \ldots & C_{0}^{n} \\ C_{1}^{0} & C_{1}^{1} & C_{1}^{2} & \ldots & C_{1}^{n} \\ C_{2}^{0} & C_{2}^{1} & C_{2}^{2} & \ldots & C_{2}^{n} \\ \ldots & \ldots & \ldots & \ldots & \ldots \\ C_{n}^{0} & C_{n}^{1} & C_{n}^{2} & \ldots & C_{n}^{n}\end{array}\right]$

11. Cross product: defined as a summation over permutations of components of two vectors (or covectors) with different indexes (i.e. $\mathbf{u}^{\mathbf{j}}$ and $\left.\mathbf{v}^{\mathbf{k}}\right): \epsilon_{j k}^{i} u^{j} v^{k} \mathbf{e}_{i}$ where $\epsilon^{i}{ }_{j k}$ is defined as the Levi-Civita symbol (e.g. in the Cartesian three dimensional space, it is equal to 1 if $(\mathrm{i}, \mathrm{j}, \mathrm{k})=$ $(1,2,3),(2,3,1)$ and $(3,1,2)$; to -1 if $(\mathrm{i}, \mathrm{j}, \mathrm{k})=(3,2,1),(1,3,2)$ and $(2,1,3)$ and to 0 if $i=j, j=k$ or $k=1$ ).

12. Partial derivative: It is defined as the differentiation of each (function) component of the Tensor with respect the differential variable. For example, the partial derivative of a Tensor $\mathbf{u}^{i}$ in function of time is written as $\partial_{t} \mathbf{u}^{i}$ and defined as: $\frac{\partial u^{i}(t)}{\partial t} \mathbf{e}_{i}$. (only if the basis vector is not dependent on the variable being differentiated).

For consistency and for a clearer understanding of the terms, the indexes are evaluated with the coordinates instead of numbers. For example, a covector $\mathbf{C}^{\mathrm{i}}$ with basis in the Cartesian space has scalar components $\mathrm{C}^{\mathrm{x}}$, 
$\mathrm{C}^{\mathrm{y}}$ and $\mathrm{C}^{\mathrm{z}}$ (instead of $\mathrm{C}^{1}, \mathrm{C}^{2}$ and $\mathrm{C}^{3}$ as commonly used. This is also valid for vectors). 


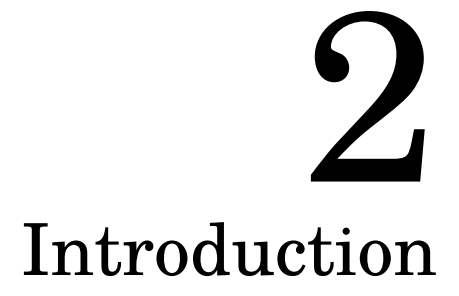




\subsection{Magnetic Resonance Imaging}

This section provides an introduction to the principles of Nuclear Magnetic Resonance (NMR) and Magnetic Resonance Imaging (MRI), its application for quantitative MRI (qMRI) and diffusion MRI (dMRI) as they pertain to the subject matter of this thesis. It will review the pulsed-gradient spinecho (PGSE), steady-state free precession (SSFP) and the stimulated echo acquisition method (STEAM) sequences mostly applied in a dMRI context, which much of the work shown in this thesis is built upon.

\subsubsection{Nuclear magnetic resonance, NMR}

NMR (and hence MRI) employs the combination of a static magnetic field $\left(\mathbf{B}_{0}{ }^{i}\right)^{1}$ and oscillating magnetic fields, by using radiofrequency (RF-) pulses $\left(\mathbf{B}_{1}{ }^{i}\right)$, to generate a resonance effect on the magnetic 1/2-spin nuclei [30, 169]. For the purpose of imaging living tissue, this is generally applied to the protons $\left({ }^{1} \mathrm{H}\right)$ in the water molecules $\left(\mathrm{H}_{2} \mathrm{O}\right)$ due to its abundance in the living organism (e.g. almost $60 \%$ in human adults [224]). In principle, NMR/MRI can also target any other spin-1/2 nuclei (whose abundance compared to water can be negligibly small). For example, the most common element after water is Potassium $\left({ }^{31} \mathrm{P}\right)$ with a relative abundance of $0.14 \%$ and relative sensitivity of $9.3 \%$ compared to water.

The magnetic effect of $\mathbf{B}_{0}{ }^{i}$ and $\mathbf{B}_{1}{ }^{i}$ on nuclei can be described from a quantum perspective or using a semi-classical approach. The former is beyond the scope of this thesis, but the interested reader is referred to reviews and books that can be found elsewhere (e.g. in references [44, 201]). In the semi-classical perspective, the interaction of $\mathbf{B}_{0}{ }^{i}$ on the nuclear magnetic spin $\left(\mathbf{J}^{i}\right)$ results in a torque on it $\left(\tau^{\mathrm{i}}\right)$ (Figure 2.1A), given by the Larmor equation (Equation 2.1 left). The resulting angular frequency from $\mathbf{J}^{i}$ is called the Larmor frequency $\left(\omega_{0}\right)$, which is proportional to the gyromagnetic ratio $\left.(\gamma \text {, in [time }]^{-1}[\text { magnetic flux density }]^{-1}\right)$ and the $\mathbf{B}_{0}{ }^{i}$ strength (Equation 2.1 right).

$$
\tau^{i}=\partial_{t} \mathbf{J}^{i}=\gamma\left(\epsilon^{i}{ }_{j k} \mathbf{J}^{j} \mathbf{B}_{0}{ }^{k} \mathbf{e}_{i}\right) \longrightarrow \omega_{0}=-\gamma \sqrt{\mathbf{B}_{0 i} \mathbf{B}_{0}{ }^{i}} .
$$

\footnotetext{
${ }^{1}$ In this introduction chapter and wherever relevant throughout this thesis, Tensorial notation and the Einstein summation conventions will be used. Please see Chapter 1 for the meaning of these notations/conventions.
} 
where $\epsilon^{i}{ }_{j k}$ is the Levi-Civita symbol and $\mathbf{e}_{\mathrm{i}}$ the coordinate frame system (i.e. for $\mathrm{x}, \mathrm{y}$ and $\mathrm{z}$ axis, here on referred as $\mathbf{x}^{\mathrm{i}}$ ).

In a macro-system with a large number of nuclei $\left(\mathrm{N}_{\mathrm{s}}\right)$, at thermal equilibrium at temperature $\mathrm{T}_{\mathrm{s}}$, the influence of $\mathbf{B}_{0}{ }^{i}$ creates a bulk magnetisation $\left(\mathbf{M}^{i}\right.$ ) parallel to $\mathbf{B}_{0}{ }^{i}$ (pointing along the longitudinal axis) ${ }^{2}$. Its transverse components $\left(\mathrm{M}^{\mathrm{x}}\right.$ and $\left.\mathrm{M}^{\mathrm{y}}\right)$ are considered null (Figure 2.1B). Its magnitude is proportional to the temperature of the sample, the Planck's constant $(\hbar)$, the Boltzmann constant $\left(\mathrm{K}_{\mathrm{B}}\right)$ and the strength of $\mathbf{B}_{0}{ }^{i}$ :

$$
\sqrt{\mathbf{M}_{i} \mathbf{M}^{i}}=\frac{\gamma^{2} \hbar^{2} \sqrt{\mathbf{B}_{0 i} \mathbf{B}_{0}{ }^{i}} N_{s}}{4 K_{B} T_{s}} \quad K_{B}=1.38 \cdot 10^{-23} \mathrm{~J} / \mathbf{K} \quad, \quad \hbar=6.63 \cdot 10^{34} \mathbf{J s}
$$

A

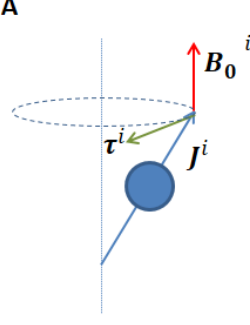

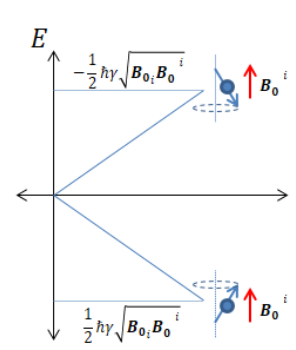

B

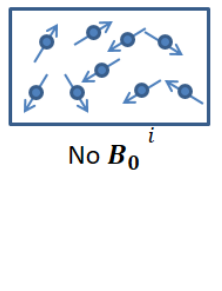

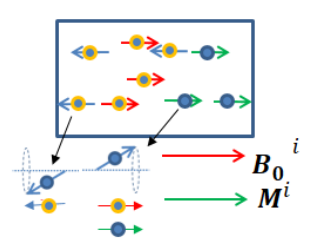

Figure 2.1: NMR representation: (A) Semi-classical representation of the effective torque on a nucleus produced by the interaction between $\mathbf{J}^{i}$ and $\mathbf{B}_{0}{ }^{i}$ (left) which can results in spins aligned parallel (ground state) or antiparallel (excited state) with respect to $\mathbf{B}_{0}{ }^{i}$ (right). (B) On the macro-scale, without the influence of $\mathbf{B}_{0}{ }^{i}$, the $\mathbf{J}^{i}$ of nuclei are distributed randomly and, on average, no bulk magnetisation is created (left). But when $\mathbf{B}_{0}{ }^{i}$ is applied, a higher number of (precessing, as shown in A) spins aligns parallel to this field (red and green arrows) and the remaining (precessing spins) are aligned antiparallel (light blue arrows) [44]. An equal number of spins aligned parallel and antiparallel cancel magnetically each other (yellow spheres), while the remaining spins (blue spheres - green arrows) generate a bulk magnetisation (main green arrow, $\mathbf{M}^{i}$ ) parallel to the main field. These are the spins which can be manipulated by the RF pulses.

\footnotetext{
${ }^{2}$ This is conventionally written as $\mathbf{M}^{\mathrm{z}}$, because the alignment of $\mathbf{B}_{0}{ }^{i}$ is by convention along the z-axis
} 
$\mathbf{M}^{i}$ is manipulated by the application of the RF pulse, characterised by Equation 2.3, where the RF pulse is fully characterised by its initial phase angle $(\varphi)$, pulse envelope function (or shape, $B_{1}{ }^{e}(t)$ ) and the Larmor frequency $\omega_{0}$.

$$
\begin{array}{r}
\mathbf{B}_{1}{ }^{i}(t)=B_{1}{ }^{e}(t) \mathbf{E}^{i}{ }_{j}\left(\omega_{0}, t, \varphi\right) \mathbf{x}^{j} \\
\text { with } \quad \mathbf{E}^{i}{ }_{j}\left(\omega_{0}, t, \varphi\right)=\left[\begin{array}{ccc}
\cos \left(\omega_{0} t+\varphi\right) & -\sin \left(\omega_{0} t+\varphi\right) & 0 \\
\cos \left(\omega_{0} t+\varphi\right) & \sin \left(\omega_{0} t+\varphi\right) & 0 \\
0 & 0 & 0
\end{array}\right]
\end{array}
$$

When the RF pulse is applied in the process known as excitation, $\mathbf{M}^{i}$ is tilted with respect to the z-axis at a specific angle, also known as the excitation flip angle $(\alpha)$. This is calculated as:

$$
\alpha=\int_{0}^{T_{d u r}} B_{1}{ }^{e}(t) d t \quad \rightarrow \quad \mathbf{M}_{\alpha+}^{i}=\mathbf{R}_{j}^{i}(\varphi) \mathbf{M}^{j}
$$

where $\mathrm{T}_{\text {dur }}$ is the duration of the RF pulse, $\mathbf{M}_{\alpha+}^{i}$ the resulting magnetisation after $\mathrm{T}_{\mathrm{dur}}$ and $\mathbf{R}_{\mathrm{j}}^{\mathrm{i}}$ represents the rotation matrix with angle $\alpha$ with respect to the axis defined by $\varphi$. After $\mathrm{T}_{\text {dur }}$, the nuclei excited by $\mathbf{B}_{1}{ }^{i}$ return to the initial magnetisation (e.g. ground state [36]) by two relaxation processes. These relaxation processes are energy transfers that occur simultaneously: (1) between the spins and the medium, referred to as spin-lattice relaxation, which occurs at the tissue-specific longitudinal relaxation time $\mathrm{T}_{1}$ (called as $\mathrm{T}_{1}$ relaxation process) [29, 93], and (2) between the spins, referred to as spin-spin relaxation, that occurs at the transverse relaxation time $\mathrm{T}_{2}$ (called as $\mathrm{T}_{2}$ relaxation process) [29]. However, in NMR experiment the measured $\mathrm{T}_{2}$ is often shorter. This is principally due to the effect of inhomogeneities in the main magnetic field (e.g. susceptibility-induced field distortions produced by other tissues or materials within $\mathbf{B}_{0}{ }^{i}$ ). The resulting $\mathrm{T}_{2}$ relaxation is referred as effective- $\mathrm{T}_{2}$ or $\mathrm{T}_{2} *$ (a good summary of this concept can be found in reference [50]). These relaxation times are dependent on $\mathbf{B}_{0}{ }^{i}$ and the medium surrounding the excited nucleus. For example, at a magnetic strength of $1.5 \mathrm{~T}$, the ${ }^{1} \mathrm{H}$ proton in water as a $\mathrm{T}_{1} / \mathrm{T}_{2}$ of $4.0 \mathrm{~s} / 2.0 \mathrm{~s}$, respectively, and corresponding ratio of $0.25 \mathrm{~s} / 0.07 \mathrm{~s}$ in fat $[33,190]$. Furthermore, when the magnetic field strength increases, $\mathrm{T}_{1}$ generally increases, while $\mathrm{T}_{2}$ always decreases $[29,33]$. This $\mathrm{T}_{2}$ relaxation process is manifested by the release of photons, referred as the free induction decay (FID) signal [36]. This is one of the MR signals that can 
be acquired and it can be processed either as a temporal signal or spatial image (see section 2.1.2). The mathematical description that summarises the FID signal from an RF excitation pulse until complete relaxation was introduced by Felix Bloch in 1946, known as the Bloch equation [29]. In 1956, Torrey introduced two additional terms in order to take two further processes into account: the effect of diffusion (D) and flow velocity (v) [204]. This results in the so-called Bloch-Torrey equation:

$$
\partial_{t} \mathbf{M}^{i}=\gamma\left(\epsilon^{i}{ }_{j k} \mathbf{M}^{j} \mathbf{B}_{0}{ }^{k} \mathbf{e}_{i}\right)+\mathbf{R}^{i}{ }_{j} \mathbf{M}^{j}+\mathbf{C}^{i}-\nabla^{i}\left(\mathbf{v}^{i}{ }_{j} \mathbf{M}^{j}\right)+\nabla^{i}\left(\mathbf{D}^{i}{ }_{j}\left(\nabla^{j} \mathbf{M}^{j}\right)\right)
$$

where:

$$
\mathbf{R}_{j}^{i}=\left[\begin{array}{ccc}
\frac{-1}{T_{2}} & 0 & 0 \\
0 & \frac{-1}{T_{2}} & 0 \\
0 & 0 & \frac{-1}{T_{1}}
\end{array}\right] \quad \text { and } \quad \mathbf{C}^{i}=\left[\begin{array}{c}
0 \\
0 \\
\frac{M_{0}}{T_{1}}
\end{array}\right]
$$

where $\mathrm{M}_{0}$ corresponds to the total bulk magnetisation magnitude prior to the RF pulse excitation or after a relaxation process.

\subsubsection{Magnetic Resonance Imaging, MRI}

The MR signal needs to be spatially encoded in order to obtain an interpretive spatial image. To achieve this, the MRI system contains specialised hardware like the gradient coils (hereafter gradients, $\mathbf{G}^{i}$ ) which can spatially vary (e.g. linearly) the main magnetic field strength $\left(\sqrt{\mathbf{B}_{0 i} \mathbf{B}_{0}{ }^{i}}\right)$ through the entire scanner bore. One example is the slice selection gradient $\left(G^{z}\right)$, which varies $\mathbf{B}^{i}$ (in this case, its z-component $B_{z}$ ) linearly along the z-axis (Equation 2.8). From Equation 2.1, this linear variation of $\mathbf{B}^{i}$ depends on the strength of the slice selection gradient resulting in a variation of the resonance frequencies around $\omega_{0}\left(\gamma\left|\mathrm{G}^{\mathrm{z}}\right|=\delta_{\omega}\right)$.

$$
B^{z}=B_{0}{ }^{z}+\left(z-z_{0}\right) G^{z} \rightarrow \omega(z)=-\gamma B^{z}=\omega_{0}+\left(z-z_{0}\right) \delta_{\omega}
$$

When the excitation RF pulse is applied at mean frequency $\omega_{0}$ and with a specific bandwidth (BW), only the nuclei precessing at this (range of) frequencies contained in an specific slice thickness $\mathrm{z}_{1}\left(\mathrm{BW}=\left(\mathrm{z}_{1}-\mathrm{z}_{0}\right) \delta_{\omega}\right)$ will be excited. This forms the principle of selectively exciting a slice (Figure $2.2 \mathrm{~A})$. The use of gradients is also extended to the other two spatial dimen- 


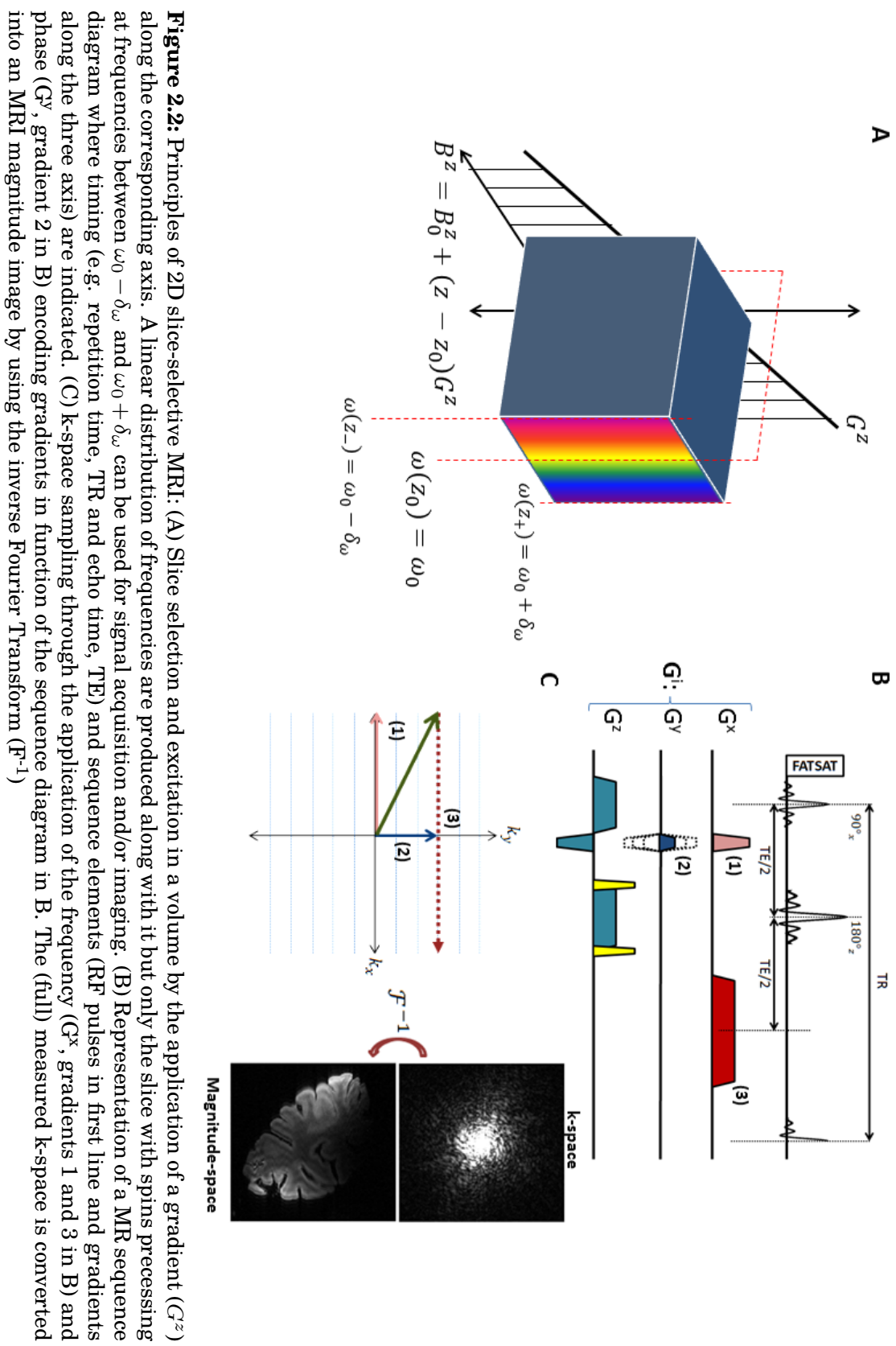


sions, along the $\mathrm{x}$ - and $\mathrm{y}$-axes ${ }^{3}$ called the frequency and phase encoding gradients in slice-selective $2 \mathrm{D}$ imaging. The $\mathrm{x}$ - and $\mathrm{y}$ - gradients, in contrast to the slice selection gradient, are not applied simultaneously with an RF pulse (e.g. Figure 2.2B), but serve the purpose of spatially perturbing the frequencies of the already excited nuclei. The frequency encoding gradient ( $\mathrm{G}^{\mathrm{x}}$ in Figure 2.2B), as its name indicates, perturbs the frequencies while the signal is being detected by the receiver. This gradient is also called readout gradient (in Figure 2.2B, gradient (3)). The phase encoding gradient ( $\mathrm{G}^{\mathrm{y}}$ in Figure 2.2B), on the other hand, perturbs the phase of the excited nuclei (in Figure 2.2B, gradient (2)). The application of those gradients for spatial frequency and phase tagging, results in a spatiallyfrequency-encoded received signal described as follows:

$$
\mathbf{k}^{i}=\frac{\gamma}{2 \pi} \int_{t} \mathbf{G}^{i}(t) d t \quad \rightarrow \quad S\left(\mathbf{k}^{i}\right)=\int_{y} \int_{x} \rho\left(\mathbf{x}^{i}\right) \exp \left(-2 \pi i \mathbf{x}_{i} \mathbf{k}^{i}\right) d \mathbf{x}^{i}
$$

where $\mathbf{k}^{\mathrm{i}}$ is defined as the k-vector (resulting green arrow and dashed arrow (3) in Figure 2.2C), a position vector of a frequency-space called the $\mathrm{k}$-space $\left(\mathrm{S}\left(\mathbf{k}^{\mathrm{i}}\right)\right.$ in Figure $\left.2.2 \mathrm{C}\right)$ and $\rho\left(\mathbf{x}^{\mathrm{i}}\right)$ is the proton density at the spatial position $\mathbf{x}^{\mathrm{i}}$. When the k-space is fully sampled, applying the inverse Fourier transform on it can transform it in a spatially encoded signal. Acquiring the magnitude of the signal in this space results in the conventional MR image (Figure 2.2C, right) [135].

Sampling the complete k-space takes a considerable amount of time compared to the duration of the FID signal after the excitation pulse. To make the signal last longer, and in turn be affected by the spatial-encoding gradients, and be recorded afterwards, it is necessary to reverse the dephasing of the spin's nuclei during $\mathrm{T}_{2}$. This can be done by using a gradient reversal [230, 77] or by applying an extra RF pulse [100]. This reversal effect makes the spins rephase again, generating a new MR signal called the echo [100] (Figure 2.3). The formation of this echo occurs at a time called echo time (TE), which is equal to two times the time between the excitation of $\mathbf{M}^{i}$ and when the corresponding refocusing element ( $R F$ pulse or gradient) is applied. If the echo is created by using a gradient reversal, this echo is known as the gradient-recalled echo (GRE, e.g. see reference [77]) (Figure $2.3 \mathrm{~A})$. But if the echo is created by the extra $\mathrm{RF}$ pulse, called refocusing $\mathrm{RF}$ pulse, then it is known as the spin-echo (SE) [100] (Figure 2.3B). This rephasing approach is not only limited to one echo, but can be extended

\footnotetext{
${ }^{3}$ The commonly used axis labels are arbitrary, but customarily the remaining two axes of $\mathbf{G}^{i}$ must be orthogonal to the axis used for the slice-selective gradient
} 
to several echoes (or stimulated echo, section 2.2.2) which can be formed by applying a train of RF pulses and/or gradients reversals. However, the echoes created by the GRE or SE approaches are not the same in presence of magnetic field inhomogeneities. Whereas the use of a gradient reversal does not eliminate the extra dephasing produced by the magnetic inhomogeneity, the use of a refocusing $R F$ pulse does. As a consequence, the echo created by the GRE approach is $\mathrm{T}_{2}{ }^{*}$-weighted while the one created by the $\mathrm{RF}$ pulse is $\mathrm{T}_{2}$-weighted [77, 50]. 

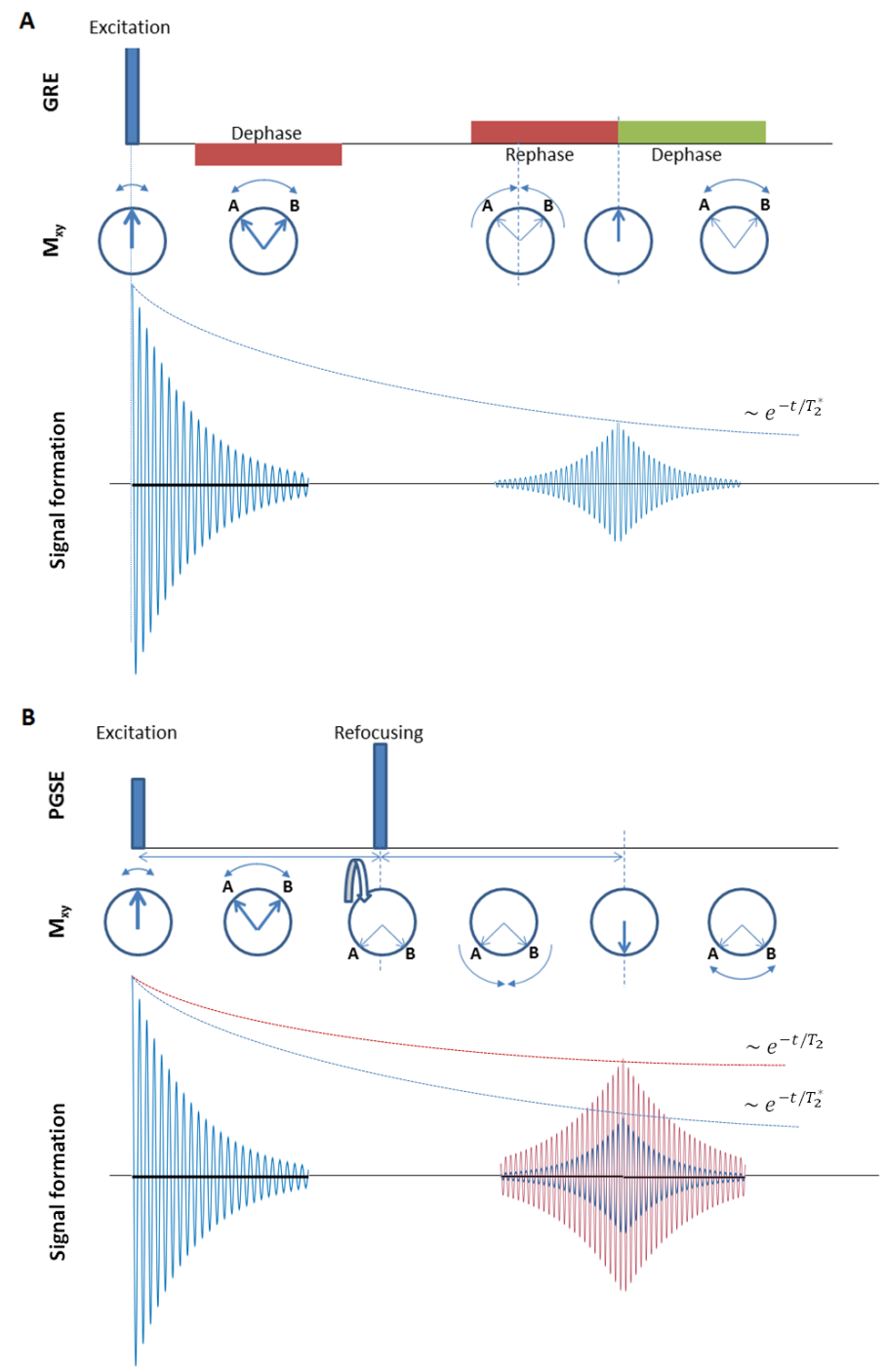

Figure 2.3: Echo formation. Two approaches can be used to recover the signal created by the excitation pulse (the free induction decay signal, FID) in a longer period of time. (A) The first approach is the gradient-reversal echo (GRE), which uses a rephasing gradient with a total momentum (= gradient amplitude $\cdot$ duration) equal to the dephase gradient used after the excitation pulse. The resulting echo at the echo time (TE) is $\mathrm{T}_{2}{ }^{*}$-weighted, because the gradients do not nullify the contribution of any magnetic inhomogeneity. (B) The second approach is the spin echo (SE, or PGSE in Figure) approach, which uses a (refocusing) RF pulse, which rotates the transverse plane in such way the spins converge back without affecting their motion. In contrast to the GRE approach, the resulting echo is $\mathrm{T}_{2}$-weighted (superimposed red curves) because the pulse does nullify the contribution of any magnetic inhomogeneity. 
The sequential combination between RF pulses, echoes and gradients defines the concept of an MR pulse sequence. The intensity (or magnitude) of the resulting echo in a MR sequence is obtained by solving Equation 2.6 and calculating the resulting transverse component $\left(\left|\mathrm{M}^{\mathrm{xy}}\right|\right)$ at TE. It is evident that the echo signal will be dependent on (or weighted by) $\mathrm{T}_{2}, \mathrm{~T}_{1}$, and other physical factors.

\subsection{Diffusion-weighted MRI (dMRI)}

As shown in Equation 2.6, the echo signal is also affected by the diffusion of the nuclei during the MR sequence. By adding and manipulating gradients, it is possible to make the resulting signal sensitive to diffusion. To understand how to obtain anatomical information from MR images weighted by this phenomenon, it is relevant to introduce the physics of diffusion (subsection 2.2.1), and how to acquire and interpret diffusion-weighted MR images (subsection 2.2.2) through three diffusion-weighted sequences namely dPGSE, dSTEAM and dSSFP.

\subsubsection{The Physics of Diffusion}

Diffusion is defined as a physical phenomena in which the molecules move randomly in a system (or between systems) due to their vibrational-thermal energy $[76,56]$. This phenomena could occur either between different particles (e.g iodine molecules diffuse in water) or between same particles (e.g. water molecules diffusing between each other). The latter scenario is called self-diffusion [76]. In both cases, the corresponding (self-)diffusivity (D, in (length) ${ }^{2}(\text { time })^{-1}$ ) can be obtained by using Fick's second law [81]. While in the first case the diffusivity is determined by (and dependent on) the concentration of the solute molecules $\left(C_{\text {sol }}\right)$, the second case is dependent on the spatial probability distribution of the molecules, which is referred as the propagator $\left(P\left(\mathbf{r}^{i}, \mathbf{r}^{i}, \tau\right)\right)$ [124] (Equation 2.10, Figure 2.4). In this work, the focus will be on self-diffusivity phenomena.

$$
\text { Self-diffusivity: } \quad \partial_{t} P\left(\mathbf{r}^{i}, \mathbf{r}^{\mathbf{p}^{i}}, \tau\right)=\nabla\left(D \nabla P\left(\mathbf{r}^{i}, \mathbf{r}^{\boldsymbol{s}^{i}}, \tau\right)\right)
$$

The propagator describes the probability of a molecule from an initial position $\left(\mathbf{r}^{i}\right)$ be located in another position $\left(\mathbf{r}^{, i}\right)$ at a specific time lapse 
A
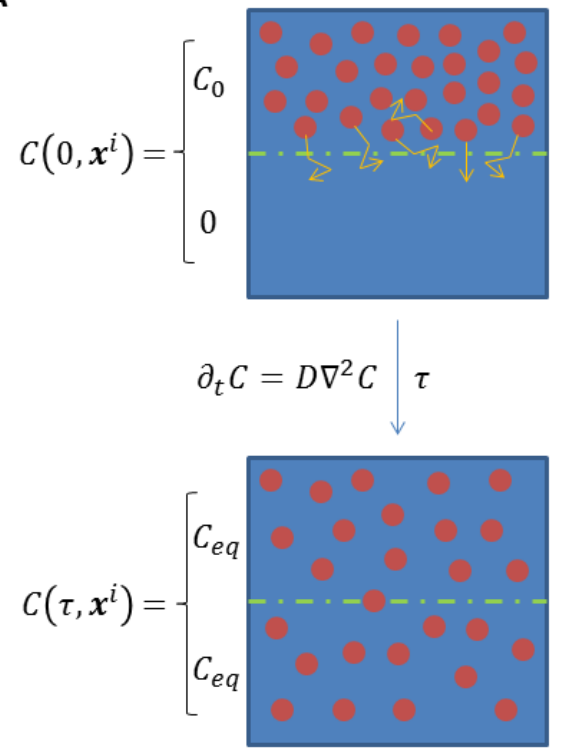

B
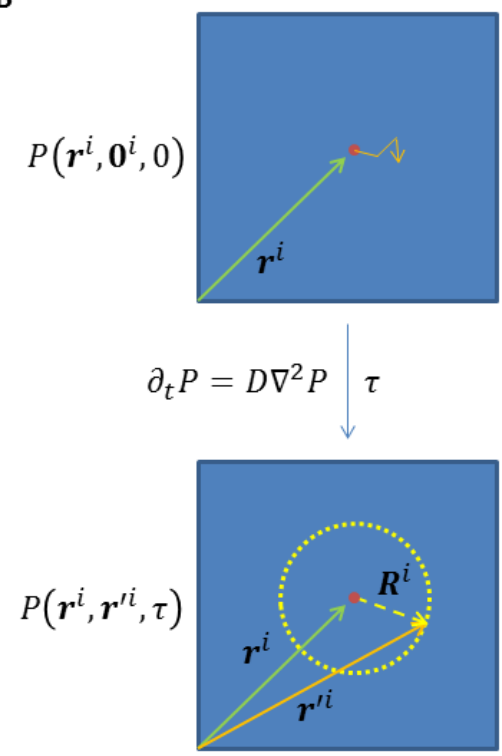

Figure 2.4: Graphical illustration of the difference between diffusion and self-diffusion. While diffusion (A) is produced by a change of concentration of the solute molecules, selfdiffusion (B) is produced due to the thermal motion and interaction between molecules. Nevertheless, in both cases, Fick's second equation is used to estimate the corresponding (self)diffusivity.

( $\tau$ ) (Figure 2.4B). Fick's second law equation applied to estimate the selfdiffusivity is also called the Fokker-Planck equation [172].

A single-molecule self-diffusing cannot be studied but instead it is plausible to estimate the mean self-diffusivity of many molecules. This is obtained by specifying the density distribution of the molecules across the medium $\left(\rho\left(\mathbf{r}^{i}\right)\right)$. With this information, the mean self-diffusivity can be estimated by the ensemble average propagator, also referred as the probability density function (PDF) of the molecules displacement $\left(\mathbf{R}^{i}=\mathbf{r}^{i}-\mathbf{r}^{i}\right.$, Equation 2.11).

$$
\operatorname{PDF}\left(\mathbf{R}^{i}, \tau\right)=\int P\left(\mathbf{r}^{i}, \mathbf{r}^{, i}, \tau\right) \rho\left(\mathbf{r}^{i}\right) d \mathbf{r}^{i}
$$

The corresponding solution(s) (using Equation 2.11 in Equation 2.10) is (are) dependent on the system boundaries (e.g. rigid walls and initial 
conditions) and timescale [56, 168, 55]. For example, in an homogeneous media $\left(\rho\left(\mathbf{r}^{i}\right)=\rho\right.$ ) with isotropic self-diffusivity (D), the resulting PDF is a probabilistic Gaussian function with mean $\mu=\sqrt{\mathbf{R}_{i} \mathbf{R}^{i}}$ and variance $\sigma^{2}=6 D \tau$. This probabilistic function is known as the Einstein diffusion equation and its variance is referred as the Einstein length [56, 76]. When the self-diffusivity becomes orientationally dependent, or anisotropic $\left(D=\mathbf{D}^{i}{ }_{j}\right)$, the result is extended as shown in Equation 2.12.

$$
\operatorname{PDF}\left(\mathbf{R}^{i}, \tau\right)=\frac{1}{\sqrt{\operatorname{det}\left(\mathbf{D}^{i}{ }_{j}\right)(4 \pi \tau)^{3}}} \exp \left(-\frac{\mathbf{R}_{i} \mathbf{D}^{i}{ }_{j} \mathbf{R}^{j}}{4 \tau}\right)
$$

Where $\mathbf{D}^{i}{ }_{j}$ is the diffusion matrix (or rank-2 tensor) and $\operatorname{det}\left(\mathbf{D}^{i}{ }_{j}\right)$ is the determinant. It is possible to determine the directions (and corresponding magnitudes) where the self-diffusivities are predominant by decomposing $\mathbf{D}^{i}{ }_{j}$ in its corresponding eigenvectors $\left(\mathbf{e}_{i} \mathrm{~s}\right.$ in $\left.\mathbf{V}^{j}{ }_{i}=\left(\mathbf{e}_{1}, \mathbf{e}_{2}, \mathbf{e}_{3}\right)\right)$ and eigenvalues $\left(\lambda_{i} \sin \Lambda_{i}^{i}=\operatorname{diag}\left(\lambda_{1}, \lambda_{2}, \lambda_{3}\right)\right)$ :

$$
\mathbf{D}^{i}{ }_{j}=\mathbf{V}^{j}{ }_{i} \Lambda_{i}^{i} \mathbf{V}^{i}{ }_{j}
$$

This Equation is largely used to describe the self-diffusivity of water molecules in well-ordered structures, but it can be used in other biological systems. However, additional assumptions must be considered. Some of them are time or geometry dependent on the diffusion process. For example, Figure 2.5 shows the estimation of the self-diffusivity at three time points $\left(\tau_{1}, \tau_{2}\right.$ and $\tau_{3}$ ) for two systems: free and restricted in a cylinder. In a short time pe$\operatorname{riod}\left(\tau_{1}\right)$, for both cases, the self-diffusivity is approximately isotropic and equal in magnitude. Then, it would be difficult to determine if the water molecules are diffusing freely or not. However, the motion of the molecules start to be affected by the barriers of the cylinder for longer times $\left(\tau_{2}\right.$ and $\tau_{3}$ ). This results in a lower diffusivity along the cross section while along the cylinder it diffuses as if it is still free [127] ${ }^{4}$.

\footnotetext{
${ }^{4}$ However, other processes make the self-diffusivity along the cylinder lower in comparison to free diffusivity, as shown in the reference [127]
} 
A

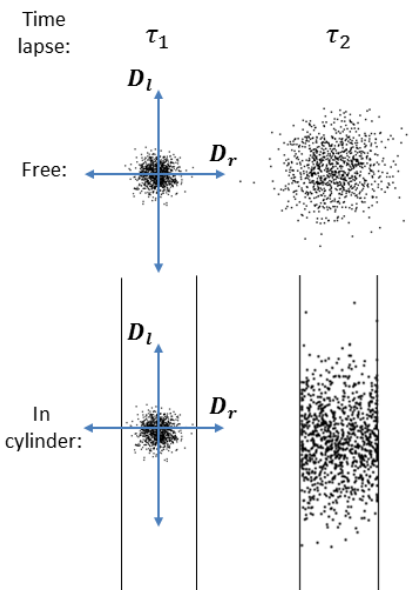

B
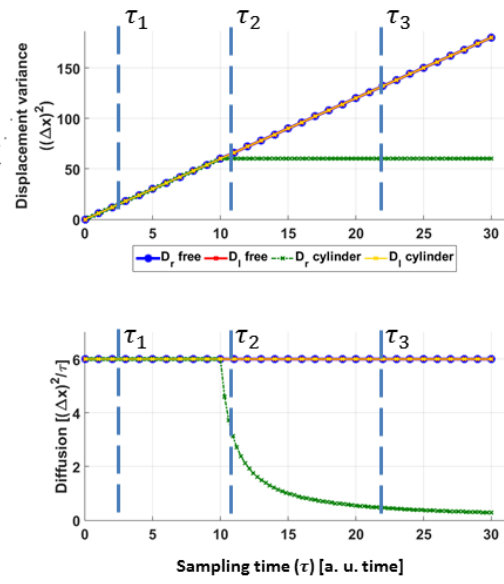

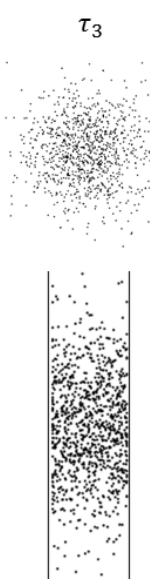

Figure 2.5: (A) Graphical illustration of the evolution of the self-diffusivity in two different systems (top: free, bottom: cylinder) and in three time periods $\left(\tau_{i}\right)$ measured radially $\left(\mathrm{D}_{r}\right)$ and longitudinally $\left(\mathrm{D}_{l}\right)$. (B) Curves representing the increment of variance with respect time (top) and the resulting estimation of self-diffusivity (bottom) by using the Einstein length equation in both systems for the three different time periods shown in (A). It is clear that the self-diffusivity decreases when it is restricted and it reaches an asymptote for long times.

\subsubsection{Diffusion-weighted MR sequences: dPGSE, dSTEAM and dSSFP}

Diffusion-weighted MR sequences (dMRI) are capable of making the echo signal sensitive to water self-diffusion phenomena, as explained previously. The most common sequences used for this purpose are the (diffusion-weighted) pulsed-gradient spin-echo (dPGSE) ${ }^{5}$, the diffusion-weighted stimulated echo acquisition method (dSTEAM) and the diffusion-weighted steady-state free precession (dSSFP) sequences.

SE and dPGSE sequence

The SE sequence [100] is an MR sequence which uses two RF pulses to create the echo signal (as previously mentioned in section 2.1.2, and shown in Figure 2.3): the excitation pulse ( $\alpha_{1}$, with phase along the $\mathrm{x}$ or $\mathrm{y}$ axis)

\footnotetext{
${ }^{5}$ Strictly, the pulsed-gradient form of the spin-echo sequence (PGSE) is diffusion-weighted by definition. Here, we add the 'd' to PGSE for consistency of notation
} 
and the refocusing pulse ( $\alpha_{2}$, with phase along the $\mathrm{z}$ axis). This sequence diagram is shown in Figure 2.6.

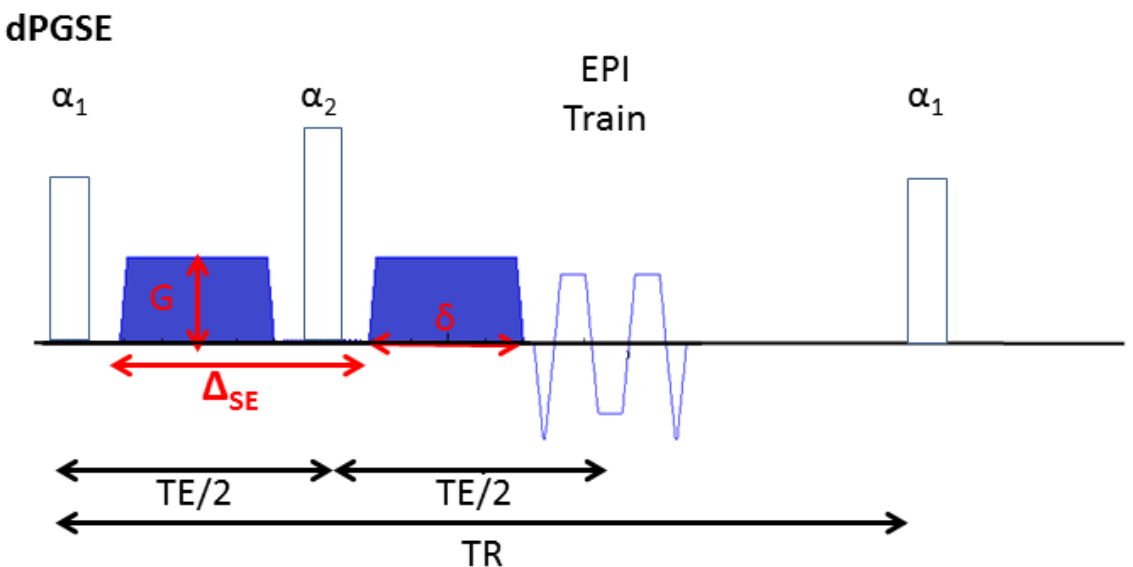

Figure 2.6: dPGSE sequence: Sequence diagram where the excitation $\left(\alpha_{1}\right)$ and refocusing $\left(\alpha_{2}\right) \mathrm{RF}$ pulses are indicated. At time TE is when the echo is formed and commonly encoded by the readout gradient train (EPI train). The time between excitation pulses is known as repetition time (TR). Diffusion gradients (in blue) are added to convert the SE sequence to a dPGSE sequence. The corresponding diffusion parameters are diffusion time $(\Delta)$, diffusion gradient duration $(\delta)$ and diffusion gradient amplitude $(G)$ are indicated.

Solving the corresponding Bloch equation for this sequence (Equation 2.6 without the diffusion and flow components), the spin-echo signal at time $\mathrm{TE}$ is defined as:

$$
S_{S E}\left(\alpha_{i}, T R, T E, T_{1}, T_{2}\right)=S_{0}\left(1-\exp \left(\frac{-T R}{T_{1}}\right)\right) \sin \left(\alpha_{1}\right) \sin \left(\frac{\alpha_{2}}{2}\right)^{2} \exp \left(\frac{-T E}{T_{2}}\right)
$$

where $S_{0}$ represents the unweighted (or total) signal. This signal is maximised when the combination of $\pi / 2-\pi \mathrm{rad}$ flip angles (for $\alpha_{1}$ and $\alpha_{2}$, respectively) are used.

The dPGSE sequence was the first implemented dMRI sequence. It was created in 1965 by Stejskal and Tanner [192]. They introduced the diffusion gradients, a couple of gradients (applied along the same gradient axis) both placed symmetrically before and after the refocusing RF pulse (Figure 2.6, blue gradients). As mentioned in the last section, it is clear that the use (and/or addition) of any gradient will perturb the distribution 
of frequencies, resulting in a signal change. It was estimated that the effect of the diffusion gradients (with any shape, $\left.\mathbf{G}(t)^{i}\right)$ in the signal at echo time (TE) is calculated as follows:

$$
\ln \left(\frac{S(\tau=T E)}{S_{0}}\right)=\left(-\gamma^{2} D\left[\int_{0}^{\tau} \mathbf{F}(t)_{i} \mathbf{F}(t)^{i} d t-4 \mathbf{f}_{i}\left(\int_{\tau}^{\prime \tau} \mathbf{F}(t) d t\right)^{i}+4 \mathbf{f}_{i} \mathbf{f}^{i}\left(\tau-\tau^{\prime}\right)\right]\right)
$$

$$
\text { with } \mathbf{f}^{i}=\mathbf{F}\left(\tau^{\prime}\right)^{i} \text { and } \mathbf{F}(t)^{i}=\int_{0}^{t} \mathbf{G}\left(t^{\prime}\right)^{i} d t^{\prime}
$$

In the case the diffusion gradients have a rectangular shape $\left(\mathbf{G}(t)^{i}=\mathbf{G}^{i}\right)$ with equal diffusion gradient duration ( $\delta$ ), amplitude $\mathrm{g}\left(\sqrt{\mathbf{G}_{i} \mathbf{G}^{i}}\right)$ and separated by a time lapse $\Delta$, known as the diffusion time, the Equation 2.15 can be solved analytically. The resulting signal amplitude at TE is given by:

$$
\ln \left(\frac{S(T E)}{S(0)}\right)=-\gamma^{2} D \delta^{2}(\Delta-\delta / 3) g^{2}
$$

The resulting Equation 2.17 is called the Stejskal-Tanner equation [192]. This shows the main effect produced by the use of the diffusion gradients: if water molecules diffuse then the second diffusion gradient cannot rephase completely the spins dephased by the first diffusion gradient. This is considered as an incomplete rephase process. The resulting signal gets attenuated (as shown in Equation 2.17) and it is recorded independently of the gradient shape $\left(\mathbf{G}(t)^{i} \neq \mathbf{G}^{i}\right)$. On the other hand, if the water molecules are static, then the spins are fully rephased and the signal is not affected by the diffusion gradients.

The expression in Equation 2.17: $\gamma^{2} \delta^{2}(\Delta-\delta / 3) g^{2}$, defines the dMRI parameter called b-value (b) [in units of (time)(length) ${ }^{-2}$ ]. This parameter summarises the diffusion strength (or weighting) applied to the signal.

The experiment performed by Stejskal and Tanner can be extended for other MR sequences as shown in the next sections.

STEAM and dSTEAM sequence

A variant of the $\mathrm{SE}$ sequence is the stimulated echo acquisition method (STEAM) sequence [85]. In this sequence, the refocusing RF pulse $\left(\alpha_{2}\right.$ in 
Figure 2.6) is replaced by two extra RF pulses with their phases equal to the phase of the excitation pulse. Instead of reversing the dephasing of spins and making them rephase at echo time when the $\alpha_{2}$-pulse is used (in $\mathrm{SE}$ ), the first refocusing RF pulse (called the storing pulse, $\alpha_{2}$ ) distributes the spins between the transverse plane and the longitudinal plane. That means that a certain fraction (dependent on $\alpha_{2}$ value) of the spins will create a spin-echo (SE) signal. Meanwhile, the remaining fraction will be stored and will experience $T_{1}$ relaxation. The second refocusing $R F$ pulse (called the recalling pulse, $\alpha_{3}$ ) will recover the spins stored in the longitudinal plane and consequently will create a stimulated echo (STE) signal. The time between the storing and recalling pulses is called the mixing time (TM, $\tau_{2}$ in Figure 2.7). The corresponding sequence diagram is shown in Figure 2.7 (obtained from Chapter 5).

Solving the corresponding Bloch equation for this sequence, both signals

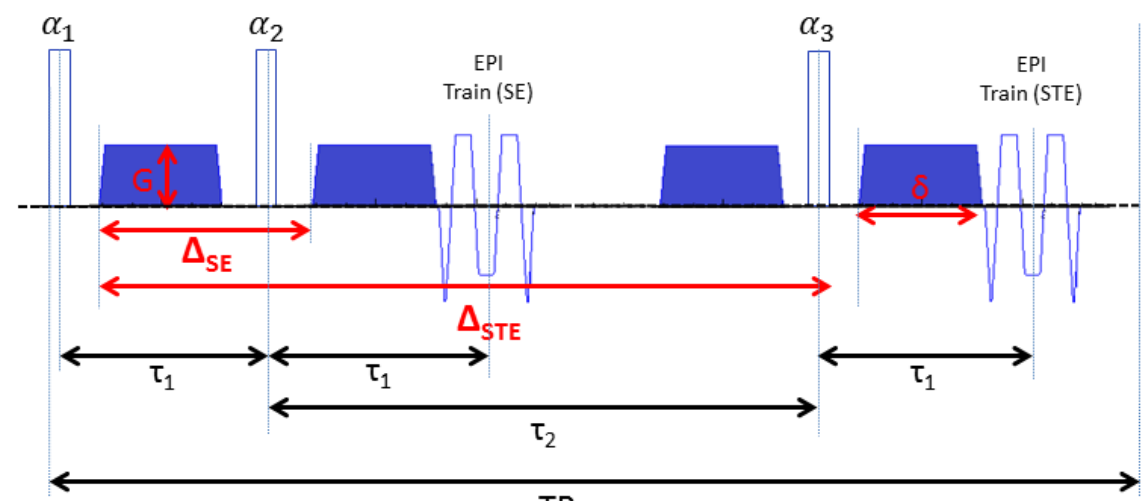

TR

Figure 2.7: dSTEAM sequence: Sequence diagram where the excitation $\left(\alpha_{1}\right)$, storing $\left(\alpha_{2}\right)$ and recalling $\left(\alpha_{3}\right)$ RF pulses are indicated. At time TE $\left(2 \tau_{1}\right)$ is when the spin-echo is formed and encoded by the readout gradient (EPI train). The time between the storing and recalling pulses is known as mixing time (TM). At time TM $+\mathrm{TE}\left(2 \tau_{1}+\tau_{2}\right)$ the stimulated echo (STE) is formed and encoded by another readout gradient (EPI train). Diffusion gradients (in blue) are added to convert the STEAM sequence to a dSTEAM sequence. Those gradients can be added to weight the SE and STE signals by diffusion differently (i.e. at different diffusion times, $\Delta_{\mathrm{SE}}$ and $\Delta_{\mathrm{STE}}$ respectively). The other parameters such as diffusion gradient time ( $\delta$ ) and diffusion gradient amplitude (G) are indicated. 
(the SE and STE) are defined as follows:

$$
\mathrm{SE}: \quad S_{S E}\left(\alpha_{i}, T E, T_{2}\right)=M_{z} \sin \left(\alpha_{1}\right) \sin \left(\frac{\alpha_{2}}{2}\right)^{2} \exp \left(\frac{-T E}{T_{2}}\right)
$$

STE: $\quad S_{S T E}\left(\alpha_{i}, T E, T M, T_{1}, T_{2}\right)=M_{z} \cdot 0.5\left(\prod_{i=1}^{3} \sin \left(\alpha_{i}\right)\right) \exp \left(\frac{-T E}{T_{2}}+\frac{-T M}{T_{1}}\right)$

Where $\mathrm{M}^{\mathrm{z}}$ is the total available bulk magnetisation in the sequence (see Chapter 5 for a deeper description of this parameter in function of the timing parameters). It should be noted that the STEAM sequence can create three more SE's signals (the second, third and fourth spin-echo) after the STE, which are a consequence of the free induction decay (FID) signal generated by the storing and recalling pulses. However, the focus of this thesis is on the primary SE and, mostly, the STE signals. It should also be noted that the third diffusion gradient, just prior to the recalling pulse, is strictly unnecessary for the diffusion-weighting of the primary SE and STE signals. However, it assist in the (partial) crushing of other (undesired) SE signals.

This sequence, in comparison to PGSE, possesses some advantages and disadvantages. The resulting SE and STE signals are intrinsically lower (in intensity, at least half) in comparison to the SE signal obtained from SE, and the required amount of time for data acquisition (TR comparison) is longer (and dependent of the duration of TM). On the other hand, STEAM allows to obtain two signals weighted differently. As shown in Equations 2.18 and 2.19 , the SE and STE signals are equally weighted by $\mathrm{T}_{2}$. However, the STE is also weighted by $\mathrm{T}_{1}$. Besides that, the SE and STE signals can also be weighted by diffusion differently. As shown in Figure 2.7, at similar diffusion gradient amplitude and duration, the corresponding diffusion times $\left(\Delta_{\mathrm{SE}}\right.$ and $\left.\Delta_{\mathrm{STE}}\right)$ are different. Although they can be calculated similarly as in the dPGSE case (by solving Equation 2.15), the corresponding b-values for SE and STE signals in STEAM sequence are different:

$$
\begin{array}{r}
b_{S E}: \gamma^{2} G^{2} \delta^{2}\left(\Delta_{S E}-\delta / 3\right) \\
\mathrm{dSE}: \quad M_{x y}\left(\alpha_{i}, \tau_{1}, T_{2}\right)=S_{S E}\left(\alpha_{i}, T E, T_{2}\right) \exp \left(-b_{S E} D\right) \\
b_{S T E}: \gamma^{2} G^{2} \delta^{2}\left(\left(\Delta_{S E}+T M\right)-\delta / 3\right) \\
\mathrm{dSTE}: \quad M_{x y}\left(\alpha_{i}, \tau_{1}, \tau_{2}, T_{1}, T_{2}\right)=S_{S T E}\left(\alpha_{i}, T E, T M, T_{1}, T_{2}\right) \exp \left(-b_{S T E} D\right)
\end{array}
$$


As shown in the following chapters, this intrinsic property becomes relevant for neuroanatomical studies.

SSFP and dSSFP sequence

In comparison to the aforementioned sequences, the steady-state free precession (SSFP) is a GRE-based sequence (section 2.1.2). This means that only one RF pulse is used for excitation and the echoes are created by gradient dephasing-rephasing (shown in Figure 2.3). This sequence was firstly introduced by Gyngell et al. in 1988 [98] and further developed by Buxton et al. in 1993 [41]. Another feature of this sequence is that the signal is not purely gradient-echo, SE or STE, but instead the resulting signal is a combination of different echoes acquired in different time-series. The corresponding sequence diagram is shown in Figure 2.8 (obtained from Chapter 4).

Its modification to acquire diffusion-weighted MR data using this se-

dSSFP (CE-FAST)

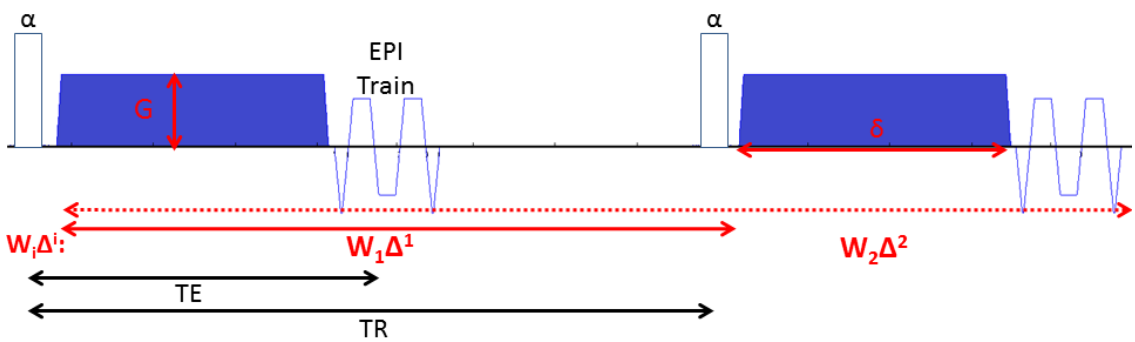

Figure 2.8: dSSFP sequence: Sequence diagram where the excitation $\left(\alpha_{1}\right)$ RF pulse is indicated. At time TE, the echo is formed and encoded by the readout gradient (EPI train). Diffusion gradients (in blue) are added to convert the SSFP sequence to a dSSFP sequence. In comparison to dPGSE, the total diffusion-weighting in the gradient-echo signal from SSFP is equal to a linear combination with specific weights $\left(\mathrm{w}_{\mathrm{i}}\right)$ of diffusion times $\left(\Delta_{\mathrm{i}}\right)$ applied at different time-series $\left(\mathrm{w}_{\mathrm{i}} \Delta^{\mathrm{i}}\right)$. The other parameters as diffusion gradient time $(\delta)$ and diffusion gradient amplitude $(\mathrm{G})$ are indicated.

quence was introduced recently [41, 143]. Due to its complex signal mixing behaviour, the corresponding b-value is not obtained straightforward as for dPGSE or dSTEAM. As shown in [41], the resulting diffusion-weighted 
signal is defined as follows:

$$
\begin{array}{r}
S_{d S S F P}: \frac{-M_{0}\left(1-E_{1}\right) E_{2} A_{2}^{-2 / 3}\left(F_{1}-E_{2} A_{1} A_{2}^{2 / 3}\right) \sin (\alpha)}{r-F_{1} s} \text { where } \\
F_{1}: K-\sqrt{K^{2}-A_{2}^{2}}, K: \frac{1-E_{1} A_{1} \cos (\alpha)-E_{2}^{2} A_{1}^{2} A_{2}^{-2 / 3}\left(E_{1} A_{1}-\cos (\alpha)\right.}{E_{2} A_{1} A_{2}^{-4 / 3}(1+\cos (\alpha))\left(1-E_{1} A_{1}\right)} \\
r: 1-E_{1} \cos (\alpha)+E_{2}^{2} A_{1} A_{2}^{1 / 3}\left(\cos (\alpha)-E_{1}\right) \\
s: E_{2} A_{1} A_{2}^{-4 / 3}\left(1-E_{1} \cos (\alpha)\right)+E_{2} A_{2}^{-1 / 3}(\cos \alpha-1) \\
A_{1}: \exp (-b D), \quad A_{2}: \exp (-\beta D), \quad b:(\gamma G \delta)^{2} T R, \quad \beta:(\gamma G \delta)^{2} \delta, \\
E_{1}: \exp \left(-T R / T_{1}\right) \quad \text { and } \quad E_{2}: \exp \left(-T R / T_{2}\right)
\end{array}
$$

This dMRI sequence possesses advantages in comparison to PGSE-based sequences (e.g. dPGSE and dSTEAM) as presented in [143, 144, 147, 84]. Some of those are high signal-to-noise ratio efficiency (SNR-eff), which is related to the amount of signal acquired in a period of time; and very fast acquisition time. A better description of this sequence is developed in Chapter 4, and conceptually compared to STEAM in Chapter 3.

\section{Diffusion acquisition and analysis}

As shown in the previous sections, the use of Equation 2.17 can be useful to study systems with isotropic self-diffusivity using any dMRI sequence. However, it can be extended to study anisotropic media as well. This is achieved by applying a total diffusion gradient defined as a linear combination of diffusion gradients along all the axes $\left(\mathbf{G}^{i}=g_{i} \mathbf{e}^{i}\right)$. The corresponding vector magnitude results in the b-value, Equation 2.17) [191]. By rearranging Equation 2.15 for anisotropic diffusion and for a given diffusion gradient vector $\left(\mathbf{G}^{\mathrm{i}}\right)$, the resulting signal decay is given by:

$$
\ln \left(\frac{S(2 \tau)}{S(0)}\right)=-\gamma^{2}\left(g_{i} \delta_{j}^{i} g^{j}\right) \delta^{2}(\Delta-\delta / 3)\left(\mathbf{e}_{i} \mathbf{D}^{i}{ }_{j} \mathbf{e}^{j}\right)
$$

where $\delta_{j}^{i}$ is the Kronecker delta. One example of anisotropic media is living tissue. At body temperature, the estimated water self-diffusivity in human body, at free and isotropic conditions, is around $3.0 \times 10^{-3} \mathrm{~mm}^{2} / \mathrm{s}$. However, this value changes because the water molecules are hindered or restricted (or contained) in several biological compartments: the intra-cellular space 
(inside the cells) and in the extracellular space (in between the cells) [127]. Those spaces have a particular geometry (e.g. cell shape) or distribution (e.g. cell arrangement) depending on the organ or organism. The most commonly studied specimen with this technique, and the object of interest in this thesis, is the brain.

The brain is part of the central nervous system (CNS), protected by the skull and a protein-rich solution called cerebral-spinal fluid (CSF). The brain contains several types of cells: the neurons (representing $10 \%$ of the total number of cells) and the glial cells (representing the remaining 90\% of cells) [218] $]^{6}$. The neuron is responsible for brain signal transmission and brain connectivity. It consists of a cell body (soma) with narrow processes (dendrites) and, in most of the neurons, a single long process called axon (Figure 2.9B), which can branch heavily. The neurons are distributed in the brain in such a way that it creates a structural division: the white matter (WM) and the grey matter (GM). The WM is the area where primarily remotely projecting axons are extended and they connect between different areas of the GM. The majority of the axons in the WM are aligned to create well-organised tracts, but these can also cross each other [188]. Most of the corresponding somas lie in the GM in specific locations. Depending on the density and distribution of those, the GM can be subdivided in structures called layers [38, 217].

Apart from cells, another important element to consider in the cerebral architecture is the vasculature. It has been shown (i.e. for rats see reference [120], and humans see reference [141]) that arterial blood vessels penetrate straight through the cortex and terminate along the way or even beyond it, like in the subcortical white matter. On the other hand, the capillary networks in the cortex are three times more dense than those in the white matter.

Due to the wide variety of cell types and structures in the brain, it is necessary therefore to establish several assumptions. Those need to be made to model how the water molecules will diffuse in this organ:

1. Due to its chemical composition, it can be assumed that the CSF is nearly a Newtonian fluid and it can be approximated as water [32].

2. Since the living brain is thermally regulated at $37^{\circ} \mathrm{C}$ (or $310 \mathrm{~K}$ ), the

\footnotetext{
${ }^{6}$ However, this distribution of cells in the human brain is still under discussion, for example in the review of [103] reports that just in few regions of the brain this proportion applies. In other areas, like the grey matter, the ratio between neurons and glial cells is almost $1: 2$
} 


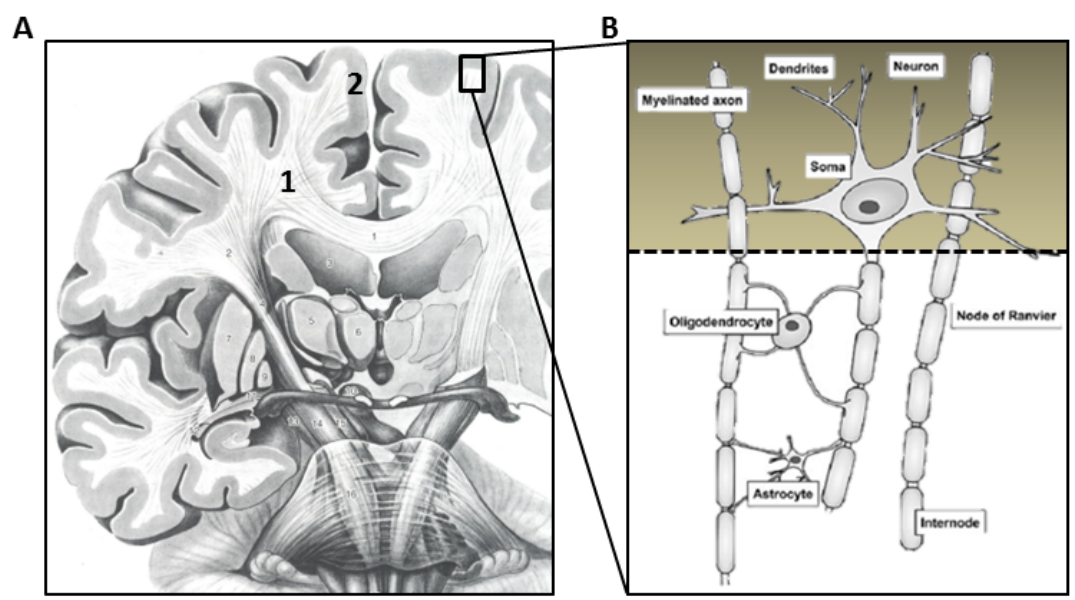

Figure 2.9: Brain anatomy: (A) Coronal brain slice where main structures like white matter (1) and grey matter (2) are indicated. (B) A simplified view of the zoomed area in (A) which describes the cellular distribution of the brain cells in the grey matter area (gray zone) and white matter area (white zone)

self-diffusivity of the water molecules is almost constant $\left(\sim 3 \times 10^{-3}\right.$ $\left.\mathrm{mm}^{2} / \mathrm{s}\right)$.

3. Taking both points above, the differences in the self-diffusivity values when estimated can be explained directly due to the geometry of the cells and the cell density in the GM and WM structures.

4. For WM, self-diffusivity is considered anisotropic and dependent on the axonal packing. This anisotropy is considered as a combination of the restricted diffusivity in the axons and the hindered space in the extracellular space [127]. Depending on the time scale (Figure 2.5), boundaries and approximations must be considered to estimate this parameter: by approximating the axons as cylinders with a specific radius (as defined in references [44, 56, 213]) as similarly found in literature (e.g. see reference [134]) or infinitely narrow (i.e. sticks) (as in reference [26]).

5. In GM, self-diffusivity can be assumed to be isotropic. However, it is expected that at high resolutions the self-diffusivity becomes anisotropic due to axonal bundles (close to the WM/GM boundary) and pyramidal 
apical dendrites. Moreover, this can be also influenced by the density and/or distribution of blood vessels. This anisotropic behaviour is shown in Chapter 3.

In the context of dMRI applied to brain studies, fully characterising $\mathbf{D}^{i}{ }_{j}$ can bring insights about how the fibre bundles are oriented in WM. To fully estimate $\mathbf{D}^{i}{ }_{j}$ by using Equation 2.23, it is necessary to acquire at least 6 noncollinear and evenly spaced b-vectors at a constant high b-value (typically $1000 \mathrm{~s} / \mathrm{mm}^{2}$ ), plus a near-zero low b-value volume (or volumes) as reference. This acquisition scheme and the estimation of $\mathbf{D}^{i}{ }_{j}$ is called Diffusion Tensor Imaging (DTI) [18]. After estimating $\mathbf{D}^{i}$, the predominant diffusion orientations and corresponding magnitudes can be obtained by eigendecomposing it (as shown in Equation 2.13). Other derived parameters can be calculated like the fractional anisotropy (FA) and mean diffusivity (MD). The FA quantifies the normalised variance between the eigenvalues. An FA of 0 represents isotropic diffusion and an FA of 1 represents perfectly uni-directional anisotropic diffusion. The MD, on the other hand, quantifies the total amount of diffusion calculated as the average of the eigenvalues. It has been shown that these parameters can be used as bio-markers to characterise brain structures, developmental structural changes (e.g. in reference [17]) in healthy brains, as well as to detect anomalies in diseased brains (e.g. in reference $[96,66]$ ) or to study the structure of ex vivo brain samples (e.g. in reference [97] - or in Chapters 3 and 6).

It can be expected that under certain conditions (larger b-value or higher b-vector sampling) the signal attenuation in the brain and hence the estimated $\mathbf{D}^{i}{ }_{j}$ does not follow the approximation shown in Equation $2.23^{7}$. Moreover, specificity gets reduced in certain brain structures because the averaged self-diffusivity in several biological structural configurations (i.e. axon packing/distribution) can be approximately the same due to spatial resolution [117]. This can be counteracted by increasing the total amount of data using higher $b$-values and/or higher sampling of b-vectors. In this case, the use of DTI becomes counterproductive because the model becomes unsuitable for high b-values and a high amount of data (e.g. see reference [9]). For this reason, during the last decades, more complex models were introduced. As summarised in the work of Jelescu et al. [108], some of those models characterise empirically (or physically) the diffusion signal behaviour without any underlying prior information about the tissue in consideration. Even if the results can be considered accurate, they

\footnotetext{
${ }^{7}$ In other words, the self-diffusivity is not Gaussian.
} 
A

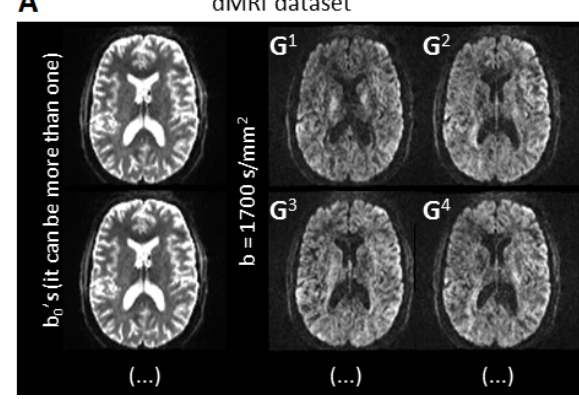

B

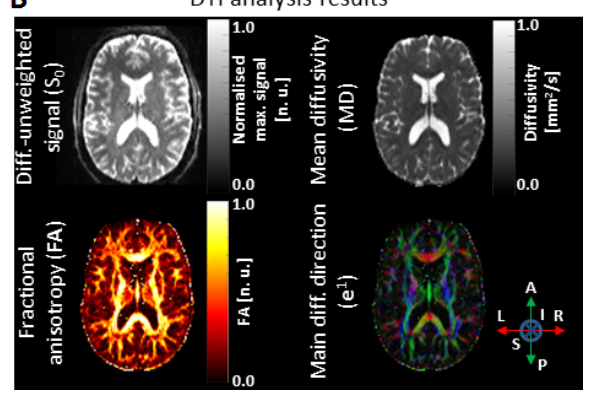

Figure 2.10: DTI acquisition and analysis: (A) A dMRI dataset with the two different types of volumes, both needed for analysis: (at least) one non-diffusion-weighted image (or $\mathrm{b}_{0}$ ) and (at least) one diffusion-weighted image. It is clear how the use of different b-vectors $\left(\mathbf{G}^{\mathrm{i}}\right)$ shows different signal contrast, which is proportional of the water self-diffusivity along that specific direction. For DTI analysis, at least 6 non-collinear dMRI images are required. (B) Some DTI results parameters like the unweighted-diffusion signal (expected MR signal without diffusion component, $\mathrm{S}_{0}$ ), fractional anisotropy (normalised variance between the eigenvalues obtained from the decomposed diffusion tensor, FA), mean diffusivity (average of the eigenvalues, MD) and the diffusion main orientation (RGB colour encoded direction of the first eigenvector) are shown.

don't provide biological information (or requires a cautious interpretation) from the analysed signal. Those models are referred as physical (or signalbased) models. On the other hand, other models take into consideration the tissue (micro-)structure through compartments. The total signal is then a sum of compartments, in which each compartment represents the signal coming from a biological structure (e.g. extra/intra-cellular volumes, axons and cells)[161]. Fitting the diffusion data to those models can provide quantitative parameters with greater specificity and better biological interpretation. Those models are referred as bio-physical (or compartment) models. In the following list, a (sub) group of dMRI signal models (physical and bio-physical) with their corresponding definition, minimum data quantity and approximations are shown. ${ }^{8}$

- Physical models:

- Diffusion Kurtosis Imaging (DKI) [111]: It is understood as the correction or perturbation of the Gaussian distribution of the

\footnotetext{
${ }^{8}$ Only the models used along this thesis are introduced.
} 
water self-diffusivity. It is characterised by the kurtosis (K) of this distribution. As it is defined in literature (in reference [111], for example), this model requires at least 21 (diffusion-weighted) volumes at $b=1000 \mathrm{~s} / \mathrm{mm}^{2}(6)$ and $b=2000 \mathrm{~s} / \mathrm{mm}^{2}$ (15). This model keeps the same parameters from DTI (like FA or MD - but corrected) but extra parameters like mean kurtosis (MK) can be used for studies [193].

- Bio-physical models: In a general approximation, three compartments are defined: the extra-cellular space (ECS), the intra-axonal space (IAS) and the intra-cellular/free space (FS). Depending on the assumptions of models used on each of them, the following models are defined.

- Ball \& Stick Model [26]: All the compartments, except the axons, diffuses freely. The axons, on the other hand, are defined as parallel zero-radius cylinders (i.e. sticks). This model is used mostly for estimating orientation profiles. In the model can be assumed from one to three sticks. It is not an established requirement about a minimum dataset, however it must be equal or higher than the dataset used for DTI (i.e. 6 volumes at 1000 $\mathrm{s} / \mathrm{mm}^{2}$ ).

- Neurite orientation dispersion and density imaging (NODDI) [237]: As similar to the Ball \& Stick model, the FS is modelled as a free diffusivity compartment and the IAS is modelled as sticks. However, those are not arranged in parallel but they follow an orientation distribution defined by the Watson distribution [140, 236]. The ECS, on the other hand, is modelled by the anisotropic DTI model. By reported in literature (in reference [237]), $\mathrm{a} \mathrm{b}=1000 \mathrm{~s} / \mathrm{mm}^{2}$ with 30 directions and $\mathrm{b}=2000 \mathrm{~s} / \mathrm{mm}^{2}$ with 60 directions.

- Composite hindered and restricted model of diffusion (CHARMED) [8]: In this model, two spaces are modelled. The IAS is defined by impermeable parallel cylinders with a gamma distribution of radii. The ECS, on the other hand, is defined by an anisotropic DTI model. Years later, the FS is added [15]. By reported in literature (in reference [8]), at least $10 \mathrm{~b}$-values (with maximum of $b=10000 \mathrm{~s} / \mathrm{mm}^{2}$ ) with $20-30$ directions per b-value are re- 
quired. However, a recent study [61] showed a reduced acquisition scheme that can be used in this model.

In a general perspective, all dMRI models return the (single or multiple) main self-diffusion direction(s). In brain studies, those directions can be combined to infer pathways that cross the brain, resulting in connections between areas. The process to obtain this estimated pathway is often called fibre tracking or fibre tractography [20]. This technique in itself is challenging and is highly dependent on its inputs, approximations and priors [21]. The resulting tracts, even if they are mathematically consistent, do not necessarily represent biological connections. The review in reference [113] presents this technique in detail.

\subsubsection{Biological properties for in vivo and ex vivo human brains}

The study of water self-diffusion using dMRI in the in vivo brain presents several challenges and constraints which could result in a lower specificity of the estimated parameters [116, 117]. This is mainly because the spatial (and diffusion-time) resolution is lower than the effect being studied (e.g. see references $[117,159])$. For that reason, the use of ex vivo whole-brain specimens (e.g. in reference [84]), slices or/and sections (e.g. in references $[59,69])$ have become a promising research field since these can circumvent the spatial and measurement time limitations as shown in Chapter 3 and 4.

However, water diffusion in ex vivo brain specimens has discrepancies in comparison to that in in vivo brains because the biological properties of both differ dramatically. The temperature is reduced from body temperature $\left(37^{\circ} \mathrm{C}\right)$ to room temperature $\left(20^{\circ} \mathrm{C}\right)$, to even close to the freezing point $\left(3\right.$ to $\left.4^{\circ} \mathrm{C}\right)$ for preservation. This difference reduces the water self-diffusivity value by approximately $20 \%$ [104]. Secondly, but also related with the example above, the thermal self-regulation system is not present in ex vivo specimens, therefore the temperature of the specimen can fluctuate during dMRI acquisitions, which would result in an unstable (or biased) estimation of the self-diffusivity [219]. Another example is that the chemical used to fixate and preserve the specimen modifies it structurally. Some of those effects are the reduction of the extra-cellular space [146] and loss of elasticity of the membrane, and a reduction of the apparent exchange rate $[123,184,185]$. Furthermore, the autolysis pro- 
cess when the brain ceases its function can reduce the amount and density of cells. All these changes (and more, as shown in references [184, 185]) are translated in some diffusion-related results, like the mean diffusivity which gets reduced from the in vivo values to almost half in ex vivo situations (as shown in Chapter 3). Therefore, the biological interpretation of the obtained dMRI results requires a cautious and comprehensive understanding of water self-diffusion, time and spatial scales.

\subsection{Structure of this thesis}

This thesis deals with the study of human brain structure (at the macro-, meso- and microscale), from the MRI perspective, especially from diffusion MRI. Throughout it leverages the advantages, and finds solutions to the challenges, of ultra-high field strenght MRI at 7T and 9.4 T. It approaches the study of brain structure, both ex-vivo and in-vivo, with specific advances in MRI pulse sequences and image analysis methods that optimally use the data provided by these pulse sequences. The work presented in this thesis establishes, studies, analyses and solves the following questions:

- How can the dSTEAM and dSSFP sequences be used for high resolution ex vivo dMRI studies of the intact human brain at ultra-high field strengths?

- How can the dSTEAM sequence be used for highly efficient quantitative multi-contrast and dMRI in vivo studies of the human brain at ultra-high field strengths?

- Can the advantages of the sequence and the corresponding results be used for a better understanding of dMRI applied on ex vivo and in vivo brains?

This thesis then is structured in the following chapters, which aim to answer the questions established above:

- Chapter 3 shows how the dSTEAM sequence can be optimised with parallel RF transmission techniques and tailored analysis for high resolution ex vivo dMRI studies at 9.4 T. Together with Chapter 4, it targets question 1 above. 
- Chapter 4, a short intermediate chapter, takes the pulse sequence focus away from dSTEAM, and shows how the dSSFP sequence can be optimised with parallel RF transmission techniques for high resolution ex vivo dMRI studies at $9.4 \mathrm{~T}$.

- Chapter 5 performs an in-depth signal analysis of the STEAM sequence, particularly on the steady-state effects on both SE and STE signals in short-TR circumstances. It discusses both optimisation of measurement protocols to avoid unexpected physics interaction in the SE and STE signals, and non-intuitive contrast mechanisms that can be utilised.

- Chapter 6 shifts the target from ex vivo human brain acquisitions to in vivo acquisitions, and from high resolution studies to quantitative multi-contrast studies as a potential strong point of STEAM at UHF. It presents the MESMERISED sequence, which achieves super-accelerated 7 T STEAM imaging by combining echo-shifting and simultaneous multi-slice acceleration. It evaluates MESMERISED as a technique that can efficiently probe combined $T_{1}, T_{2}$ and diffusion contrast, fast multi-contrast mapping and characterisation of multi-component relaxometry, diffusion, and exchange.

- Chapter 7 gives a general overview of the thesis and discusses the outcomes in a broader perspective, while giving an outlook on possible future developments emanating from it.

- Finally, Chapter 8 briefly summarises the knowledge utilisation and valorisation aspects of the research presented in this thesis. 



\section{$\mathrm{k}_{\mathrm{T}}$-dSTEAM: Ultra-high resolution and multi-shell diffusion MRI of intact ex vivo human brains at 9.4}

\footnotetext{
${ }^{1}$ From the published article: "Fritz et al. Ultra-high resolution and multi-shell diffusion MRI of intact ex vivo human brains using $k_{T}$-dSTEAM at 9.4T, NeuroImage, Vol. 202, 2019. DOI: https://doi.org/10.1016/j.neuroimage.2019.116087" and from the accepted abstracts: "Fritz et al. KtextsubscriptT-dSTEAM: high resolution diffusion-weighted imaging of the ex vivo human brain using $B_{1}+$ homogenized STEAM at $9.4 T$. ISMRM 26th Annual Meeting and Exhibition, Honolulu, 2017" (accepted as Oral Presentation and awarded with Magna cum Laude), "Fritz et al. Ultra-high resolution multi-shell dMRI and tractography of the ex vivo human brain using ktextsubscriptT-dSTEAM at 9.4T. ISMRM 27th Annual Meeting and Exhibition, Paris, 2018" (accepted as Oral Presentation), "Fritz et al. ktextsubscriptT-STEAM: ktextsubscriptT-points 3D STEAM at 9.4T for high resolution whole brain $T_{1}$ and $T_{2}$ weighted MRI ex vivo. ISMRM Benelux Chapter 9th Annual Meeting and Exhibition, Tilburg, 2017" (accepted as Oral Presentation) and "Fritz et al. High resolution diffusion MRI and tractography of post mortem human brains using $k_{T-}$-STEAM at 9.4T. OHBM 23rd Annual Meeting, Vancouver, 2017" (accepted as Oral Presentation).
} 


\section{Abstract}

Diffusion MRI (dMRI) in ex vivo human brain specimens is an important research tool for neuroanatomical investigations and the validation of dMRI techniques. Many ex vivo dMRI applications have benefited from very high dMRI resolutions achievable on small-bore preclinical or animal MRI scanners for small tissue samples. However, the investigation of entire human brains post mortem provides the important context of entire white matter (WM) network systems and entire grey matter (GM) areas connected through these systems. The investigation of intact ex vivo human brains in large bore systems creates challenges due to the limited gradient performance and transmit radiofrequency $\left(\mathrm{B}_{1}{ }^{+}\right)$inhomogeneities, specially at ultra-high field (UHF, $7 \mathrm{~T}$ and higher). To overcome these issues, it is necessary to tailor ex vivo diffusion-weighted sequences specifically for high resolution and high diffusion-weighting. Here, we present $\mathrm{k}_{\mathrm{T}}-\mathrm{dSTEAM}$, which achieves $\mathrm{B}_{1}{ }^{+}$homogenisation across whole human brain specimens using parallel transmit (pTx) on a 9.4 T MR system. We use $\mathrm{k}_{\mathrm{T}}$-dSTEAM to obtain multi-shell high b-value and high resolution diffusion-weighted data in ex vivo whole human brains. Isotropic whole-brain data can be acquired at high b-value $\left(6000-8000 \mathrm{~s} / \mathrm{mm}^{2}\right)$ at high resolution $(1000 \mu \mathrm{m})$ and at moderate $b$-value $\left(3000 \mathrm{~s} / \mathrm{mm}^{2}\right)$ at ultra-high isotropic resolution $(400 \mu \mathrm{m})$. As an illustration of the advantages of the ultra-high resolution, tractography across the WM/GM border shows less of the unwanted gyral crown bias, and more high-curvature paths connecting the sulcal wall than at lower resolution. The $\mathrm{k}_{\mathrm{T}}$-dSTEAM also allows for acquisition of $\mathrm{T}_{1}$ and $\mathrm{T}_{2}$ weighted images suitable for estimating quantitative $\mathrm{T}_{1}$ and $\mathrm{T}_{2}$ maps. Finally, multi-shell analysis of $\mathrm{k}_{\mathrm{T}}$-dSTEAM data at variable mixing time (TM) is shown as an approach for ex vivo data analysis which is adapted to the strengths of STEAM diffusion-weighting. Here, we use this gain for multi-orientation modelling and crossing-fibre tractography. We show that multi-shell data allows superior multiple orientation tractography of known crossing fibre structures in the brain stem.

\subsection{Introduction}

Diffusion MRI (dMRI) in ex vivo human brain specimens is an important research tool for neuroanatomical investigations and for the validation of dMRI techniques. For instance, ex vivo dMRI studies have focused on val- 
idation of white matter orientation estimates [129, 178], microstructure models [12, 129, 150] and tractography [173, 179], as well as the atlasing and mapping of human subcortical structures $[2,67]$ and the delineation of grey matter layers [3, 22, 121, 131]. All ex vivo applications benefit from high (1000 $\mu \mathrm{m}$ isotropic and far below) dMRI resolution, which is difficult to achieve primarily because of two effects. First, since post mortem tissue possesses strongly reduced diffusivity compared to in vivo tissue, the required b-values for diffusion analysis must be proportionally higher, often at least doubled [59, 147]. Second, the strongly reduced $\mathrm{T}_{2}$ of fixed tissue [163] means $\mathrm{T}_{2}$-weighted signal is lower and signal-to-noise ratio (SNR) is substantially reduced, especially for relatively long echo times (TE's). Ex vivo dMRI using the pulsed gradient spin-echo (PGSE) sequence, which is the most common dMRI pulse sequence, is especially challenged due to the strong interaction of both these effects. Using high b-values with PGSE to compensate the lower diffusivity inherently implies longer TE's, and therefore strong signal decay.

Nonetheless, high quality ex vivo dMRI results can be achieved with PGSE on small-bore preclinical or animal MRI systems (e.g. [3, 173, 179, 42, 79, $92,151,210])$, where post mortem tissue dMRI has so far been predominantly performed. This is due to the superior gradient performance of small-bore preclinical systems, often exceeding five times that of largebore systems, allowing short TE/high-b PGSE acquisitions. In addition, ex vivo imaging often involves scanning post mortem tissue over long scan sessions (many hours to a couple of days) yielding ultra-high resolution datasets far below the millimetre scale while maintaining a sufficiently high SNR for the acquired images. However, the use of small-bore systems is often limited to small human tissue samples (often less than about $20 \mathrm{x}$ $20 \times 20 \mathrm{~mm}^{2}$, and generally smaller than about $50 \times 50 \times 50 \mathrm{~mm}^{2}$ ), severely restricting the size of the brain region examined.

Here we consider the investigation of entire formalin fixed human brains or hemispheres post mortem to provide the important context of entire white matter (WM) network systems and entire grey matter areas connected through these systems. Since the acquisition of ex vivo dMRI for intact samples of this size necessarily has to take place in large bore systems, this re-introduces the challenge of limited gradient performance compared to small bore systems, and the relative inefficiency of PGSE. Therefore, it is necessary to find and optimise other diffusion-weighted sequences for high resolution and high diffusion-weighting for this specific case. Earlier work by [147] and [84] has shown that the use of diffusion-weighted SteadyState Free Precession (DW-SSFP) for dMRI acquisition of the whole-brain 
ex vivo at $3 \mathrm{~T}$ and $7 \mathrm{~T}$ achieves much better SNR efficiency than PGSE. Additionally, the use of ultra-high field (UHF) strength of $7 \mathrm{~T}$ with DWSSFP was shown to further increase SNR efficiency and contrast-to-noise ratio (CNR) for whole-brain ex vivo dMRI acquisitions, in comparison to $3 \mathrm{~T}$. However, a disadvantage of the DW-SSFP sequence is that the signal has a complex dependence on diffusion-weighting, $\mathrm{T}_{1}, \mathrm{~T}_{2}$, and flip angle $[41,144]$, making its analysis difficult, especially in the case of multi-shell acquisitions.

Therefore, we consider here the diffusion-weighted STimulated Echo Acquisition Mode (dSTEAM) sequence [145]. The dSTEAM pulse sequence can create a large part of its diffusion contrast during the slower $\mathrm{T}_{1}$ decay (i.e. during the mixing time while the spins are stored in the longitudinal axis), rather than during the faster $\mathrm{T}_{2}$ decay. This is advantageous because it overcomes the challenges already mentioned for post mortem whole-brain imaging: shortened $\mathrm{T}_{2}$ and required high $\mathrm{b}$-values. In addition, since $\mathrm{T}_{1}$ increases and $\mathrm{T}_{2}$ decreases with increasing field strength, this advantage of dSTEAM is amplified at UHF. Compared to a PGSE acquisition, the main disadvantage of dSTEAM is that it uses only half of the total signal available for the stimulated echo (STE) signal. An effective use of dSTEAM for whole-brain post mortem dMRI, then, depends on compensating the need to work with half of the signal with its beneficial effects in the case of UHF ex vivo imaging with low-performing gradients. One general complication of working with large samples at UHF is that $\mathrm{B}_{1}{ }^{+}$inhomogeneity is enhanced, which negatively affects the contrast and signal homogeneity of the acquired data. For instance, at $9.4 \mathrm{~T}$ the required $\mathrm{RF}$ excitation wavelength is approximately $8 \mathrm{~cm}$ (in brain tissue this wavelength is shorter due to its relative permittivity) which leads to destructive interferences along a whole-brain specimen. In the case of dSTEAM, the combination of three consecutive pulses required to obtain the desired STE signal means that $\mathrm{B}_{1}{ }^{+}$inhomogeneities are exacerbated to about the third power of those in single excitation sequences (Figure 3.1). In gradient recalled echo (GRE) sequences, it has been shown that the $\mathrm{B}_{1}{ }^{+}$inhomogeneity can be compensated by replacing the non-selective rectangular excitation pulse by a composite pulse created using the $\mathrm{k}_{\mathrm{T}^{-}}$ points method [53], applied in the work of Sengupta et al. 2018 [180] for post mortem tissue. Therefore, extending the $\mathrm{k}_{\mathrm{T}}$-points $\mathrm{B}_{1}{ }^{+}$homogenisation method to the more complex three-pulse situation of dSTEAM would potentially create an effective large field-of-view (FoV) dSTEAM imaging method.

In this work, we address the following objectives for large human brain 
sample ex vivo dMRI: first, we achieve $\mathrm{B}_{1}{ }^{+}$homogenisation across whole human brain specimens using parallel transmit (pTx) on a 9.4 T MR system by integrating the $\mathrm{k}_{\mathrm{T}}$-points technique in the three-pulse dSTEAM sequence, creating $\mathrm{k}_{\mathrm{T}}$-dSTEAM. Second, we use the $\mathrm{k}_{\mathrm{T}}$-dSTEAM sequence to obtain multi-shell high b-value diffusion-weighted data $(6000-8000$ $\left.\mathrm{s} / \mathrm{mm}^{2}\right)$ at high resolution $(1000 \mu \mathrm{m})$ and moderate b-value diffusion-weighted data $\left(3000 \mathrm{~s} / \mathrm{mm}^{2}\right)$ at ultra-high isotropic resolution $(400 \mu \mathrm{m})$, as well as $\mathrm{T}_{1}$ and $\mathrm{T}_{2}$ weighted STEAM images suitable for estimating quantitative $\mathrm{T}_{1}$ and $\mathrm{T}_{2}$ maps. Finally, we perform $\mathrm{T}_{1}$-compensated multi-shell diffusion modelling using $\mathrm{k}_{\mathrm{T}}$-dSTEAM data using the estimated quantitative $\mathrm{T}_{1}$ maps. We illustrate how ultra-high resolution ( $400 \mu \mathrm{m}$ isotropic) wholebrain data can benefit tractography at the white matter/grey matter border and how multi-shell multi-orientation analysis can benefit delineation of crossing fibres in deep white matter.

\subsection{Methods}

\subsection{1 $\mathrm{k}_{\mathrm{T}}$-dSTEAM sequence and $\mathrm{B}_{1}{ }^{+}$inhomogeneity}

The STEAM pulse sequence uses three RF pulses: excitation, storing and recalling pulses, which can create several spin-echoes (SE's) and a stimulated echo (STE), and was first introduced by Frahm and colleagues [85]. In general terms, the excitation pulse places the magnetisation into the transverse plane where it undergoes $\mathrm{T}_{2}$ decay. After that, the storing pulse places ('stores') part of the total magnetisation along the longitudinal axis where it is subject to $T_{1}$ decay during the mixing time (TM). The remaining part of the magnetisation forms the primary SE signal. Finally, the recalling pulse places ('recalls') the stored magnetisation to the transverse plane where it once again undergoes $\mathrm{T}_{2}$ decay until the STE is formed. This sequence can actually create three more SE signals (the second, third and fourth spin-echo) after the STE, which are consequence of the free induction decay (FID) signal generated by the storing and recalling pulses [27]. We focus on the first or primary SE signal in the remainder of this work and refer to it simply as 'the SE' hereafter. Whereas the SE undergoes only $\mathrm{T}_{2}$ decay, the STE signal undergoes both $\mathrm{T}_{1}$ and $\mathrm{T}_{2}$ decay, because the signal is obtained from spins stored along the longitudinal axis for the duration of the TM, making the spins relax under a $\mathrm{T}_{1}$ regime during this time (see reference [133] for more detail). Furthermore, both echoes (SE and STE) share the transverse magnetisation created by the excitation pulse and 
therefore split the total excited signal, which becomes evenly distributed between SE and STE when the storing pulse has a flip angle of $\pi / 2 \mathrm{rad}$ $\left(90^{\circ}\right)$. The resulting magnitude signal of the STE after excitation in an on-resonance condition [133] is defined as:

$$
S_{S T E}=\frac{1}{2}\left(\prod_{i=1}^{3} \sin \alpha_{i}\right) \exp \left(\frac{-T M}{T_{1}}\right) \exp \left(\frac{-T E}{T_{2}}\right)
$$

Here $\alpha_{1}, \alpha_{2}$, and $\alpha_{3}\left(\alpha_{i}\right.$ in general) are the flip angles of excitation, storing and recalling pulses, respectively. From Equation 3.1, it is clear that the maximum signal for STE is achieved when all the flip angles are $\pi / 2$ $\operatorname{rad}\left(90^{\circ}\right)$. Due to the three multiplicative flip angle components in the signal, any $\mathrm{B}_{1}{ }^{+}$inhomogeneity will greatly affect the resulting signal as illustrated in Figure 3.1. Here, for illustration, magnetisation (i.e. signal disregarding relaxation decay) for a single pulse sequence, such as GRE (proportional to $\sin (\alpha)$ ) and for a STEAM sequence (proportional to $\sin (\alpha)^{3}$ ) is derived from an actual post mortem human brain flip angle map at $9.4 \mathrm{~T}$. In UHF scanners, high $\mathrm{B}_{1}{ }^{+}$inhomogeneity over large samples causes signal attenuation and even complete signal loss in some areas. The signal loss and drop-out are already well visible in a single pulse sequence (Figure $3.1 \mathrm{~A}$, left), but can be considerably amplified in the three pulse STEAM sequence (Figure 3.1A, right). Plotting the $\sin (\alpha)$ single pulse magnetisation and the $\sin (\alpha)^{3}$ STEAM magnetisation against flip angle $\alpha$ (Figure 3.1B), shows that signal loss is higher everywhere for the STEAM sequence, but particularly magnified for flip angles $\alpha$ which are moderately off-target, such as the approximately $\pi / 4 \mathrm{rad}\left(45^{\circ}\right)$ flip angle at (2). This translates to a severely amplified signal losses in STEAM in moderately under-flipped regions in the sample (e.g. (2) in Figure 3.1A). It also leads to smaller spatial extent of high signal spots (e.g. (1) in 3.1A) and larger extent of low signal or drop-out spots (e.g. (3) in Figure 3.1A). This demonstrates the increased need for achieving $\mathrm{B}_{1}{ }^{+}$homogeneity for whole-brain STEAM imaging at UHF.

To this end, we introduce the $\mathrm{k}_{\mathrm{T}}$-dSTEAM sequence which is capable of acquiring diffusion-weighted images with improved signal homogeneity in the three pulse STEAM sequence, for whole human brain post mortem imaging.

This proposed sequence (Figure 3.2C) employs composite $\mathrm{k}_{\mathrm{T}}$-points $\mathrm{RF}$ pulses, in contrast to the standard dSTEAM (Figure 3.2A) with simple non-selective rectangular pulses. The resulting $\mathrm{B}_{1}{ }^{+}$profile is improved, which is manifested in a greatly homogenised STEAM signal over the specimen (Figure $3.2 \mathrm{C}$, right). For illustration and comparison, merely replacing only the 


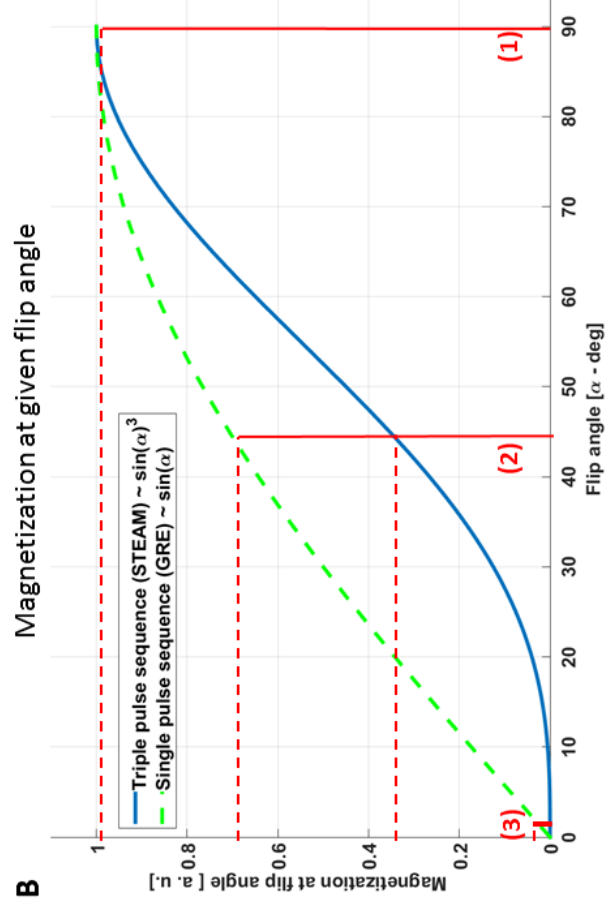

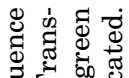

总

屯

궁 च

2.

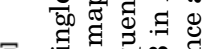

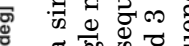

๘

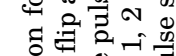

듕

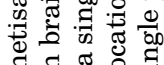

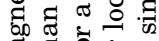

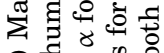

ये

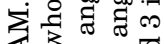

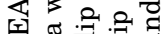

记 है

I

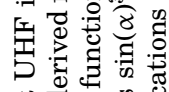

$\infty$

त

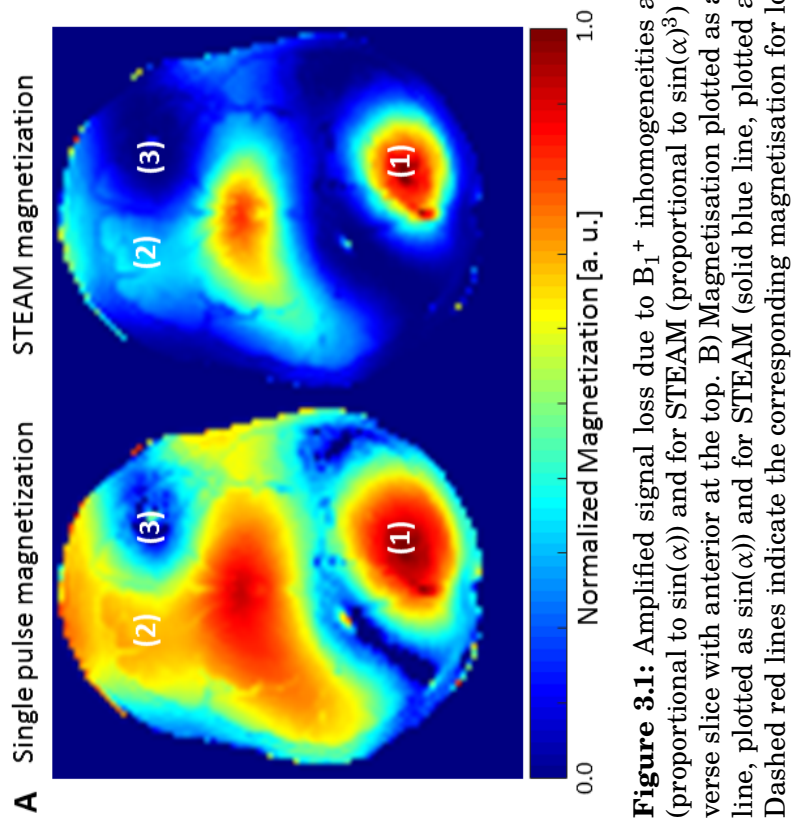


excitation pulse with a composite $\mathrm{k}_{\mathrm{T}}$-points pulse, does not achieve the desired signal homogeneity (Figure 3.2B).

\subsection{2 $\mathrm{k}_{\mathrm{T}}$-dSTEAM signal model}

A full 3D acquisition considerably improves SNR efficiency for whole-brain ex vivo acquisitions [146], but also requires relatively short repetition times (TR's) close to $\mathrm{T}_{1}$ values which means that the signal reaches a steady-state condition. In the case of STEAM, the STE signal possesses an extra component which is dependent on $\mathrm{T}_{1}$, as shown in the references [7, 137]. A proper steady-state is achieved if the signal is completely spoiled before the action of the excitation pulse and the primary SE signal is also completely spoiled before the action of the recalling pulse. If those conditions are fulfilled, the STE signal equation can be adapted to:

$$
S_{S T E}\left(T R, T M, T E, \alpha_{i}\right)=\frac{1}{2}\left(\prod_{i=1}^{3} \sin \alpha_{i}\right)\left(\exp \left(\frac{-T M}{T_{1}}\right)-\exp \left(\frac{-T R}{T_{1}}\right)\right) \exp \left(-\frac{T E}{T_{2}}\right)
$$

The diffusion-weighting of the resulting STE signal requires the use of diffusion gradients before the storing and after the recalling pulses (Figure 3.2). The advantage compared to PGSE is to obtain high diffusionweighting data at short TE's [145], since the diffusion time is extended with TM.

Proper consideration of the $T_{1}$ components in the signal decay, including the TR-TM steady-state term shown in Equation 3.2, would allow for the analysis of diffusion-weighted data from a varying-TM multi-shell dSTEAM acquisition. Since the change in TM implies both a different diffusionweighting and a different $T_{1}$ decay, the combined images can be analysed if the effect of $\mathrm{T}_{1}$ decay is incorporated into the diffusion equation:

$$
\begin{array}{r}
d S_{S T E}=S_{S T E} \exp \left(-(\gamma G \delta)^{2}\left(\Delta_{S E}+T M_{i}-\frac{\delta}{3}\right) D\right) \\
S_{S T E} \exp (-b D) \exp \left((\gamma G \delta)^{2} T M_{i} D\right)
\end{array}
$$

where the b-value, $\mathrm{b}$, is defined as $(\gamma \mathrm{G} \delta)^{2}\left(\Delta_{\mathrm{SE}}-\delta / 3\right)$. If the acquisition scheme for diffusion data only varies TM and all flip angles are considered to be appropriately homogenised and close to $\pi / 2 \mathrm{rad}$, then the Equation 3.3 is simplified to:

$$
d S_{S T E}\left(b, T M_{i}\right)=S_{0}^{*}\left(\exp \left(\frac{-T M_{i}}{T_{1}}\right)-\exp \left(\frac{-T R}{T_{1}}\right)\right) \exp (-b D) \exp \left((\gamma G \delta)^{2} T M_{i} D\right)
$$



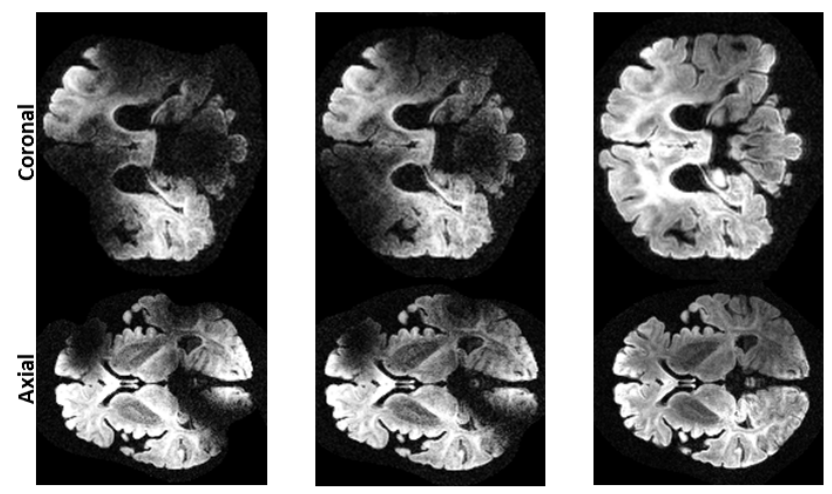

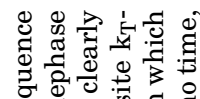

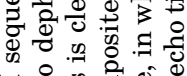

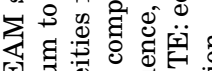

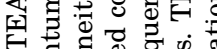

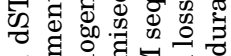

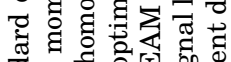

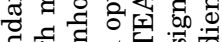
क्षै की य क्ये

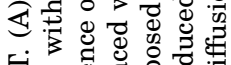
सं क्ष

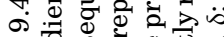
तै कृ की ar 5003

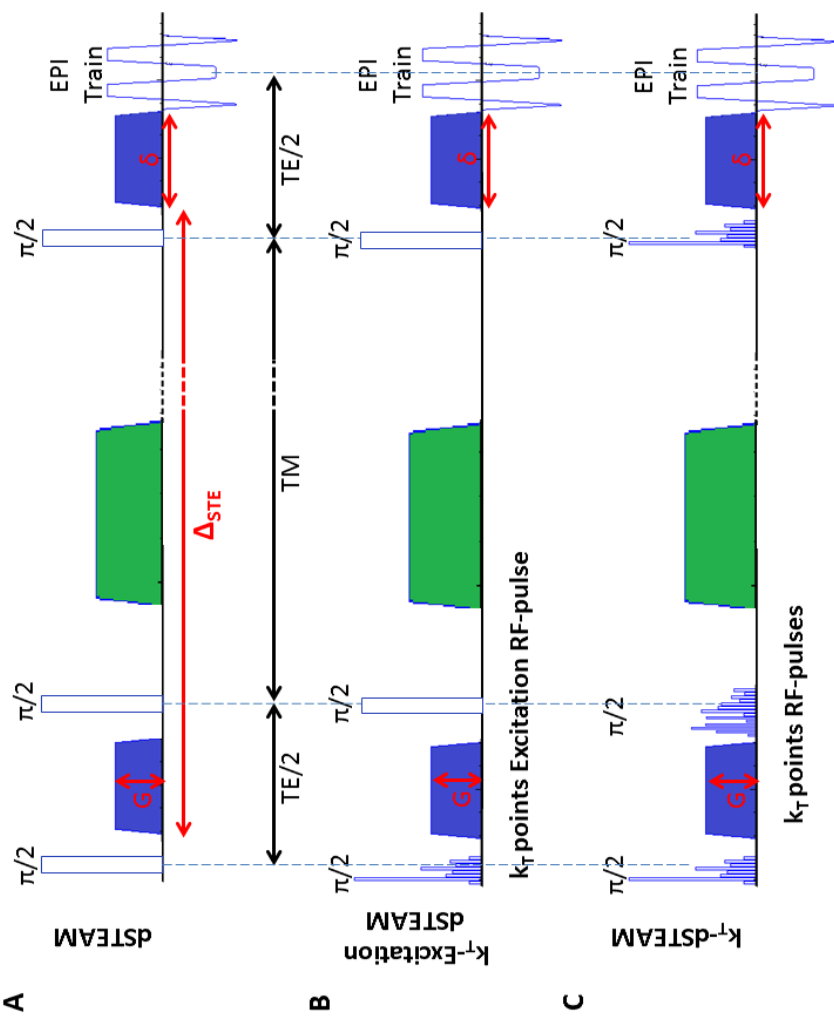

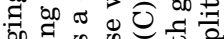

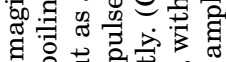

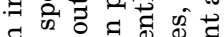

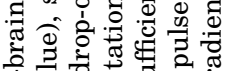

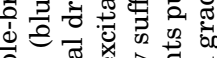

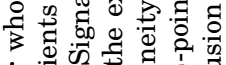

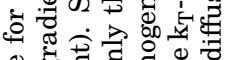
ه

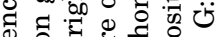

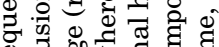
屯 $\sum$ 记

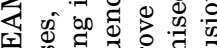

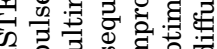

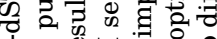
선류 . 원 $>$ के $\sum$ क ব응

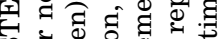

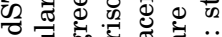
ซ 胥

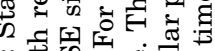

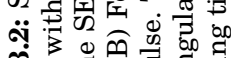
का

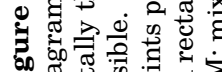

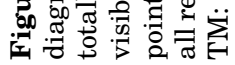


where $\mathrm{S}_{0}{ }^{*}$ represents the $\mathrm{T}_{1}$-unweighted signal (in the limit of $\mathrm{TM}=0$ ). However, this requires $\mathrm{T}_{1}$ to be known, and it must be estimated prior to diffusion analysis, as described below. If all data are acquired at the same TM, the signal equation returns to a form similar to the one used for PGSE:

$$
d S_{S T E}\left(b, T M_{0}\right)=S_{0} \exp (-b D) \exp \left((\gamma G \delta)^{2} T M_{0} D\right)
$$

\subsubsection{Ex vivo specimens and data acquisition}

Two formalin-fixed whole-brains (specimen 1 and specimen 2) and one formalin-fixed left hemisphere specimen (specimen 3), acquired from subjects without known neurological or psychiatric diseases, were used in this study. Specimen 1 was from a donor giving informed consent under the Maastricht University, Department of Anatomy and Embryology body donation program (post mortem interval to fixation: $12 \mathrm{~h}$, fixation to scanning: 30 months) as regulated by the Dutch law for the use of human remains for scientific research and education ("Wet op de Lijkbezorging"). Specimen 2 was obtained from the University of Rostock body donor program (post mortem interval to fixation: $32 \mathrm{~h}$, fixation to scanning: 25 months). Specimen 3 (post mortem interval to fixation: $3.5 \mathrm{~h}$, fixation to scanning: 3 months) was borrowed from Dr. R.N. Kooijmans (NIN/KNAW), and originally obtained from The Netherlands Brain Bank (NBB - NHB1037 - 121/2018), Netherlands Institute for Neuroscience, Amsterdam (open access: www. brainbank.nl). All material has been collected from donors for or from whom a written informed consent for a brain autopsy and the use of the material and clinical information for research purposes had been obtained by the NBB. Each specimen was immersed in its fixation solution and then enclosed in a $3 \mathrm{D}$ conformal container printed using a watertight, chemically resistant material SOMOS XC11122 (DSM Heerlen) [174]. The specimen vary quite strongly in their white matter $\mathrm{T}_{1}$, $\mathrm{T}_{2}$ and diffusivity $\mathrm{D}$ parameters, as a consequence of their post mortem interval (PMI), fixation time and general tissue quality, discussed in more detail elsewhere [59, 147, 175, 197]. Specimen 1 had a white matter $\mathrm{T}_{1} / \mathrm{T}_{2} / \mathrm{D}$ average of approximately $0.350 \mathrm{~s} / 0.009 \mathrm{~s} / 2.0 \times 10^{-4} \mathrm{~mm}^{2} / \mathrm{s}$, which is relatively low for fixed ex vivo brain specimen. Specimen 2 and specimen 3 had a white matter $\mathrm{T}_{1} / \mathrm{T}_{2} / \mathrm{D}$ average of approximately $0.550 \mathrm{~s} / 0.018 \mathrm{~s} / 2.2 \times 10^{-4}$ $\mathrm{mm}^{2} / \mathrm{s}$, which are relatively high for fixed ex vivo brain specimen. These differences, particularly in $\mathrm{T}_{2}$ and $\mathrm{D}$, served to investigate the performance of $\mathrm{k}_{\mathrm{T}}$-dSTEAM over a reasonably expected range of tissue quality. 
The $\mathrm{k}_{\mathrm{T}}$-dSTEAM sequence was implemented on a $9.4 \mathrm{~T}, 82 \mathrm{~cm}$ bore Siemens MAGNETOM research scanner (Siemens Healthineers, Erlangen, Germany) with maximum gradient amplitude of $80 \mathrm{mT} / \mathrm{m}$ and maximum slew rate of $330 \mathrm{mT} / \mathrm{m} / \mathrm{ms}$ per physical axis. For RF transmission and reception, a custom-built $9.4 \mathrm{~T}$ whole-brain coil $(400 \mathrm{MHz})$ with 24 receive channels and 8 transmit channels was used [174]. In this coil, the 24 receive coil loops are laid out as a phased array, conformal and tightly fitting to the brain container, with on-coil preamplifier circuits and decoupled by geometric overlap and preamplifier decoupling. The eight separate transmit loops have a $2 \times 2 \times 2$ layout, placed on 2 separate hemispheric formers (4 transmit channels in a $2 \times 2$ pattern on each half), allowing for full $3 \mathrm{D}$ parallel transmission.

The composite $\mathrm{k}_{\mathrm{T}}$-point pulses in $\mathrm{k}_{\mathrm{T}}$-dSTEAM are created analogous to the composite $\mathrm{k}_{\mathrm{T}}$-point excitation pulse in GRE sequences [53, 208]. Each one is a globally non-selective composite pulse with 8 to 16 sub-pulses, optimised for $\mathrm{B}_{1}{ }^{+}$homogeneity (Figure 3.2). For the $\mathrm{k}_{\mathrm{T}}$-points pulse design, a $\mathrm{B}_{0}$ map [57] and transmit $\mathrm{RF}$ profile $\left(\mathrm{B}_{1}{ }^{+}\right)$map for each of the transmit channels were acquired with transmitting phase-encoded by using a $\mathrm{T}_{2}$ and $\mathrm{T}_{2}{ }^{*}$ compensated version of DREAM [152]. $\mathrm{B}_{0}$ shimming and $\mathrm{k}_{\mathrm{T}}$-point pulse calculation for $\mathrm{B}_{1}{ }^{+}$shimming was performed by the approach established in the references [181] and [208], using custom-written MATLAB routines (MathWorks, MA, USA). For the $\mathrm{k}_{\mathrm{T}}$-points pulses, the sub-pulse spacing was set to $180 \mu \mathrm{s}$ for all three composite pulses. The excitation and recalling pulses used 8 sub-pulses, whereas the storing pulse was implemented with 16 composite sub-pulses as shown in the following references [72] and [73].

After $\mathrm{B}_{0}$ shimming and composite $\mathrm{RF}$ pulse calculations, $\mathrm{k}_{\mathrm{T}}$-dSTEAM images were acquired for $T_{1}$ and $T_{2}$ relaxometry, and diffusion analysis. The acquisition parameters per study are specified in Table 3.3. The imaging was performed using a segmented 3D EPI readout. All scans were acquired at the same field of view $(\mathrm{FoV})$ of $162 \times 150 \mathrm{~mm}^{2}$ in sagittal orientation, readout along the anterior-posterior (AP) direction (parallel to the scanner bore) with an EPI-factor of 5. All diffusion acquisitions used for modelling (for parameters see Table 3.3) were acquired with a minimum of 24 directions per shell and accompanied by 3 or $4 b_{0}$-weighted volumes per shell. The crusher gradients around the storing and recalling pulses in these $b_{0}$ volumes accumulated an actual b-value approximately $200 \mathrm{~s} / \mathrm{mm}^{2}$, which we therefore also refer to as low b-value or low-b. In some of the acquisitions, a factor of two undersampling was applied along the partition encoding dimension (2x PA) in the left-right (LR) direction of the brain. 
In those cases, a low-resolution reference image (auto-calibration signal or ACS image) was acquired using the same sequence and parameters but at $2 \mathrm{~mm}$ isotropic resolution and full encoding, for subsequent offline reconstruction in Matlab. Noise-only scans were also acquired in order to estimate the channel-by-channel noise covariance matrix [119].

\begin{tabular}{|c|c|c|c|c|c|c|}
\hline $\begin{array}{l}\text { Measurement } \\
\text { (Specimen) }\end{array}$ & $\begin{array}{c}\text { Resolution }[\mu \mathrm{m}] \\
\text { (Acquisition } \\
\text { scheme) }\end{array}$ & $\begin{array}{c}\text { TR } \\
{[\mathrm{sec}]}\end{array}$ & TE [sec] & $\mathrm{TM}[\mathrm{sec}]$ & $\begin{array}{l}\text { b-values } \\
{\left[\mathrm{s} / \mathrm{mm}^{2}\right] \text { (\# }} \\
\text { directions) }\end{array}$ & $\begin{array}{c}\text { Acquisition } \\
\text { time (per } \\
\text { volume/total) }\end{array}$ \\
\hline $\begin{array}{l}\mathrm{T}_{1} \text { relaxometry } \\
\text { (Specimen 1) }\end{array}$ & $\begin{array}{c}1000 \\
\text { EPI } 5-2 x \text { PA }\end{array}$ & 1.000 & 0.014 & $\begin{array}{c}0.060: 0.030: \\
0.420\end{array}$ & Not applicable & $\begin{array}{c}00: 33: 37 / \\
05: 36: 10\end{array}$ \\
\hline $\begin{array}{c}\mathrm{T}_{2} \text { relaxometry } \\
\text { (Specimen 1) }\end{array}$ & $\begin{array}{c}1000 \\
\text { EPI } 5-2 x \text { PA }\end{array}$ & 0.350 & $\begin{array}{l}0.014,0.016: \\
0.004: 0.044\end{array}$ & 0.120 & Not applicable & $\begin{array}{c}00: 11: 46 / \\
01: 45: 54\end{array}$ \\
\hline $\begin{array}{c}\text { Diffusion } \\
\text { (Specimen 1) }\end{array}$ & $\begin{array}{l}1000 \\
\text { EPI } 5\end{array}$ & 0.350 & 0.025 & $0.134,0.205$ & $\begin{array}{c}2000(1), 4000(36) \\
6000(48)\end{array}$ & $\begin{array}{c}00: 23: 35 / \\
36: 56: 50\end{array}$ \\
\hline $\begin{array}{c}\text { Diffusion } \\
\text { (Specimen 1) }\end{array}$ & $\begin{array}{c}500 \\
\text { EPI } 5-2 \times \text { PA }\end{array}$ & 0.350 & 0.026 & $0.065,0.135$ & $2063(24), 4034(36)$ & $\begin{array}{c}00: 53: 49 / \\
60: 05: 43\end{array}$ \\
\hline $\begin{array}{c}\text { Diffusion } \\
\text { (Specimen 2) }\end{array}$ & $\begin{array}{l}1000 \\
\text { EPI } 5\end{array}$ & 0.350 & 0.025 & $0.065,0.135$ & $\begin{array}{l}2000(1), 4000(1) \\
6000(1), 8000(1)\end{array}$ & $\begin{array}{c}00: 23: 35 / \\
01: 34: 20\end{array}$ \\
\hline $\begin{array}{c}\text { Diffusion } \\
\text { (Specimen 2) }\end{array}$ & $\begin{array}{l}400 \\
\text { EPI } 5\end{array}$ & 0.350 & 0.027 & 0.096 & $3000(20)$ & $\begin{array}{c}02: 23: 44 / \\
52: 42: 08\end{array}$ \\
\hline $\begin{array}{l}\mathrm{T}_{1} \text { relaxometry } \\
\text { (Specimen } 3)\end{array}$ & $\begin{array}{l}1000 \\
\text { EPI } 5\end{array}$ & 0.450 & 0.025 & $\begin{array}{c}0.050,0.120: \\
0.060: 0.360 \\
0.450\end{array}$ & Not applicable & $\begin{array}{c}00: 17: 17 / \\
02: 00: 59\end{array}$ \\
\hline $\begin{array}{l}\text { Diffusion (PGSE) } \\
\text { (Specimen 3) }\end{array}$ & $\begin{array}{l}1000 \\
\text { EPI } 5\end{array}$ & 0.450 & $0.054,0.061$ & $\begin{array}{c}(\delta, \Delta):(0.022 \\
0.025),(0.026 \\
0.029),(0.029 \\
0.031)\end{array}$ & $4059(36), 6082(1)$ & $\begin{array}{c}00: 17: 17 / \\
11: 48: 37\end{array}$ \\
\hline $\begin{array}{l}\text { Diffusion (STEAM) } \\
\text { (Specimen 3) }\end{array}$ & $\begin{array}{l}1000 \\
\text { EPI } 5\end{array}$ & 0.450 & 0.025 & $\begin{array}{c}0.134,0.205 \\
0.205\end{array}$ & $\begin{array}{r}4059(36), 4059 \\
(36), 6085(48)\end{array}$ & $\begin{array}{c}00: 17: 17 / \\
38: 18: 41\end{array}$ \\
\hline
\end{tabular}

Figure 3.3: $\mathrm{k}_{\mathrm{T}}$-dSTEAM relaxometry and diffusion acquisitions parameters and specimens used in this study. All acquisitions were performed using isotropic resolution. For diffusion, each b-value corresponds to a single mixing time (longer TM's for higher b-values). A sequence of timing parameters, such as TM or TE, are described as low:step:high. The acquisition time, on the other hand, is referred to as hrs:min:sec. Total acquisition times includes all volumes (for diffusion, including $b_{0}$ images). In the case of PGSE, the TM column reports the diffusion gradient duration $(\delta)$ and diffusion time $(\Delta)$ used per acquired b-value as indicated in the relevant PGSE cell. 2x PA: a factor of two undersampling was applied along the partition encoding (3D phase encoding) dimension.

For the purpose of comparing varying-TM multi-shell acquisition to same- 
TM multi-shell acquisition we acquired, for specimen 3, a short TM $\mathrm{b}=$ $4000 \mathrm{~s} / \mathrm{mm}^{2}(\mathrm{~b} 4 \mathrm{k})$ shell and long TM $\mathrm{b}=6000 \mathrm{~s} / \mathrm{mm}^{2}(\mathrm{~b} 6 \mathrm{k})$ shell and added a matched long TM b4k shell. To show the achievable advantages of $\mathrm{k}_{\mathrm{T}^{-}}$ dSTEAM over PGSE in this study, a matched set of diffusion acquisitions at different $b$-values were acquired on the same specimen as specified in Table 3.3. For comparison purposes, the diffusion gradient amplitude (G) was set to the maximum possible $(80 \mathrm{mT} / \mathrm{m})$ for both sequences so that $\mathrm{TE}$ could be minimised. To avoid $\mathrm{B}_{1}{ }^{+}$inhomogeneity in the PGSE acquisitions, the $\mathrm{k}_{\mathrm{T}}$-dSTEAM was converted to $\mathrm{k}_{\mathrm{T}}$-PGSE accordingly, by changing the phase (from $\mathrm{x}$ to $\mathrm{z}$ ) and flip angle (from $\pi / 2$ to $\pi \mathrm{rad}$ ) of the storing pulse and removing the recalling pulse. This allowed for the use of the same composite pulses to achieve the same level of $\mathrm{B}_{1}{ }^{+}$homogenisation.

\subsubsection{Image reconstruction and analysis}

All the acquired images were reconstructed offline in MATLAB. EPI ghost correction (by odd-even echo offsets) were corrected using phase navigators [164] and Inverse Fourier transforms were applied without windowing along all axes. Accelerated undersampled relaxometry $\mathrm{T}_{1} \mathrm{w}$ and $\mathrm{T}_{2} \mathrm{~W}$ data were reconstructed using ESPIRiT [209] as implemented in the BART toolbox [198], using the low-resolution reference image and the noise covariance matrix. For diffusion-weighted data, covariance-weighted root sum-of-squares (rCovSos) reconstruction [119, 207] was used for channel combination to achieve a superior SNR compared to unweighted root sumof-squares (rSoS).

After offline image reconstruction, several analyses were performed. Signalto-noise (SNR) comparisons between $\mathrm{k}_{\mathrm{T}}$-PGSE and $\mathrm{k}_{\mathrm{T}}$-dSTEAM were made for equal b-values (4000 s/mm ${ }^{2}$ and $6000 \mathrm{~s} / \mathrm{mm}^{2}$ ), as well as between $\mathrm{k}_{\mathrm{T}^{-}}$ dSTEAM volumes at different b-values and TM's $\left(4000 \mathrm{~s} / \mathrm{mm}^{2}\right.$ at TM of $0.134 \mathrm{~s}, 4000 \mathrm{~s} / \mathrm{mm}^{2}$ at TM of $0.205 \mathrm{~s}$ and $6000 \mathrm{~s} / \mathrm{mm}^{2}$ at TM of 0.205 s). Image SNR values were calculated as mean(signal)/std.dev(noise) over spherical volumes of interest (VOIs), 9 voxels in diameter. The mean signal was estimated in different areas in specimen 3 and the noise standard deviation was obtained using 4 VOIs in signal-free image background regions.

All quantitative $\mathrm{T}_{1}\left(\mathrm{qT}_{1}\right)$ and $\mathrm{T}_{2}\left(\mathrm{qT}_{2}\right)$ and diffusion model fitting from the $\mathrm{k}_{\mathrm{T}}$-dSTEAM data were performed using the Microstructure Diffusion Toolkit (MDT, https://github.com/cbclab/MDT) by nonlinear GPU accelerated optimisation [101]. Powell optimisation with Patience 20 was em- 
ployed for $\mathrm{qT}_{1}$ and $\mathrm{qT}_{2}$ estimation using Equation 3.2. Unweighted signal $\left(\mathrm{S}_{0}\right)$ in Equation 3.2 was initialised using the highest measured signal (usually the lowest TE or TM) and an offset-Gaussian likelihood function was used to account for the Rician rectified noise floor. The noise level was estimated by selecting a region of interest (ROI) in the signal-free image background and calculating its complex standard deviation by assuming a Rayleigh distribution [95]

Multi-shell $\mathrm{k}_{\mathrm{T}}$-dSTEAM data were analysed both separately as single shells (of b4k and b6k), and jointly as multi-shell data using the same TM (sameTM, in specimen 3) and varying TM (varying-TM, in specimen 1 and 3). Single-shell data was analysed with Diffusion Tensor Imaging (DTI) and Ball\&Stick (B\&S) with one $\left(B \& S_{r 1}\right)$ and two $\left(B \& S_{r 2}\right)$ sticks models using Equation 3.6. The diffusivity for the stick compartment was fixed to the mean diffusivity obtained from the corresponding DTI analysis. For same-TM multi-shell data, the analysis was identical to the one used for single-shell analysis (i.e. using Equation 3.6). The analysis for varying-TM multi-shell data was performed in a 5-step cascade approach summarised in Figure 3.4. First, the $\mathrm{qT}_{1}$ obtained from relaxometry fitting (step 1, Figure 3.4) was used as a fixed $\mathrm{T}_{1}$ value to disentangle the $\mathrm{T}_{1}$ and diffusion contributions to the signal between shells using Equation 3.5 (steps 2 to 4, Figure 3.4). Finally, Diffusion models $B \& \mathrm{~S}_{\mathrm{r} 1}$ and $\mathrm{B} \& \mathrm{~S}_{\mathrm{r} 2}$ were estimated with fixed $\mathrm{S}_{0}$ and $\mathrm{T}_{1}$ contributions (step 5, Figure 3.4). Again, Powell optimisation with patience 20 was employed for all the cases. After modelling, a voxel-wise model selection of the optimal number of stick orientations was performed $\left(B \& \mathrm{~S}_{\mathrm{r} 2}\right.$ selected) by selecting voxel-wise the model corresponding to the lowest Bayesian Information Criteria (BIC). In addition, if the stick fraction was lower than $10 \%$ (for $\mathrm{stick}_{0}$ ) and $17.5 \%$ (for stick ${ }_{1}$ ) during model optimisation then the diffusivity is considered as null to help avoid multiplicity in the stick orientations. 

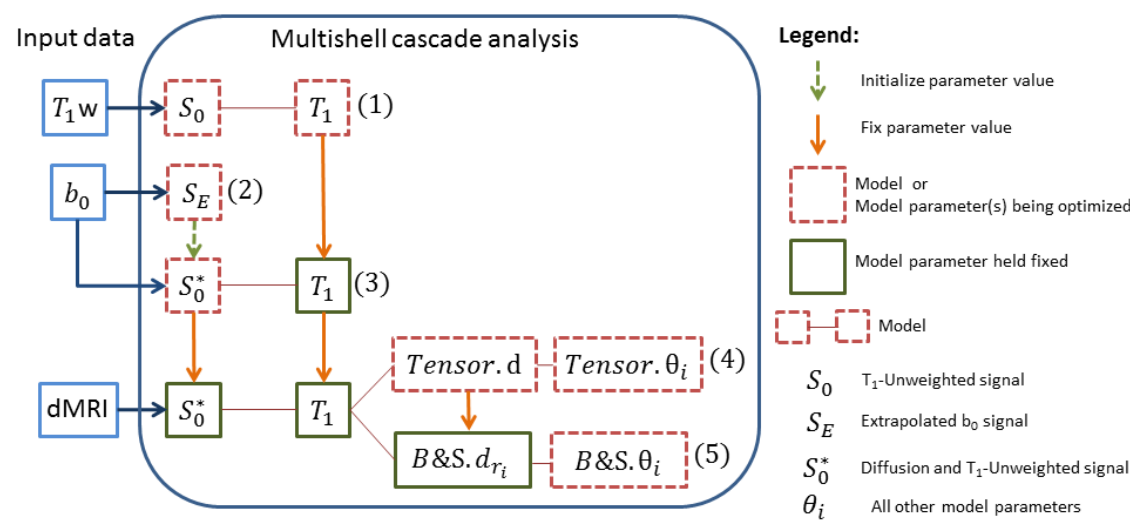

Figure 3.4: $\mathrm{k}_{\mathrm{T}}$-dSTEAM cascade data analysis using nonlinear multi-shell diffusion model optimisation. Parameters in more complex diffusion models are initialised with or held fixed at, values from simpler models (steps 4-5). Simpler models are estimated from separate $\mathrm{T}_{1}$ relaxometry data (step 1 ) and the $\mathrm{b}_{0}$ volumes (step 2 and 3 ).

As an illustration of the resolution effects on tractography, deterministic streamline tractography was performed on the DTI modelling results of the $1000 \mu \mathrm{m}$ and $400 \mu \mathrm{m}$ whole-brain volumes (specimen 1 and specimen 2 , respectively). Simple deterministic DTI tractography was chosen to highlight the effect of data resolution, rather than tractography algorithm sophistication. The tractography parameters used for both resolutions were: propagation direction by the truncated tensor projection operator $[173,226]$, trilinear interpolation, Euler integration, fractional anisotropy threshold of 0.1 , step size of $0.4 \mathrm{~mm}$ and angle threshold of $35^{\circ}$ leading to minimum radius of curvature of $0.5729 \mathrm{~mm}$. Tractography was performed across the whole-brain and seeded using a WM mask in a $5 \times 5 \times 5$ grid per $1000 \mu \mathrm{m}$ voxel and a $2 \times 2 \times 2$ grid per $400 \mu \mathrm{m}$ voxel to match seeding density.

To compare deterministic crossing fibre tractography in the cerebellar peduncle between the single-shell $B \& \mathrm{~S}_{\mathrm{r} 2}$ model, multi-shell $\mathrm{B} \& \mathrm{~S}_{\mathrm{r} 1}$ model and multi-shell $B \& S_{\mathrm{r} 2}$ model, the same tractography parameters were used in the $1000 \mu \mathrm{m}$ resolution data of specimen 1, except for: propagation direction by selection of the most collinear local direction, 4th-order RungeKutta integration and angle threshold of $30^{\circ}$. Cerebellar peduncle tractography was seeded using a box mask in a $3 \times 3 \times 3$ grid per $1000 \mu \mathrm{m}$ voxel covering the cerebellar peduncle volume and exclusion masks were 
positioned $5 \mathrm{~mm}$ below and $5 \mathrm{~mm}$ above the seeding mask to exclude the cortico-spinal tract (CST) streamlines.

\subsection{Results}

The results are organised as follows. We first validate the predicted improvement of $\mathrm{k}_{\mathrm{T}}$-dSTEAM over more conventional PGSE dMRI in the largesample-large-bore case, through a comparative study between $\left(\mathrm{k}_{\mathrm{T}}\right)$-PGSE and $k_{T^{-}}$-dSTEAM. Subsequently, we use the superior SNR efficiency of $k_{T^{-}}$ dSTEAM for ultra-high resolution whole-brain diffusion MRI. We then show how this sequence can be conveniently used for quantitative $\mathrm{T}_{1}$ and $\mathrm{T}_{2}$ mapping, by relaxometry over varying TE's and TM's. This is a prerequisite for multi-shell diffusion acquisition and multi-orientation analysis, which we focus on in the final subsection.

\subsubsection{Comparative study between $\left(\mathrm{k}_{\mathrm{T}}\right)$-PGSE and $\mathrm{k}_{\mathrm{T}}$-dSTEAM}

To compare the SNR efficiency of $\mathrm{k}_{\mathrm{T}}$-dSTEAM to that of a conventional PGSE acquisition, a matched set of diffusion acquisitions at different $b$ values were acquired on the same specimen. The same TR and maximum diffusion gradient amplitude was used with minimised TE (see Table 3.3). Furthermore, the same $\mathrm{k}_{\mathrm{T}}$-points $\mathrm{B}_{1}{ }^{+}$homogenisation was used in the PGSE acquisitions, which we therefore refer to as $\mathrm{k}_{\mathrm{T}}$-PGSE. Figure 3.5 shows the $\mathrm{k}_{\mathrm{T}}$-PGSE vs $\mathrm{k}_{\mathrm{T}}$-dSTEAM comparison for specimen 3 at $\mathrm{b}$-values of 4000 and $6000 \mathrm{~s} / \mathrm{mm}^{2}$. For both b-values, $\mathrm{k}_{\mathrm{T}}$-dSTEAM images qualitatively show higher signal in comparison to $\mathrm{k}_{\mathrm{T}}$-PGSE (Figure 3.5A). This is corroborated quantitatively by the estimated SNR in three different areas along the specimen (figure 3.5B). In the areas where the signal is high (red ROI) or moderately high (green ROI), the SNR for $\mathrm{k}_{\mathrm{T}}$-dSTEAM is over $40 \%$ higher than for $\mathrm{k}_{\mathrm{T}}$-PGSE, and over $20 \%$ higher in the low signal area (blue ROI). In ROI 2 and 3, but not ROI 1, the $\mathrm{k}_{\mathrm{T}}$-dSTEAM SNR improvement shows a tendency to increase from $b=4000 \mathrm{~s} / \mathrm{mm}^{2}$ to $\mathrm{b}=6000 \mathrm{~s} / \mathrm{mm}^{2}$. Note that the SNR's reported here are for diffusion-weighted single volumes (arguably the worst-case SNR), whereas in the literature the SNR for $b_{0}$ volumes is often reported (arguably the best-case SNR).

To assess the effects of SNR differences on dMRI analysis, figure 3.6 shows the mean $\mathrm{b}_{0}\left(\mathrm{~S}_{0}\right.$, figure $\left.3.6 \mathrm{~A}\right)$ and fractional anisotropy (FA, figure 3.6B) parameter maps from DTI model fitting for the $\mathrm{k}_{\mathrm{T}}$-PGSE and $\mathrm{k}_{\mathrm{T}}$-dSTEAM data at the same b-value of $b=4000 \mathrm{~s} / \mathrm{mm}^{2}(\mathrm{~b} 4 \mathrm{k})$. The estimated $\mathrm{S}_{0}$ for 


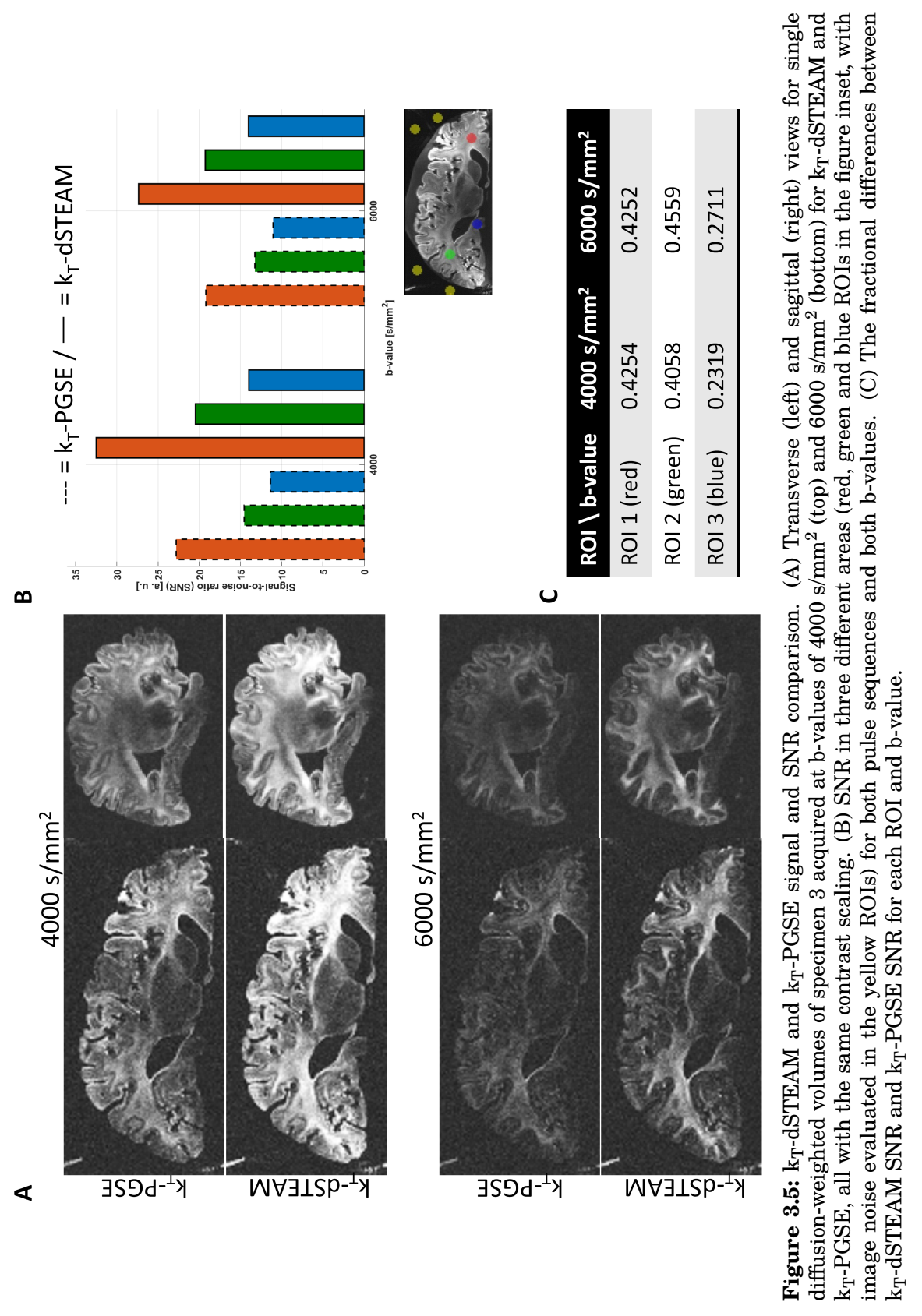


$\mathrm{k}_{\mathrm{T}}$-PGSE is lower along the entire specimen than the $\mathrm{S}_{0}$ for $\mathrm{k}_{\mathrm{T}}$-dSTEAM, reflecting similar signal level differences as the diffusion-weighted volumes in Figure 3.5. The FA maps show even clearer differences with lower and noisier FA estimates for $\mathrm{k}_{\mathrm{T}}$-PGSE, reflecting the known downward bias in FA estimates caused by low SNR [115].

\subsubsection{Ultra-high resolution whole-brain diffusion MRI}

Figure 3.7 shows single volumes of whole-brain $\mathrm{k}_{\mathrm{T}}$-dSTEAM acquisitions for both the low- $\mathrm{T}_{2}$ specimen 1 (Figure 3.7A) and the high- $\mathrm{T}_{2}$ specimen 2 (Figure 3.7B) at two different b-values (low and high) and two resolutions (high and ultra-high). In the low-b acquisitions, the homogeneous highSNR STEAM signal can be appreciated, as well as the considerable increase in visible detail in going from the high (for whole-brain diffusion acquisitions) resolution of $1000 \mu \mathrm{m}$ isotropic to $8 \mathrm{x}(500 \mu \mathrm{m}$ for specimen 1$)$ or $16 \mathrm{x}(400 \mu \mathrm{m}$ for specimen 2$)$ higher isotropic resolution. The high b-value acquisitions (separately windowed and levelled) at $1000 \mu \mathrm{m}$ isotropic show the effect of diffusion-weighting using moderate b-values. At the much lower diffusivities in fixed post mortem tissue, the contrast achieved with these $b$-values is more subtle and dependent on the varying tissue diffusivity between samples. Notably, the WM contrast achieved with $b=3000$ $\mathrm{s} / \mathrm{mm}^{2}$ in the high- $\mathrm{T}_{2} /$ high diffusivity specimen 2 is qualitatively higher than that achieved with $\mathrm{b}=4000 \mathrm{~s} / \mathrm{mm}^{2}$ in the low- $\mathrm{T}_{2} /$ low diffusivity specimen 1. Finally, the high-b ultra-high resolution acquisitions show that the $\mathrm{k}_{\mathrm{T}}$-dSTEAM sequence is capable of producing imaging volumes in this extremely SNR-challenged regime, although there exists a clear trade-off in expending SNR towards higher diffusion-weighting or higher resolution. Nevertheless, these images have sufficient SNR for diffusion imaging and tractography analysis. 


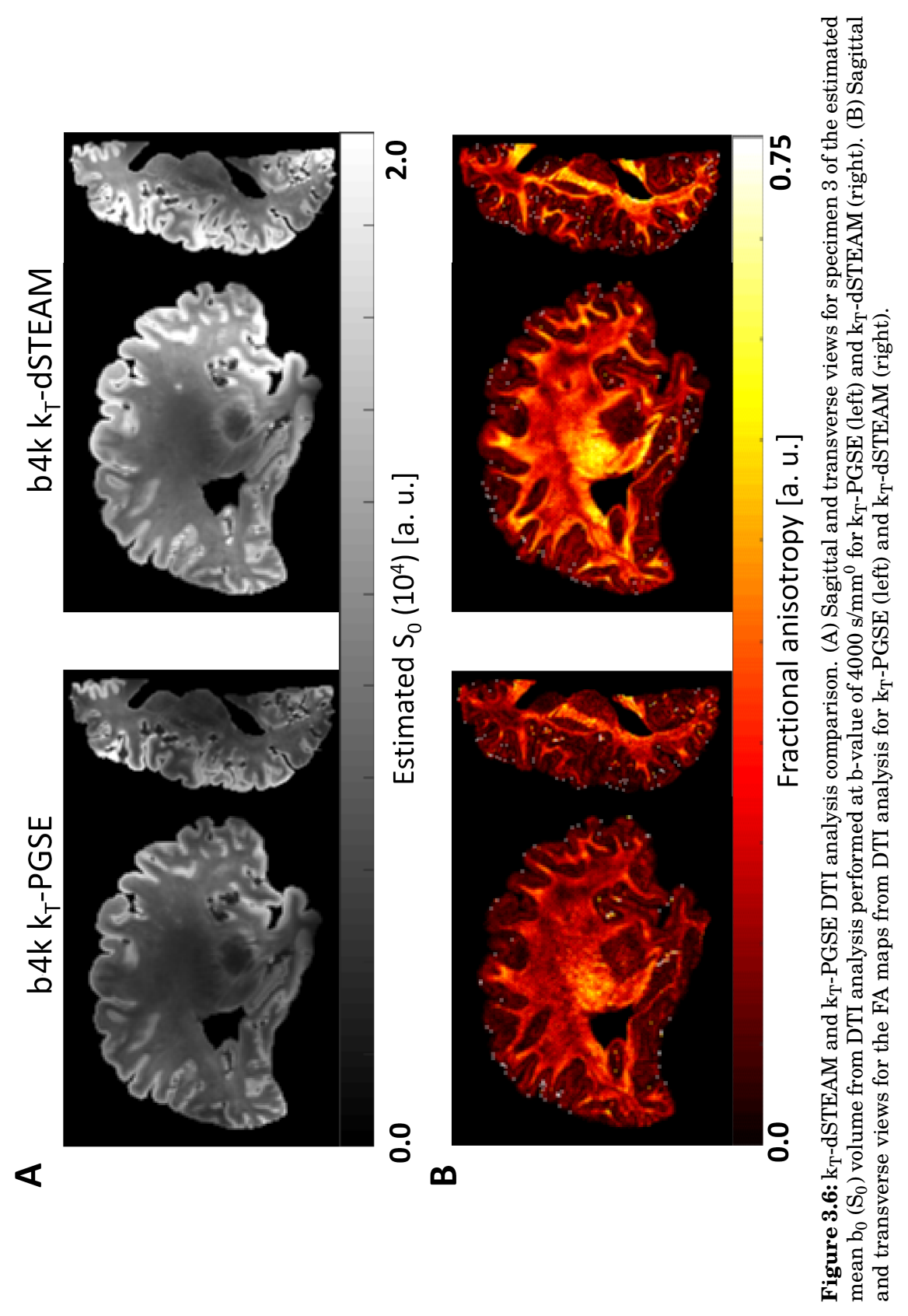



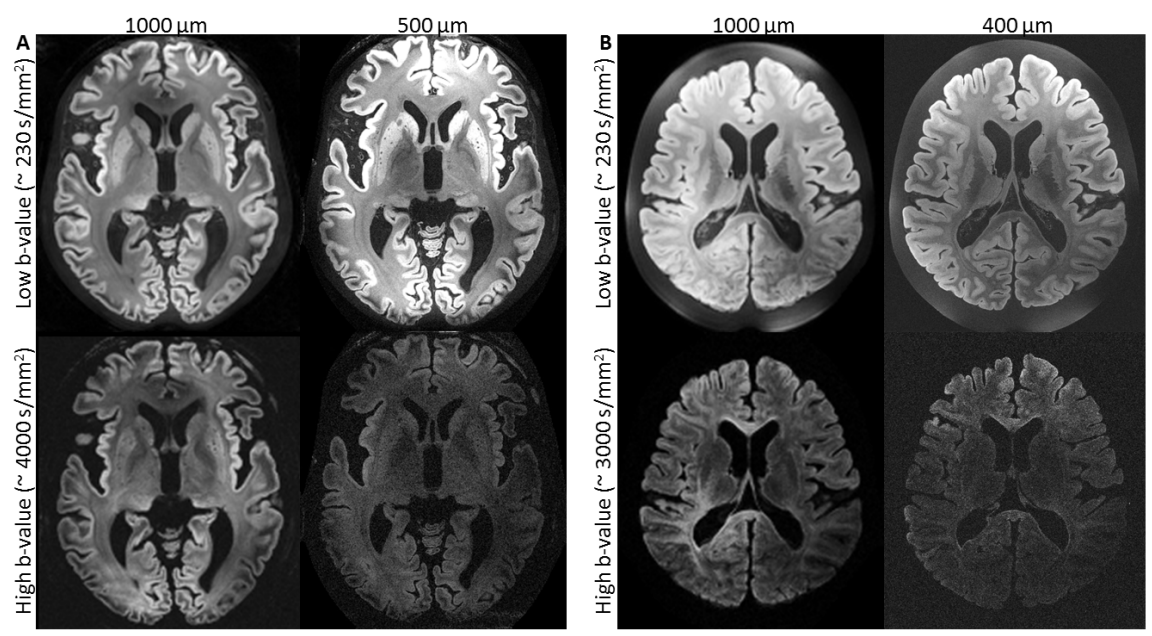

Figure 3.7: Single diffusion direction volumes of $\mathrm{k}_{\mathrm{T}}$-dSTEAM whole-brain acquisitions. (A) Mid-transverse views at high $(1000 \mu \mathrm{m})$ and ultra-high $(500 \mu \mathrm{m})$ resolution for specimen 1 for low (top) and high (bottom) b-values. (B) Mid-transverse views at high (1000 $\mu \mathrm{m})$ and ultra-high $(400 \mu \mathrm{m})$ resolution for specimen 2 for low (top) and high (bottom) b-values.

Figure 3.8 shows $400 \mu \mathrm{m}$ single-shell results using DTI and B\&S $\mathrm{S}_{\mathrm{r} 1}$ models. The stick fraction map (Figure 3.8A) displays good contrast between WM and GM, even in deep brain nuclei, as well as a tendency for a higher stick fraction in dense tracts such as the corpus callosum (CC) and CST. DTI primary eigenvectors (Figure 3.8B) define both large WM tracts, such as CC, CST, and Cingulum (Cg), as well as radial directions in the GM at high spatial fidelity. The high resolution could potentially support tractography of the high curvature insertions (and exits) of white matter projections into (and out of) the cortex. Tractography at standard in vivo resolutions is known to be biased towards connecting gyral crowns, but not sulcal walls through white matter, which does not reflect known neuroanatomy [212]. 


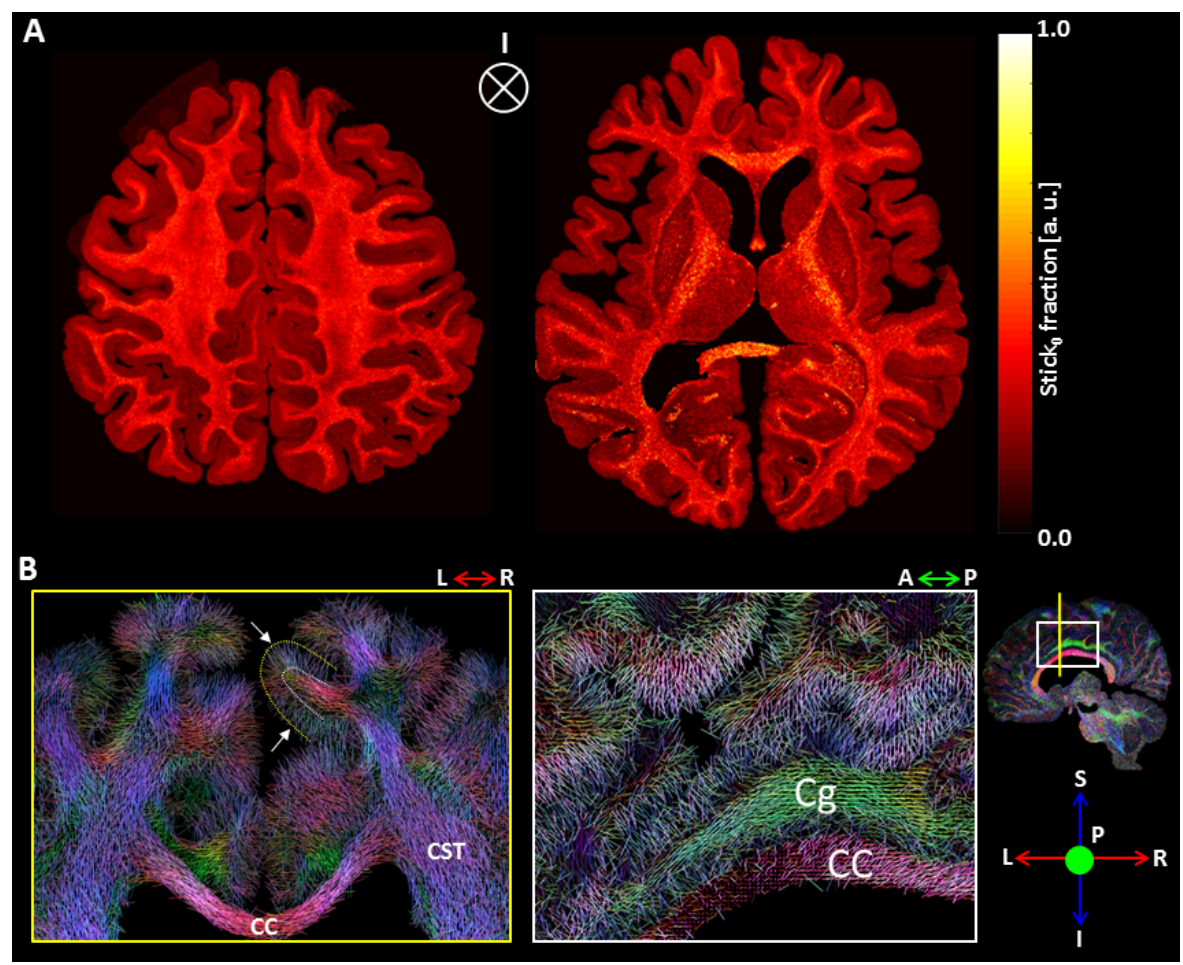

Figure 3.8: $\mathrm{k}_{\mathrm{T}} \mathrm{T}$-dSTEAM single-shell $400 \mu \mathrm{m}$ analysis results for specimen 2 . A) Two axial slices through the Stick $_{0}$ fraction map resulting from a Ball\& Stick $\mathrm{r}_{\mathrm{r}}$ model fit. B) Direction colour coded DTI primary eigenvector in coronal (left) and sagittal (right) slices, the position indicated in the inset on the far right (and the direction colour encoded in coronal view). Radial directions in the GM are indicated by the white arrows, between WM/GM boundary (dashed white line) and pial boundary (dashed yellow line). CC: Corpus Callosum, Cg: Cingulum, CST: Cortico-Spinal Tract.

Figure 3.9 shows a comparison between tractography near the white/grey matter border in a gyral crown performed at high (1000 $\mu \mathrm{m}$ isotropic) and ultra-high (400 $\mathrm{mm}$ isotropic) resolutions. Using the same deterministic tractography parameters for both resolutions, streamlines can be tracked beyond the WM/GM boundary. However, at the ultra-high resolution, the streamlines show less of the unwanted gyral crown bias, and more highcurvature paths connecting part of the sulcal wall through white matter. 


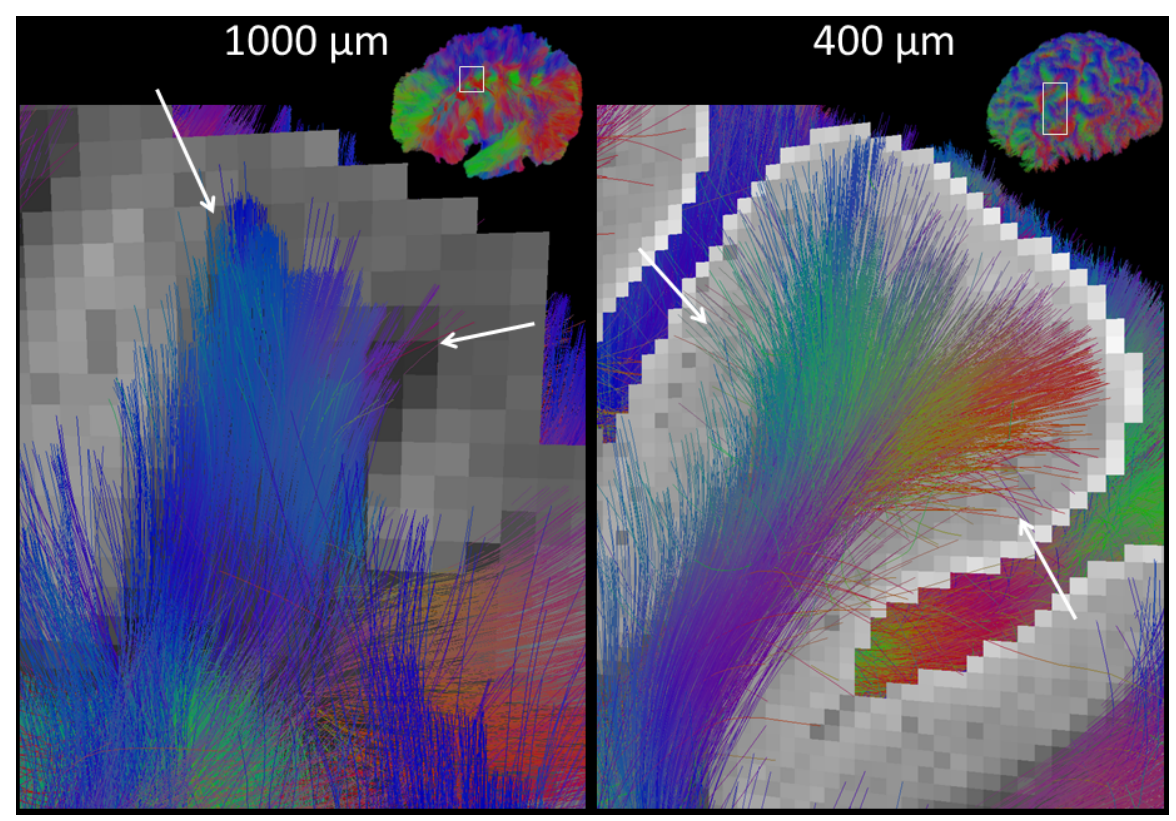

Figure 3.9: $\mathrm{k}_{\mathrm{T}}$-dSTEAM diffusion tractography of the whole-brain at high $(1000 \mu \mathrm{m}$, left) and ultra-high ( $400 \mu \mathrm{m}$, right) resolution. whole-brain tractography is zoomed in to a gyral crown in frontal cortex indicated by the white box in the upper right inset. The arrows indicate, for both datasets, the approximate extreme extents of the gyral grey matter connectivity through white matter, which extends further into the sulcal wall at the higher resolution.

\subsubsection{Relaxometry for quantitative $T_{1}$ and $T_{2}$ mapping}

STEAM sequence in general, and $\mathrm{k}_{\mathrm{T}}$-dSTEAM in particular, are highly suitable for both quantitative $\mathrm{T}_{1}$ and $\mathrm{T}_{2}$ mapping by relaxometry on $\mathrm{TM}$ and TE respectively. Figure 3.10 shows relaxometry data and corresponding quantitative $\mathrm{T}_{1}\left(\mathrm{qT}_{1}\right)$ and $\mathrm{T}_{2}\left(\mathrm{qT}_{2}\right)$ maps for specimen 1 , estimated using Equation 3.2. Fitted $\mathrm{T}_{1}$ values were between about 0.3 and $0.7 \mathrm{sec}$ with no clear difference in $\mathrm{qT}_{1}$ distribution between $\mathrm{GM}$ and $\mathrm{WM}$, as is also clear from the visual lack of contrast in the $\mathrm{qT}_{1}$ map (Figure 3.10A). On the other hand, $\mathrm{qT}_{2}$ values show a clear contrast between GM and WM, where the GM qT $\mathrm{T}_{2}$ values had a mode of $0.011 \mathrm{sec}$ and $\mathrm{WM} \mathrm{qT}_{2}$ values had a mode of $0.009 \mathrm{sec}$, as indicated by the histograms in Figure 3.10D. This agrees with the observations that little $\mathrm{T}_{1}$ contrast remains between grey and white matter ex vivo and that, rather than $\mathrm{T}_{2} / \mathrm{T}_{2}{ }^{*}$ contrast, dominates 
the ex vivo image [14].
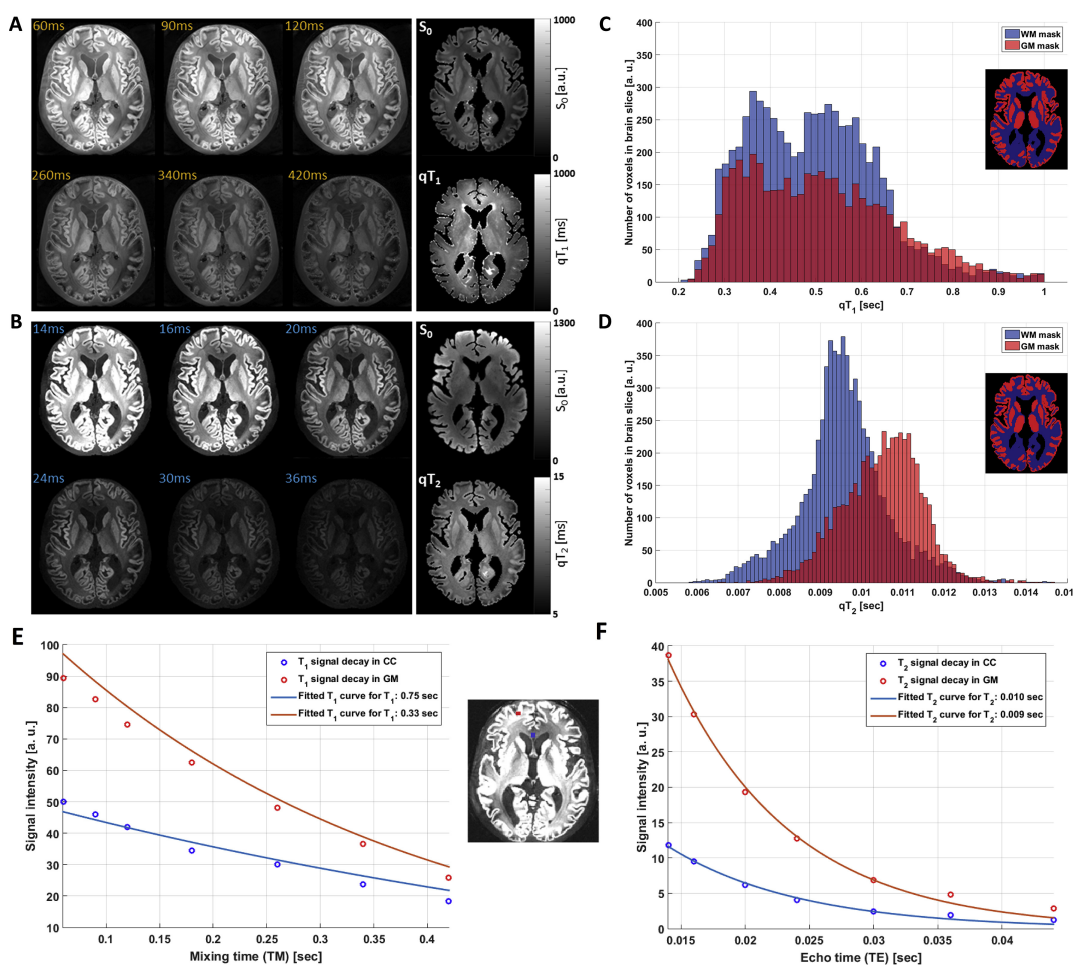

Figure 3.10: $\mathrm{k}_{\mathrm{T}}$-dSTEAM relaxometry data and corresponding quantitative $\mathrm{T}_{1}\left(\mathrm{q}_{1}\right)$ and $\mathrm{T}_{2}\left(\mathrm{qT}_{2}\right.$ ) maps for specimen 1. (A) Volumes at increasing mixing times showing $\mathrm{T}_{1}$ signal decay (left), the corresponding $\mathrm{qT}_{1}$ map from model fitting (middle), and (C) histograms of $\mathrm{T}_{1}$ values along the slice separately for GM and WM masks (right). (B) Volumes at increasing echo times showing $\mathrm{T}_{2}$ signal decay (left), the corresponding $\mathrm{qT}_{2}$ maps from model fitting (middle), and (D) histograms of $\mathrm{T}_{2}$ values along the slice separately for GM and WM masks (right). In contrast to the $\mathrm{qT}_{1}$ histograms, $\mathrm{qT}_{2}$ shows two peaks corresponding to WM and GM structures. (E) and ( $\mathrm{F}$ ) show the experimental $\mathrm{T}_{1}$ and $\mathrm{T}_{2}$ signal decay (circles) for the corpus callosum (CC, blue) and GM (red) and their corresponding $\mathrm{T}_{1}$ and $\mathrm{T}_{2}$ signal fitting (solid lines) respectively.

\subsubsection{Multi-shell diffusion acquisition and multi-orientation analysis}

Figure 3.11 shows diffusion-weighted volumes acquired using $\mathrm{k}_{\mathrm{T}}$-dSTEAM at $1000 \mu \mathrm{m}$ isotropic over a range of b-values in order to investigate the 
basic diffusion-induced signal decay and resulting image quality. High b-values could be achieved at reasonable SNR, up to $6000 \mathrm{~s} / \mathrm{mm}^{2}$ in the low- $\mathrm{T}_{2} /$ low-D specimen 1 (Figure 3.11B) and up to $8000 \mathrm{~s} / \mathrm{mm}^{2}$ in the high$\mathrm{T}_{2}$ /high-D in specimen 2. It is evident that for both specimens, SNR decreases as a function of $b$-value. At about $b=4000 \mathrm{~s} / \mathrm{mm}^{2}$, the higher unweighted signal but also higher diffusivity in GM compared to WM results in a loss of WM/GM contrast. For both specimens, the majority of diffusion-induced signal decay is observed between $b=2000 \mathrm{~s} / \mathrm{mm}^{2}$ and $b$ $=4000 \mathrm{~s} / \mathrm{mm}^{2}$. It must be noted that the increased signal decay at higher $\mathrm{b}$-values is not only intrinsic to the diffusion-weighting but it is also due to increasing $\mathrm{T}_{1}$-weighting in the STE signal, since higher $\mathrm{b}$-values are acquired with longer mixing times. Because $\mathrm{T}_{1}$ for specimen 1 also is lower than that of specimen 2, this explains the lower CNR in specimen 1 at increasing b-value in comparison to specimen 2 . However, the $T_{1}$ contribution in the signal decay does not affect the signal difference in different diffusion directions (Figure 3.9B, bottom). In the case of specimen 1 , where the mid-sagittal genu of the $\mathrm{CC}$ is taken as a reference, the signal decays faster along the LR $\left(b_{z}\right)$ direction in comparison with perpendicular AP $\left(b_{\mathrm{x}}\right)$ or superior-inferior $\left(\mathrm{SI}-\mathrm{b}_{\mathrm{y}}\right)$ directions, as expected.

Having established the quality of $\mathrm{k}_{\mathrm{T}}$-dSTEAM data over multiple b-values, we analysed both single-shell (using Equation 3.6) and multi-shell $\mathrm{k}_{\mathrm{T}}$-dSTEAM data using the B\&S $\mathrm{S}_{\mathrm{r} 2}$ model to investigate the support for the fitting of multiple fibre orientations in a voxel. Varying-TM multi-shell diffusion data analysis was performed using the $\mathrm{qT}_{1}$ map (Figure 3.10A) and Equation 3.5, following the pipeline shown in Figure 3.4. The comparison between fitting results using single-shell and multi-shell analysis is shown in Figure 3.12. Note that the $\mathrm{S}_{0} *$ fit for the multi-shell analysis is an extrapolated fit of the 'zero-TM' non $\mathrm{T}_{1}$-weighted signal derived from the $\mathrm{b}_{0}$ volumes at the different TM's (Figure 3.4), in contrast to the fits of $\mathrm{S}_{0}$ in the single-shell cases. As expected, the magnitude of extrapolated $\mathrm{S}_{0} *$ obtained in the multi-shell analysis is $\approx 10$ times higher than the b4k and b6 $\mathrm{k} \mathrm{S}_{0}$ (the colour scale is adjusted in Figure 3.12 for the multi-shell $\mathrm{S}_{0} *$ ). The multi-shell $\mathrm{S}_{0}$ * fit is more similar to the $\mathrm{b} 4 \mathrm{k} \mathrm{S}_{0}$ fit than to the $\mathrm{b} 6 \mathrm{k}$ $\mathrm{S}_{0}$ fit, lacking the signal drop near the genu of the CC visible in the $\mathrm{b} 6 \mathrm{k}$ $\mathrm{S}_{0}$ fit (indicated with the white arrow). This is also reflected in the stick fraction and orientation maps: whereas the b6k single-shell analysis suffers from a rather noisy estimation at the frontal location, the multi-shell analysis achieves reasonable estimates everywhere. Although the stick fraction resulting from the multi-shell fit is well defined, it has a slightly elevated level compared to the single-shell fractions. This is quantified 


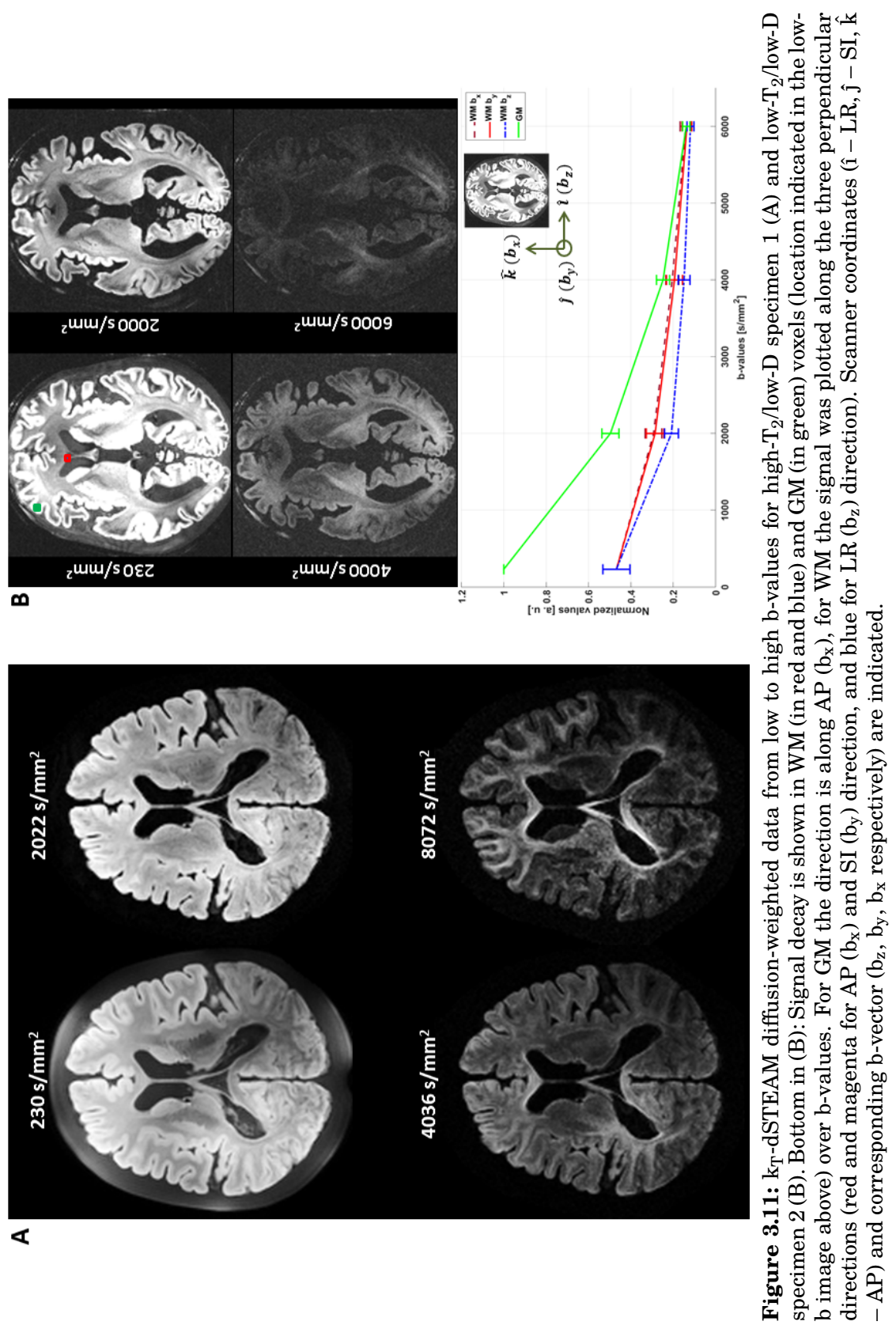


in Figure 3.12B, where the linear regression through scatter plots shows higher stick fraction values for the multi-shell analysis, leading to lower than unity regression slopes $\alpha$. This effect is stronger for b6k single-shell analysis $(\alpha=0.6941)$ than for b4k single-shell analysis $(\alpha=0.8409)$. However, the level differences in the stick fraction parameters do not seem to affect the orientation estimation, as shown in Figure 3.12A (bottom) and C. Comparing Stick $_{0}$ orientations in clearly defined tracts, such as the splenium and genu of the $\mathrm{CC}$ and the optical radiations (see yellow mask in Figure $3.10 \mathrm{C}$ ), $60 \%$ of the orientations are within $10^{\circ}$ and $80 \%$ of the orientations are within about $20^{\circ}$.

Next, we investigated the relative advantages of a varying-TM multi-shell acquisition and a same-TM multi-shell acquisition. To this end we acquired, for specimen 3 , a short TM b4k shell and long TM b6k shell, as before, and added a matched long TM b4k shell. As illustrated in supplementary figure 3.14 , there is a $10-15 \%$ decrease in SNR in going from the short TM to the long TM at b4k. However, this is relatively modest compared to the 25-30\% SNR decrease in going from short TM b4k to long TM b6k. Therefore, it may be justified in some cases to forego the SNR advantage (in the b4k shell) of a varying-TM multi-shell acquisition and instead choose a same-TM multi-shell acquisition. Ball \& Stick modelling $\left(B \& \mathrm{~S}_{\mathrm{r} 2}\right)$ of both the varying-TM and same-TM options on the same specimen shows that a high correlation in stick fraction $\left(\mathrm{R}^{2}=0.926\right)$ and highly similar fibre orientation fits are obtained, with $90 \%$ of single-tract orientations within $20^{\circ}$ (supplementary figure 3.15 ).

Figure 3.13 shows the comparison between single orientation and multiple orientation analysis of $\mathrm{k}_{\mathrm{T}}$-dSTEAM multi-shell data and the resulting tractography in the cerebellar peduncle. The use of multi-shell multiorientation tractography enables superior definition of crossing fibre structures, such as the raphe nuclei fibres (at the white arrow) and transverse pontine fibres (at the yellow arrow). This is true both in comparison to a multi-orientation analysis of the high-b single-shell data (Figure 3.13A, B) and in comparison to the single orientation analysis of the multi-shell data (Figure 3.13C, D). 


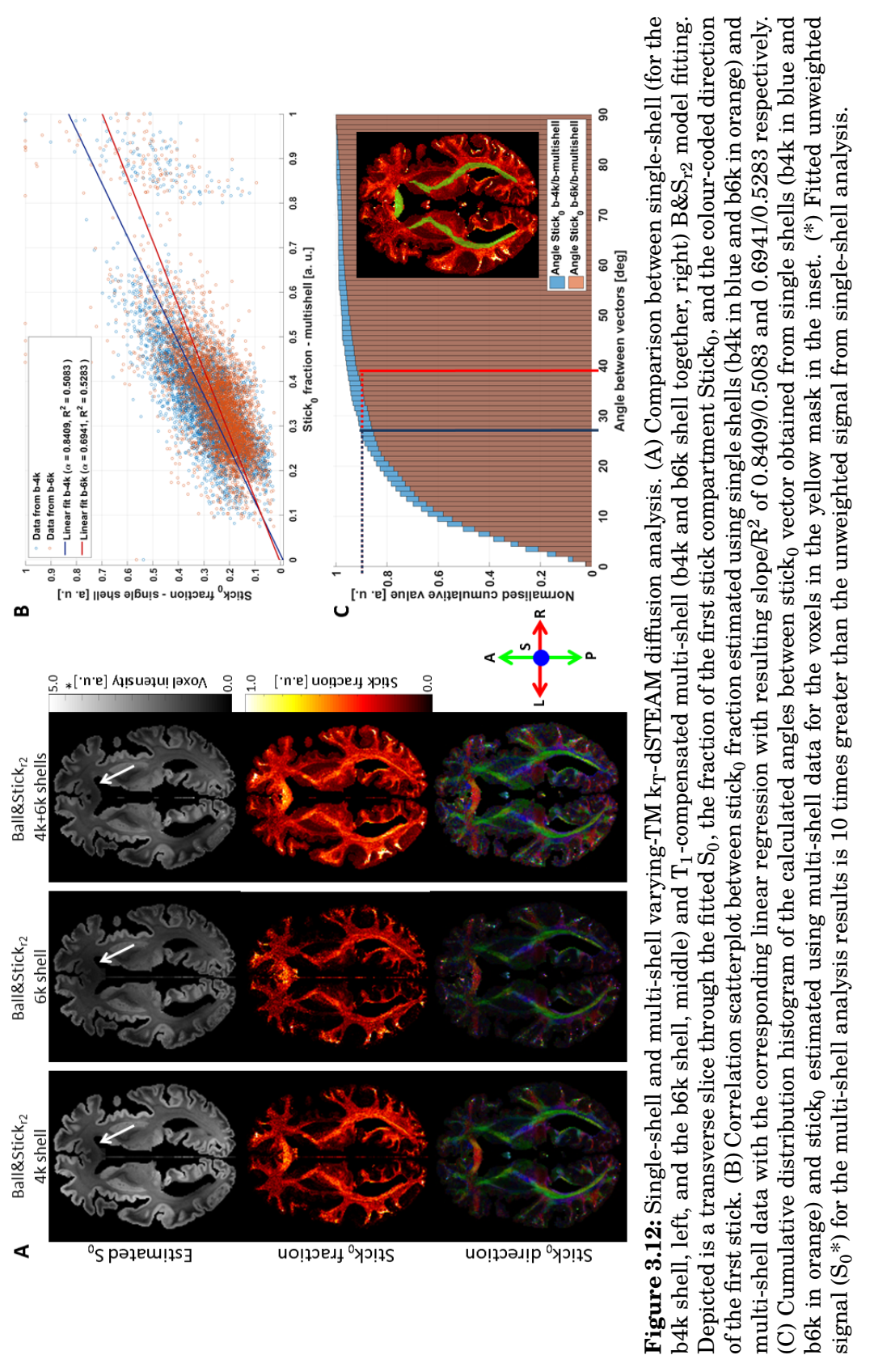




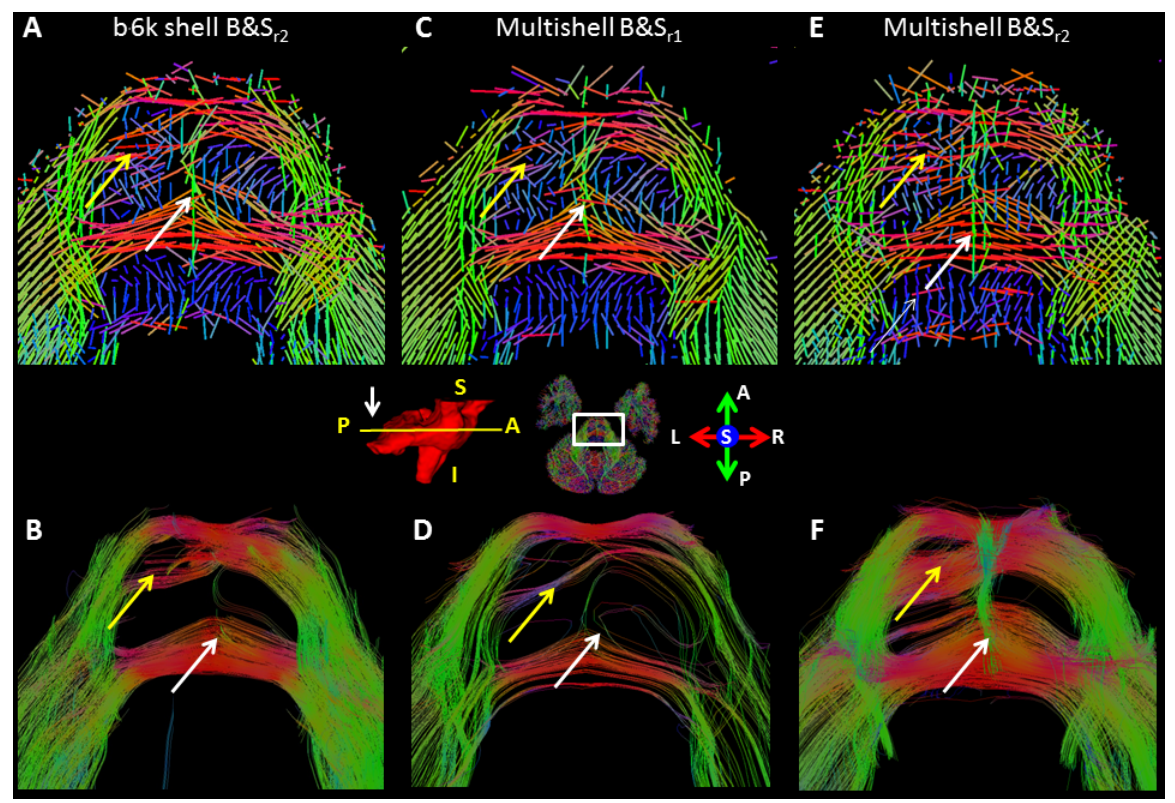

Figure 3.13: $\mathrm{k}_{\mathrm{T}}$-dSTEAM multi-shell multi-orientation fitting and tractography of the cerebellar peduncle. A, C and $\mathrm{E}$ show the orientations of sticks using: the $\mathrm{B} \& \mathrm{~S}_{\mathrm{r} 2}$ model for a single b6k shell (A), the B\&S $\mathrm{r}_{1}$ model for multi-shell (b4k + b6k) data (C) the B\& $\mathrm{S}_{\mathrm{r} 2}$ model for multi-shell $(\mathrm{b} 4 \mathrm{k}+\mathrm{b} 6 \mathrm{k})$ data $(\mathrm{E})$ at the position indicated in the inset. B, D and $\mathrm{F}$ show the corresponding tractography on the orientations in A, C and E, respectively. Arrows indicate the raphe nuclei projections (green streamlines at the white arrow) and transverse pontine fibres (red streamlines at the yellow arrow).

\subsection{Discussion}

We show that $\mathrm{k}_{\mathrm{T}}$-dSTEAM achieves homogeneous $\mathrm{T}_{1}, \mathrm{~T}_{2}$ and diffusionweighted STEAM signal across human whole-brain and hemisphere specimens at 9.4 $\mathrm{T}$, solving a complex $\mathrm{B}_{1}{ }^{+}$homogenisation problem for the threepulse STEAM situation (Figure 3.1) where current solutions do not suffice. As shown in Figure 3.2B, the use of a composite $\mathrm{k}_{\mathrm{T}}$-points pulse only for excitation, as performed in a single pulse sequence such as GRE, is not enough to improve the signal homogeneity. $\mathrm{k}_{\mathrm{T}}$-dSTEAM's full application of composite pulses in all the three STEAM pulses is the only way to achieve $\mathrm{B}_{1}{ }^{+}$homogeneity (Figure $3.2 \mathrm{C}$ ). Composite $\mathrm{k}_{\mathrm{T}}$-points pulses have been used in sequences with more than one pulse before, as for example in 
turbo spin-echo (TSE) sequences [72] but, to our knowledge, this is the first time they are used in a three pulse combination as is the case for STEAM. Solving the challenge of getting a more homogeneous signal over intact human brains allowed us to use the advantages of STEAM diffusion-weighting at UHF: creating a large part of diffusion contrast during $\mathrm{T}_{1}$ decay, which is more advantageous at higher fields for fixed post mortem tissue. Even though a stimulated echo has half the signal (before $T_{1}$ or $T_{2}$ decay) of a spin-echo from PGSE, in the whole ex vivo brain context of short $\mathrm{T}_{2}$ 's, high b-values and low gradient performance, this is compensated with the advantage of less $\mathrm{T}_{2}$ decay for a given $b$-value. This advantage is clearly illustrated in the $\mathrm{k}_{\mathrm{T}}$-PGSE vs. $\mathrm{k}_{\mathrm{T}}$-dSTEAM comparison (Figure 3.5 and 3.6 , showing a $20-40 \%$ SNR advantage for single diffusion-weighted volumes for b-values of 4000 to $6000 \mathrm{~s} / \mathrm{mm}^{2}$, resulting in increased precision (reduced noise and variability) and accuracy (reduced bias) in dMRI modelling results. Of course, this does not conclude that high precision and high accuracy results could not be obtained with PGSE in the investigation of large human brain specimen. For instance, approximately $40 \%$ of SNR could be regained by doubling PGSE acquisition time and obtaining two averages of each volume. However, the fundamentally higher SNRper-unit-time or SNR-efficiency of $\mathrm{k}_{\mathrm{T}}$-dSTEAM implies that $\mathrm{k}_{\mathrm{T}}$-dSTEAM could, in the same time, either boost SNR further by another $40 \%$ through averaging, or acquire twice the number of diffusion directions at the same SNR, making it a superior option. It should be noted that this conclusion is specific to the investigated case of human whole-brain or hemisphere samples which are formalin-fixed and investigated in large-bore scanners. Small tissue samples can be investigated in smaller bore systems with much higher performing gradients, often in excess of 5 times the maximum gradient amplitude $(80 \mathrm{mT} / \mathrm{m})$ available in this study. In the smallbore high max-G situation, $\mathrm{T}_{2}$ signal decay can be effectively mitigated by using very short gradient pulses and TE's, and high quality dMRI data can still be obtained for high b-values. However, in general, with higher field-strengths (and, hence, shorter $\mathrm{T}_{2}$ 's), lower gradient performance, and higher desired $b$-values and diffusion times, the advantages of STEAM compared to PGSE will increase. Finally, whole human brain ex vivo diffusion MRI also could be achieved by in-situ acquisition very soon after death, at which point relaxation parameters, such as $\mathrm{T}_{2}$ would be much closer to in vivo values. However, autolysis and tissue degeneration sets in almost immediately after death and progresses steadily in the hours thereafter. This means an in-situ ex vivo acquisition does not take place in a physiological steady-state condition. Moreover, acquisitions over sev- 
eral days, as performed for fixed brain samples, are therefore much more difficult.

In the future, further advantages can be achieved by utilising the fact that the storing pulse splits the magnetisation, where half the signal forms the first spin-echo after the storing pulse and another half of the signal is stored along the longitudinal axis and is available for a stimulated echo. Both the SE and STE can be phase encoded and read out without interference $[85,138]$, doubling the effective data rate. In the context of $\mathrm{k}_{\mathrm{T}^{-}}$ dSTEAM (Figure 3.2), this would mean moving the spoiler gradient (green in Figure 3.2C) towards the recalling pulse and adding a diffusion gradient and EPI train after the storing pulse, similar to those after the recalling pulse. One would then have both a diffusion-weighted SE and a diffusionweighted STE signal available in the same acquisition time, although the $\mathrm{b}$-value for the SE would always be considerably lower than that the STE. The practical use of both echoes in the same model fit necessitates further advances in modelling (including dealing with different diffusion- and $\mathrm{T}_{1}$ weighted echoes, along the lines set out in this work for STE only) and careful consideration of the steady-state conditions for the SE with corresponding spoiling and diffusion-gradient requirements.

\subsubsection{Ultra-high resolution whole-brain diffusion MRI}

We use the $\mathrm{k}_{\mathrm{T}}$-dSTEAM sequence to obtain high resolution diffusion-weighted data in ex vivo specimens. We achieve ultra-high isotropic resolution wholebrain dMRI data, $400 \mu \mathrm{m}$ for high $\mathrm{T}_{2}$ /high-D specimen 2 , which to our knowledge is the highest isotropic resolution reported for ex vivo wholebrain diffusion acquisition. There exists a clear trade-off in weighing higher diffusion-weighting against higher resolution (at the same SNR). $\mathrm{k}_{\mathrm{T}}$-dSTEAM acquisitions at very high b-values (over $5000 \mathrm{~s} / \mathrm{mm}^{2}$ ) can be achieved in low $\mathrm{T}_{2}$ /low-D ex vivo samples and even higher b-values (over $8000 \mathrm{~s} / \mathrm{mm}^{2}$ ) in high $\mathrm{T}_{2}$ /high-D samples (Figure 3.10) at $1000 \mu \mathrm{m}$ isotropic. Volumes with an $8 \mathrm{x}$ higher $(500 \mu \mathrm{m})$, and even $16 \mathrm{x}$ higher $(400 \mu \mathrm{m})$ resolution could be acquired at lower b-values $\left(3000-4000 \mathrm{~s} / \mathrm{mm}^{2}\right)$ as shown in Figure 3.7. These ultra-high resolution acquisitions $(400-500 \mu \mathrm{m})$ were calibrated to have enough SNR to be submitted to diffusion imaging and tractography pipelines (Figure 3.8,3.9) and show that the $\mathrm{k}_{\mathrm{T}}$-dSTEAM sequence has enough SNR efficiency to produce useful data in this SNR challenged regime. It should be noted that the achievable resolution (at a given SNR and b-values) or the achievable b-value (at a given SNR and resolution) also 
depends on the quality of the sample, notably its $T_{2}$ value. Post mortem acquisitions, in particular on large human samples which are scarce, need to be individually tailored to the quality of the sample, which explains our choices for differences in acquisitions, e.g. an $\approx 2 \mathrm{x}$ higher resolution for specimen $2\left(400 \mu \mathrm{m}\right.$ with a $\mathrm{T}_{2}$ of $\left.18 \mathrm{~ms}\right)$ than for specimen $1(500 \mu \mathrm{m}$ with a $\mathrm{T}_{2}$ of $9 \mathrm{~ms}$ ). As an illustration of the advantages of the higher resolution, tractography across the WM/GM border shows less of the unwanted gyral crown bias, and more high-curvature paths connecting the sulcal wall. Simple deterministic streamlining tractography was used to emphasise the effect of data resolution, rather than the effect of tractography technique. More sophisticated tractography could support high-curvature paths into the sulcal wall in lower resolution data, possibly with additional assumptions on likely insertion paths. Future combination of sophisticated tractography and the ultra-high resolution whole-brain diffusion MRI data presented here could improve the fidelity of MRI-based human cortico-cortical connectomes.

\subsubsection{Relaxometry for quantitative $T_{1}$ and $T_{2}$ mapping}

Quantitative $T_{1}$ and $T_{2}$ could be estimated by using $T_{1} w$ and $T_{2} \mathrm{w}_{\mathrm{T}^{-}}$ dSTEAM acquisitions by varying TM and TE respectively. In the case of the $\mathrm{qT}_{1}$ relaxometry, Equation 3.2 can be used in short TR conditions. This short TR equation is also used in the multi-shell diffusion analysis pipeline. The estimated $\mathrm{S}_{0} *$ in $\mathrm{qT}_{1}$ relaxometry contains information about the proton density, coil sensitivity and $\mathrm{T}_{2}$ with high contrast between WM and GM in $\mathrm{S}_{0} *$ maps. The resulting $\mathrm{qT}_{1}$ values for $\mathrm{WM}$ and GM are approximately in a range between 0.2 and $0.7 \mathrm{sec}$ with no clear contrast between WM and GM. In contrast with the $\mathrm{qT}_{1}$ estimation, the $\mathrm{qT}_{2}$ map and corresponding histogram show a well-defined contrast between WM and GM structures in the specimen. The estimated $\mathrm{S}_{0}$ for $\mathrm{qT}_{2}$ modelling shows little contrast between WM and GM, containing information about the proton density, coil sensitivity, and $\mathrm{T}_{1}$. This is in agreement with observations that $T_{2} / T_{2}{ }^{*}$, rather than $T_{1}$, dominates the contrast in ex vivo images [14].

\subsubsection{Multi-shell diffusion acquisition and multi-orientation analysis}

Multi-shell analysis of $\mathrm{k}_{\mathrm{T}}$-dSTEAM at variable TM (varying-TM) is shown as an approach for ex vivo data analysis which is adapted to the strengths 
of STEAM diffusion-weighting. The varying-TM analysis incorporates $\mathrm{qT}_{1}$ parameter maps and uses the cascaded modelling pipeline in Figure 3.4, which is based on the signal model in Equation 3.5. This allows parameter estimates, such as stick volume fractions and orientations, for varyingTM data which are not well suited for analysis with standard models. The advantage of acquiring varying-TM data is that SNR at each shell is maximised, rather than compromising SNR for lower b shells (c.f Supplementary Figure 3.14). Therefore, the high SNR of low-b shells and the high orientation contrast of high-b shells are used effectively. Here, we used this gain for multi-orientation modelling and crossing-fibre tractography. An additional advantage of varying-TM multi-shell acquisitions is that they could support sophisticated diffusion microstructure modelling [6] which relies on data with a range of different diffusion times, such as axon diameter modelling [5, 12, 15, 65, 70, 235]. However, future varyingTM microstructure modelling would have to take the decreasing apparent diffusion coefficient (ADC) with increasing diffusion time into account $[65,112,122]$, as well as the microstructural changes due to autolysis after death $[69,71,175]$. Equation 3.5 currently does not contain TM-dependent $\mathrm{ADC}$, which may explain the constant slope difference between stick fractions estimated from the single-shell and varying-TM multi-shell data (c.f. Figure 3.12B). It is important to note that the orientation modelling, which is the focus here, is not affected by any ADC-weighting in the stick fraction as clearly shown in Figure 3.13. The use of varying-TM multi-shell analysis is well suited for multi-orientation models as $B \& \mathrm{~S}_{\mathrm{r} 2}$ and $\mathrm{B} \& \mathrm{~S}_{\mathrm{r} 3}$ since it enables the incorporation of high SNR dMRI volumes at different diffusion directions and at different b-values. This supports better modelling of crossing fibres such as in the brain stem (Figure 3.13), by effectively using both low-b shells and high-b shells at the highest possible SNR and contrast. Future applications which aim at diffusion microstructure modelling of multi-shell data without the need for varying diffusion times, could employ the same-TM multi-shell acquisition (cf. Supplementary figure 3.15) without potentially introducing bias due to the varying-TM.

\subsubsection{Outlook}

In this work, $\mathrm{k}_{\mathrm{T}}$-dSTEAM data was acquired at 400-500 $\mu \mathrm{m}$ resolution with moderate $3000-4000 \mathrm{~s} / \mathrm{mm}^{2} \mathrm{~b}$-values single-shell and at $1000 \mu \mathrm{m}$ resolution with high $6000-8000 \mathrm{~s} / \mathrm{mm}^{2}$ b-values. A combination of ultra-high resolution and high b-values in the same dataset would have the com- 
bined advantage for tractography of delineating both high curvature fibres (by high resolution) and crossing fibres (by multi-shell multi-orientation modelling). However, under the current conditions, this would tax the SNR in the resulting data (or the acquisition times) too much. Nevertheless, such data could be acquired in the future by combining $\mathrm{k}_{\mathrm{T}}$-dSTEAM with one or more additional techniques. On the acquisition side, k-space undersampled acquisitions with parallel imaging reconstruction, as used in the relaxometry data here, can help balance the trade-off between a number of diffusion direction volumes, amount of time per volume and SNR. Denoising approaches that use the redundancy present in many diffusion volumes can help in regaining SNR in SNR-starved acquisitions $[24,189,215]$. Finally, it has been shown that high resolution/low-b data and low-resolution/high-b data in the same specimen can be combined in the analysis to provide the advantage of both $[78,186]$. Such ultra-high resolution and multi-shell $\mathrm{k}_{\mathrm{T}}$-dSTEAM data have the potential to improve the fidelity of MRI-based human cortico-cortical connectomes.

\subsection{Acknowledgement}

We thank Andreas Herrler for carefully selecting and fixing specimen 1 and making it available for this study. We also thank Katrin Amunts, Svenja Caspers and Karl Zilles for carefully selecting and fixing specimen 2 and making it available for this study. We also thank Roxana Kooijmans for carefully selecting and fixing specimen 3 and making it available for this study. AR and FJ were supported by an ERC Starting Grant (MULTICONNECT, \#639938). AR was further supported by a Dutch science foundation (NWO) VIDI Grant (\#14637). BP is partially funded by NWO VIDI Grant 016.Vidi.178.052 and NIH Brain Initiative (R01 MH111444; PI Feinberg). 


\subsection{Supplementary material}

A

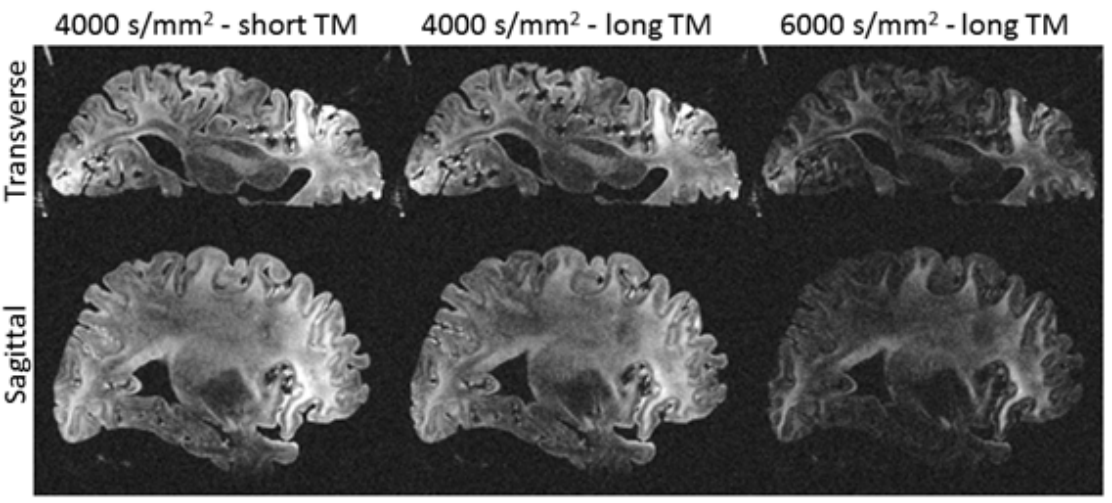

B

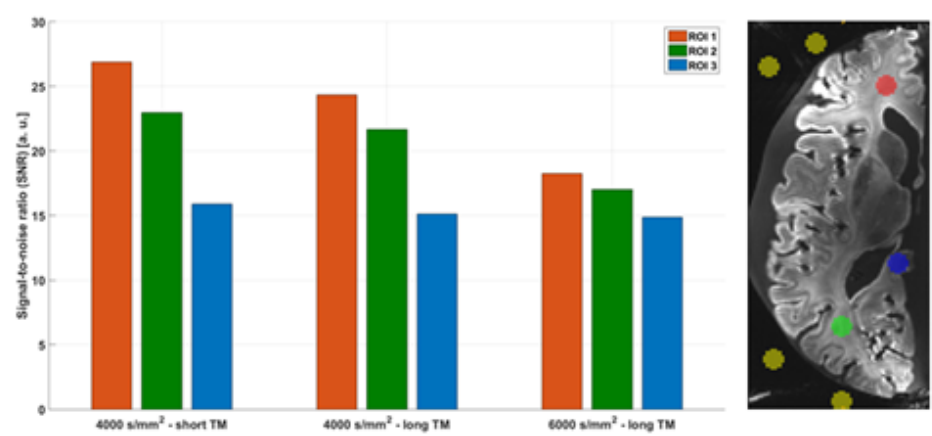

Figure 3.14: $\mathrm{k}_{\mathrm{T}}$-dSTEAM signal and SNR comparison for long and short TM. (A) Transverse (top) and sagittal (bottom) views for single diffusion-weighted volumes of specimen 3 acquired at $\mathrm{b}=4000 \mathrm{~s} / \mathrm{mm}^{2}$ (b4k) at TM's of $0.134 \mathrm{~s}$ (short) and $0.205 \mathrm{~s}$ (long) and, for comparison, at $\mathrm{b}=$ $6000 \mathrm{~s} / \mathrm{mm}^{2}$ (b6k) at long TM. (B) SNR was calculated in three different areas (red, green and blue ROI's in the inset, with noise evaluated in the yellow ROI's) over different b-values and TM's. The b4k/short TM and b6k/long TM data are combined in the multi-shell varying-TM analyses, whereas the b4k/long TM and b6k/long TM data are combined in the multi-shell same-TM analyses. 
A

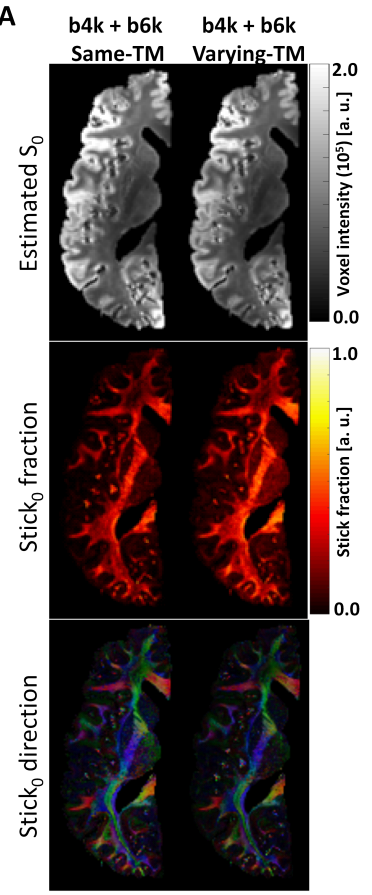

B

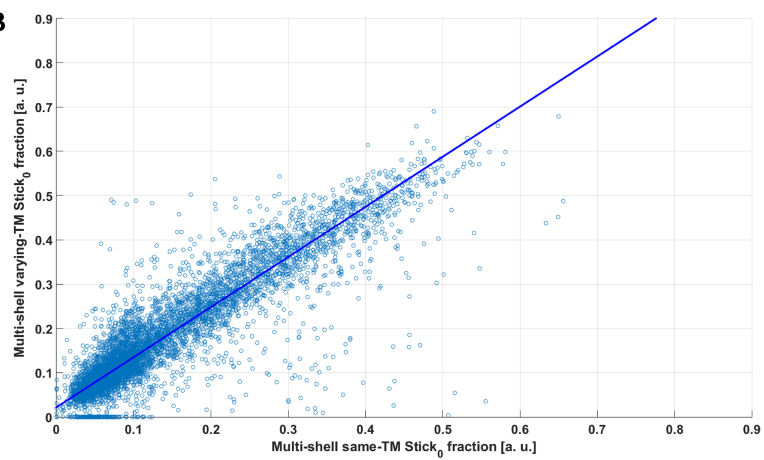

C

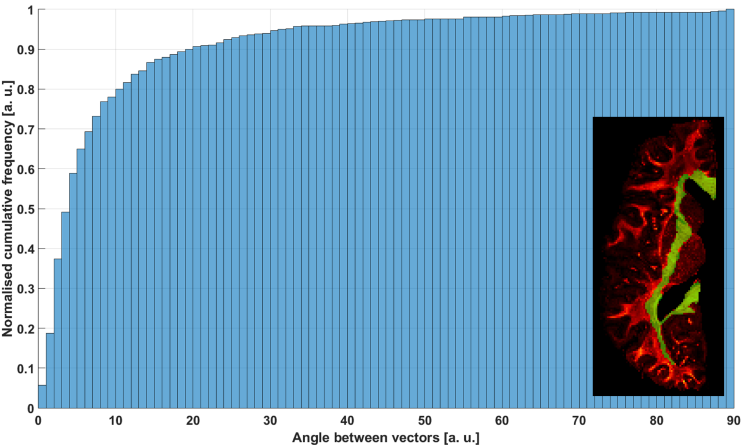

Figure 3.15: $\mathrm{k}_{\mathrm{T}}$-dSTEAM diffusion analysis comparison between multi-shell varying-TM modelling and multi-shell same-TM modelling. (A) Comparison between multi-shell sameTM analysis (right) and $\mathrm{T}_{1}$-compensated multi-shell varying-TM analysis (left). For sameTM B\& $\mathrm{S}_{\mathrm{r} 2}$ model fitting is performed as for single-shell analysis, whereas for varying-TM model fitting the pipeline in Figure 3.4 is used. Depicted is a transverse slice through the fitted $S_{0}$, fraction of the first stick compartment Stick $_{0} . \mathrm{w}$, and the colour-coded direction of

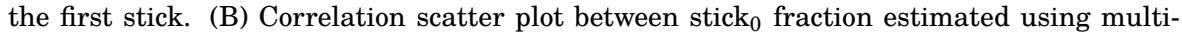
shell same-TM and varying-TM data with the corresponding linear regression (slope $/ \mathrm{R}^{2}$ of 1.1132/0.9263). (C) Cumulative distribution histogram of the calculated angles between stick $_{0}$ vectors obtained from multi-shell same-TM and multi-shell varying-TM data for the voxels in the yellow mask in the inset. $(*)$ Fitted unweighted signal $\left(\mathrm{S}_{0} *\right)$ for the multi-shell varying-TM analysis results is 10 times greater than the unweighted signal from the multishell same-TM analysis. 



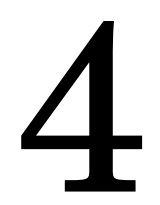

\section{Human whole-brain diffusion MRI at $450 \mu \mathrm{m}$ post mortem with $\mathrm{k}_{\mathrm{T}}$-dSSFP and a specialised $9.4 \mathrm{~T}$ RF-coil ${ }^{1}$}

\footnotetext{
${ }^{1}$ Adapted from the accepted abstracts: "(Lagos) Fritz et al. Whole human brain diffusion MRI at $450 \mu \mathrm{m}$ post mortem with dwSSFP and a specialized 9.4T RF-coil. ISMRM 24th Annual Meeting and Exhibition, 2016" (as Power Pitch), "Fritz et al. Whole human brain diffusion MRI at $450 \mu \mathrm{m}$ post mortem with dwSSFP and a specialized 9.4T RFcoil. ISMRM Benelux Chapter 8th Annual Meeting and Exhibition, Eindhoven, 2016" (accepted as Oral Presentation)
} 


\subsection{Introduction}

Diffusion-weighted MRI (dMRI) in ex vivo human brain specimens is a valuable tool for neuroanatomical investigations and it helps in the validation of white matter orientation estimates [129, 178], microstructure models $[12,129,150]$ and tractography $[173,179]$. It is also highly useful for the atlasing and mapping of human subcortical structures [2, 67] and the delineation of grey matter layers [3, 22, 121, 131]. The benefits of this technique emerge at high $(\approx 1000 \mu \mathrm{m}$ isotropic $)$ and far higher resolutions $(\approx 400 \mu \mathrm{m}$ isotropic) [60]. However, increasing image resolution for ex vivo diffusion imaging carries several difficulties primarily because of two effects: strongly reduced diffusivity (at least half) and $\mathrm{T}_{2}[163,59,175]$. Therefore, dMRI sequences applied in this context must be capable of acquiring dMRI data at higher b-values to compensate this reduced diffusivity, at least double commonly used in vivo values [59, 147]. At the same time, it must achieve a lower echo time (TE) than values suitable for in vivo use to avoid excessive signal loss through the fast $\mathrm{T}_{2}$ signal decay. For the pulsed gradient spin-echo (PGSE) sequence, which is the most common dMRI pulse sequence, it is very challenging to meet these requirements. This is because of the intrinsically intertwined relationship between b-value and TE, in which higher b-values require longer TE's, which then leads to stronger signal reduction due to $\mathrm{T}_{2}$ decay. Nevertheless, it is still possible to acquire high-quality ex vivo dMRI data with the PGSE sequence on small-bore preclinical or animal MRI systems (e.g. see references $[3,173,179,42,79,92,151,210])$. In that case, the superior gradient performance (which allows shorter TE thanks to reduced diffusion gradient and EPI readout duration) and long scanning sessions (many hours to multiple days, which allows averaging) yield ultra-high resolution datasets far below the millimetre scale while maintaining a sufficiently high SNR for the acquired images. However, the drawback of small-bore systems is limited tissue sample size (often less than about $20 \times 20 \times 20 \mathrm{~mm}^{3}$, and generally smaller than about $50 \times 50 \times 50 \mathrm{~mm}^{3}$ ), severely restricting the size of the brain region examined. This is a strong practical limitation for the use on human brain samples, since even single brain areas in the human brain can exceed this size.

If the goal of Neuroscience is to provide the important context of entire white matter (WM) network and entire grey matter areas connected through these systems, the acquisition of ex vivo dMRI for intact human brain samples necessarily has to take place in large-bore systems. This re-introduces 
the challenge of limited gradient performance compared to small-bore systems, and the relative inefficiency of PGSE already mentioned.

In this context, it is necessary to explore the use of other dMRI sequences that can be employed for high resolution and high diffusion-weighting. One of those is the diffusion-weighted Steady-State Free Precession (dSSFP) sequence. Earlier works by Miller et al. 2012 [147] and Foxley et al. 2014 [84] have shown whole-brain ex vivo dMRI SSFP data at $3 \mathrm{~T}$ and $7 \mathrm{~T}$, respectively, and demonstrated that this sequence can achieve much higher SNR efficiency than PGSE for that purpose. Additionally, increasing the main magnetic field strength $\left(B_{0}\right)$ resulted in a further increased SNR efficiency and contrast-to-noise ratio (CNR) in ex vivo dMRI. Therefore, using $\mathrm{dSSPF}$ at even higher $\mathrm{B}_{0}$ (i.e. 9.4 $\mathrm{T}$ ) appears promising for high quality dMRI data.

One drawback of using ultra-high field scanners (UHF, $\mathrm{B}_{0}>=7 \mathrm{~T}$ ) is the increase of the radiofrequency $(\mathrm{RF})$ field inhomogeneity, i.e. $\mathrm{B}_{1}{ }^{+}$inhomogeneity (see references [125, 211]), negatively affecting the contrast and signal homogeneity of the acquired data. For instance, at $9.4 \mathrm{~T}$, the required RF excitation wavelength is approximately $8 \mathrm{~cm}$ (in brain tissue this wavelength can be even shorter due to its relative permittivity) which leads to destructive interferences along a whole-brain specimen. One approach to compensate the $\mathrm{B}_{1}{ }^{+}$inhomogeneity is by using parallel RF transmission (pTx, [181]). pTx is a system where the RF coil doesn't transmit a unique $\mathrm{B}_{1}{ }^{+}$field (i.e. by a circularly polarised (CP) phase distribution according to the transmit channels coil position) but, instead, a set of $\mathrm{B}_{1}{ }^{+}$ subfields per element (i.e. a coil's channels are each driven by a unique RF pulse). The combination of those subfields generates a more homogeneous $\mathrm{B}_{1}{ }^{+}$field. In gradient recalled echo (GRE) sequences, the non-selective rectangular excitation pulse was replaced by a composite pulse created using the $\mathrm{k}_{\mathrm{T}}$-points method [53]. This method has already been applied in [180] for post mortem acquisitions on tissue sizes of approximately $80 \mathrm{x}$ $80 \times 80 \mathrm{~mm}^{3}$. Therefore, given the sequence similarity between GRE and SSFP, we asked whether the $\mathrm{k}_{\mathrm{T}}$-points $\mathrm{B}_{1}{ }^{+}$homogenisation method can be used in a similar manner for the dSSFP sequence.

In this work, we aimed to achieve high resolution with high SNR and CNR in whole-brain human specimen dMRI by using the dSSFP sequence on a 9.4 T human bore MR scanner. Consequently, we need to address the following objectives: First, we have to achieve $\mathrm{B}_{1}{ }^{+}$homogenisation across whole human brain specimens on a $9.4 \mathrm{~T} \mathrm{MR}$ system by integrating the $\mathrm{k}_{\mathrm{T}^{-}}$ points technique in the dSSFP sequence (hereafter named $\mathrm{k}_{\mathrm{T}}$-dSSFP). Second, we use the $\mathrm{k}_{\mathrm{T}}$-dSSFP sequence to obtain multi-shell moderate b-value 
diffusion-weighted data $\left(4000 \mathrm{~s} / \mathrm{mm}^{2}\right)$ at ultra-high whole post mortem brain resolution $(500 \mu \mathrm{m}$ to $450 \mu \mathrm{m})$. Finally, we perform diffusion tensor imaging (SE-DTI) analysis in the $\mathrm{k}_{\mathrm{T}}$-dSSFP data at both resolutions. We enable high time-efficiency in ultra-high resolution diffusion imaging of the whole human brain. We illustrate how ultra-high resolution (450 $\mu \mathrm{m}$ isotropic) whole-brain data shows a very good contrast and delineation between different tissues (white/grey matter) and between different tracts in white matter.

\subsection{Methods}

Two human brain hemispheres from two different subjects were used in the present study. One hemisphere was obtained from a subject without neurological or psychiatric diseases, while the second hemisphere was obtained from a patient with Alzheimer's Disease. The hemispheres were enclosed in a conformal container whose dimensions are suitable for human brains (Figure 4.1A). It was inserted into the custom-designed $9.4 \mathrm{~T}$ 8-channel pTx, $24 \mathrm{Ch}$ receive $\mathrm{RF}$-coil built onto a conformal receive former modelled as a precise fit around the container (Figure 4.1D and E).
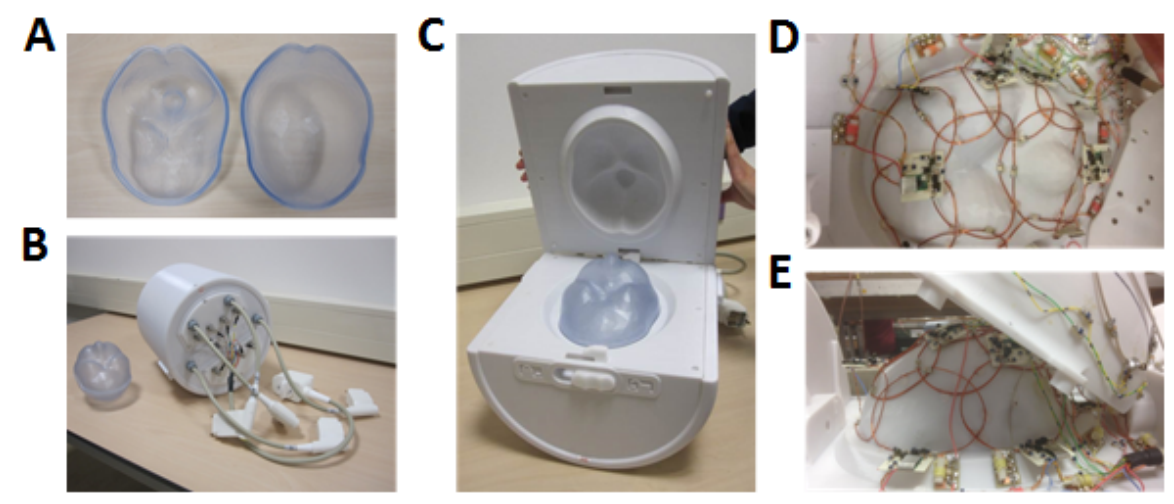

Figure 4.1: A) The conformal whole-brain container with opened inferior (left) and superior (right) halves. B) The entire assembled RF-coil array closed, viewed from the back, C) The closed container in the opened RF-coil array, showing conformal matching geometry of container and inner receive array former. D and E) View into the disassembled upper (D) and lower (E) casing.

Experiments were performed on a $9.4 \mathrm{~T} 82 \mathrm{~cm}$ bore human MR scanner 
(Magnetom 9.4 T, Siemens Medical Solutions, Erlangen, Germany) with an $80 \mathrm{mT} / \mathrm{m}, 330 \mathrm{~T} / \mathrm{m} / \mathrm{s}$ head gradient system, using 8-channels of its 16channel pTx system ( $1 \mathrm{KW}$ per channel). Prior to acquiring high resolution diffusion-weighted data (500 and $450 \mu \mathrm{m}$ isotropic, Table 4.1 ), $\mathrm{B}_{0}$ shimming and $\mathrm{B}_{1}{ }^{+} \mathrm{pTx}$ pulse calculations were performed. First, a localizer was acquired for spatial-localisation reference; then a $B_{0}$ fieldmap [57] and a $\mathrm{T}_{2}$ and $\mathrm{T}_{2} *$ compensated version of DREAM [152] were acquired to characterise $\mathrm{B}_{0}$ and $\mathrm{B}_{1}{ }^{+}$profiles in the specimen. An in-house routine in MATLAB (MathWorks, MA, USA) used those acquisitions to optimise both $\mathrm{B}_{0}$ shimming and $\mathrm{B}_{1}{ }^{+}$pulse calculation [181]. $\mathrm{B}_{0}$ shimming was performed with two to three iterations to improve $\mathrm{B}_{0}$ homogeneity. For the $\mathrm{k}_{\mathrm{T}^{-}}$ points pulse design [53], the resulting $\mathrm{B}_{0}$ and $\mathrm{B}_{1}{ }^{+}$maps after $\mathrm{B}_{0}$ shimming were used and composite pulses were calculated using the magnitude least square (MLS) approach [208] (c.f. Chapter 3). $\mathrm{k}_{\mathrm{T}}$-points sub-pulse spacing was set to $280 \mu \mathrm{s}$ for the excitation composite pulse as shown in Figure 4.2 .

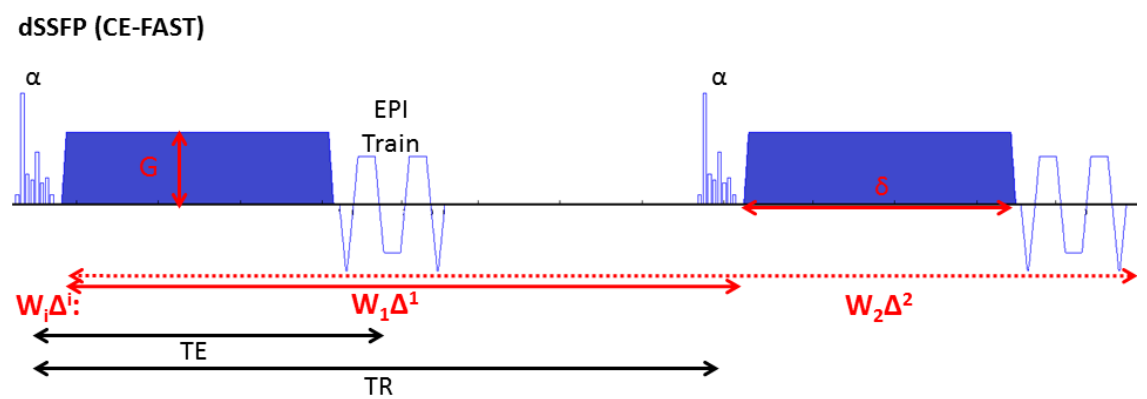

Figure 4.2: $\mathrm{k}_{\mathrm{T}}$-dSSFP sequence diagram: the rectangular excitation RF pulse was replaced by a composite $\mathrm{k}_{\mathrm{T}}$-points pulse to achieve higher $\mathrm{B}_{1}{ }^{+}$homogeneity at $9.4 \mathrm{~T}$. Diffusion parameters gradient amplitude $(G)$ and duration $(\delta)$, and diffusion time $(\Delta)$ per each repetition time (defined by the index i) are indicated. The total diffusion contrast is resulted by an accumulation (estimated from the weighted (defined by $\omega_{\mathrm{i}}$ ) sum, in Einstein notation.) of the diffusion times $\left(\Delta^{\mathrm{i}}\right)$ across all TR.

Subsequently, a set of 3D diffusion-weighted $\mathrm{k}_{\mathrm{T}}$-dSSFP volumes at 500 and $450 \mu \mathrm{m}$ isotropic resolution were acquired (as specified in Table 4.1). For all acquisitions, image resolutions and coverage (the field of view $(\mathrm{FoV})$ of $162 \times 150 \mathrm{~mm}^{2}$ in sagittal orientation) were kept fixed. In SSFP sequences, in contrast to PGSE sequences, the diffusion-weighting parameter under direct control is the q-value (in units of (length) ${ }^{-1}$ and defined as $q$ 
$=(2 \pi)^{-1} \gamma \delta \mathrm{G}$, with $\gamma$ the gyromagnetic ratio), rather than the $b$-value, which is not well defined for dSSFP. To calculate the effective b-value, which is reported for the $\mathrm{k}_{\mathrm{T}}$-dSSFP sequence, the ratio between the SSFP signal $\left(\mathrm{S}_{\mathrm{dSSFP}}\right.$ ) at a specific $\mathrm{q}$-value (with defined $\mathrm{T}_{1}, \mathrm{~T}_{2}$ and $\mathrm{B}_{1}{ }^{+}$values) and at $\mathrm{q}$ $=0$ (often in units of $\left.(\mathrm{cm})^{-1}\right)$ is taken. This signal equation is defined as:

$$
\begin{array}{r}
S_{d S S F P}: \frac{-M_{0}\left(1-E_{1}\right) E_{2} A_{2}^{-2 / 3}\left(F_{1}-E_{2} A_{1} A_{2}^{2 / 3}\right) \sin (\alpha)}{r-F_{1} s} \text { where } \\
F_{1}: K-\sqrt{K^{2}-A_{2}^{2}}, K: \frac{1-E_{1} A_{1} \cos (\alpha)-E_{2}^{2} A_{1}^{2} A_{2}^{-2 / 3}\left(E_{1} A_{1}-\cos (\alpha)\right.}{E_{2} A_{1} A_{2}^{-4 / 3}(1+\cos (\alpha))\left(1-E_{1} A_{1}\right)} \\
r: 1-E_{1} \cos (\alpha)+E_{2}^{2} A_{1} A_{2}^{1 / 3}\left(\cos (\alpha)-E_{1}\right) \\
s: E_{2} A_{1} A_{2}^{-4 / 3}\left(1-E_{1} \cos (\alpha)\right)+E_{2} A_{2}^{-1 / 3}(\cos \alpha-1) \\
A_{1}: \exp (-b D), \quad A_{2}: \exp (-\beta D), \quad b:(\gamma G \delta)^{2} T R, \quad \beta:(\gamma G \delta)^{2} \delta, \\
E_{1}: \exp \left(-T R / T_{1}\right) \quad \text { and } \quad E_{2}: \exp \left(-T R / T_{2}\right)
\end{array}
$$

(from Appendix 1 section (A1) in the work of McNab et al. 2008 [143] and in the Introduction chapter of this thesis, Equation 2.22). In this study, the effective $b$-value was estimated for white matter $\left(\mathrm{T}_{1} \approx 400 \mathrm{~ms}\right.$ and $\mathrm{T}_{2} \approx 10$ $\mathrm{ms}$ ) and used for diffusion analysis. Given the complexity of the SSFP signal equation, several parameters were optimised to achieve optimal SNR and contrast-to-noise ratio (CNR) for a given b-value, as suggested in the work of Miller et al. 2012 [147]. In this optimisation constraints like maximum gradient amplitude, duty cycle and heat-load of the $9.4 \mathrm{~T}$ gradient system were considered. For comparison, a heat-load parameter was estimated by approximating the total work load (i.e. equal to the total gradient moment, mainly from the diffusion gradients) of the gradients during TR. The estimated heat-load for all the acquisitions were normalised with respect to the acquisition performed at $7 \mathrm{~T}$ [84] (Table 4.1). Reconstruction was performed using root of sum-of-squares (rSos) for full-sampled $3 \mathrm{D} \mathrm{k}$-space. $\mathrm{k}_{\mathrm{T}}$-dSSFP data were analysed using the PGSE-based diffusion tensor imaging (SE-DTI) model in FSL v4.0.1 [110]. This analysis was performed by using multiple diffusion shells: the lowest (which is considered as $a b_{0}$ reference volume) and the moderate or high q-values (which approximates to an effective b-value of $2000 \mathrm{~s} / \mathrm{mm}^{2}$ ) per resolution ${ }^{2}$, given

${ }^{2}$ SE-DTI requires, at minimum, $a \mathrm{~b}_{0}$ and b-value of $1000 \mathrm{~s} / \mathrm{mm}^{2}$ for in vivo diffusivity values 
the assumption of Gaussian diffusivity.

\begin{tabular}{|c|c|c|c|}
\hline Setup & $7 \mathrm{~T}$ & $9.4 \mathrm{~T}$ & $9.4 \mathrm{~T}$ \\
\hline${\text { Max q-value }\left(\mathrm{cm}^{-1}\right)}^{-1}$ & 300 & 300 & 300 \\
Coil (Tx/Rx) & $1 / 32$ & $8 / 24$ & $8 / 24$ \\
$\mathrm{~b}_{\text {eff }}\left(\mathrm{s} / \mathrm{mm}^{2}\right)$ & 5150 & 3682.2 & 4000 \\
TE/TR $(\mathrm{s})$ & $21 / 30$ & $25 / 36$ & $18 / 28$ \\
Resolution $(\mu \mathrm{m})$ & 1000 & 500 & 450 \\
EPI-factor & 3 & 3 & 1 \\
Max $\mathrm{G}_{\text {amp }}(\mathrm{mT} / \mathrm{m})$ & 56 & 50 & 75 \\
G-diffusion time $(\mathrm{ms})$ & 13 & 14.1 & 9.4 \\
Number directions & 49 & 72 & 66 \\
AT per volume (min:sec) & $11: 27$ & $19: 45$ & $40: 18$ \\
Relative heat-load & 1 & 0.72 & 1.39 \\
\hline
\end{tabular}

Table 4.1: $\mathrm{k}_{\mathrm{T}}$-dSSFP sequence parameters: Comparison of the sequence parameters used for the two ultra-high resolutions and the one reported in the work of Foxley et al. 2014 [84]. The heat-load is relative to the experiment performed in the $7 \mathrm{~T}$, where it quantifies the scanner's hardware workload.

\subsection{Results}

Figure 4.3 shows the transmit $\mathrm{B}_{1}{ }^{+}$maps for the standard non-selective and pTx transmit modes of the coil (Figure 4.3A top and bottom, respectively). Standard non-selective transmit mode shows large bands of (near) signal drop-out that are translated to dark bands in the dSSFP results (Figure 4.3B, top). Homogenised $\mathrm{B}_{1}{ }^{+}$by the $\mathrm{k}_{\mathrm{T}}$-points technique vastly improves the transmit profile (Figure 4.3A, bottom) and achieves homogeneous and higher signal, as well as high contrast (Figure 4.3B, bottom).

Figure 4.4 shows single volumes of whole-brain $\mathrm{k}_{\mathrm{T}}$ - $\mathrm{dSSFP}$ acquisitions for two ultra-high resolutions ( $500 \mu \mathrm{m}$ and $450 \mu \mathrm{m}$ ) at three different q-values with their respective effective b-value for white matter (WM). In the low-q acquisitions (for both resolutions) show homogeneous high-SNR $\mathrm{k}_{\mathrm{T}}$-SSFP signal. However, the increasing tissue contrast achieved at increasing qvalue is highly dependent on their respective $T_{1}, T_{2}$ and the excitation flip angle. Finally, at high-q value the highest resolution possesses better SNR, which can be explained due to better optimised sequence parameters at a higher gradient heat-load. Nevertheless, both sets of dMRI images

and at least, twice that for ex vivo [2] 


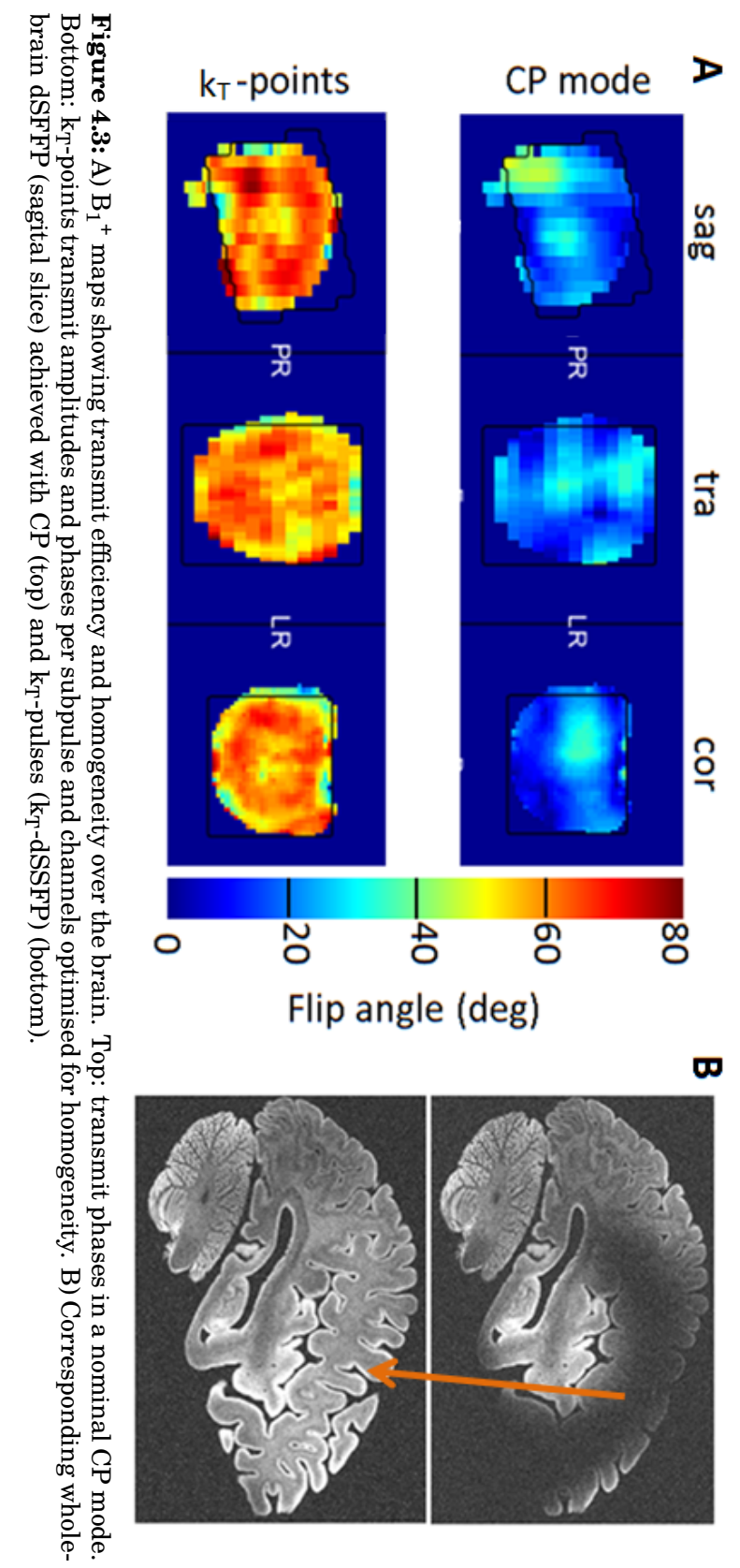


have sufficient SNR for diffusion analysis.

Figure 4.5 shows the SE-DTI analysis results from the acquired dMRI data at 500 and $450 \mu \mathrm{m}$ isotropic resolution. The corresponding fractional anisotropy (FA) and mean diffusivity (MD) maps show a high contrast between grey matter (GM) and WM, with well defined WM tracts in the FA maps, such as the corpus callosum and the optical tract (Figure 4.5A, FA). The MD maps, on the other hand, show excellent contrast in between GM and WM.

\subsection{Discussion}

In this work, we show how the $\mathrm{k}_{\mathrm{T}}$-dSSFP sequence achieves homogeneous diffusion-weighted SSFP whole-brain images at $9.4 \mathrm{~T}$ by improving $\mathrm{B}_{1}{ }^{+}$ homogenisation with the composite $\mathrm{k}_{\mathrm{T}}$-points pulses (Figure 4.2) method. The benefit of using these pulses, in comparison to a global single pulse, is an enhancement in the total signal intensity and improved homogeneity as shown in Figure 4.3A. The dark regions resulting from the destructive interference of the $\mathrm{RF}$ pulses are recovered (Figure 4.3B). It is important to mention that the improvement in $\mathrm{B}_{1}{ }^{+}$homogeneity and overall SNR was not only due to the better excitation profile but also due to the use of an efficient transmit and receiver coil (Figure 4.1), compared to earlier ex vivo human brain studies that used more standard head coils.

The improved $\mathrm{B}_{1}{ }^{+}$homogeneity, and hence signal, over a human brain hemisphere allowed us to exploit the advantages of $\mathrm{k}_{\mathrm{T}}-\mathrm{SSFP}$ diffusionweighting at UHF through the acquisition of high b-values at ultra-high resolution. As shown in Figure 4.4, effective b-values of $4000 \mathrm{~s} / \mathrm{mm}^{2}$ were acquired at 500 and $450 \mu \mathrm{m}$ isotropic resolution. An SNR decline of a factor of approximately 1.34 would be expected when the resolution is increased from 500 to $450 \mu \mathrm{m}$ isotropic. However, the hardware and sequence specifications (i.e. $\mathrm{G}_{\max }$ amplitude and EPI-factor) used for each case were different. As shown in Table 4.1, the $450 \mu \mathrm{m}$ resolution data was acquired with higher load on hardware components (i.e. highest gradient amplitude possible and higher duty cycle, therefore shorter TR/TE) with almost twice the heat-load in comparison to the $500 \mu \mathrm{m}$ data. At the same time, the heat-load needed to acquire this data was, at least, one quarter less than used in the $7 \mathrm{~T}$. The most feasible explanations are the magnetic field increment (from $7 \mathrm{~T}$ to $9.4 \mathrm{~T}$, therefore more signal) and the better signal efficiency from the custom RF-coil transmission and, especially, reception 
4 Human whole-brain diffusion MRI at 450 um post mortem with $k_{T^{-}} d S S F P$ and a specialised $9.4 T R F$-coil

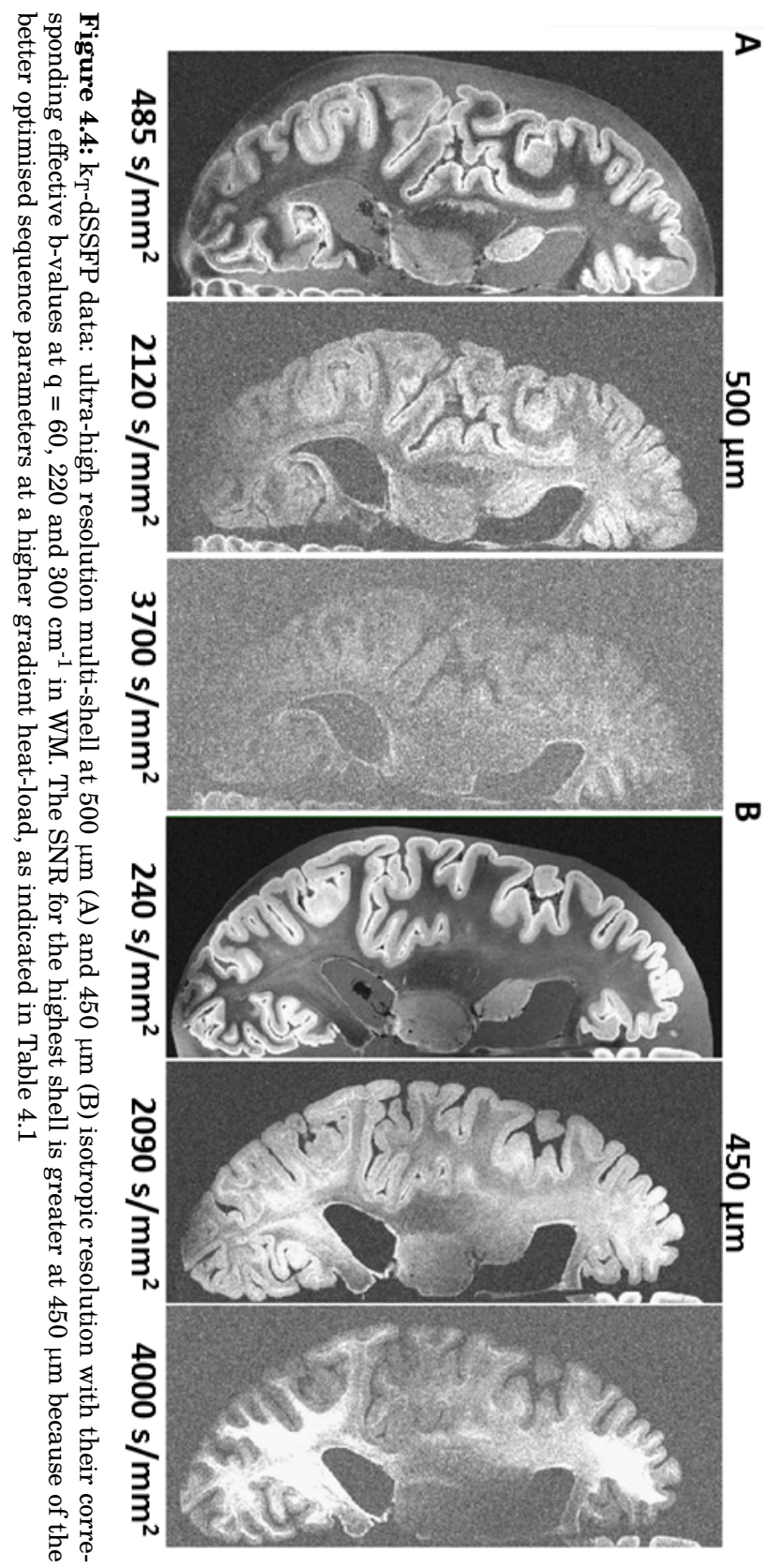



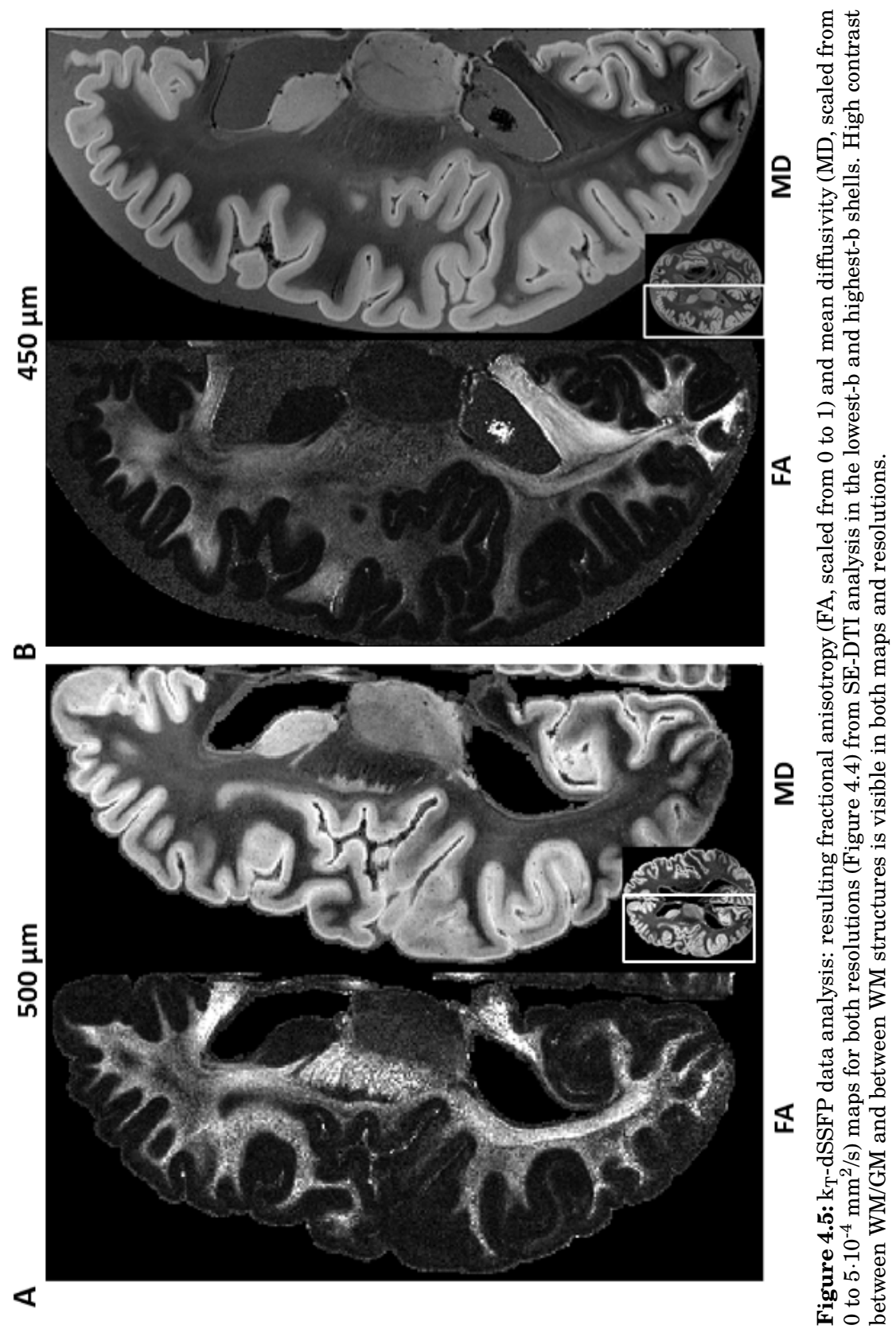
(Figure 4.1). These ultra-high resolution acquisitions $(450-500 \mu \mathrm{m})$ have enough SNR to be submitted to diffusion analysis and yield feasible results [115]. Preliminary SE-DTI analysis was performed in these data showing high contrast between GM/WM and in between WM structures (especially in the FA maps) (Figure 4.5). This shows that the $\mathrm{k}_{\mathrm{T}}$-dSSFP sequence can generate high-quality data in this SNR-challenged regime (moderate b-value at ultra-high resolution). However, multi-shell analysis of $\mathrm{k}_{\mathrm{T}}$-SSFP was not performed in this work. Nevertheless, it is feasible to acquire several q-values ranging from 60 (used as $q_{0}$ or $b_{0}$ ) to $300 \mathrm{~cm}^{-1}$ for multiple single-shell diffusion analyses. It is also important to note that due to the short acquisition time per volume (from $\approx 20$ min at 500 $\mu \mathrm{m}$ to $\approx 40 \mathrm{~min}$ at $450 \mu \mathrm{m}$ isotropic, Table 4.1 ), it is possible to acquire a considerable amount of diffusion directions, which can be beneficial for higher complexity modelling such as Ball\&Stick with two or three sticks or constrained spherical deconvolution [206]. Even though the amount and quality of the $\mathrm{k}_{\mathrm{T}}$-dSSFP data can be superior in comparison to PGSE, the highly complex signal modelling and dependency in parameters as $\mathrm{B}_{1}{ }^{+}$(i.e. flip angle), $\mathrm{T}_{1}$ and $\mathrm{T}_{2}$ can heavily bias the quantitative diffusion parameters, such as the ones obtained in Figure 4.5. Therefore, it is important to obtain an accurate $\mathrm{B}_{1}{ }^{+}$map when using the $\mathrm{k}_{\mathrm{T}}$-points pulses, as well as accurately estimated $\mathrm{T}_{1}$ and $\mathrm{T}_{2}$ maps.

\subsubsection{Outlook}

$\mathrm{k}_{\mathrm{T}}$-dSSFP data was acquired at 450-500 $\mu \mathrm{m}$ resolution with moderate $\mathrm{q}$ value of $300 \mathrm{~cm}^{-1}$ (or an effective $4000 \mathrm{~s} / \mathrm{mm}^{2}$ b-value). A combined multishell analysis in this dataset would perhaps allow improved tractography and crossing fibre modelling (c.f. Chapter 3). However, this would require a better estimation of $\mathrm{B}_{1}{ }^{+}, \mathrm{T}_{1}$ and $\mathrm{T}_{2}$ due to the highly complex signal behaviour of SSFP. Furthermore, given the low tolerable heat-load factor for the $500 \mu \mathrm{m}$ isotropic acquisition, it is feasible to optimise the sequence parameters further to improve even higher SNR. With further developments in data analysis and modelling this data can play an important role in mesoscale human connectomics and microstructure studies and help to bridge the gap between in vivo MRI studies and post mortem histology. 


\section{Comprehensive analysis and characterisation of the steady-state signal in the STEAM sequence ${ }^{1}$}

\footnotetext{
${ }^{1}$ In preparation for submission.
} 


\subsection{Introduction}

The Stimulated Echo Acquisition Method (STEAM) is an MR sequence introduced by Frahm and colleagues in 1985 [85]. It consists of three RF pulses with equal phase: the excitation, storing and recalling pulses. The combination of those pulses generates a controlled primary spin-echo (SE), stimulated echo (STE) and three extra echoes signals (called 2nd, 3rd and 4th SE). In comparison to the pulse-gradient spin-echo (PGSE) sequence, where the refocusing pulse (i.e. with $\pi$ rad flip angle) refocuses all the excited spins to generate the SE signal; in STEAM, the storing pulse refocuses a fraction of the total magnetisation to create the SE signal while moving the remaining fraction to the longitudinal plane. After a specific period called mixing time (TM), the recalling pulse places back the magnetisation sent to the longitudinal plane by the storing RF pulse into the transverse plane, generating the STE signal. The extra echoes are generated by refocusing the free induction decay (FID) signals from the storing and recalling pulses. The fraction of signal divided by the storing and the fraction stored recovered by the recalling pulses depend on their respective flip angles. Using $\pi / 2$ rad flip angles in both pulses, the storing RF pulse divides the total magnetisation by half, and this fraction is completely recovered by the recalling $\mathrm{RF}$ pulse afterwards.

The (hereafter only) primary SE and STE signals are analytically defined by solving the Bloch equation for STEAM, whose solutions are shown in Equation 5.1 and 5.2, respectively. These signal equations present an equal $\mathrm{T}_{2}$-weighting (dependent on the echo time, $\mathrm{TE}$ ) and total magnetisation component $\left(\mathrm{M}_{\mathrm{z}}\right.$, often approximated to 1$)$. Besides, an extra $\mathrm{T}_{1}$ weighting (dependent on TM) is only present in the STE signal. The resulting multi-weighting (or contrast) in the STE signal, and the equal $\mathrm{T}_{2}$ information that can be obtained from SE and STE signals, makes acquiring the SE signal seemingly redundant. Therefore, it is conventional to use the STEAM sequence with the SE signal spoiled (e.g. in reference [40]) for a reduced acquisition time (or TM) or, if the signal is acquired nevertheless, it is used as a normalisation component for the STE signal (e.g. see reference [138]). However, performing an independent analysis on the SE signal acquired in STEAM could be beneficial for microstructure analysis using diffusion MRI (dMRI). As illustrated in Figure 5.1, while the STE signal is diffusion-weighted by a diffusion time defined as $\Delta+\mathrm{TM}$, the SE signal is diffusion-weighted by $\Delta$. This difference in diffusion-weighting (low in SE and high in STE) could help in revealing microstructural information (see 
references $[45,153,52])$ if a joint analysis SE-STE is performed. Despite that, this type of analysis requires an explicit definition of $\mathrm{M}_{z}$ that not necessarily is simplified to the unit in dMRI, especially when repetition time (TR) and TM are lower than $\mathrm{T}_{1}$ (similar to the partial saturation effect for gradient echo sequence [99]).

In recent works, Alexander et al. 2013 [7] introduced an explicit definition for $M_{z}$ in the STE signal equation, and later it was modified in the work of Zhang et al. 2019 [238]. This factor is referred to as the steady-state component, which represents the fraction of the total magnetisation available when it is not fully relaxed (e.g. see reference [99]). This component shows to be $\mathrm{T}_{1}$-dependent and weighted by the time parameter called effective repetition time (eff-TR), defined in the work of Alexander et al. as the difference between the repetition time (TR) and TM: (TR - TM), and later in the work of Zhang et al. with an additional TE/2: (TR - TM - TE/2). The addition of the steady-state component in the STE signal (and therefore in the SE signal) increases the complexity of estimating the different signal components (or weightings, i.e. $\mathrm{T}_{1}$ ). And, it could imply acquiring data in a more densely sampled scheme of TR, TM, and TE. In addition to this increasing complexity in acquisition and analysis, it is not completely understood (or explicit) if this steady-state component is sensitive to other sequence parameters. For example, to flip angles (in comparison to the Equations 5.1 and 5.2, which affect the transverse component), to time regimes where TM is very close to TR or TE/2, or if the acquisition of the SE signal could influence it. At this point, it is not the acquisition per se affecting this component but it is the remnant SE signal before applying the recalling $\mathrm{RF}$ pulse. If this situation occurs, then this remnant transverse SE signal is sent to the longitudinal plane until TR is reached. Thus, understanding this signal component will help to predict accurately how the STE signal will behave in time regimes (i.e. TR) lower than $\mathrm{T}_{1}$. Moreover, in special cases where this signal is acquired in an MR system with a severe inhomogeneous $\mathrm{B}_{1}{ }^{+}, \mathrm{T}_{1}$ relaxometry performed at TM values sampled close to TE/ 2 and TR, and if the SE signal is used for joint microstructural dMRI analysis.

Therefore, this work aims to study the steady-state signal component in the STEAM sequence in further detail, with the purpose of describing how sensitive this component is in the aforementioned scenarios: in case of $\mathrm{B}_{1}{ }^{+}$inhomogeneity (i.e. angle dependence), time constraints and SE remnant contamination. Firstly, two detailed steady-state components are obtained by solving the Bloch equation for the longitudinal component $\left(\mathrm{M}_{\mathrm{z}}\right)$ in STEAM. Those equations represent the steady-state component when 
a remnant transverse SE signal affects this component (referred to as the unspoiled SE case) or not (referred to as the spoiled SE case). Those expressions are compared to define in which time and angle conditions the discrepancy between both models is maximum or minimum. Without losing generality, the full SE/STE signals equations with the unspoiled SE steady-state signal component added are then used. Second, these equations are analysed in function of normalised values, signal-to-noise ratio (SNR) and signal-to-noise ratio efficiency (SNR-eff) in an extended range of TM and TR (both normalised and not normalised by $\mathrm{T}_{1}$ ), and flip angles $\left(\alpha_{\mathrm{i}}\right)$ values. Finally, those signal models are compared with MR data acquired using a homogeneous material (i.e. phantom) and an ex vivo brain specimen at 9.4 T. This is because at ultra-high field (UHF) scanners, $\mathrm{T}_{1}$ increases and $\mathrm{B}_{1}{ }^{+}$inhomogeneity is more severe than in lower-field scanners. Therefore, conditions with variable flip angles and short timing parameters (i.e. TR and TM) in comparison to $\mathrm{T}_{1}$ can be better explored.

\subsection{Methods}

The STEAM sequence with diffusion gradients (diffusion-weighted STEAM, dSTEAM) is represented in Figure 5.1. Timing parameters such as the echo time (TE), repetition time (TR) and mixing time (TM) are represented as a function of $\tau_{1}, \tau_{2}$ and $\tau_{3}$ where $\mathrm{TE}=2 \tau_{1}, \mathrm{TM}=\tau_{2}$ and $\mathrm{TR}=\tau_{3}+\tau_{2}+$ $\tau_{1}$. The corresponding diffusion times (if needed) can be approximated as a function of $\tau_{1}$ and $\tau_{2}$, with the assumption that there are no time gaps between gradients. The transverse scalar component $\left(\mathrm{M}_{\mathrm{xy}}\right)$ of the magnetisation vector $\left(\mathbf{M}^{\mathrm{i}}\right)$ in the SE signal at echo time $\left(2 \tau_{1}\right)$ and in the STE signal at echo time $\left(2 \tau_{1}+\tau_{2}\right)$ is defined respectively as:

$$
\mathrm{SE}: \quad M_{x y}\left(\alpha_{i}, \tau_{1}, T_{2}\right)=M_{z} \sin \left(\alpha_{1}\right) \sin \left(\frac{\alpha_{2}}{2}\right)^{2} \exp \left(\frac{-2 \tau_{1}}{T_{2}}\right)
$$

$$
\text { STE: } \quad M_{x y}\left(\alpha_{i}, \tau_{1}, \tau_{2}, T_{1}, T_{2}\right)=0.5 M_{z} \prod_{i=1}^{3} \sin \left(\alpha_{i}\right) \exp \left(\frac{-2 \tau_{1}}{T_{2}}\right) \exp \left(\frac{-\tau_{2}}{T_{1}}\right)
$$

where the longitudinal scalar component of the total magnetisation vector, $\mathbf{M}^{\mathrm{i}}\left(\mathrm{M}_{\mathrm{z}}\right)$ is not explicitly defined. Therefore, we will derive this component here, in order to evaluate the steady-state behaviour of SE and STE signals over a range of parameters. For this derivation, it is necessary to solve the 


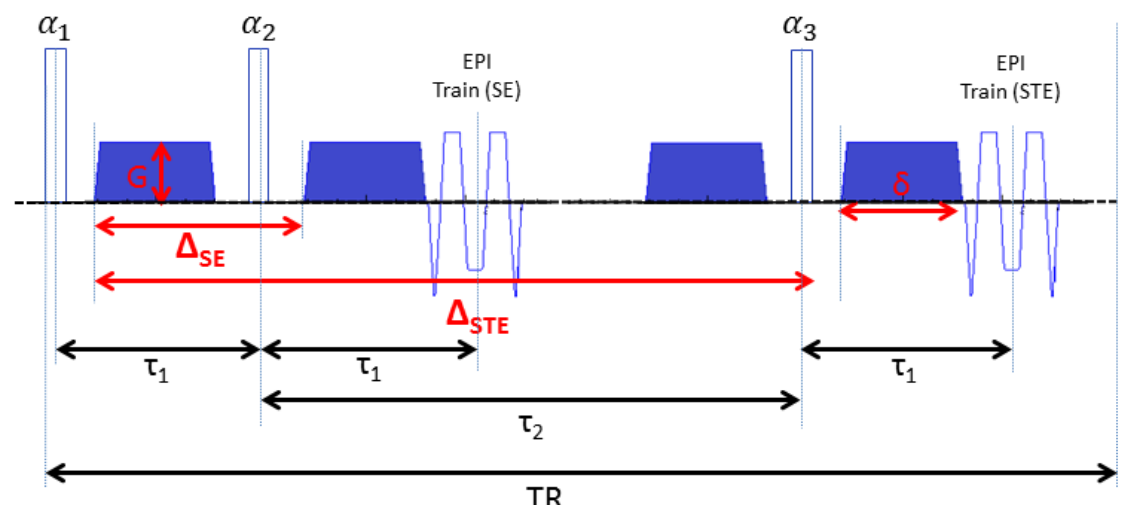

Figure 5.1: The diffusion-weighted STEAM sequence diagram for one repetition time (TR) with readout for the both (primary) SE signal at echo time $\left(2 \tau_{1}\right)$ and the STE signal at echo time plus mixing time $\left(2 \tau_{1}+\tau_{2}\right)$. RF pulses can be globally selective, generally with a segmented 3D EPI readout (shown here), or slice selective, generally with a single-shot 2D EPI readout. Corresponding diffusion parameters are also indicated: diffusion times for $\mathrm{SE}\left(\Delta_{\mathrm{SE}}\right)$ and STE $\left(\Delta_{\mathrm{STE}}\right)$, diffusion gradient amplitude $(\mathrm{G})$ and diffusion gradient duration $(\delta)$. For simplicity, it is assumed that the diffusion gradient duration is the same along all of them.

Bloch equation for $\tau=$ TR. Its solution then is defined by:

$$
M_{z}(\tau)=M_{z}^{0}\left(1-\exp \left(-\tau_{3} / T_{1}\right)\right)+M_{z}^{\alpha_{3}} \exp \left(-\tau_{3} / T_{1}\right)
$$

where $\tau_{3}=\mathrm{TR}-\left(\tau_{2}+\tau_{1}\right)=\mathrm{TR}-(\mathrm{TM}+\mathrm{TE} / 2)$, referred as the effective TR (as defined in reference [238]), is the remaining time after the application of the $\alpha_{3}$ RF-pulse, and $M_{z}^{\alpha_{i}}$ is hereafter equal to the resulting $\mathbf{M}_{\mathbf{z}}$ component of $\mathbf{M}^{\mathrm{i}}$ after the application of the $\alpha_{\mathrm{i}}$ RF-pulse. Obtaining the expanded form of Equation 5.3 (which will be used in this study) requires the addition of two assumptions. The first assumption is that the total signal in the STEAM sequence reached a steady-state condition (Equation 5.4-top). The second assumption is that there is no-remaining transverse magnetisation signal after each TR (Equation 5.4-bottom), i.e. there is complete spoiling just before each excitation pulse.

$$
\begin{aligned}
M_{z}\left(T R_{i}\right)=M_{z}\left(T R_{i-1}\right) & \text { and } \\
M_{x}\left(T R_{i}\right)=M_{x}\left(T R_{i-1}\right)=M_{y}\left(T R_{i}\right)=M_{y}\left(T R_{i-1}\right)=0 & \forall i>0
\end{aligned}
$$


Expanding Equation 5.3, and applying the assumptions from Equation 5.4, results in:

$$
\begin{array}{r}
M_{z}(T R)=M_{z}^{0}(T R) \\
-M_{z}\left(\sin \left(\alpha_{1}\right) \cos \left(\alpha_{2}\right) \sin \left(\alpha_{3}\right) \cos \left(\omega \tau_{1}\right) \cos \left(\omega \tau_{2}\right) E_{2}\left(\tau_{1}+\tau_{2}\right) E_{1}\left(\tau_{3}\right)\right. \\
+\sin \left(\alpha_{1}\right) \sin \left(\alpha_{2}\right) \cos \left(\alpha_{3}\right) \cos \left(\omega \tau_{1}\right) E_{2}\left(\tau_{1}\right) E_{1}\left(\tau_{2}+\tau_{3}\right) \\
-\sin \left(\alpha_{1}\right) \sin \left(\alpha_{3}\right) \sin \left(\omega \tau_{1}\right) \sin \left(\omega \tau_{2}\right) E_{2}\left(\tau_{1}+\tau_{2}\right) E_{1}\left(\tau_{3}\right) \\
+\cos \left(\alpha_{1}\right) \sin \left(\alpha_{2}\right) \sin \left(\alpha_{3}\right) \cos \left(\omega \tau_{2}\right) E_{2}\left(\tau_{2}\right) E_{1}\left(\tau_{1}+\tau_{3}\right) \\
\left.-\cos \left(\alpha_{1}\right) \cos \left(\alpha_{2}\right) \cos \left(\alpha_{3}\right) E_{1}\left(\tau_{1}+\tau_{2}+\tau_{3}\right)\right)
\end{array}
$$

Where $\omega$ is the corresponding (Larmor) frequency of $\mathbf{M}^{\mathrm{i}}, \mathrm{E}_{1}(\tau)=\exp \left(-\tau / \mathrm{T}_{1}\right)$, $\mathrm{E}_{2}(\tau)=\exp \left(-\tau / \mathrm{T}_{2}\right)$ for any time $\tau$, and $\mathrm{M}_{\mathrm{z}}{ }^{0}(\mathrm{TR})$ is the parameter containing all the components related to the full longitudinal magnetisation $\left(\mathrm{M}_{\mathrm{z}}{ }^{0}\right)$. Its corresponding expanded form is given by:

$$
\begin{array}{r}
M_{z}^{0}(T R)=M_{z}^{0}\left(\left(1-E_{1}\left(\tau_{3}\right)\right)+\left(1-E_{1}\left(\tau_{2}\right)\right) E_{1}\left(\tau_{3}\right) \cos \left(\alpha_{3}\right)\right. \\
+\left(1-E_{1}\left(\tau_{1}\right)\right) E_{1}\left(\tau_{2}+\tau_{3}\right) \cos \left(\alpha_{2}\right) \cos \left(\alpha_{3}\right) \\
\left.-\left(1-E_{1}\left(\tau_{1}\right)\right) E_{2}\left(\tau_{2}\right) E_{1}\left(\tau_{3}\right) \sin \left(\alpha_{2}\right) \sin \left(\alpha_{3}\right) \cos \left(\omega \tau_{2}\right)\right)
\end{array}
$$

where it is important to note that this parameter is not dependent on $\alpha_{1}$. Conventionally, it is expected that the three RF pulses are the same $\left(\alpha_{1}=\right.$ $\left.\alpha_{2}=\alpha_{3}=\alpha\right)^{2}$. This allows simplifying Equation 5.5 (and implicitly Equation 5.6) to:

$$
\begin{array}{r}
M_{z}(T R)=M_{z}^{0}(T R)-M_{z}\left(\sin (\alpha)^{2} \cos (\alpha) \cos \left(\omega \tau_{1}\right) \cos \left(\omega \tau_{2}\right) E_{2}\left(\tau_{1}+\tau_{2}\right) E_{1}\left(\tau_{3}\right)\right. \\
+\sin (\alpha)^{2} \cos (\alpha) \cos \left(\omega \tau_{1}\right) E_{2}\left(\tau_{1}\right) E_{1}\left(\tau_{2}+\tau_{3}\right) \\
-\sin (\alpha)^{2} \sin \left(\omega \tau_{1}\right) \sin \left(\omega \tau_{2}\right) E_{2}\left(\tau_{1}+\tau_{2}\right) E_{1}\left(\tau_{3}\right) \\
+\cos (\alpha) \sin (\alpha)^{2} \cos \left(\omega \tau_{2}\right) E_{2}\left(\tau_{2}\right) E_{1}\left(\tau_{1}+\tau_{3}\right) \\
\left.-\cos (\alpha)^{3} E_{1}\left(\tau_{1}+\tau_{2}+\tau_{3}\right)\right)
\end{array}
$$

Equation 5.7 shows that the resulting $\mathrm{M}_{\mathrm{z}}$ can be studied under two separated situations: (1) $M_{z}$ is dependent only on the timing and frequency by fixing the flip angles (i.e. $\alpha_{i}=\pi / 2$ ) or (2) $M_{z}$ is dependent only on the timing

\footnotetext{
${ }^{2}$ In experimental conditions, $\alpha \approx \alpha_{0} \pm \varepsilon_{\alpha}$ (with $\varepsilon_{\alpha}$ the error). This analysis is still valid if the trigonometric functions containing this variable are expanded using first order Taylor's expansion.
} 
and flip angles by assuming an isocromat condition (the proton density is uni-valued only at Larmor frequency $\left.\omega_{0}, \rho(\omega)=\delta\left(\omega-\omega_{0}\right)^{3}\right)$. Since timing and flip angles are parameters that can be manipulated by the user during a MR scanning session, the second situation will be explored in this work. Removing the $\omega$-dependency from Equation 5.7 requires an integration over frequency along all the possible values (Equation 5.8-top). Since we are considering the isocromat condition previously mentioned, some mathematical simplifications can be applied (Equation 5.8).

$$
\begin{array}{r}
\int_{0}^{\infty} M_{z}\left(\alpha, \tau_{1}, \tau_{2}, \omega\right) \delta(\omega) d \omega=M_{z}\left(\alpha, \tau_{1}, \tau_{2}\right) \\
\int_{0}^{\infty} \sin (\omega \tau)^{n} \delta(\omega) d \omega=0 ; \int_{0}^{\infty} \cos (\omega \tau)^{n} \delta(\omega) d \omega=1 \quad n>0 .
\end{array}
$$

Using these simplifications Equation 5.7 reduces to:

$$
\begin{array}{r}
M_{z}(T R)=M_{z}^{0}(T R) \\
-M_{z}\left(\sin (\alpha)^{2} \cos (\alpha) E_{2}\left(\tau_{1}+\tau_{2}\right) E_{1}\left(\tau_{3}\right)+\sin (\alpha)^{2} \cos (\alpha) E_{2}\left(\tau_{1}\right) E_{1}\left(\tau_{2}+\tau_{3}\right)\right. \\
\left.+\cos (\alpha) \sin (\alpha)^{2} E_{1}\left(\tau_{1}+\tau_{3}\right) E_{2}\left(\tau_{2}\right)-\cos (\alpha)^{3} E_{1}\left(\tau_{1}+\tau_{2}+\tau_{3}\right)\right)
\end{array}
$$

Taking the first assumption in Equation 5.4 into Equation 5.9, an expression for $M_{z}(0)$ or for the resulting steady-state longitudinal component is obtained:

$$
M_{z}\left(\alpha, \tau_{i}\right)=M_{z}^{0}\left(\frac{A\left(\alpha, \tau_{i}, \beta=1\right)}{B\left(\alpha, \tau_{i}, \beta=1\right)}\right)
$$

where:

$$
\begin{array}{r}
\boldsymbol{A}\left(\alpha, \tau_{i}, \beta\right)=\left(1-E_{1}\left(\tau_{3}\right)\right)+\left(1-E_{1}\left(\tau_{2}\right)\right) E_{1}\left(\tau_{3}\right) \cos (\alpha) \\
+\beta\left(1-E_{1}\left(\tau_{1}\right)\right)\left(E_{1}\left(\tau_{2}+\tau_{3}\right) \cos (\alpha)^{2}-E_{2}\left(\tau_{2}\right) E_{1}\left(\tau_{3}\right) \sin (\alpha)^{2}\right) \\
\boldsymbol{B}\left(\alpha, \tau_{i}, \beta\right)=1+\sin (\alpha)^{2} \cos (\alpha) E_{1}\left(\tau_{3}\right)\left(\beta E_{2}\left(\tau_{1}+\tau_{2}\right)\right. \\
\left.+E_{2}\left(\tau_{1}\right) E_{1}\left(\tau_{2}\right)+\beta E_{1}\left(\tau_{1}\right) E_{2}\left(\tau_{2}\right)\right)-\cos (\alpha)^{3} E_{1}\left(\tau_{1}+\tau_{2}+\tau_{3}\right)
\end{array}
$$

Equation 5.10 represents the total available signal in the STEAM sequence when the steady-state is achieved. The parameter $\beta$ introduced in this equation will be useful to differentiate the longitudinal component between

\footnotetext{
${ }^{3} \delta(\mathrm{x})$ is the Kronecker delta function, where $\delta(\mathrm{x})=1$ for $\mathrm{x}=0$ and 0 elsewhere.
} 
when a remaining SE signal contributes to the longitudinal component due to the recalling RF pulse or not. For the case in which the SE signal is affecting the longitudinal component (hereafter referred as unspoiled SE), the parameter $\beta$ will be evaluated to 1 . However, if the SE signal decays fully or it is crushed before the recalling RF pulse (hereafter referred as spoiled SE), all the components related to $\cos \left(\omega \tau_{2}\right)$ or $\sin \left(\omega \tau_{2}\right)$ in Equation 5.5 are simplified. The resulting longitudinal component is equal to 5.10 but with the parameter $\beta$ evaluated to 0 :

$$
M_{z}\left(\alpha, \tau_{i}\right)=M_{z}^{0}\left(\frac{A\left(\alpha, \tau_{i}, \beta=0\right)}{B\left(\alpha, \tau_{i}, \beta=0\right)}\right)
$$

In ideal conditions, where a perfect set of $\pi / 2$-rad pulses are used in STEAM, equations 5.10 and 5.12 are reduced (and become only timing-dependent) respectively to:

$$
\begin{array}{cl}
\text { Unspoiled SE: } & \left.M_{z}\left(\tau_{i}\right)=M_{z}^{0}\left(1-\left(1+\left(1-E_{1}\left(\tau_{1}\right)\right) E_{2}\left(\tau_{2}\right)\right)\right) E_{1}\left(\tau_{3}\right)\right) \\
& \text { Spoiled SE: } \quad M_{z}\left(\tau_{3}\right)=M_{z}^{0}\left(1-E_{1}\left(\tau_{3}\right)\right)
\end{array}
$$

Equation 5.14 recovers the equation defined firstly by Alexander et al. [7] and extended (by adding the TE/2 term) by Zhang et al. [238]. Achieving this equation here shows under which assumptions it was obtained: perfect $\pi / 2$-rad flip angles, spoiled SE and isocromat. Therefore, as the goal of this work, we will take a step further by studying the equations 5.10 in a broader range of parameter values, emphasising the effects of short TR's and varying flip angles, and comparing the signal behaviour with specific STEAM acquisitions using a cylindrical phantom and an ex vivo human brain specimen.

\subsubsection{Modelling and acquisition}

This work will use the full STE and SE signal equations (equations 5.1 and 5.2) with an explicit $\mathrm{M}_{\mathrm{z}}$ component added (equations 5.10 and 5.12) to model the signal behaviour in function of the timing parameters TR-TM-T ${ }_{1}$ and flip angles $\left(\alpha_{\mathrm{i}}\right)$. The signal modelling aims:

1. To study the difference in the total STE signal where we assume unspoiled and spoiled SE signal conditions. This will be performed for a range of TR's $(0.5,1,2$ and $4 \mathrm{~s})$ and TM values ( 0 to the correspond- 
ing upper limit defined by TR), and flip angle values ( $\pi / 3, \pi / 2$, and $5 \pi / 9 \mathrm{rad}$ ). The results will determine whether the following tests require to be performed separately for unspoiled and spoiled SE signal conditions.

2. To study the flip angle dependence of the STE signal by varying the excitation RF pulse flip angle with fixed storing/recalling flip angles $\left(\alpha_{1}-\pi / 2-\pi / 2\right)$ or varying the storing and recalling pulses with fixed excitation flip angle $\left(\pi / 2-\alpha_{2}-\alpha_{3}\right)$. This investigates whether the combination of $\pi / 2$ flip angles results in the highest STE echo signal achievable in STEAM for a short TR regime.

3. To estimate an optimum TR for a fixed TM in such a way that the highest SNR efficiency (SNR-eff $\sim \mathrm{SNR} / \sqrt{\mathrm{TR}}$ ) in the perfect flip angles scenario $\left(\alpha_{i}=\pi / 2\right.$, i.e. using equations 5.13 and 5.14) can be achieved.

4. To determine how the STE signal model differs in the TM-TR space (e.g. variable TM-TR combinations) when the signal is in a steadystate regime $\left(M_{z}\right.$ defined by Equation 5.10) or it is fully recovered $\left(M_{z}\right.$ $\sim 1$ ).

\subsubsection{Phantom and ex vivo acquisitions}

To validate the presented signal models, MRI data was acquired (SE and STE images) with the STEAM sequence using a homogeneous phantom (with a relatively long $\mathrm{T}_{1}$, over one second) and an ex vivo human brain sample (with a relatively short $\mathrm{T}_{1}$, lower than one second). Whereas the phantom was used to study the signal decay in a homogeneous $T_{1}$ specimen, the human brain specimen was used to study the SE-STE signals in a broader range of $T_{1}$ and $T_{2}$ values. The MRI phantom data were acquired using a cylindrical container $(8 \mathrm{~cm}$ radius $\mathrm{x} 8 \mathrm{~cm}$ height) filled with phosphate buffer saline (PBS) $+0.01 \%$ azide and the ex vivo whole human brain MRI data were acquired using a formalin-fixed whole-brain specimen from a subject without known neurological or psychiatric disease, which was previously used (see reference [88] or Chapter 3). For both studies, the images were acquired in a $9.4 \mathrm{~T} 82 \mathrm{~cm}$ bore Siemens MAGNETOM research scanner (Siemens Healthineers, Erlangen, Germany) with maximum gradient amplitude of $80 \mathrm{mT} / \mathrm{m}$ and maximum slew rate of $330 \mathrm{~T} / \mathrm{m} / \mathrm{s}$ 
per physical axis. For RF transmission and reception, a custom-built 16channel cylindrical coil [180] and 24-channel whole-brain coil [174] were used, for phantom and ex vivo brain acquisitions, respectively. Table 5.1 lists the sequence parameters used, relevant for the comparison with the theoretical signal modelling.

\begin{tabular}{|l|c|c|}
\hline Parameters/Study & Cylindrical Phantom & Specimen 1 \\
\hline$<\mathrm{T}_{1}>(\mathrm{s})$ & 4.000 & 0.450 \\
$<\mathrm{T}_{2}>(\mathrm{s})$ & 0.016 & 0.010 \\
$\mathrm{TM}\left(\tau_{2}\right)(\mathrm{s})$ & $0.03,0.04$, & $0.06,0.90,0.12$, \\
$\mathrm{TE}\left(2 \tau_{1}\right)(\mathrm{s})$ & $0.10: 0.10: 0.30$ & $0.18,0.26,(0.34,0.42)$ \\
$\mathrm{TR}\left(\tau_{1}+\tau_{2}+\tau_{3}\right)(\mathrm{s})$ & 0.023 & 0.014 \\
\hline
\end{tabular}

Table 5.1: Parameter values used for steady-state signal behaviour in phantom and ex vivo brain studies. Averaged $\mathrm{T}_{1}$ and $\mathrm{T}_{2}$ for the ex vivo brain are based on estimations in WM and for the phantom based on the embedding solution of the ex vivo specimen (Chapter 3)

It can be argued that the SE signal is a more specific measure of the steadystate component in STEAM than the STE signal, for the following two reasons:

- As reported, theoretically (e.g. see references [85, 40, 133] and others, and also shown in Equation 5.1) the transverse SE signal component is only dependent on $\mathrm{TE}$ (which is kept constant during the entire study) and expected to be invariant under TM. However, if the SE signal presents a variation on TM, then it is explained by the change in the $\mathrm{M}_{\mathrm{z}}$ component.

- In contradistinction, the STE signal is highly dependent on TM, as shown in Equation 5.2. The TM not only weights the STE signal by $\mathrm{T}_{1}$ during storing but also weights the signal due to diffusion (i.e. in the diffusion time, Figure 5.1). Therefore, since the $\mathrm{M}_{\mathrm{z}}$ is also dependent on TM, the STE signal is affected by TM simultaneously in three different ways and disentangling those components increases in difficulty exponentially.

As a consequence, isolating steady-state component contributions is far easier in the SE than the STE signal. Therefore, we will report both STE 
and SE signals and focus the interpretation of steady-state effects on the SE signal results.

\subsection{Results}

This section is divided into two subsections. The first subsection will present the modelled signal under the influence of the timing parameters TR-TM$\mathrm{T}_{1}$ and flip angles, with fixed TE- $\mathrm{T}_{2}$ and the remaining parameters normalised (e.g. proton density and transmit/receiver profiles). The second subsection will present the comparison between the modelled signal and experimental MR STEAM (i.e. SE signal) data acquired in phantom and ex vivo human brain samples.

\subsubsection{Steady-state signal dependencies and total signal behaviour}

Figure 5.2 shows the normalised difference between the STE signals modelled with the steady-state component in the cases of unspoiled (Equation 5.10) and spoiled (Equation 5.12) SE signals, for four different $T_{1}$ 's (from 0.25 to $2.0 \mathrm{~s}$ ). These $\mathrm{T}_{1}$ 's were chosen from a range of values typical for the in vivo (e.g. see reference [223]) and ex vivo (e.g. see reference $[170,88]$ ) human brain. For consistency, those values are used throughout this work. Fixed TR's of 0.5, 1, 2 and $4 \mathrm{~s}$ (in columns) are used to obtain a ratio of TR/T $\mathrm{T}_{1}$ from 0.25 (TR: $0.5 \mathrm{~s}-\mathrm{T}_{1}: 2.0 \mathrm{~s}$ ) to 16 (TR: $4.0 \mathrm{~s}-\mathrm{T}_{1}: 0.25 \mathrm{~s}$ ). Three different flip angles (equal for all the pulses in STEAM) are used (in rows): $\pi / 3 \mathrm{rad}(60$ degrees), $\pi / 2 \mathrm{rad}$ (90 degrees) and $5 \pi / 9 \mathrm{rad}$ (100 degrees), which represent the range of flip angles that might be expected in an MR scanner due to $\mathrm{B}_{1}{ }^{+}$inhomogeneity (with a reference flip angle of $\pi / 2 \mathrm{rad}$, e.g. in reference [208]). For all the TR/flip angles combinations, the difference between STE signals is the highest in the short TM regime. Moreover, the greatest differences are observable for long $\mathrm{T}_{1}$ 's where both signal estimations differ from almost $-15 \%$ at low flip angles ( $\pi / 3 \mathrm{rad}$, short TR) to $+15 \%$ at high flip angles ( $5 \pi / 9 \mathrm{rad}$, short TR). In the case of flip angles at $\pi / 2 \mathrm{rad}$, the difference between models almost vanishes (less than $1 \%$ ) for any TR. In a general perspective, the difference between models decreases with TM until the point where both models become equal. This can be explained by the $\mathrm{E}_{2}(\mathrm{TM})$ factor in Equation 5.10 (or more visible in Equation 5.13) which approximates to near zero when $T M>T_{2}$. Since the signal models are equal for most of the TM-TR ranges, we will focus on showing results for the unspoiled SE case (equations 5.10 and 5.13) which repre- 
sents, nevertheless, a generalised version of the steady-state component in STEAM. But it is important to take precautions in the signal study for the very short TM and TR regimes.

Figure 5.3 shows the resulting SNR efficiency (SNR-eff) of the STE signal for two $\mathrm{TM} / \mathrm{T}_{1}$ 's ratios: 0.25 and 2.0 , as a function of the $\mathrm{TR} / \mathrm{T}_{1}$ ratio and flip angles. Figure 5.3A shows four SNR-eff curves at different excitation flip angles ( $\pi / 3, \pi / 2,2 \pi / 3$ and $5 \pi / 6$ rads) with fixed storing and recalling flip angles (equal to $\pi / 2 \mathrm{rad}$ ). For both $\mathrm{TM} / \mathrm{T}_{1}$ ratios, the excitation flip angle acts as a scaling factor which results in scaled SNR-eff profiles; moreover when symmetric flip angles with respect to $\pi / 2 \mathrm{rad}$ are used (i.e. $\pi / 3$ and $2 \pi / 3 \mathrm{rad}$ in this case), the SNR-eff profiles are equal. This behaviour is expected because this RF pulse just excites the desired amount of spins from the longitudinal plane, achieving its maximum at $\pi / 2$ rad flip angle. Figure 5.3B shows, on the other hand, the variation of the SNR-eff in the function of the storing and recalling flip angles (also at $\pi / 3, \pi / 2,2 \pi / 3$ and $5 \pi / 6$ rads) with fixed excitation flip angle (equal to $\pi / 2$ ). For both $\mathrm{TM} / \mathrm{T}_{1}$ ratios, in comparison with 5.3A, the effect of both RF pulses scales and modifies the signal behaviour. In this particular scenario, the maximum SNR-eff efficiency is not necessarily achieved when both flip angles are $\pi / 2$ $\mathrm{rad}$. In fact, for the long $\mathrm{TM} / \mathrm{T}_{1}$ and short $\mathrm{TR} / \mathrm{T}_{1}$ case (when $\mathrm{TR} / \mathrm{T}_{1}-\mathrm{TM} / \mathrm{T}_{1}$ $<1$ ), using lower flip angles results in a higher SNR-eff. 


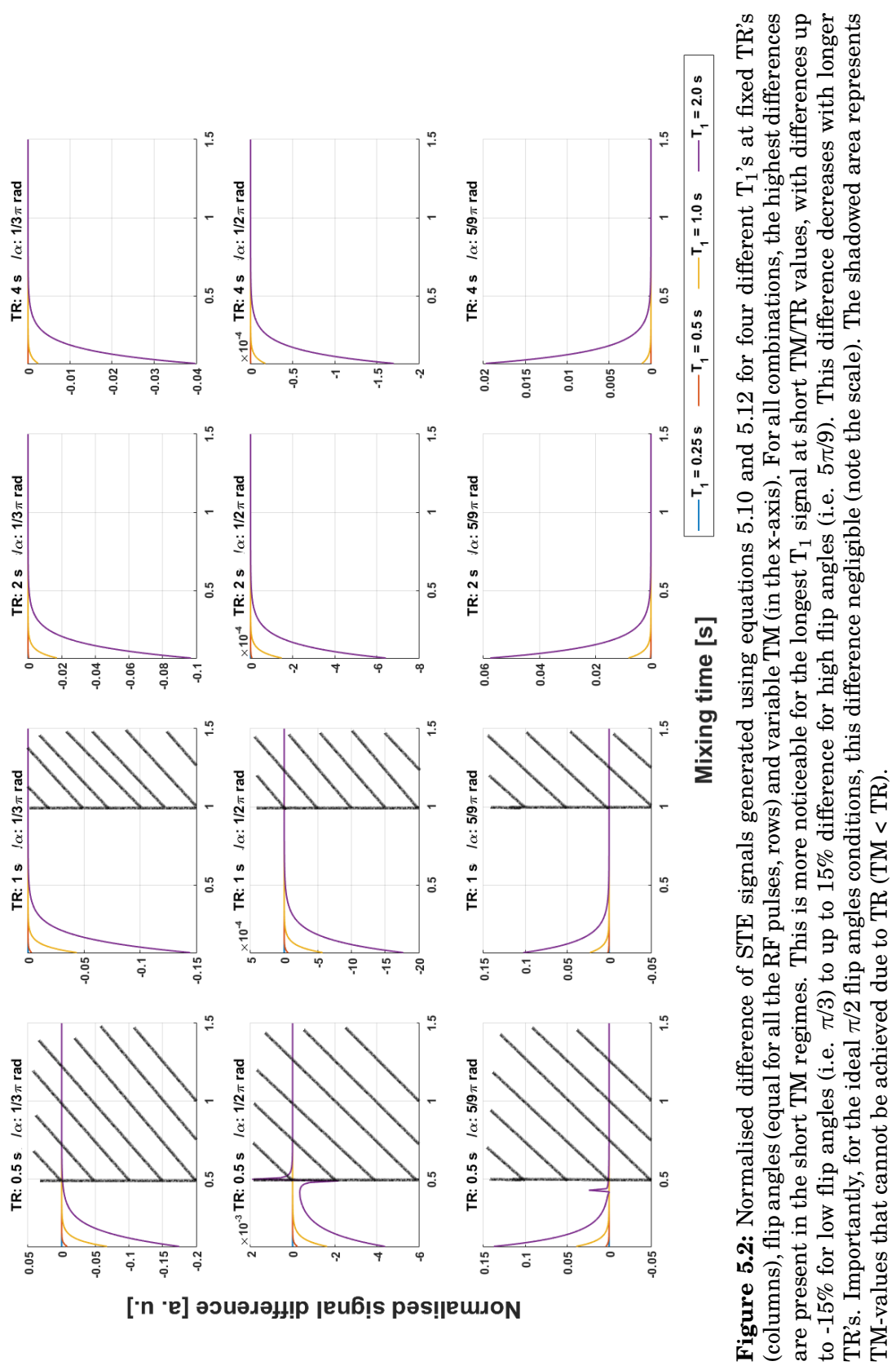


A

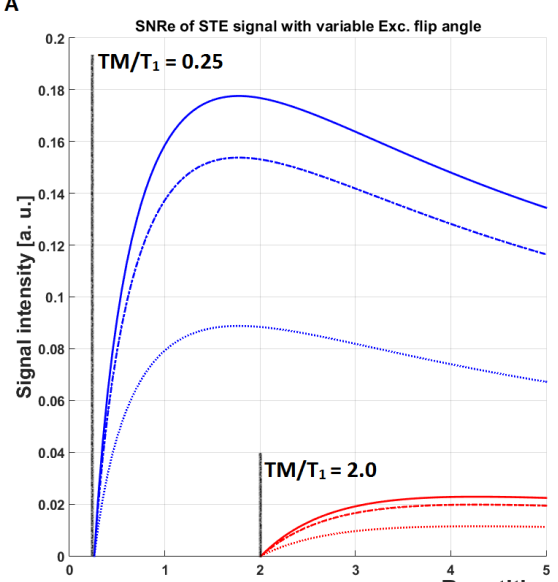

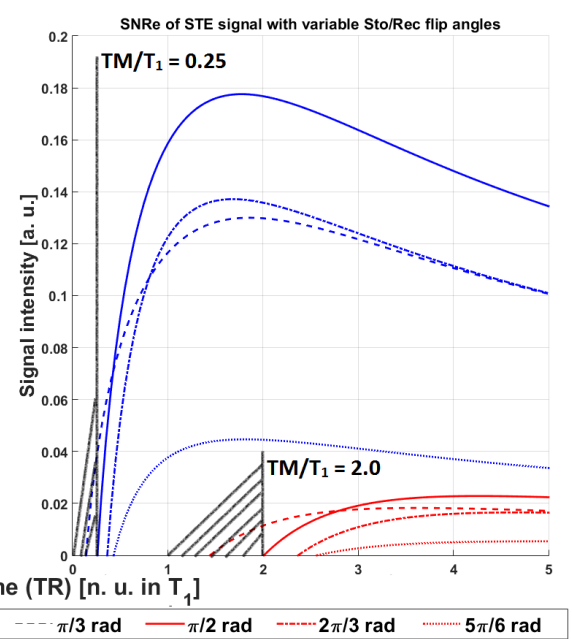

Figure 5.3: SNR-eff behaviour as a function of $T R / T_{1}$ with fixed $T M / T_{1}$ ratios $(0.25$ - blue curve, and 2.0 - red curve): (A) for variable excitation and fixed storing and recalling flip angles (equal to $\pi / 2$ ). For both ratios, the excitation flip angle just scales the total SNR-eff and achieves its maximum at $\pi / 2$. (B) For variable storing and recalling (both equal), and fixed excitation flip angles (equal to $\pi / 2$ ). In this case flip angle variation not only scales but also modifies the SNR-eff behaviour. The maximum SNR-eff is still achieved for flip angles equal to $\pi / 2$. However, this is not true in the range of (TR - TM)/ $\mathrm{T}_{1}<1\left(\cdot \mathrm{T}_{1}\right)$, where SNR-eff is higher when smaller flip angles are used.

Figure 5.4 shows another perspective of the SNR-eff behaviour, but only as a function of timing parameters (TM/ $\mathrm{T}_{1}$ and TR/ $\mathrm{T}_{1}$ ratios) with equally fixed flip angles $(\pi / 2 \mathrm{rad})$. Figure $5.4 \mathrm{~A}$ shows the SNR-eff profile in the $\mathrm{TM} / \mathrm{T}_{1}-\mathrm{TR} / \mathrm{T}_{1}$ space, where the highest $\mathrm{SNR}$-eff is achieved in the region close to a $\mathrm{TR} / \mathrm{T}_{1}$ ratio of 1.4 and $\mathrm{TM} / \mathrm{T}_{1}$ ratio lower than 0.25 . The SNReff decays slower along $\mathrm{TR} / \mathrm{T}_{1}$ than along $\mathrm{TM} / \mathrm{T}_{1}$ as shown in Figure $5.4 \mathrm{~B}$, resulting in a relatively broad maximum optimal SNR-eff peaks. By selecting the maximum SNR-eff values per $\mathrm{TM} / \mathrm{T}_{1}-\mathrm{TR} / \mathrm{T}_{1}$ (Figure $5.4 \mathrm{~A}$, white dashed line), it is possible to obtain a linear relationship between $\mathrm{TM} / \mathrm{T}_{1^{-}}$ $\mathrm{TR} / \mathrm{T}_{1}$ defined in Equation 5.15. This relationship is equally applicable for the unspoiled and spoiled SE signal model.

Non-spoiled \& spoiled: $\quad \mathrm{TR}=1.424 \mathrm{TM}+1.463 \quad\left(R^{2}: 0.9971\right)$ 

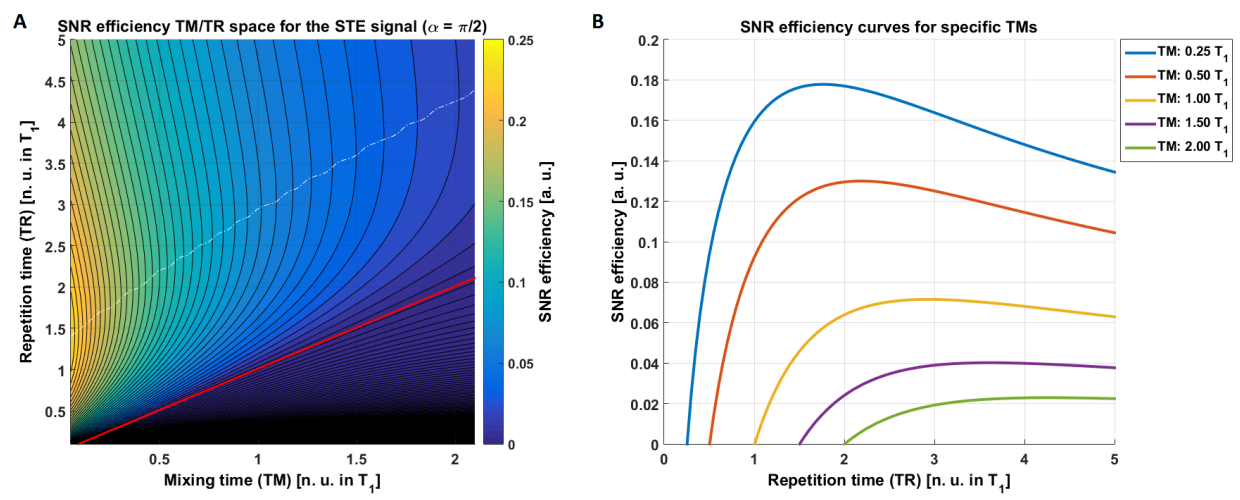

Figure 5.4: SNR-eff behaviour as a function of $T R / \mathrm{T}_{1}-\mathrm{TM} / \mathrm{T}_{1}$ ratios with fixed flip angles (all equal to $\pi / 2$ ): In (A), the SNR-eff is represented in the TM/TR space where the maximum is achieved for $\mathrm{TR} / \mathrm{T}_{1}$ ratio around 1.4 and $\mathrm{TM} / \mathrm{T}_{1}$ lower than 0.25 . The red line indicates the sequence limitations in the combination TR/TM (TM < TR - TE) with the right side forbidden. The white dashed line indicates the maximum value for each isocurve, which can be modelled with Equation 5.15. In (B), it shows some discrete curves from (A) for different $\mathrm{TM} / \mathrm{T}_{1}$ ratios. For any $\mathrm{TM} / \mathrm{T}_{1}$ ratio, the SNR-eff reaches a maximum and afterwards decays steadily. The maximum for each point can be obtained by using Equation 5.15.

Figure 5.5 shows, on the other hand, the contribution of each component of the modelled signal equation: the longitudinal (first row) and the transverse (second row) STE signal components, including how it is compared with the full STE signal model (third row, as shown in the previous results). All of them are shown in the TM/TR space at $\pi / 2 \mathrm{rad}$ flip angles. The longitudinal component behaves approximately linear with (TR-TM) for all the $\mathrm{T}_{1}$ 's (in columns). As expected, the value of this component is higher for long effective-TR values ( $\sim \mathrm{TR}-\mathrm{TM}$ ) and short $\mathrm{T}_{1}$ 's (to the limit where $\mathrm{M}_{\mathrm{z}}=1$ ). In contrast, the transverse signal decays exponentially and it is completely insensitive to TR. When both components are multiplied, the resulting STE signal behaves exponentially along TR and TM. It should be noted that the total STE signal is then modulated by two different mechanisms, which are highly dependent on $\mathrm{T}_{1}$. While the longitudinal component recovers significantly faster for short $T_{1}$, at the same time it decays quickly with increasing TM. Therefore, the total signal available in STE for very short to short $\mathrm{T}_{1}$ 's is strongly attenuated and mostly (if not only) dependent on TM. However, for the medium and longer $\mathrm{T}_{1}$ 's values, there is a broader combination of TM-TR times where the signal is highly dependent on TM and TR. Such dependency can even allow obtain- 
ing equal signal intensities at different TM-TR combinations.

The dependency shown by TM-TR-T $\mathrm{T}_{1}$ in the previous figure (Figure 5.5) is further illustrated in Figure 5.6. This figure shows how the STE signal decays for five different $\mathrm{T}_{1}$ 's at fixed TR's (in columns). By decreasing TR, a higher attenuating effect for longer $\mathrm{T}_{1}$ 's (e.g. $\mathrm{T}_{1}=2 \mathrm{~s}$ and $4 \mathrm{~s}$ in purple and green curves, respectively) in comparison to shorter $\mathrm{T}_{1}$ 's (e.g. $\mathrm{T}_{1}=1$ $\mathrm{s}$ in yellow) is observed. This attenuation in long $\mathrm{T}_{1}$ 's can reach intensity values (especially at $\mathrm{TM}=0 \mathrm{~s}$ ) below the point of shorter- $\mathrm{T}_{1}$ 's (e.g. the purple decay curve falls progressively lower below the yellow curve, and even slower for the green curve decay). This effect is explained by showing that the total magnetisation is not fully recovered for long $\mathrm{T}_{1}$ 's and decaying slower in comparison with short $\mathrm{T}_{1}$ 's where the total magnetisation is already (if not fully) recovered but decaying faster. Given the fact that those high $\mathrm{T}_{1}$ 's are commonly present in water, cerebrospinal fluid and blood, this interaction results in a significant attenuation of these substances in the short TR STEAM signal regime. This also means that the STE (and $\mathrm{SE}$ ) signal for different $\mathrm{T}_{1}$ 's can match under certain $\mathrm{TM} / \mathrm{TR}$ combinations. For example, there exists a specific TM value, at short TR, where the signal of the longest $T_{1}$ 's (purple and green curves) is equal for some of the shortest $\mathrm{T}_{1}$ 's curves.

\subsubsection{Experimental STEAM data analysis}

Figure 5.7 shows the STEAM SE images obtained from a homogeneous phantom acquired in a $9.4 \mathrm{~T}$ scanner at two different TR's $(0.35$ and 0.70 s) and several TM's (0.030 to $0.300 \mathrm{~s})$. This specimen was used to characterise the variation of the SE signal, for any arbitrary TM-TR values, as a result of the flip angle fluctuation (i.e. $\mathrm{B}_{1}{ }^{+}$-dependency, as shown in the inset). Figure 5.7A shows qualitatively how the signal decays in the images with respect to TM but increases at increasing TR. This is not properly shown since the images at $\mathrm{TR}=0.70 \mathrm{~s}$ were scaled twice the value in comparison to the signal scale at $\mathrm{TR}=0.35 \mathrm{~s}$. This was done with the purpose to determine, if possible, other qualitative differences between images at different TR's beyond signal scaling. As observed in Figure 5.7B, in where signal profiles of the images shown in 5.7A were plotted, no variation was observed between images. It corroborates the established qualitative observations, in which it is indeed an increment in signal with increasing TR in almost 1.5 times (e.g. in voxel position 16 , from 1.2 to $1.8 \cdot 10^{5}$ units) 


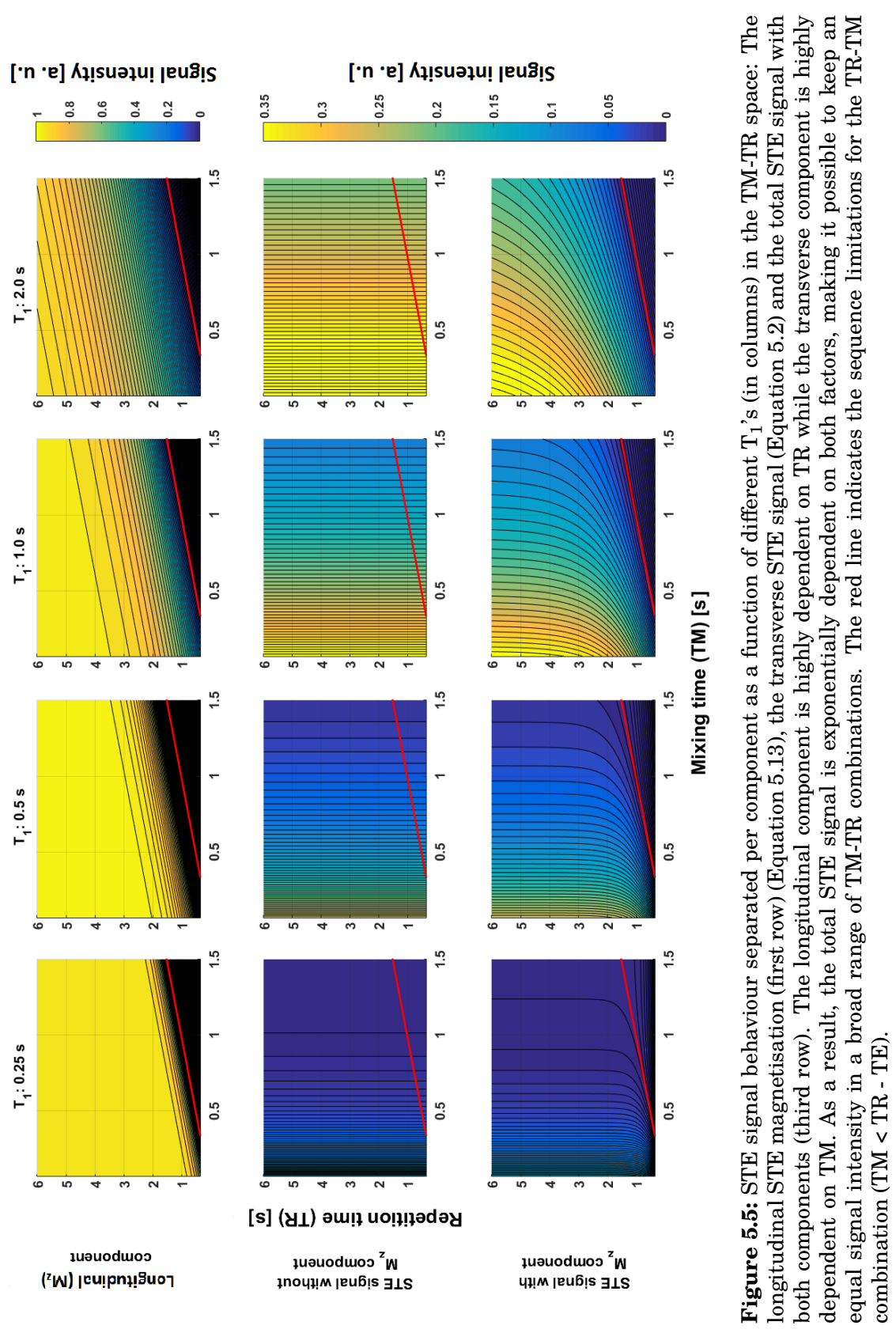


5 Comprehensive analysis and characterisation of the steady-state signal in the STEAM sequence

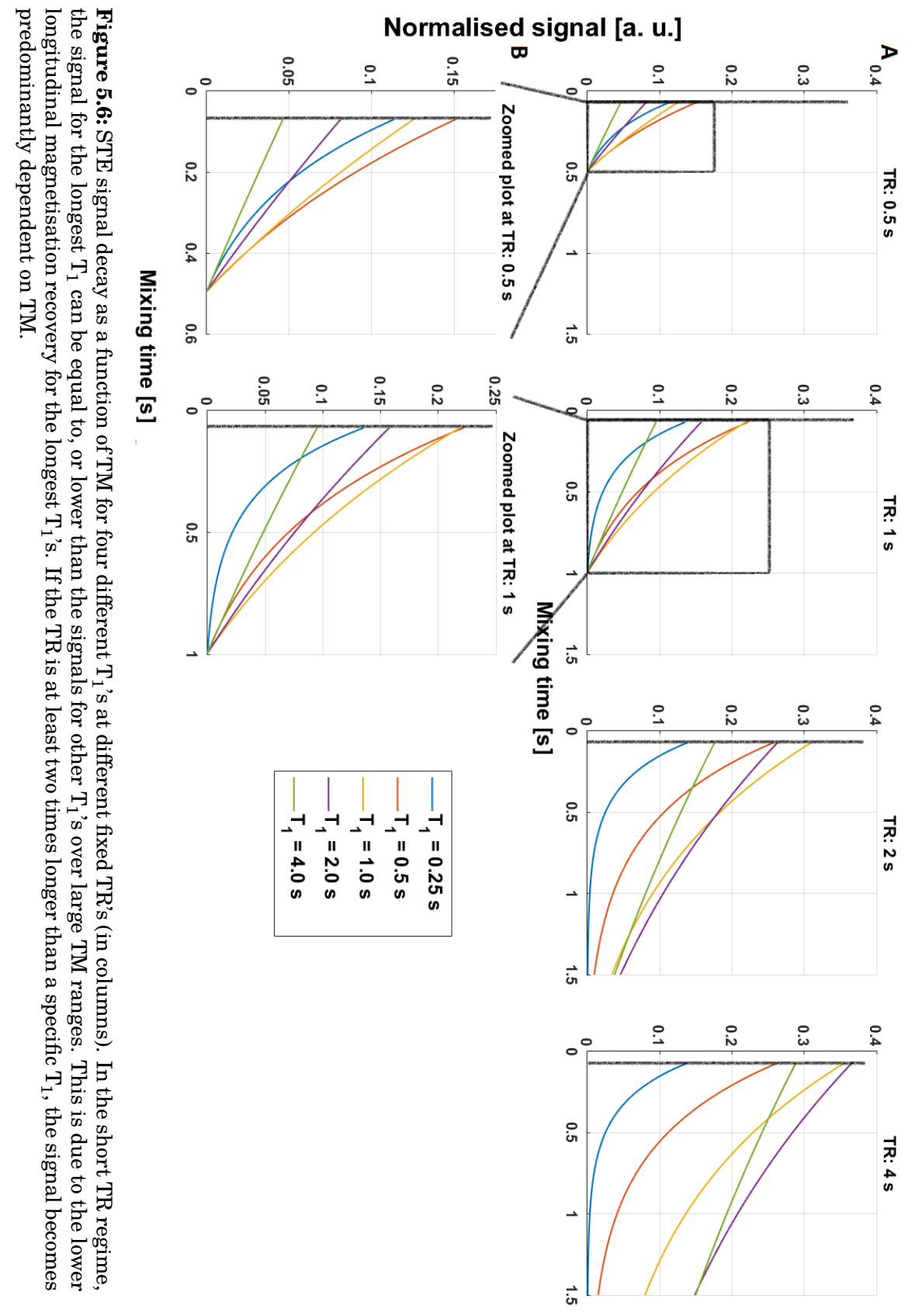


with a subtle difference in signal profiles at each TM. At the same time, the increment in TR just scales the SE signal proportionally, as demonstrated in Figure 5.5, top row. As argued above, the SE signal decay in function of TM can only be explained by the longitudinal component variation. Moreover, the SE signal is almost identical for the first three TM's $(0.03,0.042$ and $0.1 \mathrm{~s}$ ) which can be explained by the fact that the $\mathrm{T}_{1}$ value for the specimen is long enough to ensure a slow longitudinal decay. That is, for a $\mathrm{T}_{1}$ (far) above $0.7 \mathrm{~s}$, the $\left(1-\mathrm{E}_{1}\left(\tau_{3}\right)\right)$ component from Equation 5.14 is equal to $0.35,0.34$ and 0.28 at $\mathrm{TR}=0.35 \mathrm{~s}$, and equal to $0.61,0.60$ and 0.56 at TR $=0.7 \mathrm{~s}$ (for TM: $0.03,0.042$ and $0.1 \mathrm{~s}$ in both cases, respectively).

Figure 5.8 shows, on the other hand, the STEAM SE signal obtained from an ex vivo human brain sample acquired at two different TR's ( 0.35 and $1.00 \mathrm{~s}$ ) and several TM's, equally scaled. Figure 5.8A shows how the image intensity and contrast varies as a function of TM for several $\mathrm{T}_{1}$ values from different substrates (i.e. white matter, grey matter, and fluid). The long $\mathrm{T}_{1}$ attenuation effect, stronger for the shorter TR $(0.35 \mathrm{~s})$ than for the longer TR $(0.7 \mathrm{~s})$, which was demonstrated in Figure 5.6, is appreciable. At equal TM (e.g. $0.12 \mathrm{~s}$ ) the signal from long $\mathrm{T}_{1}$ 's (i.e. from the solution) is recovered at longer TR. In contrast, the signals from white matter (WM) and grey matter (GM) show little signal variation at different TR with the same TM. As shown in Figure 5.8B (and similarly shown in 5.6), the signal from GM is higher because of its shorter $\mathrm{T}_{1}(\sim 0.3 \mathrm{~s}$ as estimated in Chapter 3$)$ in comparison to $\mathrm{WM}(\sim 0.5 \mathrm{~s})$ and solution $(\sim 4.0 \mathrm{~s})^{4}$. The qualitative similarity between the modelled signal from Equation 5.10 (and shown in Figure 5.6) and the acquired data is also clear.

A more quantitative comparison between the modelled and experimental SE ex vivo brain signals, including the observed attenuation effects, is shown in 5.9. By comparing the SE signal ratios between WM/GM and solution/GM from the modelled (dashed lines) and acquired MR data (solid lines), shows both a contrast of interest (WM/GM) and a measure of fluid attenuation (Fluid/GM). Besides that, it also ensures that the effects of other signal components in the experimental data (e.g. Tx-Rx profiles) are minimised. For both ratios, at both TR's, the correspondence between the modelled and experimental data is high, except when TM approaches TR (i.e. for short effective TR) This points out that one of the assumptions (Equation 5.4) is not fulfilled in the experimental data.

\footnotetext{
${ }^{4} \mathrm{GM}$ also has higher $\mathrm{PD}$ and $\mathrm{T}_{2}$ values, as shown in Chapter 3 , in comparison to WM.
} 


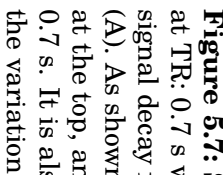

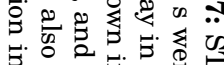

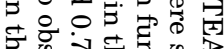

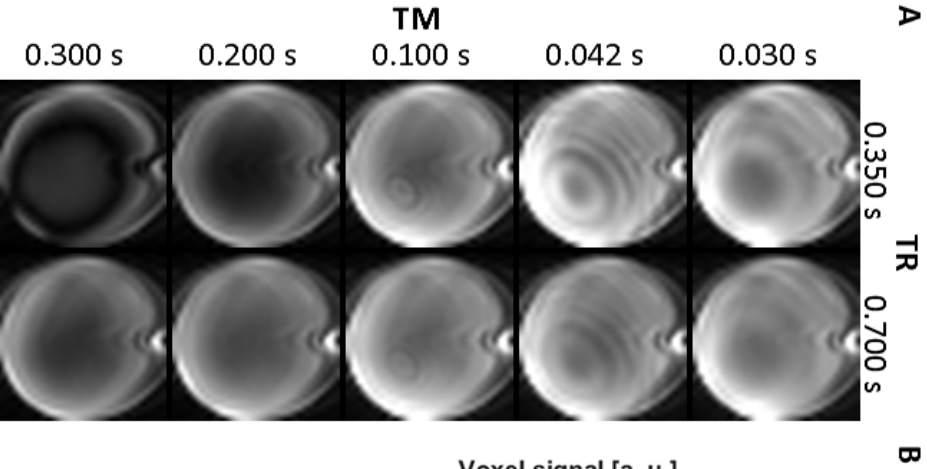

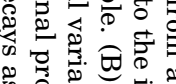

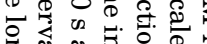

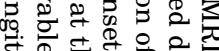

टे 0 के

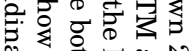

ข

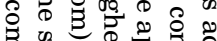

定

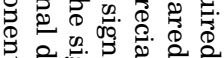

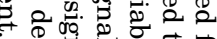
象 $\Omega$

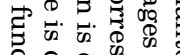

골 율융 유

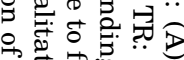
茟寻品 o o

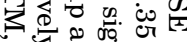
级蕰包 룰을 के 률

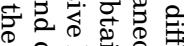

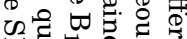
खा

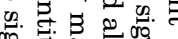
黄

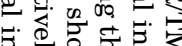
这完局

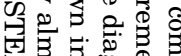

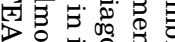
设

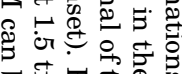

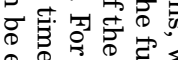
ब

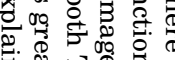

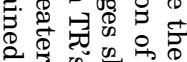

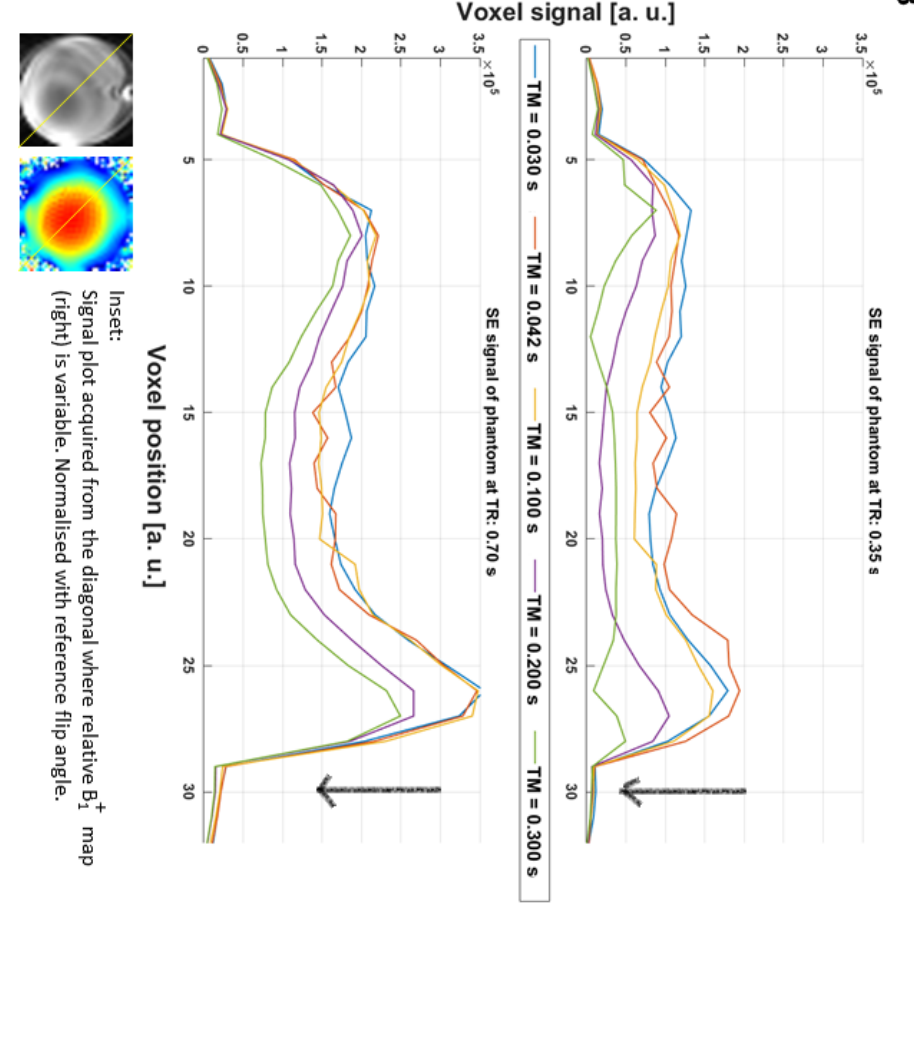

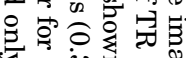

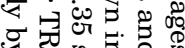




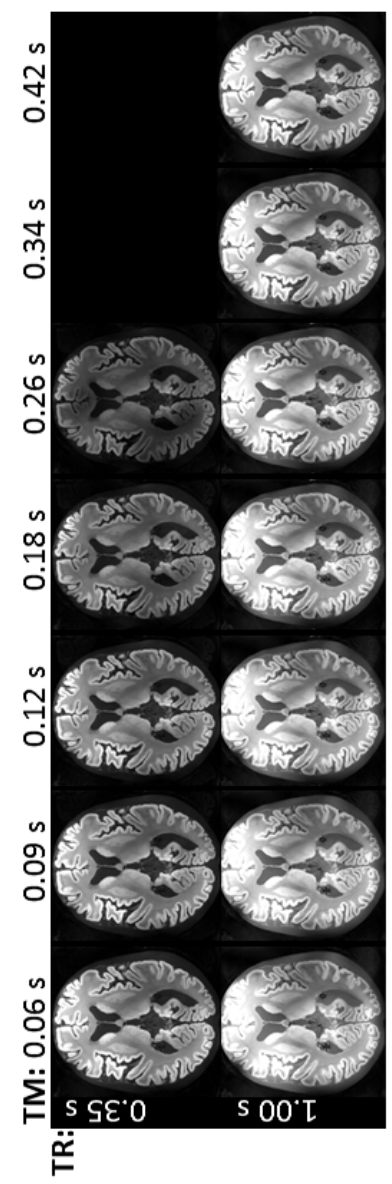

$\varangle$

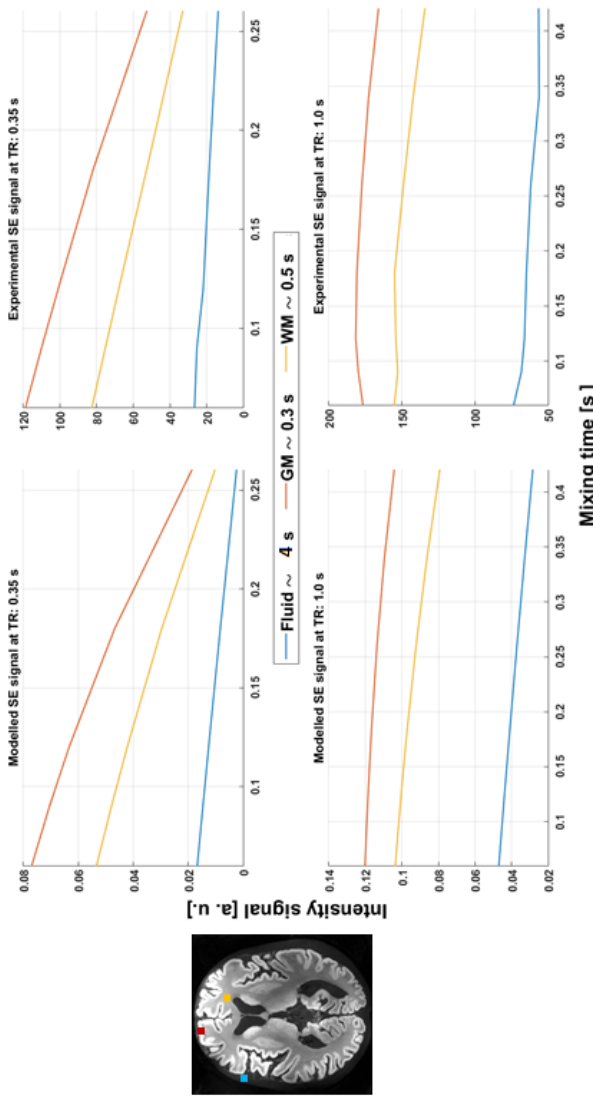

$\infty$

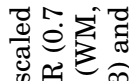

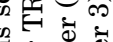

ปี

过 임

그으워

댕 类

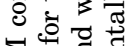

¿ శ శ

돈 क्षे

$+\widehat{x}$

क $10 \sum^{2}$

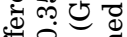

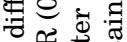

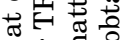

\% क्ष द्व

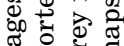

दू क्ष के है

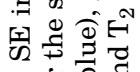

造 ฮี

겅 을

니용

잉 \&

क 要吉

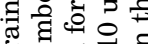

흐웜엉 웅

○ $\mathrm{H}^{-1}$ 의

5 我赵

$x$ 으응 중

0 을

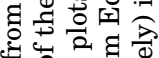

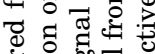

$\exists$.

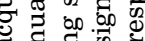

क क्ञ

๑

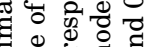

를 व

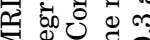

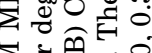

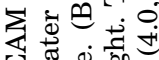

될 क्ष

政芆

$\ddot{0}$ 可

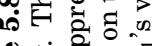

文各合

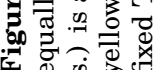




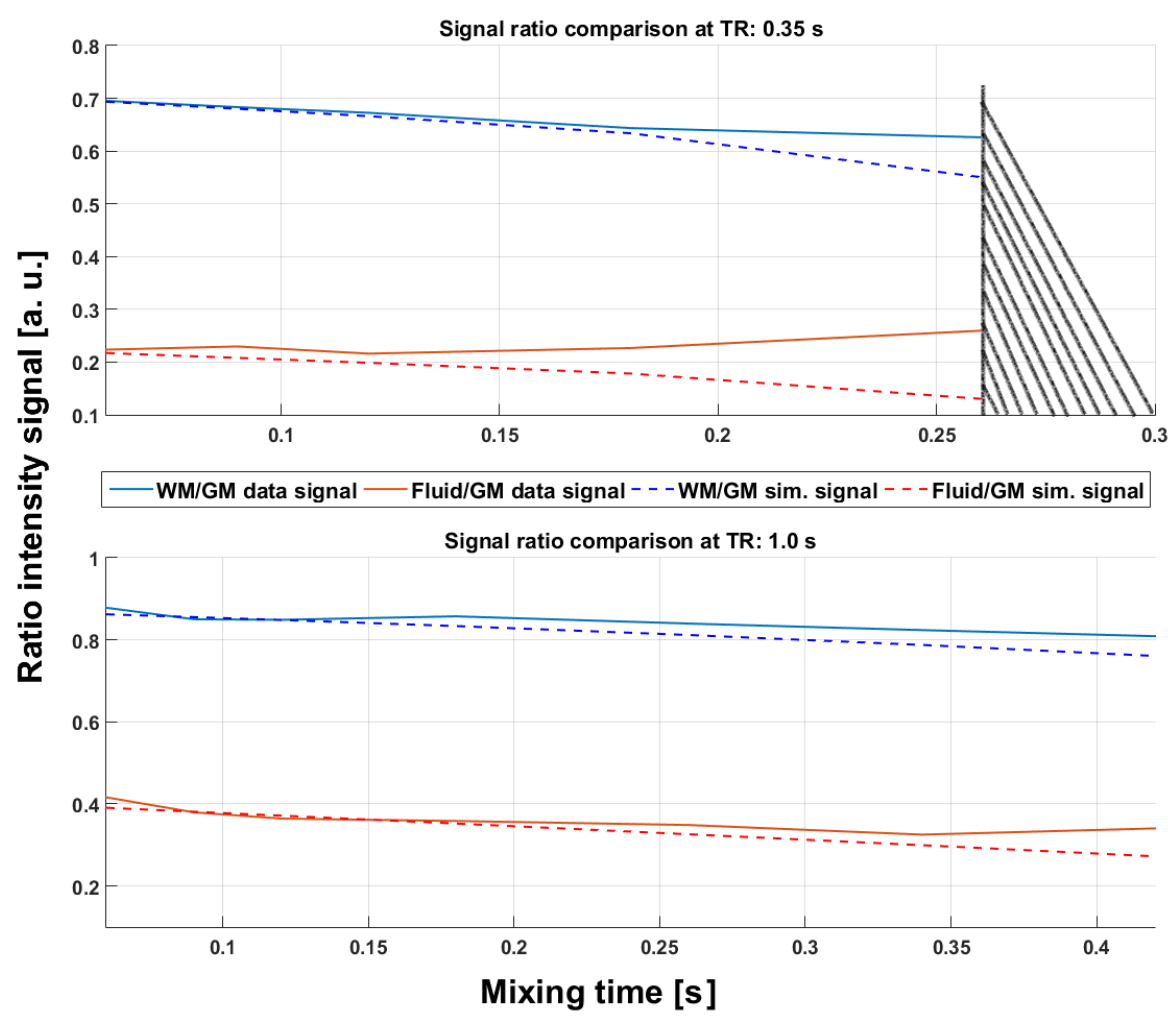

Figure 5.9: $\mathrm{SE}$ signal ratios both modelled by Equation 5.1 with $\mathrm{M}_{\mathrm{z}}$ defined by 5.10 (dashed lines), and measured experimentally with ex vivo data from Figure 5.8 (solid lines). SE signal ratios are shown for WM/GM (blue) and solution/GM (red) for TR: $0.35 \mathrm{~s}$ (top) and $0.7 \mathrm{~s}$ (bottom). The shadowed area represents the restriction of TM due to TR.

\subsection{Discussion}

This work aimed to study in detail the contribution of the longitudinal magnetisation component in the SE and STE signals when the STEAM sequence reaches a steady-state regime (i.e. TM, TR $<\mathrm{T}_{1}$ ). Even though this component was previously introduced (e.g. in references [7, 238]), it became necessary to obtain a complete analytical expression in which it can be broadly explored and studied. These analyses allowed not only to study this component as a function of TR, TM and $\mathrm{T}_{1}$ as previously estab- 
lished; but it was also possible to explore it in case of variable flip angles and possible remnant SE signal contribution. The addition of this component to the transverse SE and STE signal equations helped to understand in deeper detail the variation of these signals under several scenarios and, at the same time, it established a precedent regarding the expectations on the SE and STE signals in empirical observations in restricted regimes. Given the fact that the experimental signal behaviour can be explained by the signal model shown in this work, makes it possible to explain no intuitive empirical results (e.g. SE signal decay as a function of TM). Or, even better, giving insights into the possibilities of implementing other contrast mechanisms like fluid-attenuated, or dark-blood, STEAM imaging.

\subsubsection{A steady-state signal in the STEAM sequence}

To acquire an analytical expression for $\mathrm{M}_{\mathrm{z}}$, it was necessary to solve the Bloch equation for STEAM under two assumptions: where there is no residual transverse magnetisation after $\mathrm{TR}$, and the sequence reached an steady-state regime immediately after the first TR. From this solution, two equations were obtained which represent the cases where a remnant SE signal contributes to this component (referred as unspoiled SE, Equation 5.10 and 5.13 for $\alpha=\pi / 2 \mathrm{rad}$ ) or it is fully suppressed (spoiled SE, Equation 5.12 and 5.14 for $\alpha=\pi / 2$ rad). Figure 5.7 showed how the proposed $\mathrm{M}_{\mathrm{z}}$ models differ from each other. The difference between both is highest at the short TM and TR regime (TM, TR « $\mathrm{T}_{1}$ ), and with $\mathrm{TM}$ shorter than $\mathrm{T}_{2}$ (fixed at $0.040 \mathrm{~s}$ ). This difference is explained by the $\mathrm{E}_{2}(\mathrm{TM})$ component in Equation 5.13. In the time regime where TM becomes higher than $\mathrm{T}_{2}$, both equations become indistinguishable and can be used to describe the signal equally. Therefore, without losing generality, the following results were performed using equations 5.10 and 5.13.

\subsubsection{Steady-state signal dependencies and total signal behaviour}

Figures 5.3 and 5.4 showed the STE signal dependency, with the included steady-state component, with respect to TM, TR and flip angles. To generalise the results, it was necessary to normalise all the timing parameters to $\mathrm{T}_{1}$. Figure 5.3 clearly shows the contribution of the RF pulses to the STE signal behaviour: the excitation flip angle only modulates the total signal available in the STEAM sequence, while the storing and recalling 
RF pulses not only scale but are also more sensitive to TR-TM values. In the SE signal (not shown), on the other hand, the excitation flip angle contributes in the same way but in the storing RF pulse, even if it shows a similar TM-TR dependency as in STE, the signal increases with higher flip angle. Figure 5.4 shows the STE signal (expressed in units of SNR-eff) only dependent on TM-TR (in the TM-TR space) while fixing the flip angles at $\pi / 2 \mathrm{rad}$ (where it is maximum). On a global scale, the highest SNR-eff is achieved in the shortest TM with a TR close to 1.4 times $\mathrm{T}_{1}$. It is also shown that it is possible to find a set of TM-TR values where a maximum SNR-eff (Figure 5.4B) can be achieved. This would allow defining STEAM acquisitions protocols with optimised SNR-eff by using the obtained linear relationship defined in Equation 5.15. As an example, Table 5.2 shows several TM-TR values required to obtain STE images in ex vivo and in vivo human brains at $9.4 \mathrm{~T}$ and $7 \mathrm{~T}$ respectively with the highest SNR-eff.

\begin{tabular}{|l|c|c|}
\hline TM/Desired TR & Ex vivo WM & In vivo WM \\
& $9.4 \mathrm{~T}-\mathrm{T}_{1}: 0.3 \mathrm{~s}$ & $7 \mathrm{~T}-\mathrm{T}_{1}: 1.1 \mathrm{~s}$ \\
\hline $0.5 \mathrm{~T}_{1} / 2.2 \mathrm{~T}_{1}$ & $0.15 \mathrm{~s} / 0.66 \mathrm{~s}$ & $0.55 \mathrm{~s} / 2.42 \mathrm{~s}$ \\
$1.0 \mathrm{~T}_{1} / 3 \mathrm{~T}_{1}$ & $0.3 \mathrm{~s} / 0.9 \mathrm{~s}$ & $1.1 \mathrm{~s} / 3.3 \mathrm{~s}$ \\
$2.0 \mathrm{~T}_{1} / 5 \mathrm{~T}_{1}$ & $0.6 \mathrm{~s} / 1.5 \mathrm{~s}$ & $2.2 \mathrm{~s} / 5.5 \mathrm{~s}$ \\
\hline
\end{tabular}

Table 5.2: TR required for a predefined TM to achieve the highest SNR efficiency for ex vivo and in vivo human brain MR studies. The desired TR was obtained by using Equation 5.15.

On the other hand, it was important to examine how the different components (longitudinal and transverse) in the STE signal modelling are modulated by TR and TM. Figure 5.5 (top) showed how the longitudinal component is highly dependent on TR and almost null on TM. Moreover, for all $\mathrm{T}_{1}$ values, the intensity of this component increases by TR as well. Therefore, even if the effective TR is defined to be TR/TE/TM dependent (which explains the angled isocurves), the relation between $T R / T_{1}$ is predominant. The transverse component, on the other hand, is only TM dependent as expected (which explains the vertical isocurves in 5.5 middle). Thus, the combined dependencies of TR and TM permits the modulation of the STE signal at different $T_{1}$ 's to achieve desired contrasts or attenuation. For example, for short $\mathrm{T}_{1}$ values, the longitudinal component gets recovered really fast (or already fully) at really short TR values $\left(\sim 2 \mathrm{~T}_{1}\right)$ but it is barely recovered for long $\mathrm{T}_{1}$ 's. At the same time, in the opposite scenario, the transverse component gets attenuated really fast by TM in short $\mathrm{T}_{1}$ 's but slowly decays for long $\mathrm{T}_{1}$ 's. Therefore, as shown in Figure 5.6, it is possible 
to obtain TR-TM combinations where the contrast between different $T_{1}$ 's is minimised or maximised. Examples of this STEAM contrast benefit is shown in Figure 5.10 for GM and solution, where the contrast is almost minimum; as well as the almost full attenuation of fluid in the short TR regime, while GM and WM signals become contrast-enhanced.

\subsubsection{Experimental data comparison with signal modelling}

Figures 5.7 and 5.8 showed the experimental signal behaviour of the SE signal at different TR/TM/flip angle combinations. Figure 5.7 showed clearly how, for a homogeneous specimen (with an expected constant $T_{1}$ ), the signal varies with timing and flip angle parameters. The SE signal decays as a function of TM, which is not expected if it is explained by the transverse component formulation only. Then, this variation must come from the longitudinal component dependency on this parameter. Using a qualitative comparison between images, shows an increment of the signal at increasing TR, as indicated in Figure 5.5 (top). From this qualitative comparison between experimental signal behaviour and modelling, it was possible to study in more detail how accurately the proposed signal formulation (Equation 5.10) can model the experimental SE signal from STEAM acquisitions in a more complex system like an ex vivo brain specimen. Figure $5.8 \mathrm{~A}$ showed similar signal changes as in phantom: increasing signal with increased TR at constant TM and, at the same time, the signal decays with increased TM. The increasing TR clearly benefits the signal coming from the solution, because it possesses the longest $\mathrm{T}_{1}$ in comparison to the biological structures in the brain specimen. As shown in Figure 5.6, it is clear how the combination between TR-TM gives a signal advantage for a specific $\mathrm{T}_{1}$ value, like in this case for GM. Figure 5.8B shows an explicit comparison between the simulated signal (first column) and the signal from the acquired data (second column). Qualitatively, the signal behaviour of those biological structures are identical to those proposed by the model. Figure 5.9 takes the next step and compares the model and the experimental data by taking the ratios between WM/GM and solution/GM. It shows a strong correlation of the model with the experimental data, especially for the WM/GM ratio in which the highest difference for the longest TM is almost $11 \%$ in the short TR regime and 5.6\% in the long TR regime. The bifurcation in the similarity between the simulated and experimental data in the long TM's can be explained with the break of one of the conditions 
(either the steady-state regime has not been achieved or the remaining transverse magnetisation is not completely spoiled, Equation 5.4) in the experimental data. Therefore, the use of the proposed $M_{z}$ components in the SE signal equation can represent adequately the behaviour of the SE signal decay and by extension to STE signal. It is important to remark, again, that demonstrating this point using STE images instead could be more complicated due to the high dependence of this signal to TM (i.e. in the longitudinal component, in the relaxation transverse component and in the diffusion component) as shown in Figure 5.10.

\subsection{Conclusion}

In this work, full SE and STE signal equations from the STEAM sequence were obtained. It was modelled for cases of unspoiled and spoiled SE signal, timing parameters (i.e. TR-TM- $T_{1}$ ) and flip angles. Using this component for a full STE signal modelling, it was possible to obtain a relationship between TM-TR for achieving the highest SNR-eff possible and maximising the contrast of a specific $\mathrm{T}_{1}$ by equalising the signal coming from other $\mathrm{T}_{1}$ components. The corroboration of this modelling was performed by comparing it with the SE signal behaviour from experimental data. High correspondence between the experimental and modelling signal for cases of homogeneous and inhomogeneous specimens were obtained. With this complete signal modelling, it is possible to understand the SE and STE signals for different $\mathrm{T}_{1}$ 's in a broader range of times (TR, TM) and flip angles. Besides, in the future the option of performing relaxometry with variable TR and diffusion with variable TM-TR analysis can be tested by using both signals without using the ratio (if available). For instance, it can be ideal for microstructure diffusion analysis, where the SE and STE signals can be obtained simultaneously in STEAM acquisition at different diffusion times (therefore, $b$-values), where the SE diffusion signal can be used for short diffusion time regimes while the STE diffusion signal can be used for long diffusion time regimes. 


\subsection{Supplementary material}

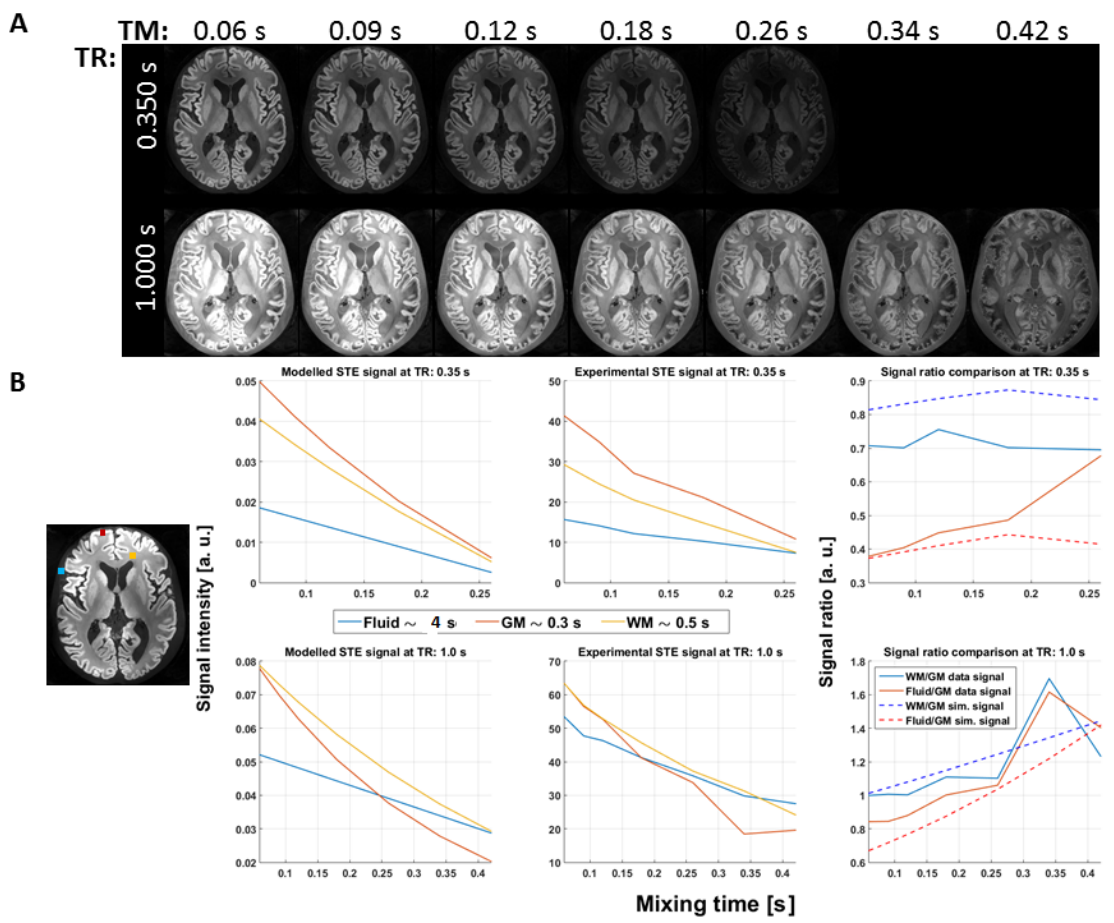

Figure 5.10: STEAM MRI images acquired from ex vivo brain specimen data: (A) STE images at different TR/TM combinations. Signal increment in function of TR is shown and simultaneous signal decay in function of TM are appreciable. It is noticeable how the signal decay from solution is minimum in the first situation, explained by its long $\mathrm{T}_{1}$ value. (B) Corresponding signal plots obtained for three different positions: solution (blue), grey matter (GM, red) and white matter (WM, yellow). The modelled signal from Equation 5.10 is shown along the first column by using as input the $\mathrm{B}_{1}{ }^{+}$and $\mathrm{T}_{2}$ maps obtained experimentally (Chapter 3 ) and fixed $\mathrm{T}_{1}$ 's values (4.0, 0.3 and $0.5 \mathrm{~s}$ respectively). The corresponding experimental signal decay is shown in the second column. It is clear that the intersection between signals shown in Figure 5.6 could be replicated in the STE signal. The third column shows the signal decay ratios for WM/GM (blue) and solution/GM (red) from the experimental data (solid lines) and modelled data (dashed lines). In comparison to Figure 5.9, the correspondence is not as high, since the diffusion component is not added to the signal modelling. 



\section{MESMERISED: Super-accelerated 7 T STEAM imaging for quantitative multi-contrast and diffusion MRI ${ }^{1}$}

\footnotetext{
${ }^{1}$ From the pre-printed article: "Fritz et al. MESMERISED: Super-accelerated $7 T$ STEAM imaging for quantitative multi-contrast and diffusion MRI. BioArXiv, 2020, DOI:https://doi.org/10.1101/2020.05.15.098269 " and under review. From the accepted abstracts: "Fritz et al. MESMERISED: Super-accelerated 7T STEAM imaging for quantitative T1 and diffusion MRI. ISMRM 27th Annual Meeting and Exhibition, Montreal, 2019" (accepted as Oral Presentation and awarded with Summa cum Laude, and the Ultra-High Field Study Group 1 Abstract Award), and "Fritz et al. MESMERISED: Super-accelerated 7T STEAM imaging for quantitative T1w MRI. ISMRM Benelux Chapter 11th Annual Meeting and Exhibition, Leiden, 2019" (accepted as Poster)
} 


\section{Abstract}

There is an increasing interest in quantitative imaging of $T_{1}, T_{2}$ and diffusion contrast in the brain due to greater robustness against bias fields and artefacts, as well as better biophysical interpretability in terms of microstructure. However, acquisition time constraints are a challenge, particularly when multiple quantitative contrasts are desired and when extensive sampling of diffusion directions, high b-values or long diffusion times is needed for multi-compartment microstructure modelling. Although ultrahigh fields of $7 \mathrm{~T}$ and above have desirable properties for many MR modalities, the shortening $\mathrm{T}_{2}$ and the high specific absorption rate (SAR) of inversion and refocusing pulses bring great challenges to quantitative $T_{1}$, $\mathrm{T}_{2}$ and diffusion imaging. Here, we present the MESMERISED sequence (Multiplexed Echo Shifted Multiband Excited and Recalled Imaging of STEAM Encoded Diffusion). MESMERISED removes the dead time in stimulated acquisition mode (STEAM) imaging by an echo-shifting mechanism. The echo-shifting (ES) factor is independent of MB acceleration and allows for very high multiplicative ESxMB acceleration factors, particularly under moderate and long mixing times. This results in high duty cycle and time efficiency at $7 \mathrm{~T}$, particularly for quantitative $\mathrm{T}_{1}$ and diffusion imaging, while also retaining the capacity to perform quantitative $T_{2}$ and $B_{1}$ mapping. We demonstrate the super-acceleration of MESMERISED for wholebrain $\mathrm{T}_{1}$ relaxometry with total acceleration factors up to 36 at $1.8 \mathrm{~mm}$ isotropic resolution, and up to 54 at $1.25 \mathrm{~mm}$ resolution $\mathrm{qT}_{1}$ imaging, corresponding to a $6 \mathrm{x}$ and $9 \mathrm{x}$ speed-up, respectively, compared to MB-only accelerated acquisitions. We demonstrate quantitative $T_{2}$ and $B_{1}$ mapping to illustrate multi-contrast mapping with the same MESMERISED sequence and the same readout train. Finally, we demonstrate highly efficient diffusion MRI with high b-values and long diffusion times in two separate cases. First, we show that super-accelerated multi-shell diffusion acquisitions with 370 whole-brain diffusion volumes over 8 b-value shells up to $b=7000 \mathrm{~s} / \mathrm{mm}^{2}$ can be generated at $2 \mathrm{~mm}$ isotropic in under 8 minutes, a data rate of almost a volume per second, or at $1.8 \mathrm{~mm}$ isotropic in under 11 minutes. Second, we demonstrate a time-efficient sampling of different diffusion times with $1.8 \mathrm{~mm}$ isotropic diffusion data acquired at four diffusion times up to $290 \mathrm{~ms}$, which supports both DTI and DKI at each diffusion time. MESMERISED extends possibilities to efficiently probe $\mathrm{T}_{1}, \mathrm{~T}_{2}$ and diffusion contrast for multi-component modelling of tissue microstructure. 


\subsection{Introduction}

Magnetic resonance imaging (MRI) derives most of its versatility as an imaging technique from being able to weight images towards a multitude of contrasts, prominently among which are $\mathrm{T}_{1}, \mathrm{~T}_{2} / \mathrm{T}_{2}{ }^{*}$ and diffusion contrasts. Recently, there is an increasing interest in quantitative imaging of these contrasts for two main reasons. First, quantitative $\mathrm{T}_{1}, \mathrm{~T}_{2}$ and diffusion images possess greater robustness against bias fields and artefacts, since the effects of spatially slowly varying radiofrequency (RF) transmit and receive fields are mostly removed. Second, quantitative images have better biophysical interpretability, since they have interpretable physical units. This makes them a better proxy for biological microstructure and phenomena, such as myelin (e.g. in references [43, 139, 225]), iron (e.g. in references [225, 196, 89]), axonal density (e.g. in references [8, 237]) or coarse-graining due to random axonal packing (e.g. in reference [157]). However, since quantitative MRI (qMRI) requires the acquisition of many volumes, from which the quantitative image is then derived, long acquisition times are a challenge, particularly when multiple quantitative contrasts are desired.

Acquisition time constraints are particularly pressing for diffusion MRI (dMRI). The signal varies with the orientation, strength and diffusion time encoded by diffusion gradients, making diffusion contrast inherently multidimensional. Moreover, recent developments in biophysical microstructure quantification with dMRI require sampling more of the multi-dimensional contrast space than with older methods. The conventional method for the analysis of white matter in dMRI imaging is the tensor model in Diffusion Tensor Imaging (DTI; $[18,19,165])$. Although a DTI derived index variable such as Fractional Anisotropy (FA) is sensitive, it is also unspecific, since differences in FA can reflect different axonal properties such as axon density, diameter distribution and myelination [11, 10, 23, 62, 117]. Therefore, advances in diffusion microstructure modelling have aimed at greater specificity than DTI in relating the dMRI signal to the underlying cellular microstructure [5, 13, 8, 237, 11, 82, 109, 161, 62, 61, 12]. Models such as the Neurite Orientation Dispersion and Density Imaging model (NODDI; [237]), the Combined Hindered And Restricted Model of Diffusion (CHARMED; [8]), and the White Matter Tract Integrity model (WMTI; [82]) can provide measures specific to fibre density and orientation dispersion. These require the acquisition of a large number of diffusion directions over multiple $b$-values, sometimes extending to b-values as high 
as $6000-8000 \mathrm{~s} / \mathrm{mm}^{2}$, leading to acquisition times of $10-20$ minutes or longer. Sensitivity can additionally be gained to axonal diameter distributions [5, 12, 236, 63], water-exchange over cell membranes [132, 154] and coarse-graining effects $[39,157]$, but this requires the acquisition of a large number of b-values and diffusion times extending to $200-800 \mathrm{~ms}$, often leading to acquisition times of $30-60 \mathrm{~min}$.

Developments in echo-planar imaging (EPI) acquisition have allowed significant speed-up in the acquisition of volume time series weighted for $\mathrm{T}_{1}$, $\mathrm{T}_{2} / \mathrm{T}_{2}{ }^{*}$ or diffusion. Prominently among these is multiband (MB) or simultaneous multi-slice (SMS) acquisition [80, 148, 182], which allows 2- to 4-fold speed-up for high flip angle applications at the expense of a higher specific absorption rate (SAR). However, even with MB acceleration, quantitative multi-contrast studies that require extensive diffusion MRI, as well as quantitative mapping of $\mathrm{T}_{1}$ and/or $\mathrm{T}_{2}$, are facing prohibitively long acquisition times.

In the domain of high spatial resolution imaging, ultra-high fields (UHF) of 7 Tesla $(7 \mathrm{~T})$ and above have enabled efficient high spatial resolution mapping of quantitative $\mathrm{T}_{1}$ (e.g. in references $[142,160]$ ), $\mathrm{T}_{2}{ }^{*}$ (e.g. in reference [89]) and diffusion (e.g. see reference [220]). In this work, our main aim was to establish whether the high signal-to-noise ratio (SNR) available at $7 \mathrm{~T}$ could be used for highly time-efficient quantitative multicontrast acquisition at moderate resolutions, particularly when including dMRI at high b-values or long diffusion times. Although UHF has desirable properties for many MR modalities, the shortening of $\mathrm{T}_{2}$ and the high SAR load of inversion and refocusing pulses bring challenges to efficient quantitative $T_{1}, T_{2}$ and diffusion imaging. Diffusion MRI sequences such as pulsed-gradient spin-echo (PGSE; e.g. in reference [220]) and steadystate free precession (SSFP; e.g. in reference [136]) have been optimised at $7 \mathrm{~T}$ to mitigate some of these limitations. However, they remain limited to MB acceleration factors of 2 to 4 [75, 220, 232], with SAR as the main limiting factor.

Therefore, we investigated the possibilities of the stimulated echo acquisition mode (STEAM) sequence [85]. The STEAM sequence has some attractive advantages for multi-contrast MRI at UHF. First, it is highly multicontrast capable, being able to provide quantitative $\mathrm{T}_{1}\left(\mathrm{q} \mathrm{T}_{1}\right)$ contrast (by relaxometry on the mixing time, $\mathrm{TM}$ ), quantitative $\mathrm{T}_{2}\left(\mathrm{qT}_{2}\right)$ contrast (by relaxometry on the echo time, $\mathrm{TE}$ ), transmit $\left(\mathrm{B}_{1}{ }^{+}\right)$field mapping, and quantitative diffusion contrast. Furthermore, both b-value and diffusion time parameters in diffusion MRI with STEAM can be probed at the high values desirable for many diffusion microstructure applications. Second, both a 
spin-echo (SE) and stimulated echo (STE) signal can be independently acquired after a single excitation pulse, enhancing the capacity for efficient multi-contrast acquisition. Third, STEAM is highly efficient in SAR given its preferred $90^{\circ}-90^{\circ}-90^{\circ}$ flip angle scheme. It has $\sim 60 \%$ of the SAR of a comparable $90^{\circ}-180^{\circ}$ spin-echo sequence and $\sim 33 \%$ of the SAR of a comparable $180^{\circ}-90^{\circ}-180^{\circ}$ inversion-prepared spin-echo sequence (assuming similar slice-selection and a quadratic SAR/flip angle relationship). This also means that applying multiband pulses for SMS imaging is easier to apply for high MB factors in STEAM since SAR increases linearly with MB factor. However, the STEAM sequence also has inherent disadvantages. Both the SE and STE signals have only $50 \%$ of the signal level and signal to noise ratio (SNR), compared to the SE from a $90^{\circ}-180^{\circ}$ spin-echo sequence. Moreover, there is a considerable dead time in STEAM (Figure 6.1A), especially when moderate to long mixing times (useful for $\mathrm{T}_{1}$-weighting and probing of long diffusion times) are required, which leads to a low SNR per unit time efficiency.

Here, we present the MESMERISED sequence (Multiplexed Echo Shifted Multiband Excited and Recalled Imaging of STEAM Encoded Diffusion). MESMERISED removes the dead time in stimulated acquisition mode (STEAM) imaging by an echo-shifting mechanism. The echo-shift (ES) factor is independent of $\mathrm{MB}$ acceleration and allows for very high multiplicative ESxMB acceleration factors, particularly under moderate and long TM's. This results in a high duty cycle and high time efficiency at 7 $\mathrm{T}$, particularly for quantitative $\mathrm{T}_{1}$ and diffusion imaging. We demonstrate the super-acceleration of MESMERISED for whole-brain $\mathrm{T}_{1}$ relaxometry with total acceleration factors up to 36 for $1.8 \mathrm{~mm}$ resolution and up to 54 for $1.25 \mathrm{~mm}$ resolution $\mathrm{qT}_{1}$ imaging. We then demonstrate quantitative $\mathrm{T}_{2}$ and $\mathrm{B}_{1}{ }^{+}$mapping to illustrate multi-contrast mapping with the same MESMERISED sequence. Finally, we demonstrate highly efficient diffusion MRI with high b-values and long diffusion times in two cases: First, we show super-accelerated multi-shell diffusion acquisitions with 370 wholebrain diffusion volumes over $8 \mathrm{~b}$-value shells up to $\mathrm{b}=7000 \mathrm{~s} / \mathrm{mm}^{2}$. Second, we demonstrate a time-efficient sampling of different diffusion times with $1.8 \mathrm{~mm}$ isotropic diffusion data acquired at four diffusion times up to 290 ms. 


\subsection{Theory}

The MESMERISED sequence is based on the standard (diffusion-weighted) STEAM sequence (Figure 6.1A). The STEAM pulse sequence uses three radiofrequency (RF) pulses: excitation (Exc), storing (Sto) and recalling (Rec) pulses, which can create several spin echoes (SE's) and a stimulated echo (STE), and was first introduced by Frahm and colleagues [85]. In general terms, the excitation pulse places the magnetisation into the transverse plane where it undergoes $\mathrm{T}_{2}$ decay. Subsequently, the storing pulse places ('stores') part of the total magnetisation along the longitudinal axis where it is subject to $\mathrm{T}_{1}$ decay during the mixing time (TM). The remaining part of the magnetisation forms the primary SE signal. Finally, the recalling pulse places ('recalls') the stored magnetisation to the transverse plane where it once again undergoes $\mathrm{T}_{2}$ decay until the STE is formed. This sequence of pulses can create three more SE signals (the second, third and fourth spin-echo) after the STE, which are the consequence of the free induction decay (FID) signal generated by the storing and recalling pulses. We focus on the first or primary SE signal in the remainder of this work and refer to it simply as 'the SE' hereafter. Whereas the SE undergoes only by $\mathrm{T}_{2}$ decay, the STE signal undergoes both $\mathrm{T}_{1}$ and $\mathrm{T}_{2}$ decay, because the signal is obtained from spins stored along the longitudinal axis for the duration of the TM, during which there is $\mathrm{T}_{1}$ relaxation. Furthermore, both echoes (SE and STE) share the transverse magnetisation created by the excitation pulse and therefore split the total excited signal, which becomes evenly distributed between SE and STE when the storing pulse has a flip angle of $90^{\circ}$.

MESMERISED removes the dead time in STEAM by shifting Exc-Sto pulse pairs and SE readout for one or more MB slice groups into the dead time of earlier slice groups (Figure 6.1B). We refer to this as echo-shifting, by analogy to sequences that shift sequence elements to achieve echo times longer than their repetition time (TR) in gradient-echo or steady-state free precession sequences (c.f. see reference [74, 90]). However, we note the shifting mechanism here is fundamentally different and does not achieve TE > TR. The echo-shifting in MESMERISED temporally interleaves and multiplexes the contrast evolution of several slices or MB slice-groups. The stored longitudinal magnetisation of earlier Exc-Sto and SE readout sequence blocks ( $\mathrm{SB}^{\mathrm{A}}$ blocks) evolves while later $\mathrm{SB}^{\mathrm{A}}$ blocks are executed and SE's are acquired. Subsequently, STE's are recalled and read-out in a sequence of $\mathrm{SB}^{\mathrm{B}}$ blocks (Figure 6.1B), placed at time TM to their corre- 
sponding $\mathrm{SB}^{\mathrm{A}}$ blocks. We refer to the number of echo-shifted slice groups as the echo-shift factor (ES factor). The echo-shifting is independent of MB acceleration and allows for very high multiplicative ESxMB acceleration factors, particularly under moderate and long TM's. This results in full duty cycle and high time efficiency, particularly for quantitative $\mathrm{T}_{1}$ and diffusion imaging.

For instance, $\mathrm{T}_{1}$ relaxometry with STEAM requires a range of TM's acquired under the same TR and TE. Whereas total acquisition time of $\mathrm{T}_{1}$ relaxometry with MB-STEAM would be determined by the TR of the longest TM, acquisition time of $\mathrm{T}_{1}$ relaxometry with MESMERISED is limited by the TR of shortest possible TM. All TM's required for accurately fitting an exponential decay model can be acquired at the full duty cycle and a short TR. Essentially, in MESMERISED a different choice of ES factor determines a simple reordering of the same sequence blocks and enable a different mixing time to be acquired at the same minimised TR (see Supplementary Figure 6.15).

For diffusion imaging, monopolar diffusion gradient pairs are inserted symmetrically around the storing and recalling pulses. Although only the first (before the storing pulse) and fourth (after the recalling pulse) gradients are needed for diffusion-weighting of the STE signal, there are several advantages to a double symmetric placement of four gradients. First, these also serve as crusher gradients at low amplitude and duration in non-diffusion-weighted imaging, helping to remove unwanted echo pathways. Second, the first pair of gradients create a pulsed-gradient spin-echo (PGSE) acquisition in the $\mathrm{SB}^{\mathrm{A}}$ block, with the corresponding PGSE diffusion contrast in the SE. Third, the second and third gradient act as spoilers during the mixing time for unwanted (secondary and tertiary) spin-echo pathways. Additionally, this spoiler action is enhanced in MESMERISED, since the gradients of echo-shifted blocks add to the total spoiler-moment during TM. MESMERISED can acquire diffusion-weighted signals at long diffusion times and high b-values in its STE signal, at relatively low duty cycle demands on the gradients. Besides, it allows highly accelerated probing of multiple diffusion times at the same $\mathrm{TR}$ and $\mathrm{TE}$ as for $\mathrm{T}_{1}$ relaxometry explained above. Finally, as a STEAM variant with both a SE and STE readout, MESMERISED can acquire both a low b-value and short diffusion time (in its diffusion-weighted SE's) and a high b-value and long diffusion time (in its diffusion-weighted STE's) in a single TR. 


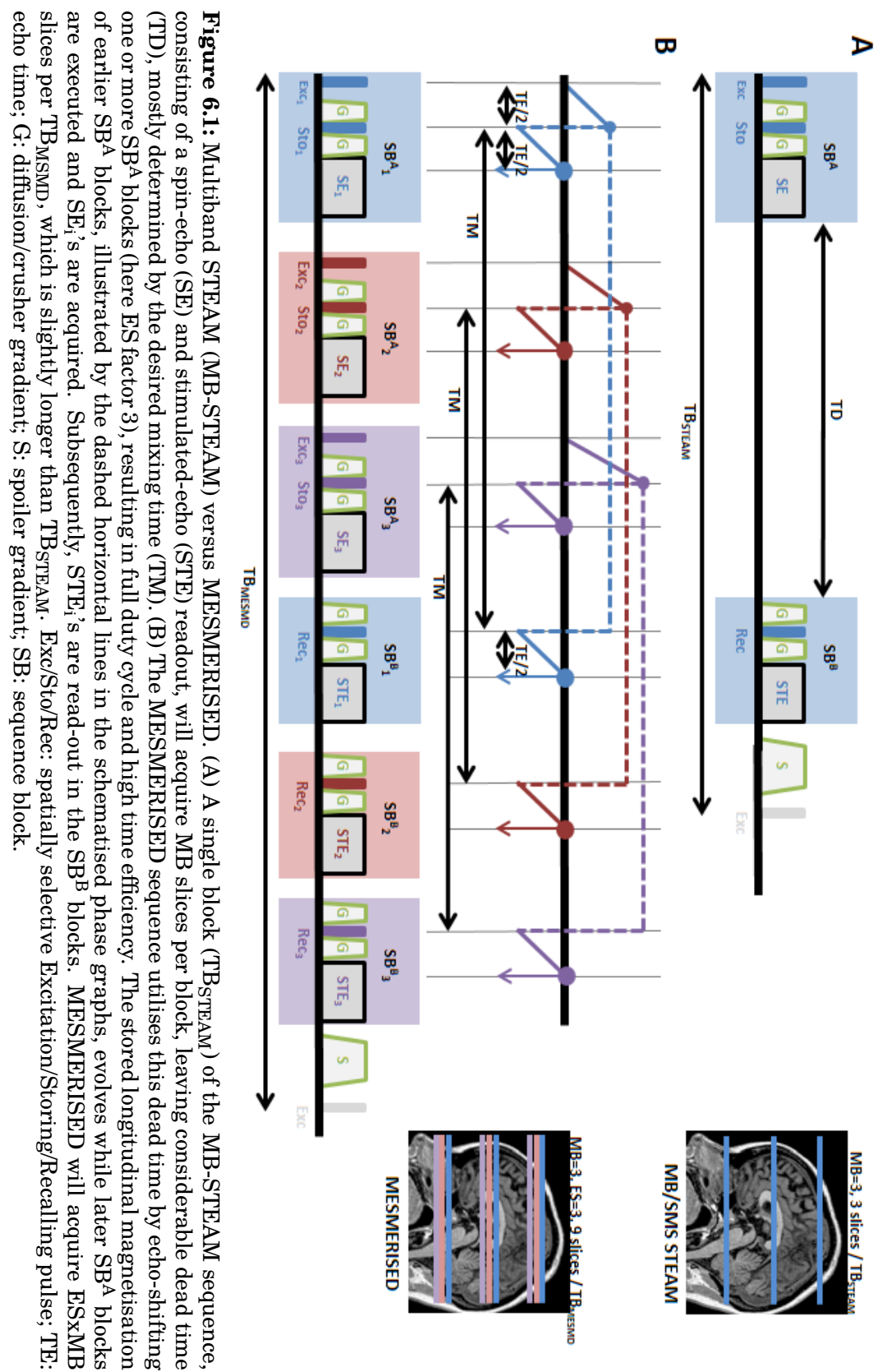




\subsection{Methods}

\subsubsection{MESMERISED sequence implementation}

The MESMERISED sequence was implemented on a Siemens 7 T MAGNETOM platform (Siemens Healthineers Erlangen, Germany). A sequence unit (or building block) was created consisting of a single multiband selective pulse surrounded by two gradient pulses and followed by an echoplanar imaging (EPI) readout. This unit serves both as a $\mathrm{SB}^{\mathrm{B}}$ block and, preceded by a matched multiband pulse, like a $\mathrm{SB}^{\mathrm{A}}$ block (Figure 6.1B). Blipped-CAIPIRINHA simultaneous multi-slice reconstruction [182] with the split-slice GRAPPA leak-block approach [47] was performed by using the online reconstruction that is part of the MGH SMS-EPI release for Siemens platforms (https://www. nmr. mgh . harvard. edu/sof tware/c2p/sms). In-plane acceleration was reconstructed with GRAPPA [94] using FLEET auto-calibration scans as described in reference [28]. The MESMERISED implementation allows for user-controllable MB, GRAPPA and ES acceleration factors, along with the common parameters (TE, TR, number of slices, and resolution parameters: slice thickness, in-plane resolution, readout bandwidth). For diffusion imaging, duration $\delta$, amplitude $|\mathrm{G}|$ and time spacing $\Delta$ of the diffusion gradient pairs are user-controllable to determine b-value and diffusion time for the $\mathrm{SE}\left(\mathrm{b}_{\mathrm{SE}}\right.$ and $\left.\Delta_{\mathrm{SE}}=\Delta\right)$ and the $\mathrm{STE}\left(\mathrm{b}_{\mathrm{STE}}\right.$ and $\left.\Delta_{\mathrm{STE}}=\Delta+\mathrm{TM}\right)$. Diffusion gradient direction tables were implemented as multiples of 12 directions $(24,36,48,60)$ distributed around a full sphere by an electrostatic repulsion algorithm [114], interspersed with $b_{0}$ volumes every 12 directions. Multi-shell tables were implemented by combining tables and suitable modulation of $|\mathrm{G}|$ by scaling the length of the diffusion direction vector. To remove the need for SAR-intensive Fat Saturation (FatSat) pulses, "fat-unfocusing" as described in the work of Ivanov et al. 2010 [107] was achieved by the appropriate setting of the pulse lengths and bandwidth-time product (BWTP).

\subsubsection{MRI data acquisition}

Whole-brain acquisitions were performed on a whole-body research $7 \mathrm{~T}$ Siemens MAGNETOM (Siemens Healthcare, Erlangen, Germany) system with gradients delivering $70 \mathrm{mT} / \mathrm{m}$ maximum strength at a maximum slew rate of $200 \mathrm{~T} / \mathrm{m} / \mathrm{s}$ per physical axis. For RF transmission and reception, a single-channel-transmit/32-channel-receive head coil (Nova Medical, Wilm- 
ington, MA, USA) was used. Written informed consent was obtained from all the subjects before imaging and all procedures were approved by the Ethics Review Committee Psychology and Neuroscience (ERCPN) of the faculty of Psychology and Neuroscience, Maastricht University. MESMERISED was tested on five healthy subjects ( 3 males and 2 females, average weight $75 \pm 5 \mathrm{~kg}$ and age $29 \pm 3$ years). Dielectric pads [200] were used to improve $\mathrm{B}_{1}{ }^{+}$field homogeneity by positioning two pads at the ears/temples. All sessions started with a localizer scan in three orthogonal directions. Subsequently, a $\mathrm{B}_{0}$ fieldmap was acquired [57] and used to optimised $\mathrm{B}_{0}$ shim currents. An absolute $\mathrm{B}_{1}{ }^{+}$map (using a pre-saturation turbo-flash (PreSat-TFL) sequence [51]) was then acquired and used to calibrate the global transmit flip angle.

Subsequently, MESMERISED acquisitions were performed with full brain coverage, with slices in a transverse orientation. EPI readout was executed along the anterior-posterior (AP) direction, and undersampled with GRAPPA acceleration factor 2 or 3 and using a partial Fourier factor of 6/8. In-plane reference (ACS) lines for GRAPPA (24 lines for factor 2, 36 lines for factor 3 ) were acquired using FLEET at a flip angle $12^{\circ}$ with 2 to 5 dummy pre-scans. For blipped CAIPI MB [34, 182], a CAIPI-shift (CS) factor was employed (MB: 3, CS: 2; and MB: 4, CS: 3). Every acquisition was accompanied by 5 volumes with a reversed-phase encoding direction (posterior-anterior) only for the contrast with highest signal (i.e. shortest TM or TE, flip angles $90^{\circ}-90^{\circ}-90^{\circ}$, and/or $b_{0}$ acquisition), used for correction of off-resonance distortions.

MESMERISED protocols were created for imaging of quantitative $\mathrm{T}_{1}\left(\mathrm{q} \mathrm{T}_{1}\right)$, quantitative $\mathrm{T}_{2}\left(\mathrm{qT}_{2}\right)$, transmit field $\left(\mathrm{B}_{1}{ }^{+}\right)$mapping and diffusion. In MESMERISED the number of slices must be an integer multiple of MBxES for all employed MB and ES factors. For $\mathrm{qT}_{2}, \mathrm{~B}_{1}{ }^{+}$and diffusion protocols with a single mixing time or diffusion time $(\Delta)$ this provides a single constraint. For $\mathrm{qT}_{1}$ or diffusion protocols with multiple TM or $\Delta$ 's, this provides a set of constraints, one for each ES/TM combination. These constraints are easily solved by choosing slice numbers $\mathrm{N}$ with a large number of integer divisors (highly composite numbers), such as 60, 72, 90 and 108, which can be used for isotropic imaging resolutions of $2 \mathrm{~mm}, 1.8 \mathrm{~mm}, 1.5 \mathrm{~mm}$, and $1.25 \mathrm{~mm}$, respectively. Each integer divisor of slice number divided by MB factor (i.e. N/MB), then determines an allowable ES factor under the same TR (because it determines a single temporal reordering of sequence blocks, see Supplementary Figure 6.15). For instance, at 72 slices with $\mathrm{MB}=3$, $\mathrm{N} / \mathrm{MB}=24$ the allowable ES factors are [1, 2, 3, 4, 6, 8, 12, 24]. At full duty cycle, each such ES is associated with a single minimum TM, determined 
by the length of RF pulses, gradient pulses, and EPI train length. Again due to MESMERISED's temporal block reordering property, the minimum $\mathrm{TM}$ for each allowable $\mathrm{ES}$ is (very close to) $\mathrm{ES}^{*} \mathrm{TM}_{\mathrm{ES}=1}$, i.e. an integer multiple of the minimum TM for $\mathrm{ES}=1$.

A proof of principle test was performed to demonstrate that MESMERISED's echo-shifting does not affect the resulting SE and STE images, and therefore the same signal can be acquired in shorter total acquisition time. Sets of MESMERISED SE and STE images were acquired at $1.8 \mathrm{~mm}$ isotropic resolution at different $\mathrm{ES}$ but fixed $\mathrm{TM}=300 \mathrm{~ms}$, $\mathrm{TR}=11.88 \mathrm{~s}$ and $\mathrm{TE}$ $=47.5 \mathrm{~ms}$. ESxMB combinations of $1 \times 3,2 \times 3$ and $4 \times 3$ were acquired with transverse slices, GRAPPA 2, Field of view (FoV) $230 \times 230 \mathrm{~mm}^{2}$ and partial Fourier 6/8. The achievable (but unused) minimum TR for ESxMB= $2 \times 3$ and $4 \times 3$ were recorded to quantify the acceleration potential of MESMERISED.

Quantitative $\mathrm{T}_{1}$ imaging was performed at $1.8 \mathrm{~mm}$ isotropic and $1.25 \mathrm{~mm}$ isotropic, by performing relaxometry over a range of allowable ES/TM combinations at the same TR and TE. Five repetitions of each TM were acquired to assess retrospectively what level of data averaging (1, 3 or 5 repetitions) supports robust exponential $\mathrm{T}_{1}$-decay model fitting. Additionally, $\mathrm{T}_{1}$ relaxometry was performed at both at the minimum possible TR, at full duty cycle and acceleration, and with a doubling of TR and all TM's. This is to assess the range of TM's necessary robust exponential $\mathrm{T}_{1}$-decay model fitting. Full acquisition parameters are given in Supplementary Table 6.11 .

Quantitative $\mathrm{T}_{2}$ and $\mathrm{B}_{1}{ }^{+}$mappings were performed at $1.8 \mathrm{~mm}$ by relaxometry on a range of TE's, and by cosine model fitting of Exc-Sto-Rec $=\alpha^{\circ}$ $2 \alpha^{\circ}-\alpha^{\circ}$ for a range of multipliers $\alpha$ [138], respectively. Additionally, $\mathrm{T}_{2}$ relaxometry was performed at both $\mathrm{ES}=1$ and at $\mathrm{ES}=3$, to assess whether robust $\mathrm{qT}_{2}$ mapping can be performed under echo-shifting. Full acquisition parameters are given in Supplementary Table 6.12.

Multi-shell diffusion imaging was performed at $2.0 \mathrm{~mm}$ and $1.8 \mathrm{~mm}$ isotropic for different maximum b-values. Diffusion acquisitions were designed to explore the strengths of MESMERISED in achieving a high number of volumes per unit time, and either high b-values or a large range of $\Delta$ 's. A set multi-shell diffusion acquisitions with maximum b-value $7000 \mathrm{~s} / \mathrm{mm}^{2}, 185$ directions over 4 shells (a total of $370 \mathrm{SE}$ and STE volumes), were performed at $1.8 \mathrm{~mm}$ isotropic and different ES/TM, realising different diffusion times between $80 \mathrm{~ms}$ and $430 \mathrm{~ms}$ in the STE. A 4-shell acquisition at $2 \mathrm{~mm}$ isotropic with maximum b-value $7000 \mathrm{~s} / \mathrm{mm}^{2}$ was performed to explore the effect of a very short TR $(2.36 \mathrm{~s})$ and a very high number of 
volumes per unit time. An acquisition at $1.8 \mathrm{~mm}$ isotropic at four different diffusion times and two different b-values (1000 and $2500 \mathrm{~s} / \mathrm{mm}^{2}$ ) was performed to assess efficient exploration of $\Delta$ dependent effects on Mean Diffusivity (MD) and Mean Kurtosis (MK). Full acquisition parameters are given in Supplementary Table 6.13.

\subsubsection{Image processing and analysis}

Each MESMERISED dataset (all repetitions for all TM's ( $\left(\mathrm{qT}_{1}\right)$, TE's ( $\left.\mathrm{qT}_{2}\right)$, flip angles $\left(\mathrm{B}_{1}{ }^{+}\right)$, or directions and $b$-values for diffusion) was individually denoised, separately for the SE volumes and STE volumes. Denoising was performed before any other step following the approach by Veraart et al. 2016 [215], using the mrdenoise tool in MRtrix 3.0 [205] with a kernel size of 5 or 7 . Subsequently, the data were corrected for movement, off-resonance and eddy-current artefacts with the Topup and Eddy tools using FSL v6.0.1 [110]. Given that the TE, EPI readout-train, and crushing/diffusion gradients are exactly matched between SE and STE within each acquisition, distortion estimation can be performed on either SE or STE. Therefore, Topup was performed on the highest signal SE volumes (i.e. shortest TE, flip angles $90^{\circ}-180^{\circ}-90^{\circ}$, and/or $\mathrm{b}_{0}$ acquisition) and Eddy was performed separately on SE and STE volumes with this Topup input. Since data was organised with shortest-TM volumes first, the first acquired SE and STE volumes are already in close spatial alignment (having been acquired $\sim 100-300 \mathrm{~ms}$ apart in time), which ensures good alignment of all SE and STE volumes. After correction, the following analyses were performed.

$\mathrm{qT}_{1}$ and $\mathrm{qT}_{2}$ analysis

For $\mathrm{qT}_{1}$ analysis, two distinct relaxometry approaches were used which fit volumes at varying TM with the same TR and TE. In the first approach, only the STE volumes are used. The STE signal as a function of TR, TM, $\mathrm{TE}$ and flip angles can be written:

$\left.S_{S T E}\left(T R, T M, T E, \alpha_{i}\right)=0.5 S_{0}\left(\prod_{i=1}^{3} \sin \left(\alpha_{i}\right)\right)\left(\exp \left(\frac{-T M}{T_{1}}\right)-\exp \left(\frac{-T R}{T_{1}}\right)\right)\right) \exp \left(\frac{-T E}{T_{2}}\right)$

Where $\mathrm{S}_{0}$ contains all parameters not varying with TR, TM, TE and flip angles, like proton density and $\mathrm{Tx} / \mathrm{Rx}$ spatial sensitivity profiles, and $\alpha_{1}$, $\alpha_{2}$, and $\alpha_{3}$ are the flip angles (in radians) of excitation, storing, and recall- 
ing pulse, respectively. Absorbing all terms that do not vary with TR or $\mathrm{TM}$ into the $\mathrm{S}_{0}$ term, we get:

$$
S_{S T E}\left(S_{0}, T R, T M, T_{1}\right)=S_{0}\left(\prod_{i=1}^{3} \sin \left(\alpha_{i}\right)\right)\left(\exp \left(\frac{-T M}{T_{1}}\right)-\exp \left(\frac{-T R}{T_{1}}\right)\right)
$$

In the first approach, Equation 6.2 was fitted to STE volumes at varying TM and maps for $S_{0}$ and $T_{1}$ were obtained. In this approach, the $S_{0}$ absorbs effects of flip angle and $\mathrm{T}_{2}$ decay (as well as $\mathrm{RF}$ receive coil sensitivities and proton density), and the fitted curve is a product of exponential functions. In the second approach, both SE and STE volumes are used. The SE signal and STE/SE signal ratio as a function of TR, TM, TE and flip angles can be written:

$$
\begin{gathered}
S_{S E}\left(T R, T M, T E, \alpha_{i}\right)=S_{0} \sin \left(\alpha_{1}\right) \sin \left(\frac{\alpha_{2}}{2}\right)^{2}\left(1-\exp \left(\frac{-(T R-T M)}{T_{1}}\right)\right) \exp \left(\frac{-T E}{T_{2}}\right) \\
\frac{S_{S T E}}{S_{S E}}=\frac{\sin \alpha_{2} \sin \alpha_{3}}{1-\cos \alpha_{2}} \exp \left(\frac{-T M}{T_{1}}\right)
\end{gathered}
$$

Absorbing all terms that do not vary with TR or TM for the ratio into an $\mathrm{S}_{0} *$ term, we get:

$$
\frac{S_{S T E}}{S_{S E}}=S_{0}^{*}\left(\alpha_{i}\right) \exp \left(\frac{-T M}{T_{1}}\right)
$$

In the second approach Equation 6.5 was fitted to STE/SE ratio volumes at varying $\mathrm{TM}$ and maps for $\mathrm{S}_{0} *$ and $\mathrm{T}_{1}$ were obtained. In this approach, the $\mathrm{S}_{0} *$ absorbs effects of flip angle, and the fitted curve is a simple exponential function. Therefore, the second approach uses the available SE images in MESMERISED to establish a potentially lower complexity fit (simple exponential vs. product of exponentials in the first approach). Approach 1 and 2 were both evaluated for the estimation of $\mathrm{qT}_{1}$ maps and compared for the precision of their estimates by evaluating Fisher Information Matrix (FIM) local uncertainty [102]. In addition, the uncertainty of $\mathrm{qT}_{1}$ estimates was evaluated for an increasing number $(1,3,5)$ of repetitions per TM volume. For $\mathrm{qT}_{2}$ mapping only the SE signal was used for both acquisitions $(\mathrm{ES}=$ 1 and 3) separately, fitting a simple exponential decay of TE's to estimate a $\mathrm{qT}_{2}$ map.

$\mathrm{B}_{1}^{+}$analysis

For $\mathrm{B}_{1}{ }^{+}$mapping, the approach used by Lutti et al. 2012 [138] was used on the acquired varying flip angle data. It consisted in taking the ratio between STE and SE signals, where the flip angle ratio between the three 
STEAM pulses is $\alpha^{\circ}-2 \alpha^{\circ}-\alpha^{\circ}$ (e.g. for $\alpha=90^{\circ}$ : $90^{\circ}-180^{\circ}-90^{\circ}$ degrees for excitation, storing and recalling pulses respectively). The STE/SE ratio is proportional to the cosine of the excitation flip angle:

$$
\frac{S_{S T E}}{S_{S E}}=\frac{\sin (2 \alpha) \sin (\alpha)}{\sin (\alpha)^{2}} \exp \left(\frac{-T M}{T_{1}}\right) \rightarrow S_{0}^{*}\left(T M_{0}, b_{0}\right) \cos \alpha
$$

Therefore, the relative $\mathrm{B}_{1}{ }^{+}$map can be estimated from a sufficient number of ratio volumes with varying $\alpha$.

Diffusion analysis

For diffusion MRI, the following analyses were performed. For the 4-shell ( $\max \mathrm{b}=7000 \mathrm{~s} / \mathrm{mm}^{2}$ ) single-TM acquisitions, the diffusion data from the STE signal was analysed by DTI on the 1st shell, Diffusion Tensor Kurtosis [111] on the 1st and 2nd shell, Ball\&Stick models $[26,25]$ with one $\left(B \& S_{r 1}\right)$, two $\left(B \& S_{\mathrm{r} 2}\right)$ and three $\left(B \& S_{\mathrm{r} 3}\right)$ sticks on all shells, NODDI model [237] on the 1st and 2nd shell, CHARMED model [8] with one $\left(\mathrm{CHARMED}_{\mathrm{r} 1}\right)$ and two $\left(\mathrm{CHARMED}_{\mathrm{r} 2}\right)$ cylinder compartments on all shells. For the SE volumes from the same acquisitions, the highest $b$-value shell was analysed with DTI. For the 2-shell multi-TM acquisition, both DTI (1st shell) and DKI (1st and 2nd shell) were used on the STE signal.

Model optimisation

All modelling analyses above were performed in the Microstructure Diffusion Toolbox (MDT; https://mdt-toolbox.readthedocs.io/en/latest_ release/) with Powell optimiser at patience 10, and using a cascaded optimisation approach [101]. For diffusion modelling, the cascade consisted of $\mathrm{S}_{0}$ estimation - B\&S $\mathrm{S}_{\mathrm{r} 1}$ estimation - desired model; for $\mathrm{qT}_{1}, \mathrm{qT}_{2}$ and $\mathrm{B}_{1}{ }^{+}$ analysis: $\mathrm{S}_{0}$ estimation - desired model. Where relevant, the precision of estimates was evaluated by computing standard deviations derived from the FIM [102].

\subsection{Results}

Figure 6.2 shows a comparison between image and signals obtained using MESMERISED with no ES (ESxMB = 1x3, equivalent to MB STEAM, cf. Figure 6.1A), ESxMB $=2 \times 3$ and $4 \times 3$ for the SE and STE images. Qualita- 
tively, Figure 6.1A shows that images retain the same contrast and characteristics when dead time is filled up through echo-shifting. At the same time, the minimum TR (minTR) illustrates that MESMERISED allows these images to be acquired at higher acceleration in a shorter time, going from a $11.88 \mathrm{~s}$ minTR with $\mathrm{ESxMB}=1 \mathrm{x} 3$, to $4.83 \mathrm{~s}$ minTR with $\mathrm{ESxMB}=$ $4 \times 3$. Here we should note that, if the shorter TR is actually used, the contrast and SNR might change compared to the long TR images shown here due to magnetisation and steady-state effects. The minTR also illustrates that although the additional acceleration compared to multiband STEAM is significant (e.g. $2.46 \mathrm{x}$ for $\mathrm{ESxMB}=4 \mathrm{x} 3$ compared to $\mathrm{ESxMB}=1 \mathrm{x} 3$ ), and multiplicative with $\mathrm{MB}$ factor (e.g. 7.38x for $\mathrm{ESxMB}=4 \mathrm{x} 3$ compared to $\mathrm{ESxMB}=1 \mathrm{x} 1$ ), the temporal acceleration factor is generally not linear with ES, due to the time added by the $\mathrm{SB}^{\mathrm{B}}$ (i $>$ 1) blocks (c.f. Figure 6.1B). Figure 6.1B shows a more direct comparison in signal line plots. MESMERISED accelerated signals $(2 \times 3$ and $4 \times 3)$ follow the MB STEAM signal (1x3) closely.

\subsection{1 $\mathrm{qT}_{1}$ mapping}

Figure 6.3A shows MESMERISED $\mathrm{T}_{1}$ relaxometry data at $1.8 \mathrm{~mm}$ isotropic. The relaxometry on TM is performed at constant TE $(28 \mathrm{~ms})$ and TR $(6.9 \mathrm{~s})$ by increasing the ES factor proportionally to TM, for TM's from $140 \mathrm{~ms}$ to $1680 \mathrm{~ms}$ (see Supplementary Table 6.11). This results in total acceleration factors (ESxMB) from 3 to 36, highlighting the super-acceleration capability of MESMERISED for $\mathrm{T}_{1}$ relaxometry. For MB-STEAM and STEAM (without MB) the TR for relaxometry would be limited by the largest TM and would equal 41.51 and $124.60 \mathrm{~s}$, respectively, which means that relative temporal speed-ups of $6.02 \mathrm{x}$ and $18.06 \mathrm{x}$ are achieved compared to MB-STEAM and STEAM, respectively. Figure 6.3B shows the whole-brain isotropic resolution $\mathrm{S}_{0}$ and $\mathrm{qT}_{1}$ maps resulting from analysis of STE data (i.e. using Equation 6.2). The $\mathrm{S}_{0}$ map estimated in this way retains strong $\mathrm{T}_{2}$-weighting (along with weighting for proton density and $\mathrm{Tx} / \mathrm{Rx}$ fields), which is recognised in contrast between white matter (WM) and grey matter (GM) structures. The $\mathrm{qT}_{1}$ map shows also a high contrast level due to the quantitative differences in $\mathrm{T}_{1}$ between $\mathrm{WM}$ and GM structures. For comparison, Figure 6.4A shows $\mathrm{S}_{0}{ }^{*}$ and $\mathrm{qT}_{1}$ maps resulting from analysis of the STE/SE data from the same acquisition (i.e. using Equation 6.5). The resulting $\mathrm{qT}_{1}$ map is qualitatively highly similar. However, the theoretical expectation (described above) that the $\mathrm{S}_{0} *$ map is more weighted 


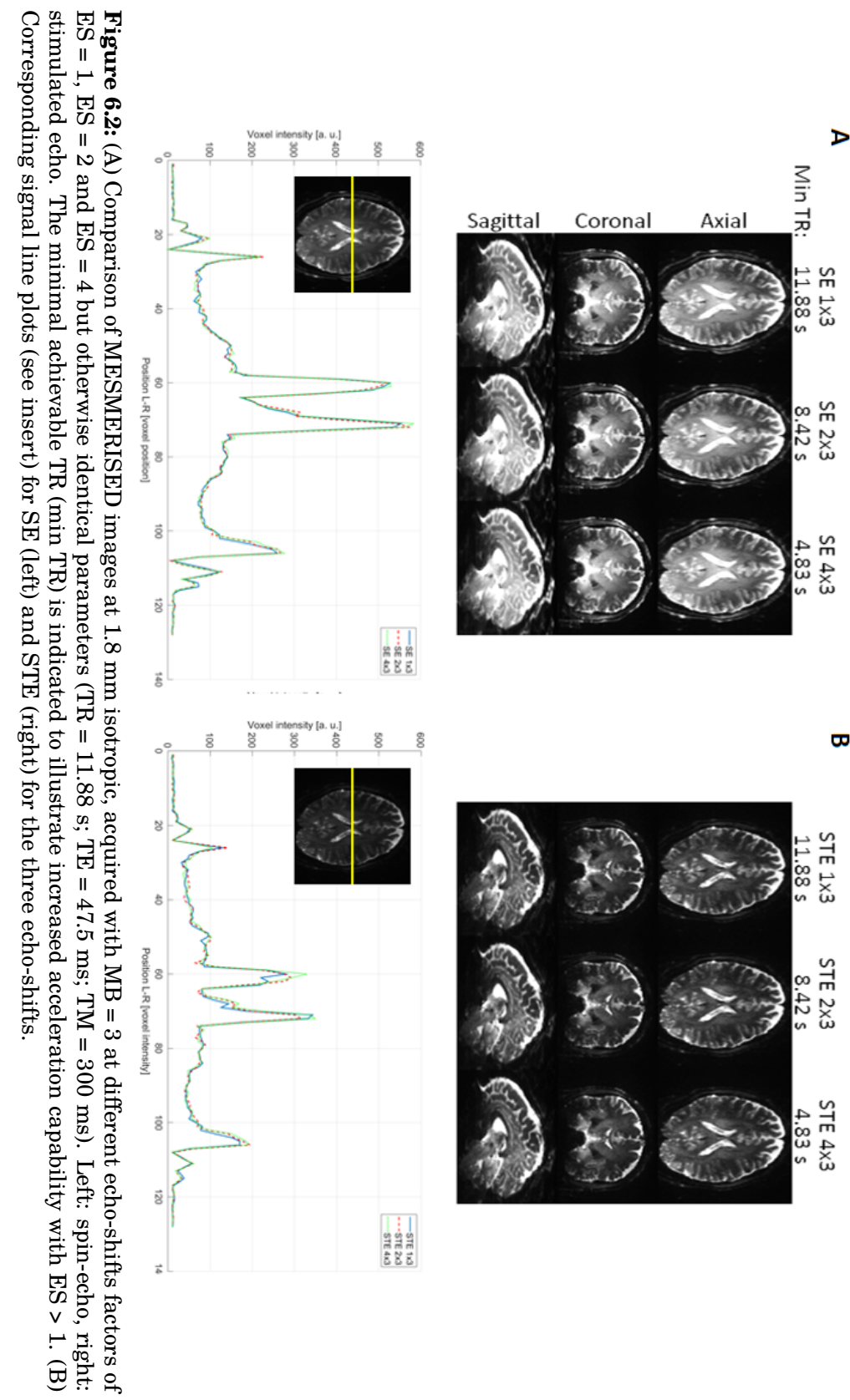


for Tx field (i.e. local flip angle) and less for $\mathrm{T}_{2}$ compared to the $\mathrm{S}_{0}$ map is borne out in a decreased WM/GM contrast and an increased central darkening strongly (anti-)correlated with the transmit field (described below). Figure 6.4B shows MESMERISED $\mathrm{S}_{0}{ }^{*}$ and $\mathrm{qT}_{1}$ maps at $1.25 \mathrm{~mm}$ isotropic, from data at constant TE $(31.7 \mathrm{~ms})$ and TR $(8.8 \mathrm{~s})$, resulting in total acceleration factors (ESxMB) from 3 to 54 over 8 different TM's (Supplementary Table 6.11). For MB-STEAM and STEAM (without MB) the TR for relaxometry would be limited by the largest TM and would equal 79.79 and $239.40 \mathrm{~s}$, respectively, which means that relative temporal speed-ups of 9.07x and 27.20x are achieved compared to MB-STEAM and STEAM, respectively. A more detailed comparison of the uncertainty of $\mathrm{qT}_{1}$ maps for the more SNR challenged $1.25 \mathrm{~mm}$ isotropic data is shown in Supplementary Figure 6.16. This shows that uncertainty in $\mathrm{qT}_{1}$ estimates is lower 1 ) with an increasing number of repetitions, 2$)$ at long TR $(8.8 \mathrm{~s})$ and maximum TM (max TM, 2.16s) compared to short TR (4.4 s) and max TM (1.08 s), and 3) with STE/SE ratio analysis compared to STE-only analysis. Although 1) and 2) would be expected given the averaging effect of repetitions and a max TM that is better for the range of expected $\mathrm{T}_{1}$ 's at $7 \mathrm{~T}(1-1.5 \mathrm{~s})$, the benefit of STE/SE ratio analysis is more interesting. With the STE/SE ratio analysis the local maxima in uncertainty for the long TR, TM data is at a similar level with a single repetition (acquisition time 1:40) as for the STE-only analysis with 5 repetitions (acquisition time 6:22); the STE/SE ratio analysis with three repetitions (acquisition time 4:01) has lower uncertainty everywhere (Supplementary Figure 6.16, bottom row). Additionally, the uncertainty for STE/SE ratio analysis is more homogeneous over the brain. Therefore, the $1.25 \mathrm{~mm}$ isotropic whole-brain $\mathrm{qT}_{1}$ map in Figure 6.4 can be achieved in 2-4 minutes, and the $1.8 \mathrm{~mm}$ isotropic $\mathrm{qT}_{1}$ map in 1-2 minutes, depending on the desired precision level.

\subsection{2 $\mathrm{qT}_{2}$ and $\mathrm{B}_{1}$ mapping}

Figure 6.5 shows $\mathrm{T}_{2}$ relaxometry results and $\mathrm{q}_{2}$ maps at $1.8 \mathrm{~mm}$ isotropic with MESMERISED both at ES $=3$ and ES $=1$ (i.e. MB-STEAM). Figure 6.5A shows $\mathrm{SE}$ volumes over echo times of $28.3 \mathrm{~ms}$ to $88.3 \mathrm{~ms}$ (see Supplementary Table 6.12) illustrating $\mathrm{T}_{2}$ decay, qualitatively similar for $\mathrm{ES}=1$ and $\mathrm{ES}=3$. Figure $6.5 \mathrm{~B}$ shows the derived $\mathrm{qT}_{2}$ maps for each $\mathrm{ES}$ factor. Analogous to Figure 6.2, neither the SE signal nor the $\mathrm{qT}_{2}$ map seem affected by the echo-shifting mechanism in MESMERISED. Figure 
6 MESMERISED: Super-accelerated 7 T STEAM imaging for quantitative multi-contrast and diffusion MRI

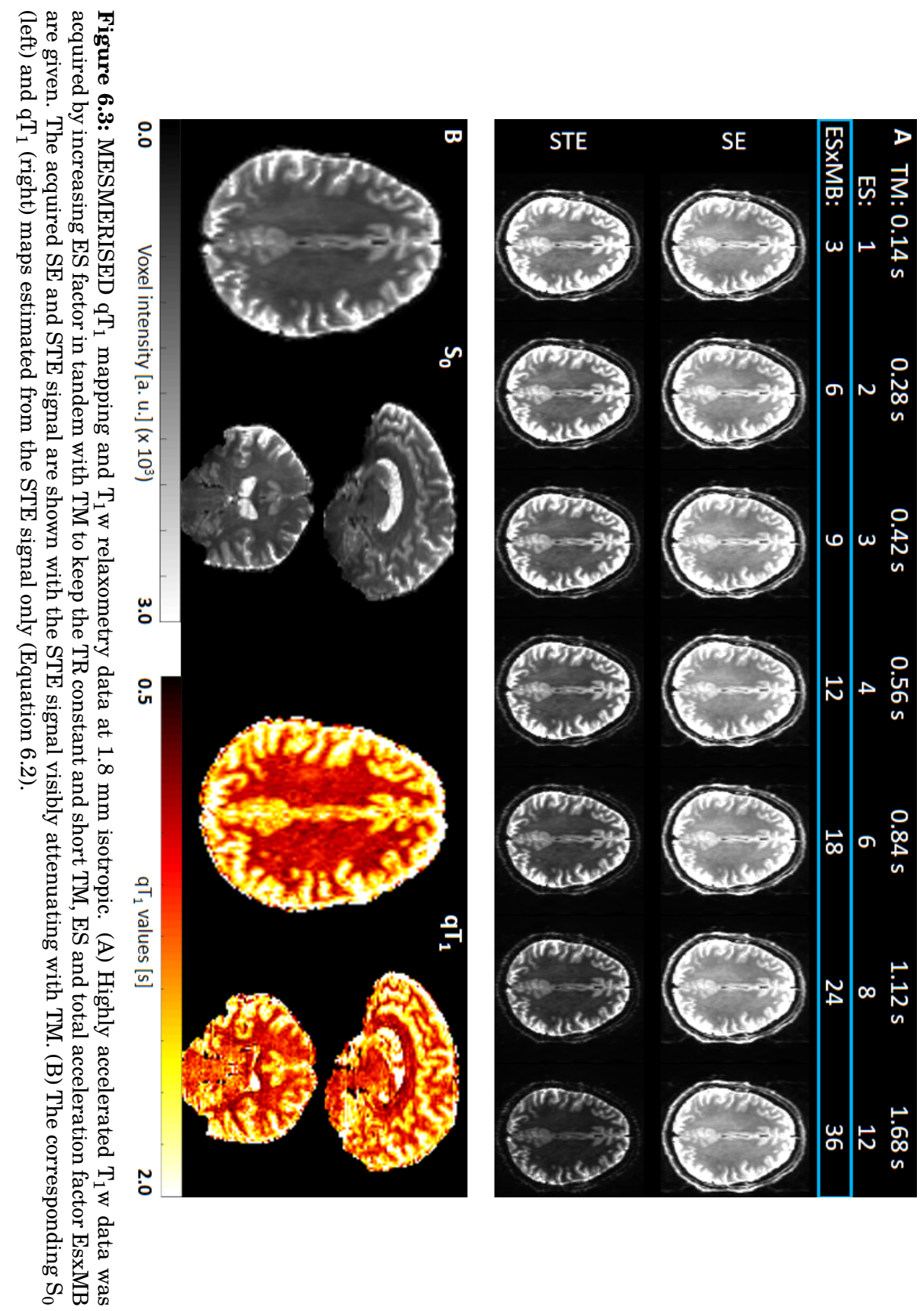




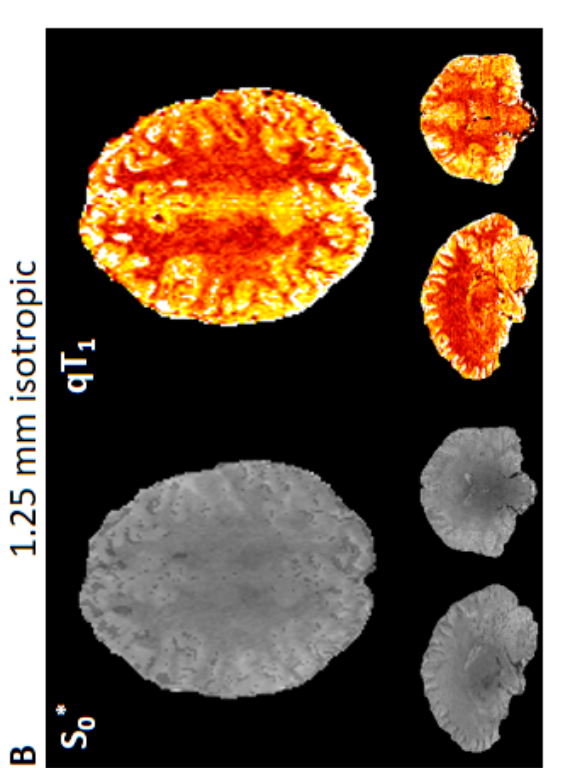

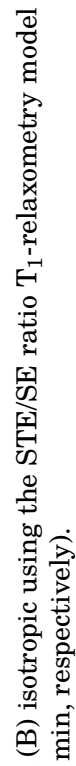

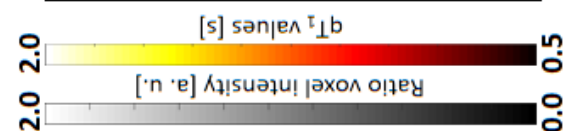

घี ํㅜㅁ ำ ํㅠำ

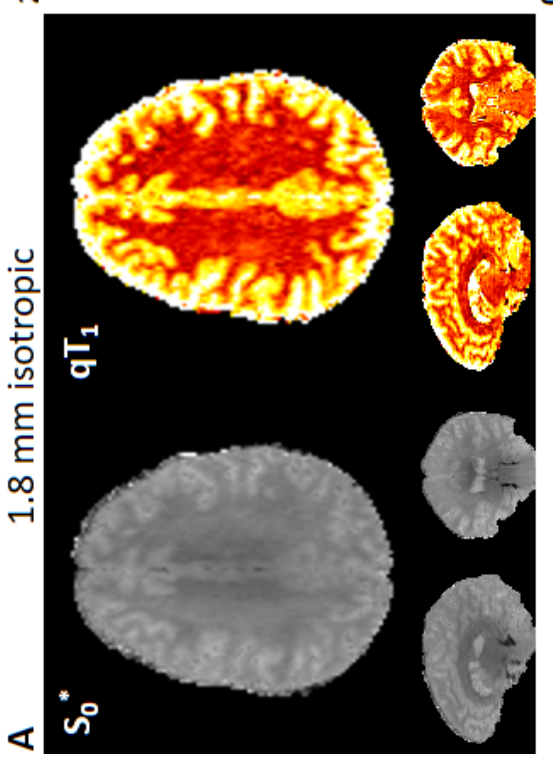

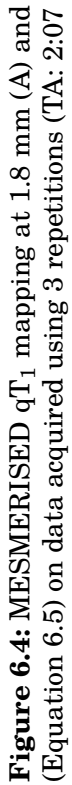


6 MESMERISED: Super-accelerated 7 T STEAM imaging for quantitative multi-contrast and diffusion MRI

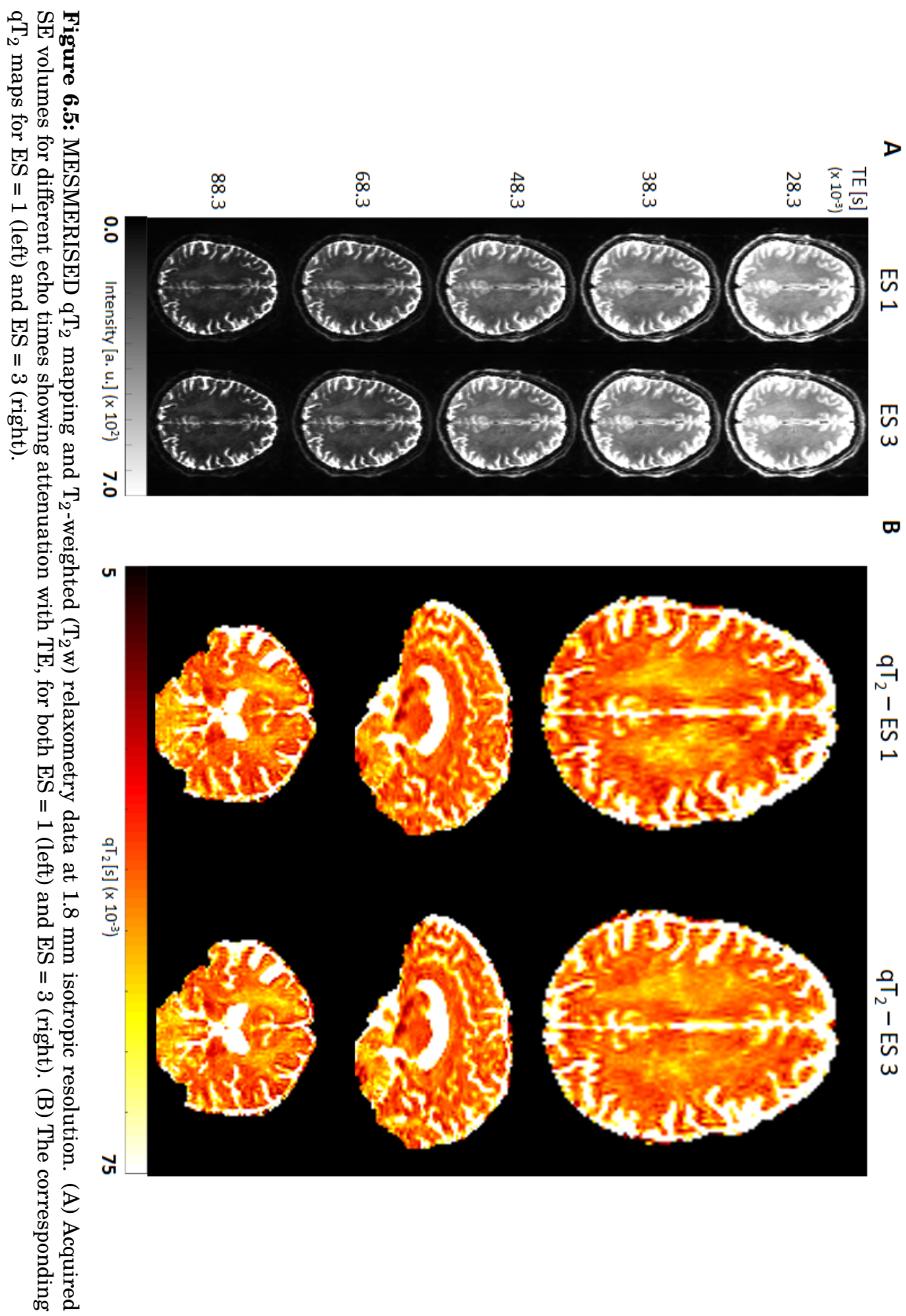


6.6 shows MESMERISED $\mathrm{B}_{1}{ }^{+}$mapping at $1.8 \mathrm{~mm}$ isotropic, derived from both the SE and STE volumes (Figure 6.6A) for different $\alpha_{\mathrm{TX}}$ multipliers in an $\alpha_{\mathrm{TX}}-2 \alpha_{\mathrm{TX}}-\alpha_{\mathrm{TX}}$ flip angle scheme for Exc - Sto - Rec pulses. Figure 6.6B shows the corresponding SE/STE ratios used for $\mathrm{B}_{1}{ }^{+}$map fitting, and Figure 6.6C depicts the resulting whole-brain relative $\mathrm{B}_{1}{ }^{+}$map. Both the results in Figure 6.5B and 6.6C were derived from 3 repetitions per volume, which means that $\mathrm{qT}_{2}$ and $\mathrm{B}_{1}{ }^{+}$mapping can each be achieved in roughly 2 minutes, independently of echo-shift factor used. Here it is important to note that $\mathrm{qT}_{2}$ and $\mathrm{B}_{1}{ }^{+}$mapping, per se, do not benefit from the acceleration by echo-shifting in MESMERISED. However, the ability to perform fast $\mathrm{qT}_{2}$ and $\mathrm{B}_{1}{ }^{+}$mapping with the same pulse sequence that provides accelerated $\mathrm{qT}_{1}$ and diffusion imaging can have considerable advantages for fast multi-contrast mapping.

\subsubsection{Diffusion MRI}

Figure 6.7 illustrates the two-dimensional parameter space of diffusion time (vertical) and b-value (horizontal) with MESMERISED diffusion data at $1.8 \mathrm{~mm}$ isotropic (where the further 2 dimensions of diffusion direction on the sphere are left implicit). Here, different diffusion times were realised by varying TM and utilising the maximum echo-shift factor allowed at each TM, individually minimising TE and TR. All optimised TR's are close to $3.4 \mathrm{~s}$ (see Supplementary Table 6.13), showing a capacity to explore high b-values, up to $7000 \mathrm{~s} / \mathrm{mm}^{2}$, and high diffusion times, up to $436.9 \mathrm{~s}$, at very high acceleration and data rate. In the following, we first focus on the possibilities afforded by multi-shell acquisitions at a single diffusion time and then demonstrate the possibilities of acquisitions at varying diffusion time.

\subsubsection{Multi-shell diffusion MRI}

Figure 6.8 shows example slices out of a 4-shell MESMERISED acquisition and analysis results for the simultaneously acquired STE images (at a diffusion time of $160.6 \mathrm{~ms}$ and b-values up to $7000 \mathrm{~s} / \mathrm{mm}^{2}$ ) and SE images (at a diffusion time of $20.6 \mathrm{~ms}$ and b-values up to $752 \mathrm{~s} / \mathrm{mm}^{2}$ ). This makes explicit how the acquisition of both diffusion-weighted SE volumes and STE volumes provides 8 useful shells of diffusion data in a 4-shell acquisition, even when analysed separately. Whereas the low-b SE volumes support analysis with Gaussian diffusion models such as the DTI model, 


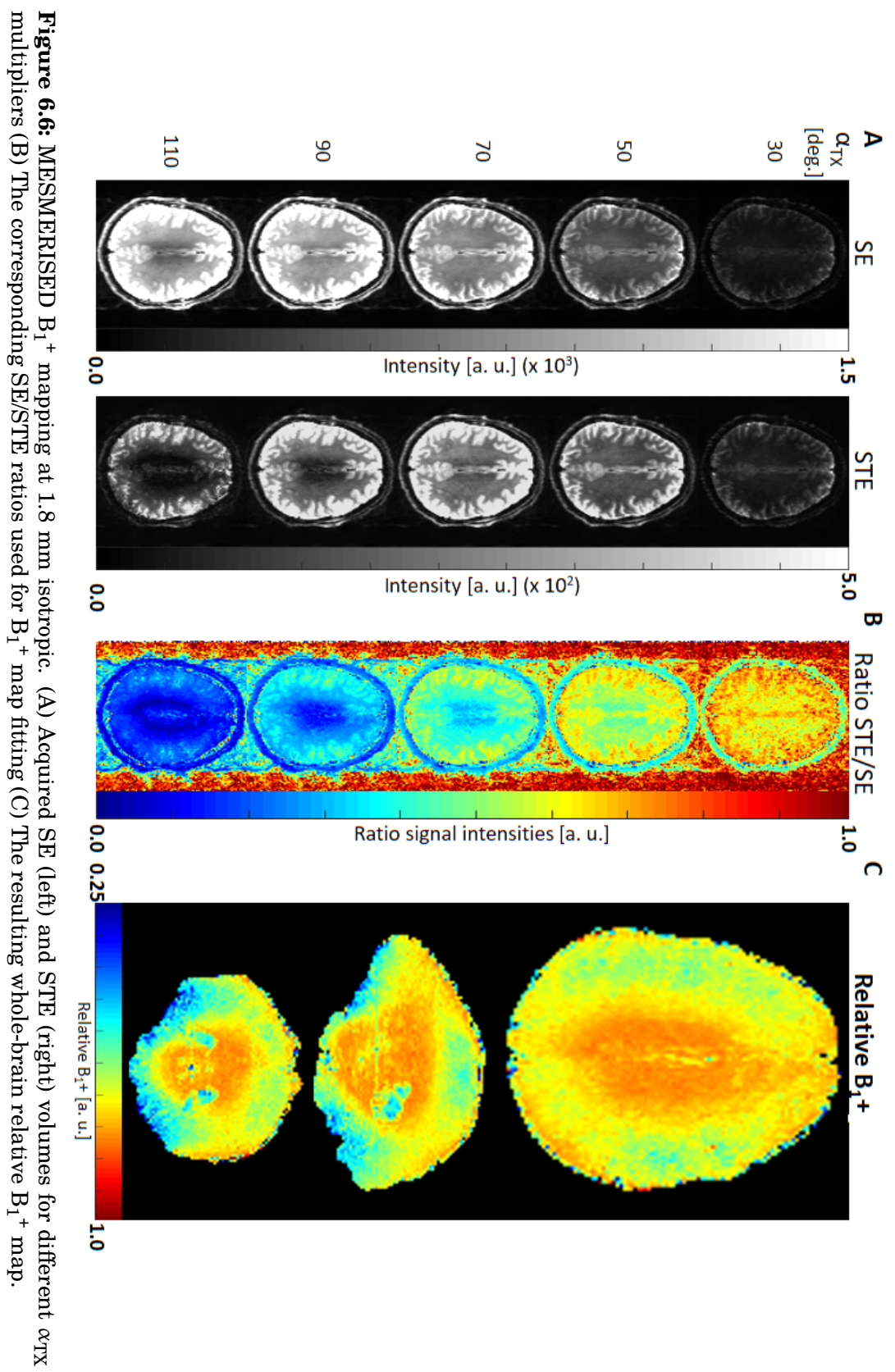


$\frac{1}{E}$
है
$\frac{0}{0}$
$\frac{0}{3}$
$\frac{\pi}{1}$

옷

을

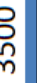

$\therefore$ (3)

$\therefore$ (3)

$4 \rightarrow-5 \mid \frac{5}{4}$

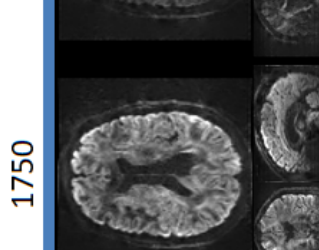

su 9.09L
急望

ㅍ

तี

is :

उ

용

ฮ్

울

$>$ 유

$\checkmark \mathrm{s}$

\&

동

ฐี ฮ્ّ

कै कै

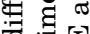

돈

$\circ$. 잉

迄兔

过产产

कo

원

तี

की

금

훙 혐

둥

.7 है क

घี 듬

a 0

-

त

ฐ

त्ञ

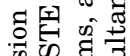

프므

㱐这的

훗

四

월 贾

की

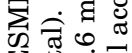

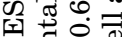

运

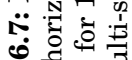
نำ 워

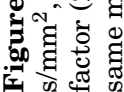

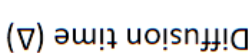


the high-b STE volumes are suitable for non-Gaussian diffusion models, such as the DKI, NODDI and CHARMED models. This data was acquired at $\mathrm{ES}=2$ at a TR of $3.45 \mathrm{~s}$ and a TE of $49.8 \mathrm{~ms}$, which tunes the diffusion time of the STE volumes to the shorter end of the achievable range and the b-values of the SE volumes to the higher end, which is accompanied with a higher TE. When a longer diffusion time and higher echo-shift factor is chosen for the same STE volume maximum b-value (e.g. $7000 \mathrm{~s} / \mathrm{mm}^{2}$ here), diffusion gradient length $\delta$ naturally decreases, along with the SE maximum b-value (see Supplementary Table 6.14).

Figure 6.9A shows two 4 -shell $\left(\max -\mathrm{b}=7000 \mathrm{~s} / \mathrm{mm}^{2}\right)$ acquisitions which realise full duty cycle at similar mixing and diffusion time at $2 \mathrm{~mm}$ isotropic $(\mathrm{ES}=5, \mathrm{TM}=290 \mathrm{~ms}$; top $)$ and $1.8 \mathrm{~mm}(\mathrm{ES}=4, \mathrm{TM}=280 \mathrm{~ms}$; bottom $)$. The $2 \mathrm{~mm}$ acquisition achieves this at a shorter TR $(2.36 \mathrm{~s}$ vs. $3.37 \mathrm{~s})$ and therefore enables a higher data rate of $\sim 1.2 \mathrm{~s}$ per volume (SE and STE together), which generates the full 370 volume dataset in an acquisition time of $7 \mathrm{~min}$ and $47 \mathrm{~s}$ (vs. $10 \mathrm{~min}$ and $53 \mathrm{~s}$ for $1.8 \mathrm{~mm}$ ). Figure 6.9B shows analysis results for two 4-shell acquisitions with matched full-duty cycle TR (3.45 s) and acquisition time (11:08) at $1.8 \mathrm{~mm}$ with different echo-shift factors (2 vs. 3 ) and mixing times ( $140 \mathrm{~ms}$ vs. $210 \mathrm{~ms})$. Besides a different diffusion time for the STE volumes, this also achieves a different maximum $\mathrm{b}$-value for the SE volumes (see Supplementary Table 6.14). As shown below, the capability to realise different diffusion times for the STE volumes at the same TR opens the possibility to explore diffusion times (when the $\mathrm{TE}$ and $\delta$ are kept constant at the same b-value) rather than b-values (at the same diffusion time).

\subsubsection{Varying diffusion time}

Analogous to keeping TR constant in MESMERISED $\mathrm{T}_{1}$ relaxometry (by increasing ES factor proportionally to TM), different diffusion times for the STE volumes at the same TR can be realised by increasing ES factor with increasing diffusion time. When TE, b-value and diffusion gradient length are also kept constant, this opens the possibility to explore the behaviour of diffusion phenomena over different diffusion times. Figure 6.10 illustrates this with 2 -shell diffusion data acquired at four diffusion times with otherwise matched parameters (i.e. TR, TE, $\delta$ and b-value are equal throughout, see Supplementary Table 6.13). Each $1.8 \mathrm{~mm}$ isotropic dataset had $24 \mathrm{di}$ rections at $b=1000 \mathrm{~s} / \mathrm{mm}^{2}, 48$ directions at $b=2500 \mathrm{~s} / \mathrm{mm}^{2}$ and $8 b_{0}$ 's, for a total of 70 STE volumes (and 70 SE volumes, not shown). This is sufficient 


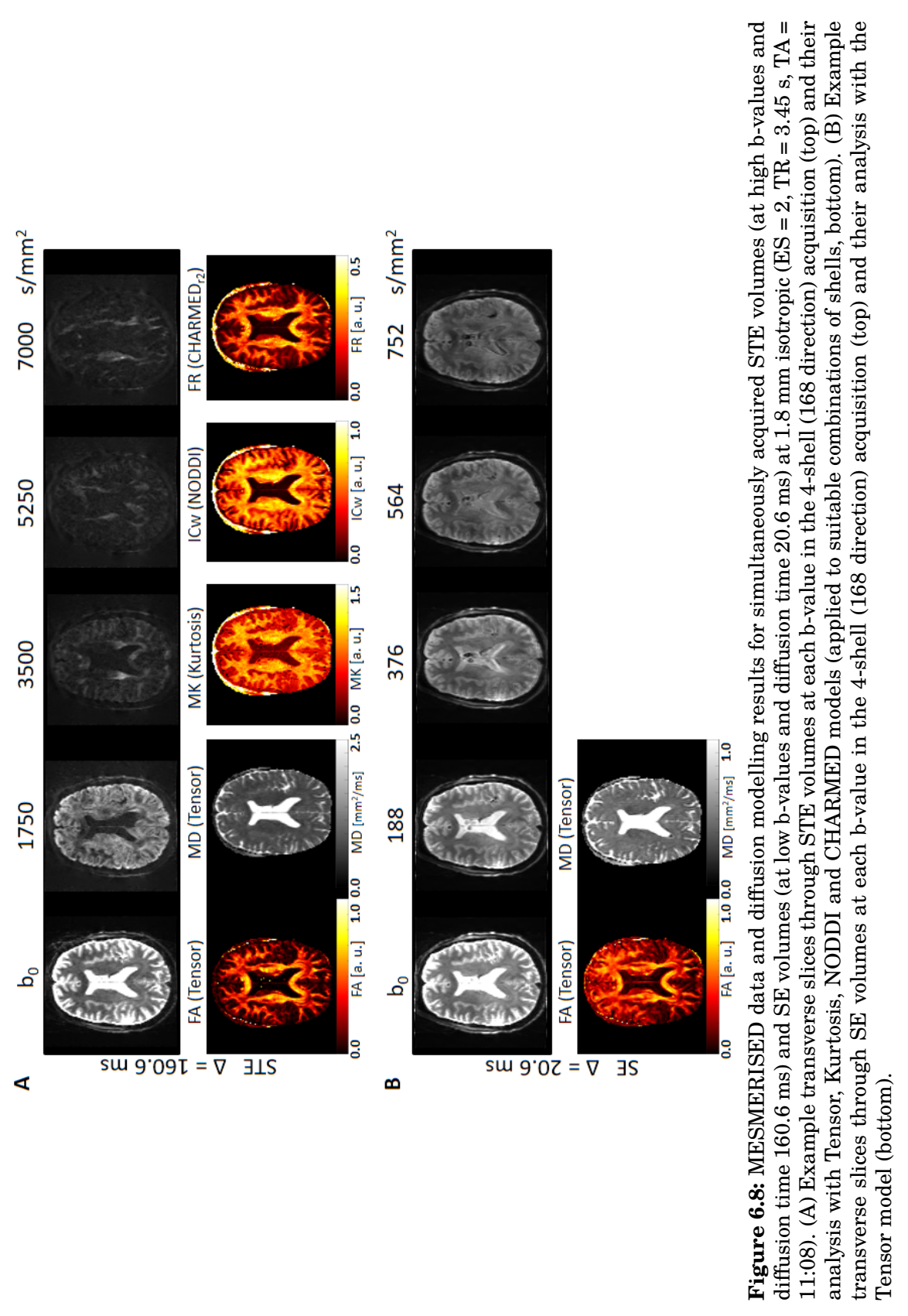




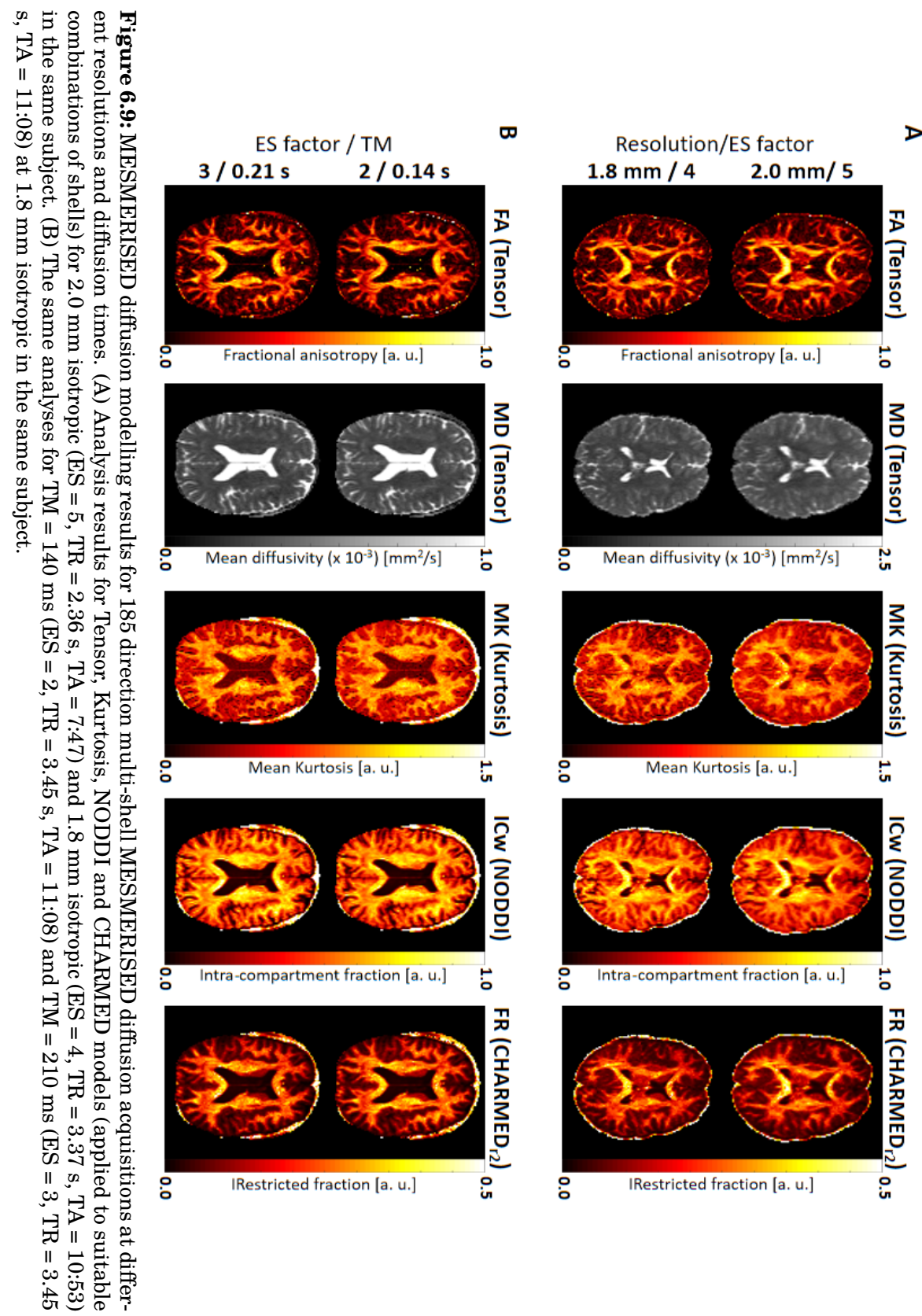


for whole-brain DTI analysis (on the $b=1000 \mathrm{~s} / \mathrm{mm}^{2}$ shell, Figure $6.10 \mathrm{~B}$ ) and DKI analysis (on both shells) at each diffusion time (Figure 6.10C), and to assess how diffusion measures such as Mean Diffusivity (MD) and Mean Kurtosis (MK) change with $\Delta$ (Figure 6.10D).

\subsection{Discussion}

MESMERISED achieves super-accelerated 7 T STEAM imaging by combining echo-shifting and multiband/simultaneous multi-slice acceleration, leading to very high multiplicative acceleration factors and time efficiency for $\mathrm{qT}_{1}$ and diffusion imaging. Furthermore, $\mathrm{qT}_{2}$ and $\mathrm{B}_{1}{ }^{+}$mapping can be performed with the same pulse sequence. MESMERISED inherits from STEAM the capacity to simultaneous generate both SE images and STE images and the possibility of performing $\mathrm{qT}_{1}, \mathrm{qT}_{2}, \mathrm{~B}_{1}{ }^{+}$mapping as well as diffusion-weighted imaging. It also inherits the property of $50 \%$ lower SNR and 40\% lower SAR compared to a similar spin-echo sequence, and 66\% lower SAR compared to an inversion-prepared spin-echo sequence. In essence, MESMERISED capitalises on the low SAR of the STEAM sequence to considerably increase its SNR per unit time through the multiplicative ESxMB acceleration. This enables fast multi-contrast mapping over the whole-brain, for instance at $1.8 \mathrm{~mm}$ isotropic, with $\mathrm{qT}_{1}$ mapping (2:07), $\mathrm{qT}_{2}$ mapping (1:48), $\mathrm{B}_{1}{ }^{+}$mapping (2:14) and 370 volume multi-shell diffusion (11:08), in a little over 17 minutes. Accepting a lower precision level in the quantitative maps (i.e. working with a single, rather than three repetitions), and 200 volumes in the diffusion dataset (cf. Supplementary Tables 6.11, 6.12 and 6.13), would lower the total multi-contrast acquisition time to under 10 minutes (i.e. $\mathrm{qT}_{1}$ mapping in $1: 18, \mathrm{qT}_{2}$ mapping $0: 56$, $\mathrm{B}_{1}{ }^{+}$mapping 1:05 and 200 volume multi-shell diffusion in 6:15).

\subsection{1 $\mathrm{qT}_{1}$ mapping}

MESMERISED $\mathrm{T}_{1}$ relaxometry can be performed at a low constant $\mathrm{TR}$ by increasing the ES factor proportionally to TM, which results in increasing total acceleration factors (e.g. from 3 to 36 in $1.8 \mathrm{~mm}$ data and from 3 to 54 in $1.25 \mathrm{~mm}$ data in Figure 6.4), highlighting the super-acceleration capability of MESMERISED for $\mathrm{qT}_{1}$ imaging. Although the temporal speed-up of the multiplicative ESxMB acceleration is not linear with ES, the speedup compared to multiband only accelerated STEAM can be very large (e.g. $6.02 \mathrm{x}$ faster than $\mathrm{MB}=3$ for $1.8 \mathrm{~mm}$ and $9.07 \mathrm{x}$ faster than $\mathrm{MB}=3$ for 


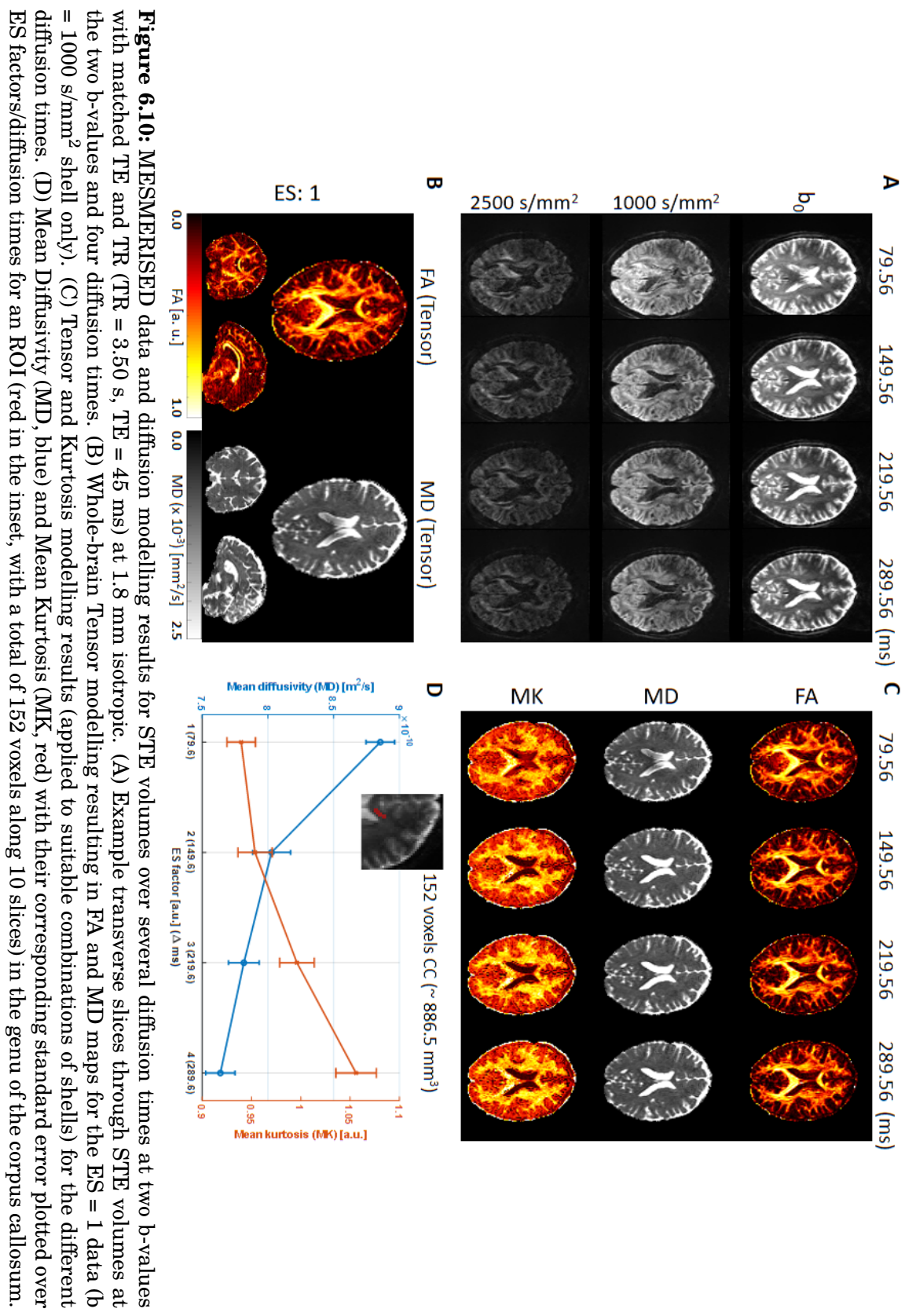


$1.25 \mathrm{~mm}$ ). In addition, MESMERISED simultaneously provides spin-echo images in the same acquisition time, which can be used to either improve the precision of the $\mathrm{qT}_{1}$ maps in the same acquisition time or achieve even shorter acquisition times for the same precision level. For instance, uncertainty in $1.25 \mathrm{~mm}$ isotropic $\mathrm{qT}_{1}$ maps at a single repetition (acquisition time 1:40) with STE/SE ratio analysis are at similar levels as that of three repetitions (acquisition time 4:01) or even 5 repetitions (acquisition time 6:22) for STE-only analysis (Supplementary Figure 6.16, bottom row). Here it should be noted that these results are obtained at less than $100 \%$ duty cycle, with a doubling of the minimum achievable TR and TM (c.f. Supplementary Table 6.11, row 2 and 3), providing a better spacing and range of TM's for $T_{1}$ relaxometry. Potentially, in the future, this space can be used for simultaneous sampling of other contrasts, such as a multi-echo EPI readout [166] for $\mathrm{qT}_{2} / \mathrm{qT}_{2}{ }^{*}$ mapping.

\subsection{2 $\mathrm{qT}_{2}$ and $\mathrm{B}_{1}{ }^{+}$mapping}

Both $\mathrm{qT}_{2}$ and $\mathrm{B}_{1}{ }^{+}$mapping can be performed over the whole-brain with MESMERISED (e.g. $1.8 \mathrm{~mm}$ isotropic $\mathrm{qT}_{2}$ and $\mathrm{B}_{1}{ }^{+}$maps in roughly 2 minutes each; Figure 6.5B and 6.6C). Here we note that $\mathrm{qT}_{2}$ and $\mathrm{B}_{1}{ }^{+}$mapping, per se, do not benefit from the accelerating echo-shifting capacity in MESMERISED. $\mathrm{qT}_{2}$ and $\mathrm{B}_{1}{ }^{+}$mapping derive from basic properties of the STEAM sequence, i.e. relaxometry on SE or STE echo times and the $\alpha$ $2 \alpha-\alpha$ technique, respectively, which can be performed independently of any echo-shifting. However, the ability to perform $\mathrm{qT}_{2}$ and $\mathrm{B}_{1}{ }^{+}$mapping with the same pulse sequence that provides highly accelerated $\mathrm{qT}_{1}$ and diffusion imaging has considerable advantages for fast multi-contrast mapping. First, all quantitative contrasts are generated by the same sequence and readout train leading to similar image distortions which aid alignment of the different maps where needed. Second, for the same reason, the images from all contrasts share the same image noise distribution, which aids denoising with methods that take all images into account [54, 215]. Third, well-aligned $\mathrm{B}_{1}{ }^{+}$maps can help in correcting transmit field related biases in quantitative maps, for instance in $\mathrm{qT}_{1}$ maps [142].

\subsubsection{Diffusion MRI}

For diffusion imaging MESMERISED inherits from STEAM the capacity to achieve long diffusion times and high b-values, as well as the possibility 
to simultaneously generate diffusion-weighted SE images and diffusionweighted STE images. MESMERISED includes to this a very high data rate to explore a large range of b-values, diffusion directions or diffusion times in a short time. For instance, 370 whole-brain diffusion volumes over eight $b$-value shells up to $b=7000 \mathrm{~s} / \mathrm{mm}^{2}$ can be generated at $2 \mathrm{~mm}$ isotropic in $7 \mathrm{~min}$ and $47 \mathrm{~s}(\mathrm{ESxMB}=5 \mathrm{x} 3)$, a data rate of almost a volume per second, or at $1.8 \mathrm{~mm}$ isotropic in $10 \mathrm{~min}$ and $53 \mathrm{~s}(\mathrm{ESxMB}=4 \mathrm{x} 3$; Figure $6.9 \mathrm{~A}$ ), a data rate of more than a volume per 2 seconds. In addition, this is achieved with a lower diffusion gradient duty cycle (and less gradient coil heating and accompanying cooling requirements) than PGSE, since the longer diffusion times in STEAM dictate shorter diffusion gradient duration for the same maximum b-value. The shells consist of half SE volumes in low-b shells which can support analysis with Gaussian diffusion models as in DTI, and half STE volumes in the same number high-b shells which are suitable for non-Gaussian diffusion models, such as DKI, NODDI and CHARMED models. This provides a wealth of diffusion information from several different models in short acquisition time, which could be further extended in future work. For instance, instead of collecting a relatively large number of directions in 4 shells for each SE and STE, fewer (e.g. 6-12) directions could be acquired at each of many (e.g. 14-28) b-values. When carefully chosen, this would continue to support fitting of the aforementioned models on high-b STE volumes, while creating an opportunity to additionally fit the intra-voxel incoherent motion (IVIM) model [126] to the richly sampled low b-value domain in the SE volumes. Further tuning potential for the b-values of SE volumes (for a given STE max-b) lies in the choice of mixing time (and therefore $\Delta$ ) of the STE volumes because, for the same TR and STE $b$-value, the SE $b$-value varies with different choices of TM and $\delta$ (see Supplementary Table 6.14).

The combination of high data rate and wide range of achievable diffusion times makes MESMERISED an excellent tool to investigate the behaviour of diffusion phenomena at long and varying diffusion times. Several studies have shown that processes such as coarse-graining or exchange leave their mark in the change of diffusion MRI measures with increasing diffusion time [39, 154, 157]. Diffusion time-resolved dMRI can even be used to achieve some sensitivity to axonal diameter distributions [5, 12,65]. Most of these applications require a quite extensive sampling of diffusion times, b-values and, sometimes, directions, often leading to long (e.g. 30 $60 \mathrm{~min}$ ) acquisitions with current techniques. MESMERISED can achieve time-efficient sampling of different diffusion times in the STE volumes at otherwise matched parameters by increasing ES factor with increasing dif- 
fusion time. We demonstrate this with $1.8 \mathrm{~mm}$ isotropic diffusion data acquired at four diffusion times from $\sim 80 \mathrm{~ms}$ to $\sim 290 \mathrm{~ms}$, which supports both DTI and DKI at each diffusion time (Figure 6.10). The analysis of the resulting STE data with DTI and DKI captures a dependence of MD and MK with increasing diffusion time in WM (Figure 6.10D), in agreement with earlier reports [83, 112]. In the future, MESMERISED could be a useful tool in further investigating such micro- and meso-structural phenomena. In principle, less than the number of directions acquired here per $\mathrm{b}$-value ( 24 for $\mathrm{b}=1000 \mathrm{~s} / \mathrm{mm}^{2}, 48$ for $\mathrm{b}=2500 \mathrm{~s} / \mathrm{mm}^{2}$ at a total acquisition time of $\sim 17$ min for all diffusion times) might be sufficient for DKI and thus a shorter MESMERISED acquisition may be possible for this purpose. Furthermore, sampling higher diffusion times is a possibility, since correspondingly higher ES factors allow these to be acquired at the same TR (e.g. $430 \mathrm{~ms}$ at $\mathrm{ES}=6$ and $560 \mathrm{~ms}$ at $\mathrm{ES}=8$ ).

\subsection{Outlook}

As mentioned above, a useful extension might be a multi-echo EPI readout, which would enable further efficiency in mapping both $\mathrm{qT}_{1}$ and $\mathrm{qT}_{2} /$ $\mathrm{qT}_{2}{ }^{*}$ since unused duty cycle in optimal-TM sampling for $\mathrm{T}_{1}$ relaxometry can be used to sample multiple TE's per shot for $\mathrm{T}_{2} / \mathrm{T}_{2}{ }^{*}$ relaxometry. This could potentially also make use of efficient sparse sampling trajectories of the temporal relaxation curve, such as in echo-planar time-resolved imaging (EPTI; [222]). A multi-echo readout would also enable further speed-up of mixed or dependent contrast sampling, such as mixed $\mathrm{T}_{2} / \mathrm{T}_{2}$ * relaxometry and diffusion imaging [199, 216], and help characterise tissue compartments with separate relaxation constants and diffusion behaviour $[64,105,231]$. Future combination with SAR efficient MB pulses, such as PINS [155, 156], multi-PINS [75] and pTx-MB [232, 167] could allow high data quality at even higher MB factors. For the fastest acquisitions, acquisition of the reference data for GRAPPA and MB reconstruction takes a considerable proportion of the running time (about $30 \mathrm{~s}$ in the current implementation), which might be sped-up further with more efficient strategies. Additionally, a flexible table-based sequence looping implementation in which all parameters (TM, ES, TE, diffusion direction and gradient amplitude) can be controlled independently, could allow running $\mathrm{qT}_{1}, \mathrm{qT}_{2}$, $\mathrm{B}_{1}{ }^{+}$and diffusion mapping in a single sequence run with a shared reference scan and reversed phase-encode direction scans, further decreasing the overhead time. Finally, the SAR efficiency of a STEAM-based sequence 
makes it attractive for high duty cycle and multiband acceleration at $7 \mathrm{~T}$, but many of the advantages could translate well to $3 \mathrm{~T}$, particularly when MB factors are increased even further, as allowed by modern 32 to 64 channel head or head/neck RF-coils.

\subsection{Conclusion}

MESMERISED achieves super-accelerated 7 T STEAM imaging by combining echo-shifting and multiband/simultaneous multi-slice acceleration. This leads to very high multiplicative acceleration factors and time efficiency for $\mathrm{qT}_{1}$ and diffusion imaging. MESMERISED can probe combined $\mathrm{T}_{1}, \mathrm{~T}_{2}$ and diffusion contrast with high time efficiency for fast multicontrast mapping and characterisation of multi-component relaxometry, diffusion, and exchange.

\subsection{Acknowledgements}

AR and FJ were supported by an ERC Starting Grant (MULTICONNECT, \#639938). AR was further supported by a Dutch science foundation (NWO) VIDI Grant (\#14637). BP is partially funded by NWO VIDI Grant 016.Vidi.178.052 and NIH R01 MH111444/MH/NIMH. 


\subsection{Supplementary material}

\begin{tabular}{|c|c|c|c|c|c|}
\hline $\begin{array}{c}\text { Resolution - } \\
\text { slices }\end{array}$ & $\begin{array}{c}\text { MB - } \\
\text { GRAPPA }\end{array}$ & $T R[\mathrm{~s}] / \mathrm{TE}[\mathrm{ms}]$ & ES (TM[ms]) & ESXMB & $\begin{array}{l}\text { Acquisition time } \\
1 / 3 / 5 \text { reps [ } m: s]\end{array}$ \\
\hline $\begin{array}{l}1.8 \mathrm{~mm}- \\
72 \text { slices }\end{array}$ & $3-2$ & $6.90 / 28$ & $\begin{array}{c}\text { 1(140), } \\
2(280), \\
3(420), \\
4(560), \\
6(840), \\
8(1120), \\
12(1680)\end{array}$ & $\begin{array}{c}3, \\
6, \\
9, \\
12, \\
18, \\
24, \\
36 \\
\end{array}$ & $1: 18 / 2: 07 / 4: 32$ \\
\hline $\begin{array}{l}1.25 \mathrm{~mm}- \\
108 \text { slices }\end{array}$ & $3-3$ & $4.4 / 31.70$ & $\begin{array}{c}1(60), \\
2(120), \\
3(180), \\
4(240), \\
6(360), \\
9(540), \\
12(720), \\
18(1080) \\
\end{array}$ & $\begin{array}{c}3, \\
6, \\
9, \\
12, \\
18, \\
27, \\
36, \\
54 \\
\end{array}$ & $1: 05 / 2: 16 / 3: 26$ \\
\hline $\begin{array}{l}1.25 \mathrm{~mm}- \\
108 \text { slices }\end{array}$ & $3-3$ & $8.8 / 31.70$ & $\begin{array}{c}1(120), \\
2(240), \\
3(360), \\
4(480), \\
6(720), \\
9(1080), \\
12(1440), \\
18(2160)\end{array}$ & $\begin{array}{c}3, \\
6, \\
9, \\
12, \\
18, \\
27, \\
36, \\
54\end{array}$ & $1: 40 / 4: 01 / 6: 22$ \\
\hline
\end{tabular}

Figure 6.11: Quantitative $T_{1}$ imaging parameters. Total acquisition times are for 1,3 and 5 repetitions for each echo-shifting/mixing time (ES/TM) and with included 30 seconds of acquisition time due to GRAPPA and MB reference scans.

\begin{tabular}{|c|c|c|c|c|c|}
\hline $\begin{array}{l}\text { Resolution - } \\
\text { slices }\end{array}$ & $\begin{array}{c}\text { MB - } \\
\text { GRAPPA }\end{array}$ & $T R[\mathrm{~s}] / \mathrm{TE}[\mathrm{ms}]$ & $E S$ (TM[ms]) & $\begin{array}{c}T E[m s] \text { or } \\
\text { Flip angle } \\
\alpha_{T X}[\text { deg] }\end{array}$ & $\begin{array}{l}\text { Acquisition time } \\
1 / 3 / 5 \text { reps [m:s] }\end{array}$ \\
\hline $\begin{array}{c}1.8 \mathrm{~mm}- \\
72 \text { slices }\end{array}$ & $3-2$ & 5.2 /variable & $1(70)$ & $\begin{array}{l}\mathrm{TE}= \\
28.3, \\
38.3, \\
48.3, \\
68.3, \\
88.3\end{array}$ & $0: 56 / 1: 48 / 2: 40$ \\
\hline $\begin{array}{l}1.8 \mathrm{~mm}- \\
72 \text { slices }\end{array}$ & $3-2$ & $5.2 /$ variable & $3(420)$ & $\begin{array}{l}\mathrm{TE}= \\
28.3, \\
38.3, \\
48.3, \\
68.3, \\
88.3\end{array}$ & $0: 56 / 1: 48 / 2: 40$ \\
\hline $\begin{array}{l}1.8 \mathrm{~mm} \text { - } \\
72 \text { slices }\end{array}$ & $3-2$ & $6.9 / 28.3$ & $1(140)$ & $\begin{array}{c}\alpha_{x x}= \\
30, \\
50, \\
70, \\
90, \\
110\end{array}$ & 1:05/2:14/03:23 \\
\hline
\end{tabular}

Figure 6.12: Quantitative $T_{2}$ and $B_{1}{ }^{+}$imaging parameters. $T_{2}$-weighting imaging (row 1 and 2) uses standard Exc-Sto-Rec $=90^{\circ}-90^{\circ}-90^{\circ} . \mathrm{B}_{1}{ }^{+}$imaging (row 3) uses flip angle combinations of Exc-Sto-Rec $=\alpha^{\circ}-2 \alpha^{\circ}-\alpha^{\circ}$ for different multipliers $\alpha$. Total acquisition times are for 1,3 and 5 repetitions for each TE or flip angle combination and including 30 seconds of GRAPPA and MB reference scans. 
6 MESMERISED: Super-accelerated 7 T STEAM imaging for quantitative multi-contrast and diffusion MRI

\begin{tabular}{|c|c|c|c|c|c|}
\hline $\begin{array}{l}\text { Resolution - } \\
\text { slices }\end{array}$ & $\begin{array}{c}\text { MB - } \\
\text { GRAPPA }\end{array}$ & $T R[\mathrm{~s}] / \mathrm{TE}[\mathrm{ms}]$ & $E S(T M[m s])$ & $\begin{array}{c}b\left[s / \mathrm{mm}^{2}\right] \text { (\#dir) } \\
\text { STE echo }\end{array}$ & $\begin{array}{l}\text { Acquisition time- } \\
\mathrm{Nr} . \mathrm{vol}-\mathrm{sec} / \mathrm{vol}\end{array}$ \\
\hline $\begin{array}{l}2.0 \mathrm{~mm}- \\
60 \text { slices }\end{array}$ & $3-2$ & $2.36 / 40.4$ & $5(290)$ & $\begin{array}{c}\text { b0 (17), } \\
1750(24), \\
3500(36), \\
5250(48), \\
7000(60)\end{array}$ & $\begin{array}{c}7: 47 \\
370 \text { vols }-1.18 \mathrm{~s} / \mathrm{vol}\end{array}$ \\
\hline $\begin{array}{l}1.8 \mathrm{~mm}- \\
72 \text { slices }\end{array}$ & $3-2$ & $3.45 / 49.8$ & $2(140)$ & $\begin{array}{c}\text { b0 (17), } \\
1750(24), \\
3500(36), \\
5250(48), \\
7000(60)\end{array}$ & $\begin{array}{c}11: 08 \\
370 \mathrm{vols}-1.73 \mathrm{~s} / \mathrm{vol}\end{array}$ \\
\hline $\begin{array}{l}1.8 \mathrm{~mm}- \\
72 \text { slices }\end{array}$ & $3-2$ & $3.45 / 46.0$ & $3(210)$ & $\begin{array}{c}\text { b0 (17), } \\
1750(24), \\
3500(36), \\
5250(48), \\
7000(60)\end{array}$ & $\begin{array}{c}11: 08 \\
370 \text { vols }-1.73 \mathrm{~s} / \mathrm{vol}\end{array}$ \\
\hline $\begin{array}{l}1.8 \mathrm{~mm}- \\
72 \text { slices }\end{array}$ & $3-2$ & $3.37 / 40.8$ & $6(420)$ & $\begin{array}{c}\text { b0 (17), } \\
1750(24), \\
3500(36), \\
5250(48), \\
7000(60)\end{array}$ & $\begin{array}{c}10: 53 \\
370 \text { vols }-1.69 \mathrm{~s} / \mathrm{vol}\end{array}$ \\
\hline $\begin{array}{l}1.8 \mathrm{~mm}- \\
72 \text { slices }\end{array}$ & $3-2$ & $3.50 / 45.0$ & $\begin{array}{c}1(70), \\
2(140), \\
3(210), \\
4(280)\end{array}$ & $\begin{array}{c}\text { b0 (8), } \\
1000(24) \\
2500(48)\end{array}$ & $\begin{array}{c}16: 50 \\
560 \text { vols }-1.75 \mathrm{~s} / \mathrm{vol}\end{array}$ \\
\hline
\end{tabular}

Figure 6.13: Diffusion imaging parameters. Total acquisition times are for all combinations of ES/TM and b-values and including 30 seconds of GRAPPA and MB reference scans. Number of volumes in the final column is SE and STE volumes for all b-shells and ES/TM. B-values are listed for the STE echo volume.

\begin{tabular}{|c|c|c|cc|}
\hline ES & TM [ms] & $\boldsymbol{\delta}[\mathbf{m s}]$ & $\mathbf{4 / 4 ,} \mathbf{3 / 4}, \mathbf{2 / 4}, \mathbf{1 / 4}$ of $\mathbf{m a x}$ SE b-val \\
\hline 6 & 400 & 7.37 & $223,167,112,56$ & $\mathrm{~s} / \mathrm{mm}^{2}$ \\
\hline 4 & 280 & 8.65 & $350,264,176,88$ & $\mathrm{~s} / \mathrm{mm}^{2}$ \\
\hline 3 & 210 & 9.83 & $483,362,241,120$ & $\mathrm{~s} / \mathrm{mm}^{2}$ \\
\hline 2 & 140 & 11.71 & $752,564,376,188$ & $\mathrm{~s} / \mathrm{mm}^{2}$ \\
\hline
\end{tabular}

Figure 6.14: Combinations of echo-shift factor (ES), mixing time (TM), diffusion gradient length ( $\delta$ ), and spin-echo b-value achievable at the same TR for the case of $1.8 \mathrm{~mm}$ isotropic, maximum STE $b$-value $=7000 \mathrm{~s} / \mathrm{mm}^{2}$, at full duty cycle for GRAPPA factor 2 and multiband factor 3 . The listed SE b-values are for a 4 -shell acquisition with equally distributed b-values (i.e. at $4 / 4,3 / 4,2 / 4$ and $1 / 4$ of max-b), corresponding to STE $b$-value $=7000,5250,3500,1750$ $\mathrm{s} / \mathrm{mm}^{2}$. Note that full-duty cycle values also depend on the EPI readout train length, and therefore values would be slightly different for different resolutions and GRAPPA factors. 


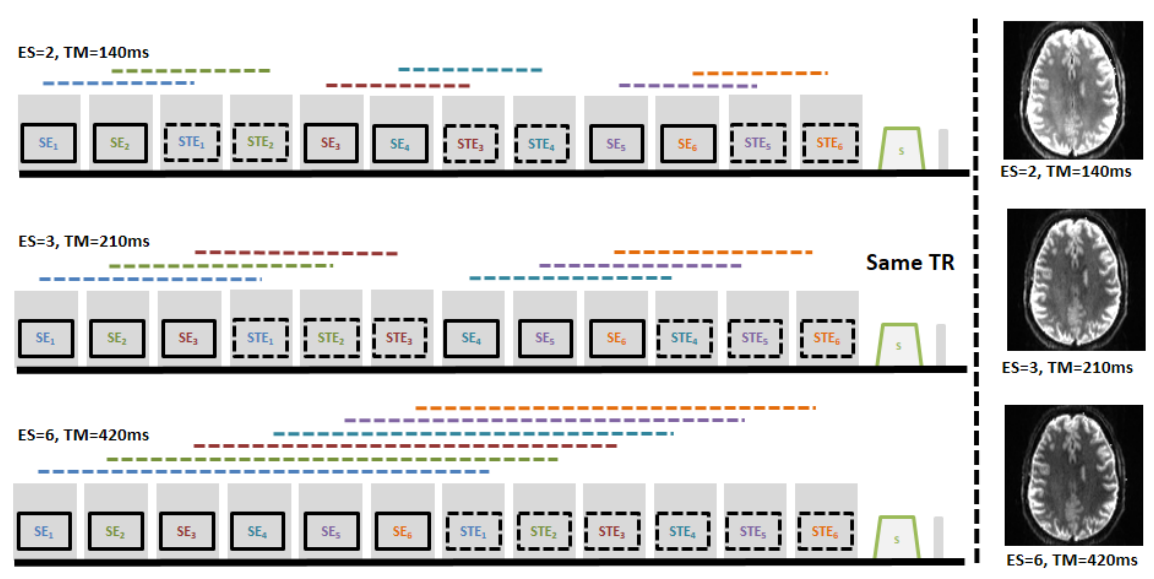

Figure 6.15: MESMERISED enables a multitude of mixing times at the same TR with full duty cycle. Here SE and STE signals are acquired for 6 slice groups, each containing the number of slices determined by the employed MB factor. Each sequence block containing ExcSto pulses and $\mathrm{SE}$ readout $\left(\mathrm{SB}^{\mathrm{A}}\right.$ in main Figure 6.1) is labelled as $\mathrm{SE}_{\mathrm{i}}$; each sequence block containing Rec pulse and STE readout $\left(\mathrm{SB}^{\mathrm{B}}\right.$ in main Figure 6.1) is labelled as $\mathrm{STE}_{\mathrm{i}}$. SE and STE readout for the same slice groups, as well as the time evolution of longitudinally stored magnetisation during the mixing time (dashed horizontal lines), are encoded by the same colour. In MESMERISED each echo-shift factor (ES factor) engenders a simple reordering of the same sequence blocks to enable different mixing times at the same TR. This TR is the same for all allowable TM's and is minimised by employing the full acquisition duty cycle. The illustrated examples are for sequence block length of $70 \mathrm{~ms}$, enabling TM $=140 \mathrm{~ms}$ for $\mathrm{ES}$ factor 2 (top), $\mathrm{TM}=210 \mathrm{~ms}$ for $\mathrm{ES}=3$ (middle) and TM $=420 \mathrm{~ms}$ for $\mathrm{ES}=6$ (bottom). 


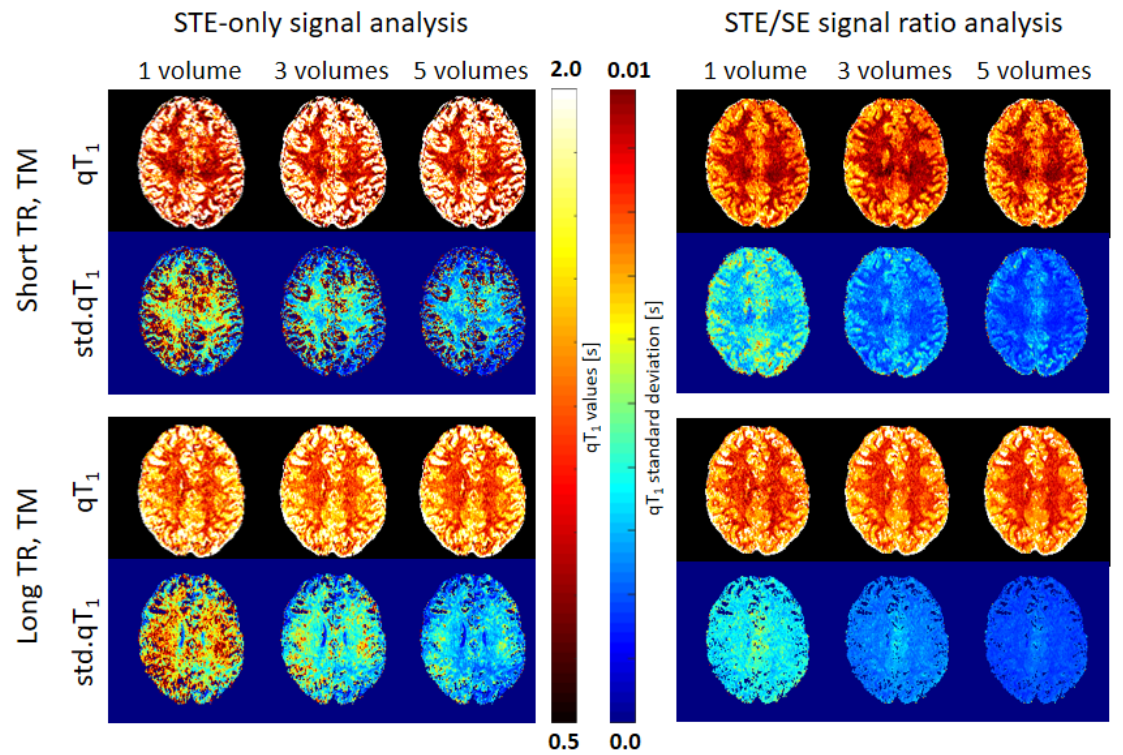

Figure 6.16: MESMERISED $1.25 \mathrm{~mm}$ isotropic $\mathrm{qT}_{1}$ mapping and its uncertainty compared between: short vs. long TR and TM (top vs. bottom), STE-only vs STE/SE ratio analysis (left vs. right) and number of repetitions (1, 3 and 5). Short TR is $4.4 \mathrm{~s}$ with a maximum TM of 1080 $\mathrm{ms}$, in which case 1/3/5 repetitions have an acquisition time of 1:05 / 2:16 / 3:26 respectively; long TR is $8.8 \mathrm{~s}$ with a maximum TM of $2160 \mathrm{~ms}$, in which case $1 / 3 / 5$ repetitions have an acquisition time of 1:40 / 4:01 / 6:22 respectively (c.f. Supplementary Table 6.11). STE-only $\mathrm{qT}_{1}$ analysis is performed with Equation 6.2, whereas $\mathrm{STE} / \mathrm{SE}$ ratio analysis is performed with Equation 6.5. Uncertainty or precision of the point-estimates is mapped as standard deviations derived from the Fisher Information Matrix. Uncertainty in $\mathrm{qT}_{1}$ estimates is lower 1) with increasing repetitions, 2) at long TR, TM compared to short TR, TM, and 3) with STE/SE ratio analysis compared to STE-only analysis. 
General Overview and Discussion 


\subsection{Overview}

Current medical imaging techniques (e.g. computed tomography, ultrasound, positron emission tomography, magnetic resonance imaging) have opened access for scientists and clinicians to a minimally (to non-) invasive study of the human body. Its anatomical, structural and functional characteristics can be unravelled using these techniques, which rely on properties such as electromagnetic (EM) signal attenuation, sound permeability, and magnetism. The relationship between the acquired signal and the underlying biological structures and processes has been a topic of discussion between researchers. There is a disagreement on which models to apply and which assumptions to make.

One of the conflict points is the relationship between the image resolution (spatial and/or temporal) and the resolution of the physical phenomena or biological process of interest. In the context of neuroscience, where the brain is the organ of interest, the use of quantitative MRI (qMRI) and diffusion MRI (dMRI) ${ }^{1}$ has brought forward valuable information about the brain macro and microstructure. The collected information from those techniques are aimed to validate the plausible connection between the magnetic properties like relaxation times (i.e. $\mathrm{T}_{1}$ and $\mathrm{T}_{2}$ from $\mathrm{qMRI}$ ) and water self-diffusivity $\left(\mathbf{D}_{\mathrm{j}}^{\mathbf{i}}\right.$, from dMRI), with the underlying biology of the brain, for example, the fibre distributions and/or configurations (as discussed in the Introduction (Chapter 2) of this thesis). However, MR techniques are amenable to the same discussions, especially in this plausible connection mentioned previously (a generalised overview in this topic was introduced in the special issue about Microstructural Imaging in NeuroImage [162]). One way to tackle this problem in neuroscience is by exploring and extending the analysis from the in vivo brain to the ex vivo brain. Investigation of ex vivo brains permits the acquisition of higher spatial resolution qMRI and dMRI images as shown in Chapters 3 and 4. The high spatial resolution images enable, in principle, the cross-validation between the estimated physical parameters and their corresponding biological structures and processes using $\mathrm{qMRI} / \mathrm{dMRI}$ and histology analysis, especially if they are performed on the same specimen [118]. If the correlation between the MRI contrast and the histological finding is significant,

\footnotetext{
${ }^{1}$ Actually, the concept of quantitative MRI introduced here and specially in the Quantitative MRI book [49] involves all the techniques in which a quantitative estimation of a physical parameters can be obtained. Under this definition, dMRI is intrinsically included into this qMRI concept. But in this work, qMRI will only involve relaxometry studies (e.g. $\mathrm{T}_{1}$ and $\mathrm{T}_{2}$ ) and be separated from dMRI.
} 
then the assumed biological interpretation assigned to the qMRI/dMRI results can be considered valid. And therefore, a proper extension of those results can be applied to the corresponding $\mathrm{qMRI} / \mathrm{dMRI}$ results obtained using in vivo specimens. This extrapolation, from ex vivo to in vivo, must at least include, the biological differences between both specimens (as mentioned in Introduction (Chapter 2), and in the next references [71, 175]). To achieve the mentioned cross-correlation between MRI and histology, it is desirable to achieve mesoscale spatial resolution (much lower than 1000 $\mu \mathrm{m}$ isotropic) in qMRI/dMRI, which would require the use of powerful gradients [118], ultra-high $\mathbf{B}_{0}{ }^{i}$ field strengths [125, 211] and optimised MRI sequences [203]. Taking into consideration all the advantages and disadvantages in the use of powerful MRI hardware [118, 125, 211], sequence optimisation becomes a vital component since a proper optimisation would allow achieving the highest SNR/resolution possible for any hardware configuration [118].

The main goal of this thesis, then, was to show how some sequences were optimised for ex vivo and in vivo qMRI and dMRI studies in ultra-high field scanners (from $7 \mathrm{~T}$ and above).

\subsubsection{MRI pulse sequence optimization}

In Chapters 3, 4 and 6 optimised MRI sequences were presented for qMRI and dMRI in UHF MRI scanners. While the first two chapters were aimed at imaging acquisition in ex vivo human brains (performed at 9.4 T), the latter was aimed at ultra-fast in vivo human brain imaging (performed at 7 T). Chapter 3 introduced the $\mathrm{k}_{\mathrm{T}}$-dSTEAM sequence and Chapter 4 introduced the $\mathrm{k}_{\mathrm{T}}$-dSSFP sequence. Both were optimised to solve the complex $\mathbf{B}_{1}{ }^{i}+$ spatial inhomogeneity present in UHF scanners in human brain sized samples [211] by using a sophisticated RF pulse design using the $\mathrm{k}_{\mathrm{T}}$-points method [53]. Both sequences were shown to be SNR-advantageous on UHF human bore MR scanners for dMRI ex vivo acquisitions (in terms of SNR and flexibility) in comparison to the gold-standard diffusion-weighted pulsed gradient spin-echo (PGSE) sequence (see Figure 3.5 and reference [84]) With this SNR advantage, $\mathrm{k}_{\mathrm{T}}$-dSTEAM acquired homogeneous multicontrast (multi-shell diffusion-weighted and relaxometry-weighted) images from high $(1000 \mu \mathrm{m})$ to ultra-high isotropic resolution $(400 \mu \mathrm{m})$. Through the analysis and results of the diffusion datasets, ultra-high resolution data showed advantages in tractography analysis, whereas high resolution multi-shell varying TM data allowed establishing dMRI acquisition 
protocols which maximised SNR at each b-value shell (c.f Supplementary Figure 3.14). The SNR shown in $\mathrm{k}_{\mathrm{T}}$-dSSFP images allowed achieving accelerated ultra-high $450 \mu \mathrm{m}$ and $500 \mu \mathrm{m}$ isotropic resolution whole brain dMRI data at moderate b-values $\left(3000-4000 \mathrm{~s} / \mathrm{mm}^{2}\right)$. The performed spinecho diffusion tensor imaging (SE-DTI) analysis on this data showed an increased contrast between white matter and grey matter in both resolutions. However, the highly complex signal model for the dSSFP signal and its dependency on parameters like $\mathrm{T}_{1} / \mathrm{T}_{2} / \mathbf{B}_{1}{ }^{\mathrm{i}}+$ make this sequence less suitable for quantitative microstructure studies (in comparison to diffusionorientation studies $[143,144])$. In this context, the use of $\mathrm{k}_{\mathrm{T}}$-dSTEAM or $\mathrm{k}_{\mathrm{T}}$-dSSFP in this type of research necessarily depends on the application, scanner capabilities and time constraints.

Chapter 6 introduced the MESMERISED sequence. This sequence is based on the STEAM sequence and optimised to perform very highly accelerated acquisitions for in vivo studies (from x 4 to even x 54 times total acceleration; from approximately 2 to 9 times faster than multiband-only accelerated acquisition). This was achieved by applying an echo-shifting (ES) approach during long TM periods in STEAM, resulting in additional acquisitions during what would normally be dead time. This, combined with multiband/simultaneous multi-slice (MB-SMS) RF pulses, makes the total acceleration factor proportional to ESxMB. The ES approach in MESMERISED does not introduce any extra signal penalties (like the geometric factor, or g-factor [35, 4] and signal attenuation) in the resulting spinecho (SE) and stimulated echo (STE) signals. The main advantage of this technique is the highly accelerated acquisition speed for time-consuming STEAM techniques like $\mathrm{T}_{1}$-weighted relaxometry and diffusion imaging at long diffusion times or high b-values. Besides, MESMERISED inherits the advantages of the STEAM imaging technique, such as its versatility for qMRI (including quantitive mapping of $\mathbf{B}_{1}{ }^{i}+, \mathrm{T}_{1}$ and $\mathrm{T}_{2}$ ) and dMRI techniques (including diffusion at varying TM with the same b-value, and varying $b$-value with the same TM). Resolutions of $2.0,1.8$, and $1.25 \mathrm{~mm}$ isotropic images at proportional ESxMB factors with a fixed reduced repetition time (TR) were acquired to show the performance of MESMERISED at different contrasts and SNR's. The outstanding amount of data per unit time (one full brain image at $1.8 \mathrm{~mm}$ isotropic in approximately 1.7 seconds per volume) that can be obtained through this sequence allows performing complex data analyses with data-hungry models (especially in dMRI) such as CHARMED [8], or combined contrast models, such as combined $\mathrm{qT}_{1}$ and diffusion microstructure modelling. Moreover, this sequence acquires the SE signal simultaneously, which is low-b diffusion-weighted (lower than 
$1000 \mathrm{~s} / \mathrm{mm}^{2}$ ), useful for analyses like DTI and intravoxel incoherent motion (IVIM) [126].

\subsubsection{Signal modelling}

Chapter 5 revisited the physics of the STEAM sequence through the exploration of the signal behaviour in the short TR regime. It was necessary to develop and to present a full expression for the longitudinal component (hereafter, steady-state) in the SE and STE signal equations, which is not usually explicitly defined for all TR regimes (e.g. in reference [7] introduced a reduced version only valid in the long $\mathrm{TR}$ regime, where $\mathrm{TR}>\mathrm{T}_{1}$ ). A generalised expression was then obtained and a comprehensive analysis was performed on the behaviour of SE and STE signals with the steadystate component properly inserted into the contrast evolution. First, the results verified that the flip angles for all the RF pulses in STEAM scale the signal, maximising it in most cases when the excitation, storing and recalling flip angles are $\pi / 2 \mathrm{rad}$ (as mentioned in the reference [85]), however with some exceptions. Second, the results demonstrated the possibility of obtaining a set of TM-TR combinations where the SNR can be maximised, and the contrast of a specific tissue (with a specific $\mathrm{T}_{1}$ ) can be maximised or minimised (achieving suppression of e.g. fluid) in comparison to the signal coming from other $\mathrm{T}_{1}$-components. This signal model was validated through the comparison of the modelled SE signal behaviour with the experimental SE data from a homogeneous phantom and an inhomogeneous ex vivo whole-brain specimen. The signal behaviour from experimental data showed a high correlation with the modelled signal for both study samples. The proposed signal model for SE and STE in the short TR regime can be also extended to different TR regimes.

\subsection{Discussion}

\subsubsection{Why was the STEAM sequence chosen in this work?}

First, STEAM is a sequence that is not as extensively explored and studied for ex vivo (and in vivo) imaging at UHF scanners as compared to PGSE (for example, see the work of the Human Connectom Project group, e.g. in reference [220]) and (arguably) SSFP (for example, see the research of 
Dr. Miller's group, e.g. references [146, 84]) sequences. This work helped further establishing it as a flexible and advantageous sequence for qMRI and dMRI studies. From the flexibility side, STEAM can be used for relaxometry $\left(\mathrm{T}_{1}, \mathrm{~T}_{2}\right)$, multi-parametric diffusion (same/different b-values at different/same diffusion times, or TM's) and relative field mapping (i.e. $\left.\mathbf{B}_{1}{ }^{i}+\right)$ with straightforward signal modelling. All techniques in this thesis have been explored in the context of UHF in vivo (Chapter 6) and ex vivo (Chapter 3). Furthermore, the double echo (SE and STE) acquisition approach, when feasible, increases the amount of information and analysis that can be performed. STEAM can also overcome some of the disadvantages experienced by other sequences, as previously discussed in the aforementioned chapters.

However, this sequence has inherent drawbacks that were considered and studied in this work, coming predominantly from the signal and acquisition time perspectives. From the signal perspective, STEAM loses half of the total signal in the STE signal alone. Recently, the use of the simultaneously acquired SE signal for complementary analysis purposes is becoming more common (as for example in $\mathbf{B}_{1}{ }^{i}+$ mapping [138] and shown in Chapter 6). In this thesis, the option of using this signal for analysis purposes was evaluated. It was necessary to understand the implications of acquiring this signal and its possible interference with the STE signal in a broad range of timing and flip angle conditions (Chapter 5). Subsequently, this knowledge was practically applied by obtaining results from stand-alone SE $q M R I$ and dMRI analysis (i.e. $\mathrm{T}_{2}$ and diffusion analysis in Chapter 6). The results from combined SE and STE analysis enriches the total biophysical information that can be obtained from this sequence, but only if the proper signal behaviour is considered (this is discussed further in the next subsection). From the acquisition time perspective, STEAM requires long time-consuming experiments when TM is employed as a variable parameter. As explored in Chapter 3 and Chapter 6, $\mathrm{T}_{1}$ relaxometry requires TM sampling values at least equal to or (preferably) considerably longer than $\mathrm{T}_{1}$ (for example, for in vivo white matter, at $7 \mathrm{~T}$, this is approximately $1.5 \mathrm{~s}[160,223])$. For dMRI experiments, if the b-value is predominantly dependent on TM (i.e. fixed maximum gradient amplitude and relatively short duration, $\delta$, to keep TE as short as possible), then high b-values require from moderated to long TM values (in Chapter 3, and Chapter 6). In each of those cases, TR must be even longer (at least TM plus readout gradient and TE/2, c.f. Figure 2.7). As a consequence, considerable dead periods are introduced, which are not reduced by the current (in-plane or simultaneous multi-slice) acceleration methods. Therefore, the implemen- 
tation of the ES acceleration (for MESMERISED in Chapter 6) was the immediate solution which uses the dead time to excite and acquire extra slices.

\subsection{Which information can be extracted from STEAM qMRI and dMRI data?}

As extensively shown in this work, the two main echoes (SE and STE) in STEAM can be used for both $\mathrm{qMRI}\left(\mathrm{q} \mathrm{T}_{1}\right.$ and $\mathrm{qT}_{2}$ ) and dMRI. Understanding how those echoes are generated can help to give an appropriate biological interpretation of their relaxometry and diffusion signal decay. Even though the following analysis will discuss each MRI technique independently, the SE and especially the STE are multi-contrast signals (i.e. $\mathrm{T}_{2}-\mathbf{D}^{\mathrm{i}}{ }_{\mathrm{j}}$ and $\mathrm{T}_{1}-\mathrm{T}_{2}-\mathbf{D}^{\mathrm{i}}{ }_{\mathrm{j}}$, respectively).

From a qMRI perspective, as described in reference [68], $\mathrm{T}_{1}$ and $\mathrm{T}_{2}$ (and other) parameters can reveal biological features in the brain. This depends, evidently, on the relaxometry signal modelling and the spatial resolution of the qMR images. The signal equations (explicitly described in Chapter 5) show that both signals (SE and STE) are differently $\mathrm{T}_{1}-\mathrm{T}_{2}$ weighted, as presented in Figure 7.1. Due to this weighting difference between signals, taking the ratio between them (STE/SE) can leave only the $\mathrm{T}_{1}$-relaxation phenomena contribution (as shown in 6). This is useful to avoid the inherited model degeneracy for $\mathrm{T}_{1} \mathrm{STE}$ relaxometry estimation, as discussed in Chapter 5. On the other hand, both signals are equally weighted by $\mathrm{T}_{2}$, and similarly as in PGSE. It is clear then, that quantitative $\mathrm{T}_{2}$ mapping can be performed with only the SE or STE signals, or both simultaneously (i.e. as in Chapter 6). It should be noted in Figure 7.1 that the effect of the $\mathrm{T}_{1}$ weighting in STE decreases the different signal decay steepness in comparison to $\mathrm{SE} \mathrm{T}_{2}$ signal decay. 


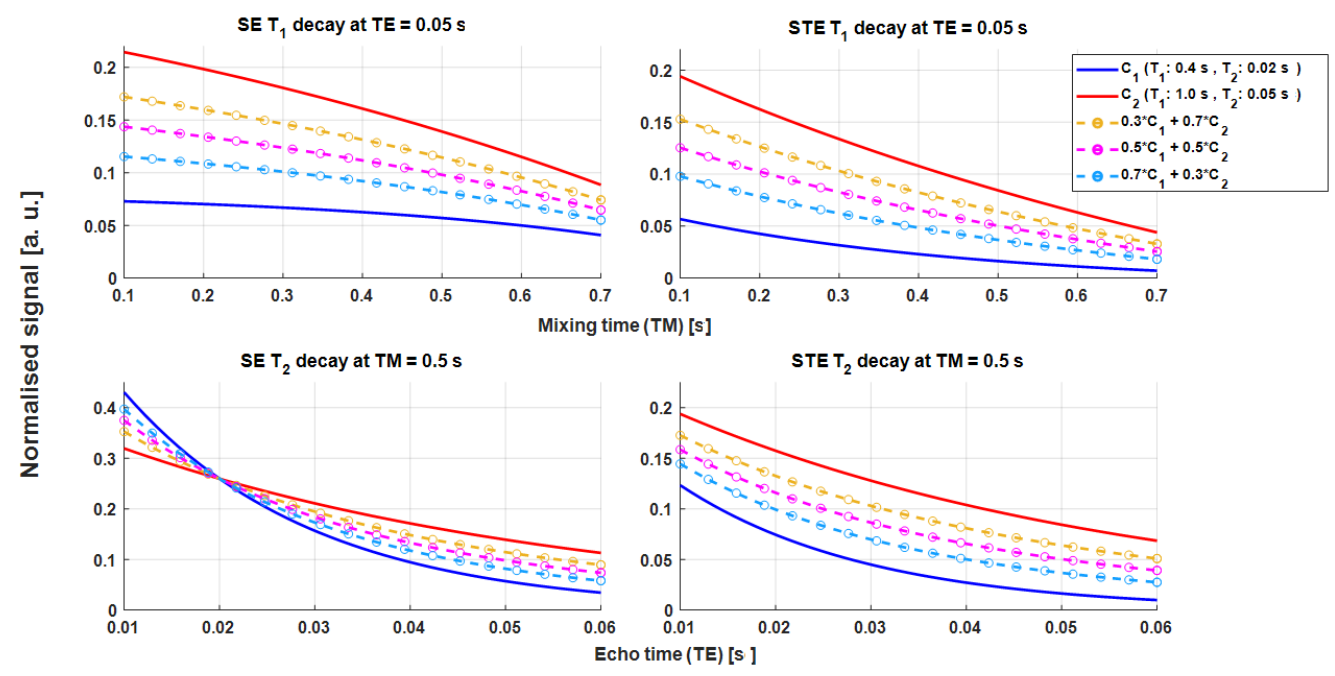

Figure 7.1: $\mathrm{SE}$ and STE signal decays (first and second column, respectively) due to relaxometry ( $\mathrm{T}_{1}$, top row and $\mathrm{T}_{2}$, bottom row) at different TM's and TE's for two signal compartments $\left(\mathrm{C}_{1}\right.$ and $\left.\mathrm{C}_{2}\right)$ independently (solid lines) and sum-weighted (dashed lines). The signal equations employed are the simplified versions of Equations 5.1, 5.2 and 5.14 in Chapter 5. For $\mathrm{T}_{1}$ relaxometry, both signals decay differently, where the SE decays as a function of the total signal available per compartment and the STE decays as a function of the total signal and relaxometry phenomena. For $\mathrm{T}_{2}$ relaxometry both signals decay equally but the extra contribution of T1 in the STE signal reduces the steepness of the $\mathrm{T}_{2}$ signal decay contribution for each compartment.

This differentiation property of the STE signal can be exploited to disentangle the differential contribution from different brain structures, given the fact that the $\mathrm{T}_{1}$ and $\mathrm{T}_{2}$ values for the extra-axonal, myelin and intraaxonal water molecules are different (e.g. see references $[48,68]$ ). On the other hand, from a dMRI perspective, (as described in references [71, 128]), the microstructural information is more sensitive to time diffusion parameters (i.e. $\Delta$ and $\delta$ ) than gradient amplitude $\left(\sqrt{\mathbf{G}_{i} \mathbf{G}^{i}}\right)$. Experimentally it has been shown that under the same b-value at different $\Delta$, the signal decays differently, mostly influenced by the geometry of the compartments containing the spins (e.g. cylinders in the case of axons) [83, 128]. In STEAM, acquiring variable diffusion times in dMRI can be performed by varying TM in the STE signal. This results in higher SNR images at high b-value, making them suitable for diffusion analysis using complex diffusion models. Moreover, given the structure of the sequence (Figure 2.7), 
the $\mathrm{SE}$ signal is also $\mathrm{T}_{2^{-}}$and diffusion-weighted, although much less (as studied in Chapter 6). In this context, stand-alone analysis of the SE and STE diffusion-weighted signals can extend the understanding of the signal sources. It is known in literature how the different biological components are sensitive to $b$-value ${ }^{2}$. For example, low b-values $\left(<500 \mathrm{~s} / \mathrm{mm}^{2}\right)$ are sensitive predominantly to the coherent and incoherent fluid motions in vasculature [126]. Moderate high b-values $\left(\geq 1000 \mathrm{~s} / \mathrm{mm}^{2}\right)$ are sensitive to a more global diffusion perspective (in other words, only diffusion phenomena are measured), predominantly in the extra-cellular (where most of the signal comes from) and intra-cellular compartments. Finally, high b-values (beyond $7000-8000 \mathrm{~s} / \mathrm{mm}^{2}$ ) are predominantly sensitive to intracellular diffusivity since the signal coming from the extra-cellular compartment is fully suppressed (therefore, $\mathbf{D}_{\text {jintra }}<\mathbf{D}_{\text {jextra }}^{\mathbf{i}_{\text {}} \text { ) }}[216,159,117]$. Therefore, it would be advantageous to develop proper signal modelling that incorporates the SE and STE signals simultaneously. In this way, each modelled compartment can be described more precisely as a function of its own relaxometry and diffusion parameters.

As a whole, considering the multi-contrast properties of STE and SE signals, it is clear that performing dMRI with STEAM comes with relaxometry phenomena that cannot be avoided. As extensively studied in Chapter $\mathbf{5}$, it is possible to choose sequence parameters (TR and TM, but also TE) that can suppress or enhance specific signal components (by the steadystate component). If those are simultaneously weighted by diffusion then proper signal discrimination from different signal components can be performed. For example, diffusion models where a different relaxometry component (i.e. $\mathrm{T}_{2}$ ) per signal compartment is assigned have already been proposed (e.g. see references [216,63]). This allows discriminating whether the diffusion anisotropy of the water molecules come from the intra or extra-axonal compartment, as well as estimating the g-ratio ${ }^{3}[194,46]$. The benefits of this discrimination could be used for tractography, where the definition of streamlines can not only be based on the water self-diffusion orientation but also on other microstructural information (e.g. g-ratio, or relaxometry values $[67,58]$ ) The correlation between diffusion anisotropy and quantitative parameters has been extensively studied (see reference [48]), showing that the anatomical information that can be extracted from

\footnotetext{
${ }^{2}$ The following values are reported for the in vivo situation. For ex vivo specimens, the values are approximately twice as high and evidently, some biological compartments are not present i.e. vasculature

${ }^{3}$ G-ratio is defined as the ratio of the inner to the outer radius of the myelin sheath of a myelinated axon
} 
any brain specimen (in vivo or ex vivo) requires proper understanding of both contrasts. This is illustrated with $\mathrm{k}_{\mathrm{T}}$-dSTEAM data of an ex vivo human occipital lobe in Figure 7.2 (and in reference [86]). A visible contrast in grey matter (GM) and white matter (WM) for both parameters is noticeable and likely represents the influence or concentration of different elements, like myelin, iron, or even the fibre orientation with respect to the main magnetic field [48, 68, 227].

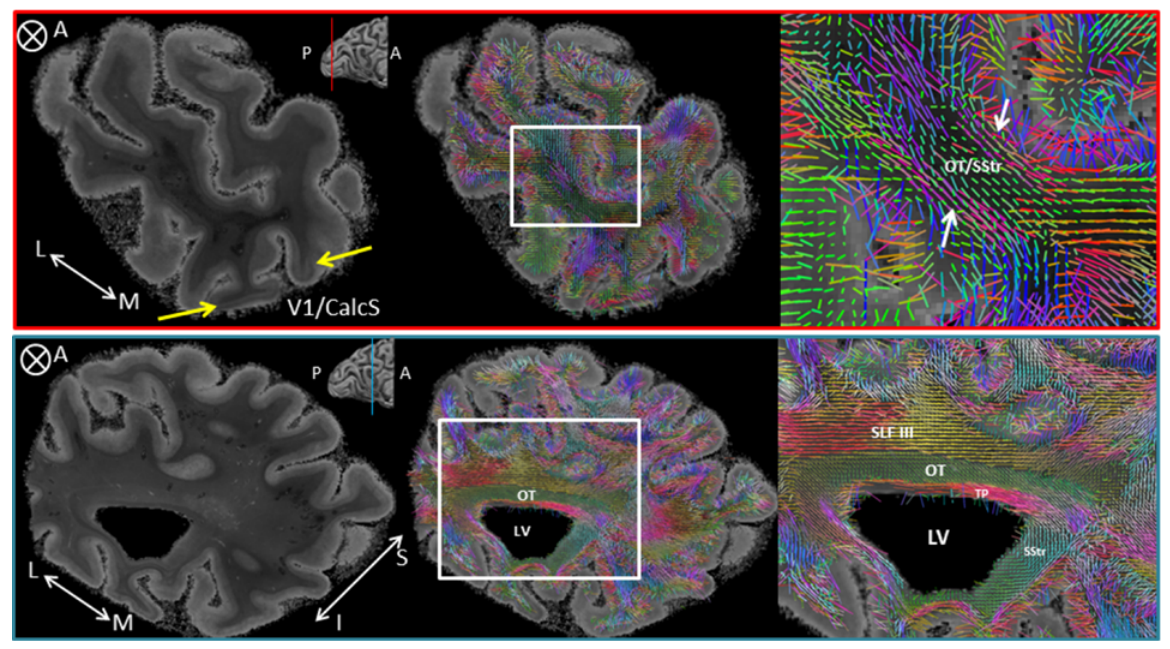

Figure 7.2: Ex vivo occipital lobe $200 \mu \mathrm{m}$ relaxometry and overlayed $400 \mu \mathrm{m}$ main diffusion direction from diffusion MRI study on the same specimen [86]. By using a gradient-echo (GRE) sequence (for $q M R I$ ), it is possible to estimate $\mathrm{T}_{1}\left(\mathrm{qT}_{1}\right.$, not shown) and effective $\mathrm{T}_{2}$ ( $\mathrm{qT}_{2}{ }^{*}$, shown here), while with $\mathrm{k}_{\mathrm{T}}$-dSTEAM it is possible to estimate the main diffusivity directionality. Not only the contrast between WM/GM is enhanced in the $\mathrm{qT}_{2} *$ map (first row), but also into the WM. The orientation of the main diffusion vector is related with the $\mathrm{WM} \mathrm{qT}_{2} *$ constrast [227]. V1/CalcS: Visual area 1 and Calcarine Sulcus, SLF III: Superior Longitudinal Fasciculus III, OT: Optical Tract, TP: Tapetum, SStr: Sagital Stratium and LV: Left Ventricle.

\subsubsection{Outlook and promising future developments}

The work aimed for the optimisation of dMRI and qMRI sequences (predominantly STEAM) and their application for in vivo and ex vivo human brain acquisitions using UHF MRI human bore scanners. In the context of the STEAM sequence, several of its drawbacks were mitigated or minimised and the characteristic potential enhanced. However, there are fur- 
ther developments to be investigated in STEAM specifically, and with other pulse sequences more generally, which are presented in the following section.

First, regarding the MRI pulse sequences (SSFP, STEAM and PGSE) and their usage in ex vivo and in vivo research. For ex vivo studies, the MR sequences should be capable of acquiring high to ultra-high resolution images with enough SNR for analysis, to be analytically simple and to be time-efficient. With SSFP, time-efficiency is not a concern in comparison to the other sequences, but its signal modelling is severely dependent on quantitative maps (i.e. $\mathbf{B}_{1}{ }^{i}+, \mathrm{T}_{1}$ and $\mathrm{T}_{2}$ ). Therefore, using the SSFP sequence demands a robust protocol for acquiring and estimating those maps for quantitative diffusion imaging. Complementary to this, beyond the scope of this work, this sequence could prove valuable in the implementation of multi-shell diffusion models and analysis [202] ${ }^{4}$, together with a proper biological interpretation. In the case of STEAM, time efficiency and noise reduction (which is more predominant than in SSFP) are the critical points to be discussed. Regarding the time efficiency, advanced readouts and sophisticated undersampling approaches can be implemented. An extensive set of options are discussed in the next subsection ${ }^{5}$. Regarding the noise reduction, it is important to remark that it was considered but better approaches are desired to ease the high resolution-SNR trade-off, as shown in Chapter 3. For example, all the images were reconstructed with the root-of-covariance-sum-of-squares (rCovSoS), which helped in reducing background noise. Techniques like SENSE-1 reconstruction [187] or by a matrix theory denoising approach [215] could be used.

Besides the points discussed for individual sequences, there remain more general issues that must be studied and accounted for in both sequences: (a) image distortions and $\mathbf{B}_{1}{ }^{i}+$, and (b) temperature stability. In the case of point (a), ex vivo imaging suffers less imaging artefacts in comparison to in vivo studies, but it does not mean that those image distortions can be neglected. For example, severe magnetic susceptibility difference between tissue-solution or tissue-air bubbles introduces signal and geometrical distortions [183]. Furthermore, the high field inhomogeneity $\left(\mathbf{B}_{0}{ }^{i}\right)$ present in UHF produces severe phase offsets and frequency drifts [233] which requires sophisticated correction methods like conjugate phase correction (i.e. see review in reference [176]). Moreover, it has been shown that the

\footnotetext{
${ }^{4}$ This work got published during the writing period of this thesis

${ }^{5}$ It is important to remark that those options are not only limited to STEAM, but can be equally applied for PGSE and SSFP sequences.
} 
main magnetic field can suffer from instability through slow drift during long scan acquisitions. This can introduce a variation in $\mathbf{B}_{1}{ }^{i}+$ (and $\mathbf{B}_{0}{ }^{i}$, as already mentioned). Therefore, acquiring RF-maps in between measurements (by using DREAM [152] or Actual Flip Angle Imaging (AFI) [234] acquisitions) or RF signal during acquisitions (by using FID navigators, for example in reference [221]) can help in reducing this variation. In the case of point (b), it has been reported that temperature variation during scanning introduces a severe bias in some quantitative parameters like $T_{1}$ [171] and $\mathbf{D}_{j}{ }_{j}$ [104]. It has been established that performing dummy scans (from one to several hours) allows the ex vivo specimen to reach thermal equilibrium (which was applied in this work). However, this method is not entirely reliable because: (1) it does not ensure that a thermal equilibrium has been reached and (2) the use of hours for this method severely constraints the time for data acquisition. Therefore, it is proposed to establish an MR thermometry protocol that can be used interleaved with qMRI/dMRI ex vivo acquisitions [171] such as interleaving diffusion measures for ADC calculation in the solution [104, 171].

For in vivo human brain experiments, pre-processing (in the image space) is considered a priority. In this work, an established pre-processing pipeline was used (from the Human Connectome Project - HCP - Consortium [91], which includes motion correction, field map $\mathbf{B}_{0}{ }^{\mathrm{i}}$ correction and eddy current correction) and also included a denoising approach [215]. However, improved methods could be considered and might require additional acquisitions and the use of sophisticated image correction for motion (e.g. realtime prospective motion correction (PROMO) [228]), distortions (e.g. echo planar time-resolved imaging (EPTI) [222]), Gibbs ringing [214] and eddy currents (e.g. eddy current and motion correction (ECMOCO) [149]) or by using higher-order corrections [16]. Some of these have already been combined recently in updated dMRI preprocessing pipelines (e.g. DESIGNER [1]). The discussed points for ex vivo studies (i.e. high resolution, time efficiency and SNR) are also important for in vivo studies. However, there is a natural barrier that limits the use of the scanner for in vivo subjects: restrictions in SAR and peripheral nerve stimulation establish a borderline, which does not exist for ex vivo specimens.

Regarding readout advances and accelerated readouts, it has been reported several promising techniques that allow faster data acquisition while keeping or even improving data quality. For all the discussed sequences (for ex vivo, $\mathrm{k}_{\mathrm{T}}$-dSTEAM - $\mathrm{k}_{\mathrm{T}}$-dSSFP; and in vivo, MESMERISED) some of those techniques could be implemented as follows: for accelerated ultra-high resolution 3D acquisitions (i.e. for ex vivo studies), three dimensional (3D) 
CAIPIRINHA [34] and spiral readout [31] can be used. Whereas the first approach could allow performing higher resolution images with a shorter total acquisition time, the second approach not only decreases total acquisition time but also improves SNR due by using a shorter TE's. For accelerated 2D acquisitions (i.e. for in vivo studies), blip up-down acquisition (BUDA or DR-BUDDI) [106] and EPTI techniques can be used. In contrast to the first-mentioned techniques, these methods can help in acquiring images with less distortion and blurring by optimising subspace reconstruction (in the case of BUDA) or acquiring interleaved oppositepair (e.g. blip-up and blip-down) encoding lines (in the case of EPTI). All the aforementioned techniques (and others) require sophisticated reconstruction procedures to obtain the desired images.

Regarding the multi-contrast imaging and multi-parametric microstructure modelling shown for the STEAM sequence (Chapter 5) is far from complete. It is indeed promising to see that the SE and STE data can be used jointly (as shown in Chapter 6), but with the risk of falling in degeneracies, as extensively discussed in the works of Does et al. 2018 [68] and Novikov et al. 2018 [158]. A degeneracy, as meant in the context of the signal fitting, is where a large set of model parameter values will all fit the data equally, making interpretation of any specific values problematic. Therefore it is suggested, by the dMRI community for example, to establish a careful acquisition sampling (with variable timing parameters and amount of data) which can be used with improved signal models (as discussed in the reference [48]). On the other hand, the incorporation of the $\mathrm{SE}$ signal from $\mathrm{k}_{\mathrm{T}}$-dSTEAM for analysis can be beneficial for relaxometry rather than diffusion. This is because the low diffusion-weighting in the $\mathrm{SE}$ signal becomes even lower (if not negligible) in the context of ex vivo imaging.

Regarding MESMERISED (Chapter 6), it is possible to speed up the sequence even further by using multi-echo, EPTI or spiral-out readouts instead of echo-planar (EPI) readouts (as presented in the previous subsection). This allows faster acquisitions and higher data rates (especially in the case of multi-echo and EPTI) and shorter TE's (especially in the case of spiral-out). Besides, it could be advantageous to take advantage of of a $7 \mathrm{~T}$ pTx system, with PINS, multiPINS or pTX-MB pulses [155, 156], where $\mathbf{B}_{1}{ }^{i}+$ homogeneity can be improved and, as a consequence, achieve higher homogeneity of SNR and contrast over the image. Due to the potential of this sequence, its implementation for lower fields (e.g. $3 \mathrm{~T}$ ) can benefit quantitative MR research for clinical studies. It is noted that MESMERISED is already capable of achieving acceleration factors up to x 54, 
and speed-up up to $\mathrm{x} 9$ compared to multiband accelerated acquisitions (the current state-of-the-art in EPI imaging speed) and it could appear to be unrealistic to accelerate this sequence even further. However, first the imaging speed comes at the cost of SNR per image (starting from half the SNR of other sequences in STEAM) and the achievable spatial resolution. Second, the real objective of optimising this sequence (and others) is not merely speeding things up but also in allowing the acquisition of a large number of MR images (especially in in vivo studies) at reasonable resolutions with enough SNR for analysis. Then, biological microstructure features can be more easily discovered [159] and data-expensive signal models can be employed (like AxCaliber [12] or ActiveAx [70]). This is an endeavour where functional MRI is rapidly progressing, and it is where qMRI and dMRI should go as well.

\subsection{Final general remarks}

To summarise, the STEAM sequence allows acquiring insightful MR images in which the signal can be used to reveal physical and biological information from the subject or specimen under study. From a general perspective, the use of any sequence can show new insights (or, in a colloquial way, spark joy) with the correct acquisition, signal modelling, and interpretation. Conversely, it can cloud the research question in the void of image and signal analysis degeneracy, uncertainties, and false positives. The aim of this work was, from this point of view, to give an impulse to the use of the STEAM sequence, not as a competitor of other sequences, but as a complementary sequence that can contribute to the MRI community another option within its acquisition and analysis approaches. Therefore, it was necessary to progressively approach the different aspects of this sequence and steadily step through their analysis. Other acquisition methods (as mentioned previously), pre-processing and post-processing studies (such as tractography) were not fully covered in this work but it is necessary to investigate them. Future research might benefit from the reported improvements in the investigation of brain microstructure incorporated in this work, and from their further application to the entire human body. 


\section{Valorisation}


Knowledge valorisation: the process of creating value from knowledge, by making knowledge suitable and/or available for social (and/or economic) use and by making knowledge suitable for translation into competitive products, services, processes and new commercial activities. From Regulations governing the attainment of doctoral degree, 22, Maastricht University, NL.

\subsection{Research}

The main goal of this methodological thesis was to present optimised magnetic resonance imaging (MRI) sequences (steady-state free precession, SSFP; and predominantly stimulated echo acquisition method, STEAM) for ex vivo and in vivo quantitative MRI (qMRI) and diffusion-weighted MRI (dMRI) studies in ultra-high field scanners (UHF, from $7 \mathrm{~T}$ and above). The advantages of STEAM, severely constrained due to hardware limitations (e.g. $\mathrm{B}_{1}{ }^{+}$inhomogeneity) and sequence drawbacks (e.g. timeexpensive), were overcome. It resulted in ultra-high resolution wholebrain dMRI data for ex vivo studies (achieving $400 \mu \mathrm{m}$ isotropic resolution) using $\mathrm{k}_{\mathrm{T}}$-dSTEAM, and very highly accelerated $\mathrm{qMRI}$ and dMRI acquisition protocols for in vivo studies (up to $\mathrm{x} 54$ times the current total acceleration with STEAM; or up to 9 times faster than multiband-only accelerated acquisition) using MESMERISED. With the current feasibility of acquiring ultra-high resolution data and highly sampled multi-contrast MRI images, data-expensive signal models can be employed (e.g. for dMRI, like AxCaliber [12] or ActiveAx [70]) and tractography analysis can benefit; but above all, the biological microstructure features in the brain can be easily more revealed from the resulted analysis [159].

\subsection{Relevance}

From a scientific point of view, the optimisation of MR sequences was achieved to enable whole human brain acquisitions (either for ex vivo or in vivo studies). These MR images can provide information regarding the entire white matter network systems, the entire grey matter areas connected through these systems, as well the underlying biological microstructure. The concept of visualising biological structures and functions through MRI is defined in the MR community as in vivo MR histology (see the review of 
Bridge et al. 2006 [37]). This information, which could previously be accessible only through post mortem histology, would help to understand the function and structure of the normal brain but also in diseased brains. For example, as broadly discussed in this thesis, high resolution and multicontrast imaging could support sophisticated quantitative and diffusion microstructure modelling, such as axon diameter modelling and quantitative myelin modelling. Those models (and others) are relevant to understand brain connectivity in normal brain, and how those biological properties are affected in patients with neuro-degenerative diseases like Parkinson [130], Multiple Sclerosis [195] and Epilepsy [229].

From a clinical point of view, on the other hand, time is a valuable parameter given the restriction of scanning human subjects, especially in diseased conditions. In other words, a proper diagnosis (or clinical application) requires an accelerated version of an often lengthy scientific acquisition protocol: acquire enough data to be analysed with sophisticated (mostly data expensive) models, such that the results enable a robust interpretation. Which can be simplified to get as much (good quality) data as possible in the shortest time. Here is where MESMERISED can contribute immensely to this endeavour. In its current state, a full qMRI and dMRI protocol (with included calibration) with MESMERISED can be achieved in 10 to $20 \mathrm{~min}$ (at $1.8 \mathrm{~mm}$ isotropic resolution) at a $7 \mathrm{~T} \mathrm{MR} \mathrm{scanner} \mathrm{(an} \mathrm{lighly} \mathrm{updated}$ version of which has been FDA approved and CE-marked for clinical use), with promising updates for reducing this time even further without compromising data quality, and a potential to extend its implementation to much more commonly used $3 \mathrm{~T}$ clinical scanners.

\subsection{Target group}

Acquiring MR images with these sequences can be interesting to several groups, because the data type and quality required for improving preprocessing methods, refining analysis models and overcoming current software and model limitations will be accessible. For example, high resolution ex vivo data is computer expensive when the available software for image reconstruction, pre-processing and analysis (especially tractography) are used. Then, software developers can improve their software by using this kind of data ${ }^{1}$. MR physicists and Neuroscientists can use this data to study the validity of signal modelling and compare it with information from his-

\footnotetext{
${ }^{1}$ This request was already asked for some software developers during the presentation of the $\mathrm{k}_{\mathrm{T}}-\mathrm{dSTEAM}$ abstract in ISMRM 2018 in Paris, France.
} 
tology, even more if the specimen used is the same. In other words, this can contribute in the aim of in vivo MR histology.

As previously mentioned for the clinical setup, radiologists (or medical physicists) and physicians can experiment with a higher amount of data offered by the MESMERISED sequence. This could help them to estimate how much data and at which resolution would be necessary for a robust analysis or diagnosis. This step is really important because a proper diagnosis can help in the prognosis of the patient.

\subsection{Activity}

All the studies presented in this work are available for the scientific community in the format of poster presentation, power pitch, scientific sessions in international conferences like the International Society of Magnetic Resonance in Medicine (ISMRM) and its corresponding Benelux Chapter (ISMRM Benelux Chapter), the European Society of Magnetic Resonance in Medicine and Biology (ESMRMB) and the Organisation of the Human Brain Mapping (OHBM). Moreover, some of them are published papers with open access (see the references [88] and [87]). Furthermore, the advances in high resolution dMRI of ex vivo human brain tissue have been actively disseminated to the local Maastricht University Medical Centre researchers. The $\mathrm{k}_{\mathrm{T}}$-dSTEAM sequence is a crucial enabling tool in several studies investigating mechanisms and treatments of diseases, such as Epilepsy, Alzheimer's disease, and Parkinson's disease.

The MESMERISED sequence has been patented ${ }^{2}$ to protect the intellectual property inherent to its inventive aspects, and ensure that some benefits from commercialising the technology will flow back to scientific research and academic teaching. As of this writing, the patent has been licensed to one major scanner vendor. Complementary to this, Maastricht University and the inventors commit themselves to make all aspects of MESMERISED available without cost to researchers and clinicians for the purpose of non-commercial research, investigation and teaching. This can allow them to implement it in their respective (research and clinical) centres and expand the advantages of this sequence for research studies as well for clinical studies (as mentioned previously).

\footnotetext{
${ }^{2}$ Inventors A. Roebroeck, B. Poser and F. J. Fritz; CPT and USA patents are pending.
} 


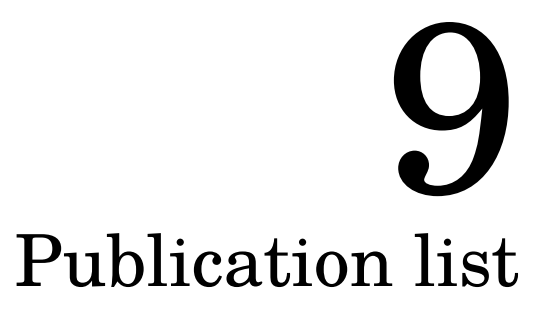


Peer reviewed journal articles

1. Fritz, F.J., Sengupta, S., Harms, R.L., Tse, D.H., Poser, B.A. and Roebroeck, A. (2019). Ultra-high resolution and multi-shell diffusion MRI of intact ex vivo human brains using kT-dSTEAM at 9.4T. NeuroImage Vol. 202: 116087;

doi:10.1016/j.neuroimage.2019.116087

2. Fritz, F.J., Poser, B.A. and Roebroeck, A. (2020). MESMERISED: Super-accelerated 7 T STEAM imaging for quantitative multi-contrast and diffusion MRI. bioRxiv 2020.05.15.098269; doi:10.1101/2020.05.15.098269

3. Fritz, F.J., Poser, B.A. and Roebroeck, A. (under resubmission). MESMERISED: Super-accelerated 7 T STEAM imaging for quantitative multi-contrast and diffusion MRI. NeuroImage.

4. Sengupta, S., Fritz, F.J., Harms, R.L., Hildebrand, S., Tse, D.H., Poser, B.A., Goebel, R. and Roebroeck, A. (2018). High resolution anatomical and quantitative MRI of the entire human occipital lobe ex vivo at 9.4 T. NeuroImage Vol. 168:162-17;

doi:10.1016/j.neuroimage.2017.03.039.

5. Harms, R.L., Fritz, F.J., Schoenmakers, S., Roebroeck, A. (2019). Fast quantification of uncertainty in non-linear diffusion MRI models for artifact detection and more power in group studies. bioRxiv 651547 ; doi:10.1101/651547.

6. Harms, R.L., Fritz, F.J., Tobisch, A., Goebel, R., Roebroeck, A. (2017). Robust and fast nonlinear optimization of diffusion MRI microstructure models. NeuroImage Vol. 155:82-96; doi:10.1016/j.neuroimage.2017.04.064.

\section{Conference proceedings}

1. Fritz, F.J., Poser, B. and Roebroeck, A. MESMERISED: Super-accelerated 7T STEAM imaging for quantitative T1 and diffusion MRI. In Pro- 
ceedings of the 27th International Society for Magnetic Resonance in Medicine, Montreal (2019) 0937. (Oral presentation - Summa cum Laude award).

2. Fritz, F.J., Poser, B. and Roebroeck, A. MESMERISED: Super-accelerated 7T STEAM imaging for quantitative T1 and diffusion MRI. In the the UHF Study Group Business meeting of the proceedings of the 27th International Society for Magnetic Resonance in Medicine, Montreal (2019) (Oral presentation - 1st place award in the category of UHF MRI Technology Development).

3. Fritz, F.J., Poser, B. and Roebroeck, A. MESMERISED: Super-accelerated 7T STEAM imaging for quantitative T1 and diffusion MRI. In Proceedings of the 36th Annual Meeting of the European Society for Magnetic Resonance in Medicine and Biology, Rotterdam (2019) A-1269. (Oral presentation).

4. Fritz, F.J., Poser, B. and Roebroeck, A. MESMERISED: Super-accelerated 7T STEAM imaging for quantitative T1 and diffusion MRI. In Proceedings of the 25th Annual Meeting of the Organization for Human Brain Mapping, Rome (2019) 4357. (Poster - withdrawn).

5. Fritz, F.J., Sengupta, S., Harms, R.L., Poser, B. and Roebroeck, Ultra-high resolution multi-shell dMRI and tractography of the ex vivo human brain using kT-dSTEAM at 9.4T. In Proceedings of the 26th International Society for Magnetic Resonance in Medicine, Paris (2018) 0045. (Oral presentation).

6. Kashyap, S., Fritz, F.J., Harms, R.L., Huber, L., Ivanov, D., Roebroeck, A., Poser, B. and Uludag, K. Effect of optimised coil-combinations on high-resolution laminar fMRI at 9.4T. In Proceedings of the 26th International Society for Magnetic Resonance in Medicine, Paris (2018) 0045. (Oral presentation).

7. Fritz, F.J., Harms, R.L., Sengupta, S., Hildebrand, S. and Roebroeck, A. $400 \mu \mathrm{m}$ dMRI and tractography of early human visual system projections ex vivo using kT-dSTEAM at 9.4T. In Proceedings of the 24th Annual Meeting of the Organization for Human Brain Mapping, Singapore (2018) 1849. (Oral presentation and poster). 
8. Fritz, F.J.. High resolution diffusion MRI and tractography of post mortem brains using kT-dSTEAM at 9.4T. In Proceedings of the 3rd Annual Conference of the IN.NL "Science without frontiers" | Red de Investigadores Chilenos en los Paises Bajos, Groningen (2018) (Oral presentation).

9. Fritz, F.J., Tse, D.H., Sengupta, S., Loderhose, T., Kraaijeveld, B., Caspers, S., Poser, B. and Roebroeck, A. Kt-DSTEAM: High Resolution Diffusion-Weighted Imaging of the Ex Vivo Human Brain Using B1+ Homogenized STEAM at 9.4T. In Proceedings of the 25th International Society for Magnetic Resonance in Medicine, Honolulu (2017) 0173. (Oral presentation - Magna sum Laude award).

10. Fritz, F.J., Tse, D.H., Sengupta, S., Harms, R.L., Loderhose, T., Kraaijeveld, B., Herrler, A., Lataster, A., Caspers, S., Amunts, K., Poser, B.A. and Roebroeck, A. High resolution diffusion MRI and tractography of post mortem human brains using kT-dSTEAM at 9.4T. In Proceedings of the 23rd Annual Meeting of the Organization for Human Brain Mapping, Vancouver (2017) 1567. (Oral presentation and poster).

11. Fritz, F.J., Tse, D.H., Sengupta, S., Loderhose, T., Kraaijeveld, B., Caspers, S., Poser, B. and Roebroeck, A. kT-STEAM: kT-points 3D at $9.4 \mathrm{~T}$ for high resolution whole brain $\mathrm{T} 1$ and $\mathrm{T} 2$ weighted MRI ex vivo. In Proceedings of the 8th International Society for Magnetic Resonance in Medicine Benelux Chapter, Tilburg (2017). (Oral presentation).

12. Sengupta, S., Fritz, F.J., Hellenbrand, R.F., Wiggins, C.R. and Roebroeck, A. A Modular RF Coil Platform for Ex-Vivo Imaging of Brain Slices at 9.4T. In Proceedings of the 25th International Society for Magnetic Resonance in Medicine, Honolulu (2017) 2413. (Poster).

13. (Lagos) Fritz, F.J., Foxley, S., Sengupta, S., Harms, R., Caspers, S., Zilles, K., Tse, D.H., Poser, B., Miller, K. and Roebroeck, A. Whole human brain diffusion MRI at $450 \mu \mathrm{m}$ post mortem with dwSSFP and a specialized 9.4T RF-coil. In Proceedings of the 24th International Society for Magnetic Resonance in Medicine, Singapore (2016) 3379. (Electronic poster). 
14. (Lagos) Fritz, F.J., Foxley, S., Sengupta, S., Harms, R., Caspers, S., Zilles, K., Tse, D.H., Poser, B., Miller, K. and Roebroeck, A. Whole human brain diffusion MRI at $450 \mu \mathrm{m}$ post mortem with dwSSFP and a specialized 9.4T RF-coil. In Proceedings of the 7th International Society for Magnetic Resonance in Medicine Benelux Chapter, Eindhoven (2016). (Poster).

\section{Published patent}

1. Patent code: P2016-054/AB/5634/MESMERISED | AOMB ref: 70321US and US application 16/757,098 "A METHOD AND A MRI APPARATUS FOR OBTAINING IMAGES OF A TARGET VOLUME OF A HUMAN AND/OR ANIMAL SUBJECT USING MAGNETIC RESONANCE IMAGING (MRI)" 



\section{0 \\ Curriculum Vitae}


Francisco, or mostly known as Frank, was born on May 4th, $1990^{1}$, in Santiago of Chile. He finished his secondary education at Colegio San Luis, Antofagasta, Chile, in 2007 (total grade of 6.4/7.0). He studied and graduated in 2015 as a Licentiate in Astronomy at Pontifical Catholic University of Chile (PUC). His thesis, completely unrelated with his degree, focused on the "Parameters optimisation for an Elekta's kV-

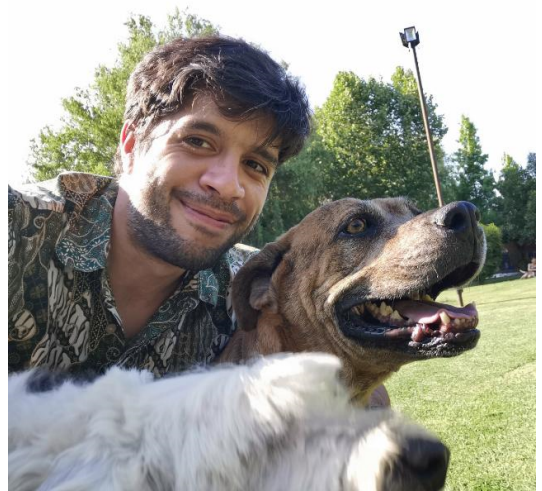
CBCT"2. This allowed him to apply and get accepted in the double Master's degree in Clinical Medical Physics at the same University (PUC) and Heidelberg University, Germany. His Master's Thesis, again completely outside of the aim of the Master's program, was developed during his internship at the German Cancer Research Centre (DKFZ), Heidelberg, under the supervision of Dr. Dorde Komljenovic (DKFZ) and Dr. Ignacio Espinoza (PUC). This work covered the "Calculation of vascular fraction per voxel in experimental tumours using magnetic resonance imaging (MRI)"3. Months later after his Master's defence, he started a $\mathrm{PhD}$ project under the supervision of Prof. Dr. Rainer Goebel and Dr. Alard Roebroeck at Maastricht University. Dr. Benedikt Poser became his supervisor a year later when a proper mentoring on MR sequence programming was required. During the completion of his thesis entitled "MRI methods for diffusion-weighted imaging at Ultra-high field: from ex vivo to in vivo imaging of the human brain", Frank started to work as a Postdoctoral researcher in the group "Quantitative MRI and in vivo histology" at Universitätklinikum Hamburg-Eppendorf under the supervision of Dr. Siawoosh Mohammaddi. Between the academic steps mentioned, he had spent time doing small projects for different research groups and institutions (Live is too short). Jack is still alive (thanks Medicine) and 8 years and 2 months old at the moment this section is being updated before printing.

\footnotetext{
${ }^{1}$ Yes, may the force be with you.

${ }^{2}$ During this period, Jack appeared in his life.

${ }^{3}$ And during this period, his mythical cubic dog mug appeared in his life.
} 


\section{Bibliography}

[1] B. Ades-Aron, J. Veraart, P. Kochunov, S. McGuire, P. Sherman, E. Kellner, D. Novikov, and E. Fieremans. Evaluation of the accuracy and precision of the diffusion parameter estimation with gibbs and noise removal pipeline. NeuroImage, 183:532 - 543, 2018.

[2] M. Aggarwal, J. Y. Zhang, O. Pletnikova, B. Crain, J. Troncoso, and S. Mori. Feasibility of creating a high-resolution $3 \mathrm{~d}$ diffusion tensor imaging based atlas of the human brainstem: A case study at 11.7 t. Neuroimage, 74:117-127, 2013.

[3] M. Aggarwal, D. W. Nauen, J. C. Troncoso, and S. Mori. Probing region-specific microstructure of human cortical areas using high angular and spatial resolution diffusion mri. Neuroimage, 105:198207, 2015.

[4] S. Aja-Fernandez, G. Vegas-Sanchez-Ferrero, and A. Tristan-Vega. Noise estimation in parallel mri: Grappa and sense. Magnetic Resonance Imaging, 32(3):281 - 290, 2014.

[5] D. C. Alexander, P. L. Hubbard, M. G. Hall, E. A. Moore, M. Ptito, G. J. Parker, and T. B. Dyrby. Orientationally invariant indices of axon diameter and density from diffusion mri. Neuroimage, 52(4): 1374-1389, 2010.

[6] D. C. Alexander, T. B. Dyrby, M. Nilsson, and H. Zhang. Imaging 
brain microstructure with diffusion mri: practicality and applications. NMR Biomed, 2017.

[7] T. B. Alexander, D. C.; Dirby. Diffusion imaging with stimulated echoes: signal models and experiment design, 2013.

[8] Y. Assaf and P.J. Basser. Composite hindered and restricted model of diffusion (charmed) $\mathrm{mr}$ imaging of the human brain. NeuroImage, 27(1):48 - 58, 2005.

[9] Y. Assaf and Y. Cohen. Assignment of the water slow-diffusing component in the central nervous system using q-space diffusion mrs: Implications for fiber tract imaging. Magnetic Resonance in Medicine, 43(2):191-199, 2000.

[10] Y. Assaf and O. Pasternak. Diffusion tensor imaging (dti)-based white matter mapping in brain research: a review. J Mol Neurosci, 34(1):51-61, 2008.

[11] Y. Assaf, R. Z. Freidlin, G. K. Rohde, and P. J. Basser. New modeling and experimental framework to characterize hindered and restricted water diffusion in brain white matter. Magnetic Resonance in Medicine, 52(5):965-78, 2004.

[12] Y. Assaf, T. Blumenfeld-Katzir, Y. Yovel, and P. J. Basser. Axcaliber: A method for measuring axon diameter distribution from diffusion mri. Magnetic Resonance in Medicine, 59(6):1347-1354, 2008.

[13] Y. Assaf, D. C. Alexander, D. K. Jones, A. Bizzi, T. E. Behrens, C. A. Clark, Y. Cohen, T. B. Dyrby, P. S. Huppi, T. R. Knoesche, D. Lebihan, G. J. Parker, C. Poupon, Connect consortium, D. Anaby, A. Anwander, L. Bar, D. Barazany, T. Blumenfeld-Katzir, S. De-Santis, D. Duclap, M. Figini, E. Fischi, P. Guevara, P. Hubbard, S. Hofstetter, S. Jbabdi, N. Kunz, F. Lazeyras, A. Lebois, M. G. Liptrot, H. Lundell, J. F. Mangin, D. M. Dominguez, D. Morozov, J. Schreiber, K. Seunarine, S. Nava, C. Poupon, T. Riffert, E. Sasson, B. Schmitt, N. Shemesh, S. N. Sotiropoulos, I. Tavor, H. G. Zhang, and F. L. Zhou. The connect project: Combining macro- and micro-structure. Neuroimage, 80:273-282, 2013. 
[14] J. C. Augustinack, C. Magnain, M. Reuter, A. J. van der Kouwe, D. Boas, and B. Fischl. Mri parcellation of ex vivo medial temporal lobe. Neuroimage, 93 Pt 2:252-259, 2014.

[15] D. Barazany, P. J. Basser, and Y. Assaf. In vivo measurement of axon diameter distribution in the corpus callosum of rat brain. Brain, 132 (Pt 5):1210-1220, 2009.

[16] A. Barnett, E. Hutchinson, O. Irfanoglu, and C. Pierpaoli. Higher order correction of eddy current distortion in diffusion weighted echo planar images. In ISMRM 23rd Annual Meeting \& Exhibition, 2014.

[17] P. Basser and C. Pierpaoli. Microstructural and physiological features of tissues elucidated by quantitative-diffusion-tensor mri. Journal of Magnetic Resonance, 213(2):560 - 570, 2011.

[18] P. J. Basser, J. Mattiello, and D. Le Bihan. Estimation of the effective self-diffusion tensor from the nmr spin echo. Journal of Magnetic Resonance, Series B, 103(3):247 - 254, 1994.

[19] P. J. Basser, J. Mattiello, and D. LeBihan. Mr diffusion tensor spectroscopy and imaging. Biophys J, 66(1):259-67, 1994.

[20] P. J. Basser, S. Pajevic, C. Pierpaoli, J. Duda, and A. Aldroubi. In vivo fiber tractography using dt-mri data. Magnetic Resonance in Medicine, 44(4):625-632, 2000.

[21] M. Bastiani, N.J. Shah, R. Goebel, and A. Roebroeck. Human cortical connectome reconstruction from diffusion weighted mri: The effect of tractography algorithm. NeuroImage, 62(3):1732 - 1749, 2012 .

[22] M. Bastiani, A.-M. Oros-Peusquens, A. Seehaus, D. Brenner, K. Möllenhoff, A. Celik, J. Felder, H. Bratzke, N. J. Shah, R. Galuske, R. Goebel, and A. Roebroeck. Automatic segmentation of human cortical layer-complexes and architectural areas using ex vivo diffusion mri and its validation. Frontiers in Neuroscience, 10:487, 2016.

[23] C. Beaulieu. The basis of anisotropic water diffusion in the nervous system - a technical review. NMR Biomed, 15(7-8):435-55, 2002. 
[24] S. M. Becker, K. Tabelow, S. Mohammadi, N. Weiskopf, and J. Polzehl. Adaptive smoothing of multi-shell diffusion weighted magnetic resonance data by mspoas. Neuroimage, 95:90-105, 2014.

[25] T. E. Behrens, H. J. Berg, S. Jbabdi, M. F. Rushworth, and M. W. Woolrich. Probabilistic diffusion tractography with multiple fibre orientations: What can we gain? Neuroimage, 34(1):144-55, 2007.

[26] T.E.J. Behrens, M.W. Woolrich, M. Jenkinson, H. Johansen-Berg, R.G. Nunes, S. Clare, P.M. Matthews, J.M. Brady, and S.M. Smith. Characterization and propagation of uncertainty in diffusionweighted $\mathrm{mr}$ imaging. Magnetic Resonance in Medicine, 50(5):1077$1088,2003$.

[27] Matt A. Bernstein, Kevin F. King, and Xiaohong Joe Zhou. CHAPTER 9 - MOTION-SENSITIZING GRADIENTS, pages 274-291. Burlington, 2004.

[28] A. I. Blazejewska, H. Bhat, L. L. Wald, and J. R. Polimeni. Reduction of across-run variability of temporal snr in accelerated epi timeseries data through fleet-based robust autocalibration. Neuroimage, 152:348-359, 2017.

[29] F. Bloch. Nuclear induction. Phys. Rev., 70:460-474, 1946.

[30] F. Bloch, W. W. Hansen, and M. Packard. Nuclear induction. Phys. Rev., 69:127-127, 1946.

[31] K. Block and J. Frahm. Spiral imaging: A critical appraisal. Journal of Magnetic Resonance Imaging, 21(6):657-668, 2005.

[32] I. G. Bloomfield, I. H. Johnston, and L. E. Bilston. Effects of proteins, blood cells and glucose on the viscosity of cerebrospinal fluid. Pediatric Neurosurgery, 28:246-251, 1998.

[33] P. A. Bottomley, T. H. Foster, R. E. Argersinger, and L. M. Pfeifer. A review of normal tissue hydrogen nmr relaxation times and relaxation mechanisms from 1-100 mhz: Dependence on tissue type, $\mathrm{nmr}$ frequency, temperature, species, excision, and age. Medical Physics, 11(4):425-448, 1984. 
[34] F. A. Breuer, M. Blaimer, M. F. Mueller, N. Seiberlich, R. M. Heidemann, M. A. Griswold, and P. M. Jakob. Controlled aliasing in volumetric parallel imaging (2d caipirinha). Magnetic Resonance in Medicine, 55(3):549-56, 2006.

[35] F. A. Breuer, S. A.R. Kannengiesser, M. Blaimer, N. Seiberlich, P. M. Jakob, and M. A. Griswold. General formulation for quantitative gfactor calculation in grappa reconstructions. Magnetic Resonance in Medicine, 62(3):739-746, 2009.

[36] R.G. Brewer and E.L. Hahn. Atomic memory. Scientific American, 251:50-57, 1984.

[37] H. Bridge and S. Clare. High-resolution mri: <i>in vivo</i> histology? Philosophical Transactions of the Royal Society B: Biological Sciences, 361(1465):137-146, 2006.

[38] Korbinian Brodmann. Brodmann's: Localisation in the cerebral cortex. Springer Science \& Business Media, 2007.

[39] L. M. Burcaw, E. Fieremans, and D. S. Novikov. Mesoscopic structure of neuronal tracts from time-dependent diffusion. Neuroimage, 114:18-37, 2015.

[40] Deborah Burstein. Stimulated echoes: Description, applications, practical hints. Concepts in Magnetic Resonance, 8(4):269-278, 1996.

[41] R. B. Buxton. The diffusion sensitivity of fast steady-state free precession imaging. Magnetic Resonance in Medicine, 29(2):235-243, 1993.

[42] E. Calabrese, P. Hickey, C. Hulette, J. Zhang, B. Parente, S. P. Lad, and G. A. Johnson. Postmortem diffusion mri of the human brainstem and thalamus for deep brain stimulator electrode localization. Hum Brain Mapp, 36(8):3167-3178, 2015.

[43] M. F. Callaghan, G. Helms, A. Lutti, S. Mohammadi, and N. Weiskopf. A general linear relaxometry model of $\mathrm{r} 1 \mathrm{using}$ imaging data. Magnetic resonance imaging, 73(3):1309-14, 2015. 
[44] P.T. Callaghan. Principles of Nuclear Magnetic Resonance Microscopy. Oxford science publications. Clarendon Press, 1993.

[45] P.T. Callaghan. Nmr imaging, nmr diffraction and applications of pulsed gradient spin echoes in porous media. Magnetic resonance imaging, 14(7-8):701-709, 1996.

[46] J. Campbell, I. Leppert, S. Narayanan, M. Boudreau, T. Duval, J. Cohen-Adad, G.B. Pike, and N. Stikov. Promise and pitfalls of g-ratio estimation with mri. NeuroImage, 182:80 - 96, 2018.

[47] S. F. Cauley, J. R. Polimeni, H. Bhat, L. L. Wald, and K. Setsompop. Interslice leakage artifact reduction technique for simultaneous multislice acquisitions. Magnetic Resonance in Medicine, 72(1): 93-102, 2014.

[48] M. Cercignani and S. Bouyagoub. Brain microstructure by multimodal mri: Is the whole greater than the sum of its parts? NeuroImage, 182:117 - 127, 2018.

[49] M. Cercignani, N. Dowell, and P. Tofts. Series in Medical Physics and Biomedical Engineering. Boca Raton: CRC Press, 2nd edition edition, 2018.

[50] G. B. Chavhan, P. S. Babyn, B. Thomas, M. M. Shroff, and E. M. Haacke. Principles, techniques, and applications of $\mathrm{t} 2 *$-based $\mathrm{mr}$ imaging and its special applications. RadioGraphics, 29(5):1433$1449,2009$.

[51] S. Chung, D. Kim, E. Breton, and L. Axel. Rapid b1+ mapping using a preconditioning rf pulse with turboflash readout. Magnetic Resonance in Medicine, 64(2):439-446, 2010.

[52] C.A. Clark, M. Hedehus, and M.E. Moseley. In vivo mapping of the fast and slow diffusion tensors in human brain. Magnetic Resonance in Medicine, 47(4):623-628, 2002.

[53] M. A. Cloos, N. Boulant, M. Luong, G. Ferrand, E. Giacomini, D. Le Bihan, and A. Amadon. kt -points: short three-dimensional 
tailored rf pulses for flip-angle homogenization over an extended volume. Magnetic Resonance in Medicine, 67(1):72-80, 2012.

[54] L. Cordero-Grande, D. Christiaens, J. Hutter, A. N. Price, and J. V. Hajnal. Complex diffusion-weighted image estimation via matrix recovery under general noise models. Neuroimage, 200:391-404, 2019.

[55] D G Cory and A N Garroway. Measurement of translational displacement probabilities by nmr: an indicator of compartmentation. Magnetic Resonance in Medicine, 14(3):435-44, 1990.

[56] J. Crank and E.P.J. Crank. The Mathematics of Diffusion. Oxford science publications. Clarendon Press, 1979.

[57] R. Cusack and N. Papadakis. New robust 3-d phase unwrapping algorithms: application to magnetic field mapping and undistorting echoplanar images. NeuroImage, 16(3 Pt 1):754-764, 2002.

[58] A. Daducci, A. Dal Palu, A. Lemkaddem, and A. Thiran. Commit: Convex optimization modeling for microstructure informed tractography. IEEE Transactions on Medical Imaging, 34(1):246-257, 2015.

[59] H. D'Arceuil and A. de Crespigny. The effects of brain tissue decomposition on diffusion tensor imaging and tractography. Neuroimage, 36(1):64-68, 2007.

[60] H. E. D'Arceuil, S. Westmoreland, and A. J. de Crespigny. An approach to high resolution diffusion tensor imaging in fixed primate brain. Neuroimage, 35(2):553-565, 2007.

[61] S. De Santis, Y. Assaf, C. J. Evans, and D. K. Jones. Improved precision in charmed assessment of white matter through sampling scheme optimization and model parsimony testing. Magnetic Resonance in Medicine, 71(2):661-671, 2014.

[62] S. De Santis, M. Drakesmith, S. Bells, Y. Assaf, and D. K. Jones. Why diffusion tensor mri does well only some of the time: variance and covariance of white matter tissue microstructure attributes in the living human brain. Neuroimage, 89:35-44, 2014. 
[63] S. De Santis, Y. Assaf, B. Jeurissen, D. K. Jones, and A. Roebroeck. T1 relaxometry of crossing fibres in the human brain. Neuroimage, 141:133-142, 2016.

[64] S. De Santis, D. Barazany, D. K. Jones, and Y. Assaf. Resolving relaxometry and diffusion properties within the same voxel in the presence of crossing fibres by combining inversion recovery and diffusionweighted acquisitions. Magnetic Resonance in Medicine, 75(1):372380, 2016.

[65] S. De Santis, D. K. Jones, and A. Roebroeck. Including diffusion time dependence in the extra-axonal space improves in vivo estimates of axonal diameter and density in human white matter. Neuroimage, 130:91-103, 2016.

[66] S. De Santis, P. Bach, L. Perez-Cervera, A. Cosa-Linan, G. Weil, S. Vollstädt-Klein, D. Hermann, F. Kiefer, P. Kirsch, R. Ciccocioppo, W. H. Sommer, and S. Canals. Microstructural white matter alterations in men with alcohol use disorder and rats with excessive alcohol consumption during early abstinence. JAMA Psychiatry, 76(7): 749-758, 2019.

[67] F. Dell'Acqua, I. Bodi, D. Slater, M. Catani, and M. Modo. Mr diffusion histology and micro-tractography reveal mesoscale features of the human cerebellum. Cerebellum, 12(6):923-931, 2013.

[68] M. Does. Inferring brain tissue composition and microstructure via mr relaxometry. NeuroImage, 182:136 - 148, 2018.

[69] T. B. Dyrby, W. F. Baare, D. C. Alexander, J. Jelsing, E. Garde, and L. V. Sogaard. An ex vivo imaging pipeline for producing highquality and high-resolution diffusion-weighted imaging datasets. Hum Brain Mapp, 32(4):544-563, 2011.

[70] T. B. Dyrby, L. V. Sogaard, M. G. Hall, M. Ptito, and D. C. Alexander. Contrast and stability of the axon diameter index from microstructure imaging with diffusion mri. Magnetic Resonance in Medicine, 2012 .

[71] T. B. Dyrby, G. M. Innocenti, M. Bech, and H. Lundell. Validation 
strategies for the interpretation of microstructure imaging using diffusion mri. Neuroimage, 2018.

[72] F. Eggenschwiler, K. R. O'Brien, R. Gruetter, and J. P. Marques. Improving $\mathrm{t} 2$-weighted imaging at high field through the use of $\mathrm{kt}$ -points. Magnetic Resonance in Medicine, 71(4):1478-1488, 2014.

[73] F. Eggenschwiler, K. R. O'Brien, D. Gallichan, R. Gruetter, and J. P. Marques. 3d t 2-weighted imaging at $7 \mathrm{t}$ using dynamic kt-points on single-transmit mri systems. MAGMA, 29(3):347-358, 2016.

[74] P. Ehses, J. Bause, G. Shajan, and K. Scheffler. Efficient generation of $\mathrm{t} 2 *$-weighted contrast by interslice echo-shifting for human functional and anatomical imaging at 9.4 tesla. Magnetic Resonance in Medicine, 74(6):1698-704, 2015.

[75] C. Eichner, L. L. Wald, and K. Setsompop. A low power radiofrequency pulse for simultaneous multislice excitation and refocusing. Magnetic Resonance in Medicine, 72(4):949-58, 2014.

[76] A. Einstein. Investigations on the Theory of the Brownian Movement. Dover Books on Physics Series. Dover Publications, 1956.

[77] A D Elster. Gradient-echo mr imaging: techniques and acronyms. Radiology, 186(1):1-8, 1993.

[78] Q. Fan, A. Nummenmaa, J. R. Polimeni, T. Witzel, S. Y. Huang, V. J. Wedeen, B. R. Rosen, and L. L. Wald. High b-value and high resolution integrated diffusion (hibrid) imaging. Neuroimage, 150:162176, 2017.

[79] G. M. Fatterpekar, T. P. Naidich, B. N. Delman, J. G. Aguinaldo, S. H. Gultekin, C. C. Sherwood, P. R. Hof, B. P. Drayer, and Z. A. Fayad. Cytoarchitecture of the human cerebral cortex: $\mathrm{Mr}$ microscopy of excised specimens at 9.4 tesla. AJNR Am J Neuroradiol, 23(8):1313-1321, 2002.

[80] D. A. Feinberg, S. Moeller, S. M. Smith, E. Auerbach, S. Ramanna, M. Gunther, M. F. Glasser, K. L. Miller, K. Ugurbil, and E. Yacoub. 
Multiplexed echo planar imaging for sub-second whole brain fmri and fast diffusion imaging. PLoS One, 5(12):e15710, 2010.

[81] Adolf Fick. Ueber diffusion. Annalen der Physik, 170(1):59-86, 1855.

[82] E. Fieremans, A. Benitez, J. H. Jensen, M. F. Falangola, A. Tabesh, R. L. Deardorff, M. V. Spampinato, J. S. Babb, D. S. Novikov, S. H. Ferris, and J. A. Helpern. Novel white matter tract integrity metrics sensitive to alzheimer disease progression. AJNR Am J Neuroradiol, 34(11):2105-12, 2013.

[83] E. Fieremans, L.M. Burcaw, H.-H. Lee, G. Lemberskiy, J. Veraart, and D.S. Novikov. In vivo observation and biophysical interpretation of time-dependent diffusion in human white matter. NeuroImage, 129:414 - 427, 2016.

[84] S. Foxley, S. Jbabdi, S. Clare, W. Lam, O. Ansorge, G. Douaud, and K. Miller. Improving diffusion-weighted imaging of post-mortem human brains: Ssfp at 7 t. Neuroimage, 102 Pt 2:579-589, 2014.

[85] J. Frahm, K. D. Merboldt, W. Hänicke, and A. Haase. Stimulated echo imaging. Journal of Magnetic Resonance (1969), 64(1):81-93, 1985 .

[86] F. Fritz, R. Harms, S. Sengupta, S. Hildebrand, and A. Roebroeck. $400 \mu \mathrm{m}$ dmri and tractography of early human visual system projections ex vivo using kt-dsteam at 9.4 t. In Proceedings of the 2018 Organization for Human Brain Mapping annual meeting, 2018.

[87] F. J. Fritz, B. A. Poser, and A. Roebroeck. Mesmerised: Superaccelerated $7 \mathrm{t}$ steam imaging for quantitative multi-contrast and diffusion mri. bioRxiv, 2020.

[88] F.J. Fritz, S. Sengupta, R.L. Harms, D.H. Tse, B.A. Poser, and A. Roebroeck. Ultra-high resolution and multi-shell diffusion mri of intact ex vivo human brains using kt-dsteam at 9.4t. NeuroImage, 202:116087, 2019.

[89] M. Fukunaga, T. Q. Li, P. van Gelderen, J. A. de Zwart, K. Shmueli, B. Yao, J. Lee, D. Maric, M. A. Aronova, G. Zhang, R. D. Leapman, 
J. F. Schenck, H. Merkle, and J. H. Duyn. Layer-specific variation of iron content in cerebral cortex as a source of mri contrast. Proc Natl Acad Sci U S A, 107(8):3834-3839, 2010.

[90] A. Gibson, A. M. Peters, and R. Bowtell. Echo-shifted multislice epi for high-speed fmri. Magnetic Resonance Imaging, 24(4):433$42,2006$.

[91] M. Glasser, S. Sotiropoulos, J. Wilson, T. Coalson, B. Fischl, J. Andersson, J. Xu, S. Jbabdi, M. Webster, J. Polimeni, D. Van Essen, and M. Jenkinson. The minimal preprocessing pipelines for the human connectome project. NeuroImage, 80:105 - 124, 2013.

[92] P. M. Glover, R. W. Bowtell, G. D. Brown, and P. Mansfield. A microscope slide probe for high resolution imaging at 11.7 tesla. Magnetic resonance in medicine, 31(4):423-428, 1994.

[93] M. Goldman. Formal theory of spin-lattice relaxation. Journal of Magnetic Resonance, 149(2):160 - 187, 2001.

[94] M. A. Griswold, P. M. Jakob, R. M. Heidemann, M. Nittka, V. Jellus, J. Wang, B. Kiefer, and A. Haase. Generalized autocalibrating partially parallel acquisitions (grappa). Magnetic Resonance in Medicine, 47(6):1202-10, 2002.

[95] H. Gudbjartsson and S. Patz. The rician distribution of noisy mri data. Magnetic Resonance in Medicine, 34(6):910-914, 1995.

[96] A.G. Guggisberg, P.J. Koch, F.C. Hummel, and C.M. Buetefisch. Brain networks and their relevance for stroke rehabilitation. Clinical Neurophysiology, 130(7):1098 - 1124, 2019.

[97] D. N. Guilfoyle, J. A. Helpern, and K. O. Lim. Diffusion tensor imaging in fixed brain tissue at 7.0 t. NMR Biomed, 16(2):77-81, 2003.

[98] M.L. Gyngell. The application of steady-state free precession in rapid 2dft nmr imaging: Fast and ce-fast sequences. Magnetic Resonance Imaging, 6(4):415 - 419, 1988.

[99] A. Haase, J. Frahm, D. Matthaei, W. Hanicke, and K.D. Merboldt. 
Flash imaging. rapid nmr imaging using low flip-angle pulses. Journal of Magnetic Resonance, 67(2):258 - 266, 1986.

[100] E. L. Hahn. Spin echoes. Physical Review, 80:580-594, 1950.

[101] R. L. Harms, F. J. Fritz, A. Tobisch, R. Goebel, and A. Roebroeck. Robust and fast nonlinear optimization of diffusion mri microstructure models. Neuroimage, 155:82-96, 2017.

[102] R.L. Harms, F.J. Fritz, S. Schoenmakers, and A. Roebroeck. Fast quantification of uncertainty in non-linear diffusion mri models for artifact detection and more power in group studies. bioRxiv, page 651547, 2019.

[103] S. Herculano-Houzel. The human brain in numbers: a linearly scaled-up primate brain. Frontiers in Human Neuroscience, 3:31, 2009 .

[104] M. Holz, S. R. Heil, and A. Sacco. Temperature-dependent selfdiffusion coefficients of water and six selected molecular liquids for calibration in accurate $1 \mathrm{~h} \mathrm{nmr}$ pfg measurements. Phys. Chem. Chem. Phys., 2:4740-4742, 2000.

[105] J. Hutter, P. J. Slator, D. Christiaens, Rpag Teixeira, T. Roberts, L. Jackson, A. N. Price, S. Malik, and J. V. Hajnal. Integrated and efficient diffusion-relaxometry using zebra. Sci Rep, 8(1):15138, 2018.

[106] M. Irfanoglu, P. Modi, A. Nayak, A. Knutsen, J. Sarlls, and C. Pierpaoli. Dr-buddi: Diffeomorphic registration for blip up-down diffusion imaging. In Medical Image Computing and Computer-Assisted Intervention - MICCAI 2014, 2014.

[107] D. Ivanov, A. Schafer, M. N. Streicher, R. M. Heidemann, R. Trampel, and R. Turner. A simple low-sar technique for chemical-shift selection with high-field spin-echo imaging. Magnetic Resonance in Medicine, 64(2):319-26, 2010.

[108] I. O. Jelescu and M. D. Budde. Design and validation of diffusion mri models of white matter. Frontiers in Physics, 5:61, 2017. 
[109] I. O. Jelescu, J. Veraart, V. Adisetiyo, S. S. Milla, D. S. Novikov, and E. Fieremans. One diffusion acquisition and different white matter models: how does microstructure change in human early development based on wmti and noddi? Neuroimage, 107:242-256, 2015.

[110] M. Jenkinson, C. F. Beckmann, T. E. J. Behrens, M. W. Woolrich, and S. M. Smith. Fsl. NeuroImage, 62(2):782 - 790, 2012.

[111] J. H. Jensen, J. A. Helpern, A. Ramani, H. Lu, and K. Kaczynski. Diffusional kurtosis imaging: The quantification of non-gaussian water diffusion by means of magnetic resonance imaging. Magnetic Resonance in Medicine, 53(6):1432-1440, 2005.

[112] S. N. Jespersen, J. L. Olesen, B. Hansen, and N. Shemesh. Diffusion time dependence of microstructural parameters in fixed spinal cord. Neuroimage, 182:329-342, 2018.

[113] B. Jeurissen, M. Descoteaux, S. Mori, and A. Leemans. Diffusion mri fiber tractography of the brain. NMR in Biomedicine, 32(4):e3785, 2019 .

[114] D. K. Jones. The effect of gradient sampling schemes on measures derived from diffusion tensor mri: a monte carlo study. Magnetic Resonance in Medicine, 51(4):807-15, 2004.

[115] D. K. Jones and P. J. Basser. "squashing peanuts and smashing pumpkins": How noise distorts diffusion-weighted mr data. Magnetic Resonance in Medicine, 52(5):979-993, 2004.

[116] D. K. Jones and M. Cercignani. Twenty-five pitfalls in the analysis of diffusion mri data. NMR in Biomedicine, 23(7):803-820, 2010.

[117] D.K. Jones, T.R. Knoesche, and R. Turner. White matter integrity, fiber count, and other fallacies: The do's and don'ts of diffusion mri. NeuroImage, 73:239 - 254, 2013.

[118] D.K. Jones, D.C. Alexander, R. Bowtell, M. Cercignani, F. Dell'Acqua, D.J. McHugh, K.L. Miller, M. Palombo, G.J.M. Parker, U.S. Rudrapatna, and C.M.W. Tax. Microstructural imaging of the human brain with a 'super-scanner': 10 key advantages 
of ultra-strong gradients for diffusion mri. NeuroImage, 182:8 - 38, 2018.

[119] P. Kellman and E. R. McVeigh. Image reconstruction in snr units: a general method for snr measurement. Magnetic Resonance in Medicine, 54(6):1439-1447, 2005.

[120] B. Klein, W. Kuschinsky, H. Schrock, and F. Vetterlein. Interdependency of local capillary density, blood flow, and metabolism in rat brains. American Journal of Physiology-Heart and Circulatory Physiology, 251(6):H1333-H1340, 1986.

[121] M. Kleinnijenhuis, V. Zerbi, B. Kusters, C. H. Slump, M. Barth, and A. M. van Cappellen van Walsum. Layer-specific diffusion weighted imaging in human primary visual cortex in vitro. Cortex, 49(9): 2569-2582, 2013.

[122] M. Kleinnijenhuis, J. Mollink, W. W. Lam, P. Kinchesh, A. A. Khrapitchev, S. C. Smart, S. Jbabdi, and K. L. Miller. Choice of reference measurements affects quantification of long diffusion time behaviour using stimulated echoes. Magnetic Resonance in Medicine, 79(2):952-959, 2018.

[123] Natalya Korogod, Carl CH Petersen, and Graham W Knott. Ultrastructural analysis of adult mouse neocortex comparing aldehyde perfusion with cryo fixation. eLife, 4:e05793, 2015.

[124] J. Kärger and W. Heink. The propagator representation of molecular transport in microporous crystallites. Journal of Magnetic Resonance (1969), 51(1):1 - 7, 1983.

[125] M. E. Ladd, P. Bachert, M. Meyerspeer, E. Moser, A. M. Nagel, D.G. Norris, S. Schmitter, O. Speck, S. Straub, and M. Zaiss. Pros and cons of ultra-high-field mri/mrs for human application. Progress in Nuclear Magnetic Resonance Spectroscopy, 109:1 - 50, 2018.

[126] D. LeBihan. Ivim method measures diffusion and perfusion. Diagn Imaging (San Franc), 12(6):133-136, 1990. 
[127] D. LeBihan. Molecular diffusion, tissue microdynamics and microstructure. NMR in Biomedicine, 8(7):375-386, 1995.

[128] H.-H. Lee, E. Fieremans, and D.S. Novikov. What dominates the time dependence of diffusion transverse to axons: Intra- or extraaxonal water? NeuroImage, 182:500 - 510, 2018.

[129] T. B. Leergaard, N. S. White, A. de Crespigny, I. Bolstad, H. D'Arceuil, J. G. Bjaalie, and A. M. Dale. Quantitative histological validation of diffusion mri fiber orientation distributions in the rat brain. PLoS One, 5(1):e8595, 2010.

[130] S. Lehericy, D. Vaillancourt, K. Seppi, O. Monchi, I. Rektorova, A. Antonini, M. McKeown, M. Masellis, D. Berg, J. Rowe, S. Lewis, C. Williams-Gra, A. Tessitore, $H$. on behalf of the International Parkinson Siebner, and Movement Disorder Society (IPMDS)Neuroimaging Study Group. The role of high-field magnetic resonance imaging in parkinsonian disorders: Pushing the boundaries forward. Movement Disorders, 32(4):510-525, 2017.

[131] C. W. Leuze, A. Anwander, P. L. Bazin, B. Dhital, C. Stuber, K. Reimann, S. Geyer, and R. Turner. Layer-specific intracortical connectivity revealed with diffusion mri. Cereb Cortex, 24(2):328$339,2014$.

[132] K. Li, H. Li, X. Y. Zhang, A. M. Stokes, X. Jiang, H. Kang, C. C. Quarles, Z. Zu, D. F. Gochberg, J. C. Gore, and J. Xu. Influence of water compartmentation and heterogeneous relaxation on quantitative magnetization transfer imaging in rodent brain tumors. Magnetic Resonance in Medicine, 76(2):635-44, 2016.

[133] Z.P. Liang and P.C. Lauterbur. Principles of Magnetic Resonance Imaging: A Signal Processing Perspective. 1999.

[134] D. Liewald, R. Miller, N. Logothetis, H.J. Wagner, and A. Schuez. Distribution of axon diameters in cortical white matter: an electronmicroscopic study on three human brains and a macaque. Biological Cybernetics, 108(5):541-557, 2014.

[135] S. Ljunggren. A simple graphical representation of fourier-based 
imaging methods. Journal of Magnetic Resonance (1969), 54(2):338 $-343,1983$.

[136] L. Lu, B. Erokwu, G. Lee, V. Gulani, M. A. Griswold, K. M. Dell, and C. A. Flask. Diffusion-prepared fast imaging with steady-state free precession (dp-fisp): a rapid diffusion mri technique at $7 \mathrm{t}$. Magnetic Resonance in Medicine, 68(3):868-73, 2012.

[137] H. Lundell, D. C. Alexander, and T. B. Dyrby. High angular resolution diffusion imaging with stimulated echoes: compensation and correction in experiment design and analysis. NMR Biomed, 27(8): 918-925, 2014.

[138] A. Lutti, J. Stadler, O. Josephs, C. Windischberger, O. Speck, J. Bernarding, C. Hutton, and N. Weiskopf. Robust and fast whole brain mapping of the rf transmit field b1 at 7t. PLoS One, 7(3): e32379, 2012.

[139] A. Lutti, F. Dick, M. I. Sereno, and N. Weiskopf. Using highresolution quantitative mapping of $\mathrm{r} 1$ as an index of cortical myelination. Neuroimage, 93 Pt 2:176-188, 2014.

[140] K.V. Mardia and P.E. Jupp. Directional statistics, volume 494. John Wiley \& Sons, 2009.

[141] M. Marin-Padilla. The human brain intracerebral microvascular system: development and structure. Frontiers in Neuroanatomy, 6: 38,2012 .

[142] J. P. Marques, T. Kober, G. Krueger, W. van der Zwaag, P. F. Van de Moortele, and R. Gruetter. Mp2rage, a self bias-field corrected sequence for improved segmentation and t1-mapping at high field. Neuroimage, 49(2):1271-81, 2010.

[143] J. A. McNab and K. L. Miller. Sensitivity of diffusion weighted steady state free precession to anisotropic diffusion. Magnetic Resonance in Medicine, 60(2):405-413, 2008.

[144] J. A. McNab, S. Jbabdi, S. C. Deoni, G. Douaud, T. E. Behrens, and K. L. Miller. High resolution diffusion-weighted imaging in fixed 
human brain using diffusion-weighted steady state free precession. Neuroimage, 46(3):775-785, 2009.

[145] K. D. Merboldt, W. Hanicke, and J. Frahm. Diffusion imaging using stimulated echoes. Magnetic Resonance in Medicine, 19(2):233-239, 1991.

[146] K. L. Miller, C. J. Stagg, G. Douaud, S. Jbabdi, S. M. Smith, T. E. Behrens, M. Jenkinson, S. A. Chance, M. M. Esiri, N. L. Voets, N. Jenkinson, T. Z. Aziz, M. R. Turner, H. Johansen-Berg, and J. A. McNab. Diffusion imaging of whole, post-mortem human brains on a clinical mri scanner. Neuroimage, 57(1):167-181, 2011.

[147] K. L. Miller, J. A. McNab, S. Jbabdi, and G. Douaud. Diffusion tractography of post-mortem human brains: optimization and comparison of spin echo and steady-state free precession techniques. Neuroimage, 59(3):2284-2297, 2012.

[148] S. Moeller, E. Yacoub, C. A. Olman, E. Auerbach, J. Strupp, N. Harel, and K. Ugurbil. Multiband multislice ge-epi at 7 tesla, with 16fold acceleration using partial parallel imaging with application to high spatial and temporal whole-brain fmri. Magnetic Resonance in Medicine, 63(5):1144-53, 2010.

[149] S. Mohammadi, H. Möller, H. Kugel, D. Müller, and M. Deppe. Correcting eddy current and motion effects by affine whole-brain registrations: Evaluation of three-dimensional distortions and comparison with slicewise correction. Magnetic Resonance in Medicine, 64 (4):1047-1056, 2010.

[150] J. Mollink, M. Kleinnijenhuis, van Cappellen van Walsum A-M., S.N. Sotiropoulos, M. Cottaar, C. Mirfin, Heinrich M. P., M. Jenkinson, M. Pallebage-Gamarallage, O. Ansorge, S. Jbabdi, and K. L. Miller. Evaluating fibre orientation dispersion in white matter: Comparison of diffusion mri, histology and polarized light imaging. NeuroImage, 157:561 - 574, 2017.

[151] R. J. Nabuurs, I. Hegeman, R. Natte, S. G. van Duinen, M. A. van Buchem, L. van der Weerd, and A. G. Webb. High-field mri of single histological slices using an inductively coupled, self-resonant mi- 
crocoil: application to ex vivo samples of patients with alzheimer's disease. NMR in biomedicine, 24(4):351-357, 2011.

[152] K. Nehrke and P. Bornert. Dream-a novel approach for robust, ultrafast, multislice b(1) mapping. Magnetic Resonance in Medicine, 68(5):1517-1526, 2012.

[153] T. Niendorf, R.M. Dijkhuizen, D.G. Norris, M. van Lookeren Campagne, and K. Nicolay. Biexponential diffusion attenuation in various states of brain tissue: implications for diffusion-weighted imaging. Magnetic Resonance in Medicine, 36(6):847-857, 1996.

[154] M. Nilsson, D. van Westen, F. Stahlberg, P. C. Sundgren, and J. Latt. The role of tissue microstructure and water exchange in biophysical modelling of diffusion in white matter. MAGMA, 26(4):345-70, 2013.

[155] D. G. Norris, P. J. Koopmans, R. Boyacioglu, and M. Barth. Power independent of number of slices (pins) radiofrequency pulses for lowpower simultaneous multislice excitation. Magnetic Resonance in Medicine, 66(5):1234-40, 2011.

[156] D. G. Norris, R. Boyacioglu, J. Schulz, M. Barth, and P. J. Koopmans. Application of pins radiofrequency pulses to reduce power deposition in rare/turbo spin echo imaging of the human head. Magnetic Resonance in Medicine, 71(1):44-9, 2014.

[157] D. S. Novikov, J. H. Jensen, J. A. Helpern, and E. Fieremans. Revealing mesoscopic structural universality with diffusion. Proc Natl Acad Sci U S A, 111(14):5088-93, 2014.

[158] D. S. Novikov, V. G. Kiselev, and S. N. Jespersen. On modeling. Magnetic Resonance in Medicine, 79(6):3172-3193, 2018.

[159] D. S. Novikov, E. Fieremans, S. N. Jespersen, and V. G. Kiselev. Quantifying brain microstructure with diffusion mri: Theory and parameter estimation. NMR in Biomedicine, 32(4):e3998, 2019.

[160] K. R. O’Brien, T. Kober, P. Hagmann, P. Maeder, J. Marques, F. Lazeyras, G. Krueger, and A. Roche. Robust t1-weighted struc- 
tural brain imaging and morphometry at $7 \mathrm{t}$ using mp2rage. PLoS One, 9(6):e99676, 2014.

[161] E. Panagiotaki, T. Schneider, B. Siow, M.G. Hall, M.F. Lythgoe, and D.C. Alexander. Compartment models of the diffusion $\mathrm{mr}$ signal in brain white matter: A taxonomy and comparison. NeuroImage, 59 (3):2241 - 2254, 2012.

[162] T. Paus. Imaging microstructure in the living human brain: A viewpoint. NeuroImage, 182:3 - 7, 2018. Microstructural Imaging.

[163] A. Pfefferbaum, E. V. Sullivan, E. Adalsteinsson, T. Garrick, and C. Harper. Postmortem mr imaging of formalin-fixed human brain. Neuroimage, 21(4):1585-1595, 2004.

[164] J. Pfeuffer, P. F. Van de Moortele, K. Ugurbil, X. Hu, and G. H. Glover. Correction of physiologically induced global off-resonance effects in dynamic echo-planar and spiral functional imaging. Magnetic Resonance in Medicine, 47(2):344-353, 2002.

[165] C. Pierpaoli, P. Jezzard, P. J. Basser, A. Barnett, and G. Di Chiro. Diffusion tensor mr imaging of the human brain. Radiology, 201(3): 637-48, 1996.

[166] B. A. Poser and D. G. Norris. Investigating the benefits of multi-echo epi for fmri at 7 t. Neuroimage, 45(4):1162-72, 2009.

[167] B. A. Poser, R. J. Anderson, B. Guerin, K. Setsompop, W. Deng, A. Mareyam, P. Serano, L. L. Wald, and V. A. Stenger. Simultaneous multislice excitation by parallel transmission. Magnetic Resonance in Medicine, 71(4):1416-27, 2014.

[168] William S. Price. Pulsed-field gradient nuclear magnetic resonance as a tool for studying translational diffusion: Part 1. basic theory. Concepts in Magnetic Resonance, 9(5):299-336, 1997.

[169] E. M. Purcell, H. C. Torrey, and R. V. Pound. Resonance absorption by nuclear magnetic moments in a solid. Phys. Rev., 69:37-38, Jan 1946. doi: 10.1103/PhysRev.69.37. 
[170] Mekala R. Raman, Yunhong Shu, Timothy G. Lesnick, Clifford R. Jack, and Kejal Kantarci. Regional t1 relaxation time constants in ex vivo human brain: Longitudinal effects of formalin exposure. Magnetic Resonance in Medicine, 77(2):774-778, 2017.

[171] V. Rieke and K. Butts Pauly. Mr thermometry. Journal of Magnetic Resonance Imaging, 27(2):376-390, 2008.

[172] H. Risken. The Fokker-Planck equation. Methods of solution and applications. 2nd edition edition, 1989.

[173] A. Roebroeck, R. Galuske, E. Formisano, O. Chiry, H. Bratzke, I. Ronen, D. S. Kim, and R. Goebel. High-resolution diffusion tensor imaging and tractography of the human optic chiasm at 9.4 t. Neuroimage, 39(1):157-168, 2008.

[174] A. Roebroeck, S. Sengupta, M. Bastiani, S. Schillak, B. Tramm, M. Waks, A. Lataster, A. Herrler, D. H. Tse, and B. A. Poser. High resolution mri neuroanatomy of the whole human brain post mortem with a specialized 9.4t rf-coil. In Proceedings of the 2015 Organization for Human Brain Mapping annual meeting, 2015.

[175] A. Roebroeck, K. L. Miller, and M. Aggarwal. Ex vivo diffusion mri of the human brain: Technical challenges and recent advances. NMR Biomed, page e3941, 2018.

[176] H. Schomberg. Off-resonance correction of mr images. IEEE Transactions on Medical Imaging, 18(6):481-495, 1999.

[177] B. Schutz. A First Course in General Relativity. Cambridge University Press, 2009.

[178] A. Seehaus, A. Roebroeck, M. Bastiani, L. Fonseca, H. Bratzke, N. Lori, A. Vilanova, R. Goebel, and R. Galuske. Histological validation of high-resolution dti in human post mortem tissue. Front Neuroanat, 9:98, 2015.

[179] A. K. Seehaus, A. Roebroeck, O. Chiry, D. S. Kim, I. Ronen, H. Bratzke, R. Goebel, and R. A. Galuske. Histological validation 
of dw-mri tractography in human postmortem tissue. Cereb Cortex, 23(2):442-450, 2013.

[180] S. Sengupta, F. J. Fritz, R. L. Harms, S. Hildebrand, D. H. Y. Tse, B. A. Poser, R. Goebel, and A. Roebroeck. High resolution anatomical and quantitative mri of the entire human occipital lobe ex vivo at 9.4t. Neuroimage, 168:162-171, 2018.

[181] K. Setsompop, L. L. Wald, V. Alagappan, B. A. Gagoski, and E. Adalsteinsson. Magnitude least squares optimization for parallel radio frequency excitation design demonstrated at 7 tesla with eight channels. Magnetic Resonance in Medicine, 59(4):908-915, 2008.

[182] K. Setsompop, B. A. Gagoski, J. R. Polimeni, T. Witzel, V. J. Wedeen, and L. L. Wald. Blipped-controlled aliasing in parallel imaging for simultaneous multislice echo planar imaging with reduced g-factor penalty. Magnetic Resonance in Medicine, 67(5):1210-24, 2012.

[183] A. Shatil, K. Matsuda, and C. Figley. A method for whole brain ex vivo magnetic resonance imaging with minimal susceptibility artifacts. Frontiers in Neurology, 7:208, 2016.

[184] T. M. Shepherd, J. J. Flint, P. E. Thelwall, G. J. Stanisz, T. H. Mareci, A. T. Yachnis, and S. J. Blackband. Postmortem interval alters the water relaxation and diffusion properties of rat nervous tissueimplications for mri studies of human autopsy samples. Neuroimage, 44(3):820-826, 2009.

[185] T. M. Shepherd, P. E. Thelwall, G. J. Stanisz, and S. J. Blackband. Aldehyde fixative solutions alter the water relaxation and diffusion properties of nervous tissue. Magnetic Resonance in Medicine, 62 (1):26-34, 2009.

[186] S. N. Sotiropoulos, S. Jbabdi, J. L. Andersson, M. W. Woolrich, K. Ugurbil, and T. E. Behrens. Rubix: combining spatial resolutions for bayesian inference of crossing fibers in diffusion mri. IEEE Trans Med Imaging, 32(6):969-982, 2013.

[187] S. N. Sotiropoulos, S. Moeller, S. Jbabdi, J. Xu, J. L. Andersson, E. J. Auerbach, E. Yacoub, D. Feinberg, K. Setsompop, L. L. Wald, T. E. J. 
Behrens, K. Ugurbil, and C. Lenglet. Effects of image reconstruction on fiber orientation mapping from multichannel diffusion mri: Reducing the noise floor using sense. Magnetic Resonance in Medicine, 70(6):1682-1689, 2013.

[188] Olaf Sporns, Giulio Tononi, and Rolf KÃथtter. The human connectome: A structural description of the human brain. PLOS Computational Biology, 1(4), 09 2005. doi: 10.1371/journal.pcbi.0010042.

[189] S. St-Jean, P. Coupe, and M. Descoteaux. Non local spatial and angular matching: Enabling higher spatial resolution diffusion mri datasets through adaptive denoising. Med Image Anal, 32:115-130, 2016.

[190] Greg J. Stanisz, Ewa E. Odrobina, Joseph Pun, Michael Escaravage, Simon J. Graham, Michael J. Bronskill, and R. Mark Henkelman. $\mathrm{T} 1$, t2 relaxation and magnetization transfer in tissue at 3t. Magnetic Resonance in Medicine, 54(3):507-512, 2005.

[191] E. O. Stejskal. Use of spin echoes in a pulsed magnetic-field gradient to study anisotropic, restricted diffusion and flow. The Journal of Chemical Physics, 43(10):3597-3603, 1965.

[192] E. O. Stejskal and J. E. Tanner. Spin diffusion measurements: Spin echoes in the presence of a time-dependent field gradient. The Journal of Chemical Physics, 42(1):288-292, 1965.

[193] A. J. Steven, J. Zhuo, and E. R. Melhem. Diffusion kurtosis imaging: An emerging technique for evaluating the microstructural environment of the brain. American Journal of Roentgenology, 202(1): 26-33, 2013.

[194] N. Stikov, J. Campbell, M. Boudreau, S. Narayanan, T. Stroh, S. Nuara, J. Novek, S. Frey, Ming-Kay Ho, B. Bedell, and B. Pike. In vivo histology of the myelin g-ratio. In Proceedings of the $2014 \mathrm{Or}$ ganization for Human Brain Mapping annual meeting, page 2249.

[195] N. Stikov, J. Campbell, T. Stroh, M. Lavelee, S. Frey, J. Novek, S. Nuara, M-K. Ho, B. Bedell, F. Dougherty, I. Leppert, M. Boudreau, S. Narayanan, T. Duval, J. Cohen-Adad, P-A. Picard, 
A. Gasecka, D. Cote, and G. Pike. In vivo histology of the myelin g-ratio with magnetic resonance imaging. NeuroImage, 118:397 $405,2015$.

[196] C. Stuber, M. Morawski, A. Schafer, C. Labadie, M. Wahnert, C. Leuze, M. Streicher, N. Barapatre, K. Reimann, S. Geyer, D. Spemann, and R. Turner. Myelin and iron concentration in the human brain: a quantitative study of mri contrast. Neuroimage, $93 \mathrm{Pt} 1$ : 95-106, 2014.

[197] S. W. Sun, J. J. Neil, H. F. Liang, Y. Y. He, R. E. Schmidt, C. Y. $\mathrm{Hsu}$, and S. K. Song. Formalin fixation alters water diffusion coefficient magnitude but not anisotropy in infarcted brain. Magnetic Resonance in Medicine, 53(6):1447-1451, 2005.

[198] F; Cheng-JY; Uecker M; Lustig M Tamir, Jl; Ong. Generalized magnetic resonance image reconstruction using the berkeley advanced reconstruction toolbox. In ISMRM Workshop on Data Sampling and Image Reconstruction.

[199] U.S.; Witzel-T.; Jones D.K. Tax, C.M.W; Rudrapatna. Disentangling in two dimensions in the living human brain: Feasibilty of relaxometry-diffusometry using ultra-strong gradients. In ISMRM 25th Annual Meeting \& Exhibition, 2017.

[200] W. M. Teeuwisse, W. M. Brink, and A. G. Webb. Quantitative assessment of the effects of high-permittivity pads in 7 tesla mri of the brain. Magnetic Resonance in Medicine, 67(5):1285-93, 2012.

[201] Piotr Tekely. Malcolm h. levitt. spin dynamics: basics of nuclear magnetic resonance. Magnetic Resonance in Chemistry, 40(12):800$800,2002$.

[202] B. Tendler, S. Foxley, M. Cottaar, S. Jbabdi, and K. Miller. Modeling an equivalent $b$-value in diffusion-weighted steady-state free precession. Magnetic Resonance in Medicine, 84(2):873-884, 2020.

[203] C. Thomas, Frank Q. Ye, M. O. Irfanoglu, P. Modi, K. S. Saleem, D. A. Leopold, and C. Pierpaoli. Anatomical accuracy of brain connections derived from diffusion mri tractography is inherently limited. Pro- 
ceedings of the National Academy of Sciences, 111(46):16574-16579, 2014.

[204] H. C. Torrey. Bloch Equations with Diffusion Terms. Physical Review, 104:563-565, nov 1956.

[205] J. D. Tournier, R. Smith, D. Raffelt, R. Tabbara, T. Dhollander, M. Pietsch, D. Christiaens, B. Jeurissen, C. H. Yeh, and A. Connelly. Mrtrix3: A fast, flexible and open software framework for medical image processing and visualisation. Neuroimage, 202:116137, 2019.

[206] J.D. Tournier, F. Calamante, and A. Connelly. Robust determination of the fibre orientation distribution in diffusion mri: Non-negativity constrained super-resolved spherical deconvolution. NeuroImage, 35 (4):1459 - 1472, 2007.

[207] C. Triantafyllou, J. R. Polimeni, and L. L. Wald. Physiological noise and signal-to-noise ratio in fmri with multi-channel array coils. NeuroImage, 55(2):597-606, 2011.

[208] D. H. Tse, C. J. Wiggins, D. Ivanov, D. Brenner, J. Hoffmann, C. Mirkes, G. Shajan, K. Scheffler, K. Uludag, and B. A. Poser. Volumetric imaging with homogenised excitation and static field at 9.4 t. Magma , 29(3):333-345, 2016.

[209] M. Uecker, P. Lai, M. J. Murphy, P. Virtue, M. Elad, J. M. Pauly, S. S. Vasanawala, and M. Lustig. Espirit-an eigenvalue approach to autocalibrating parallel mri: where sense meets grappa. Magnetic Resonance in Medicine, 71(3):990-1001, 2014.

[210] M. Utz and R. Monazami. Nuclear magnetic resonance in microfluidic environments using inductively coupled radiofrequency resonators. Journal of magnetic resonance, 198(1):132-136, 2009.

[211] K. Uğurbil. Imaging at ultrahigh magnetic fields: History, challenges, and solutions. NeuroImage, 168:7 - 32, 2018.

[212] David C. Van Essen, Saad Jbabdi, Stamatios N. Sotiropoulos, Charles Chen, Krikor Dikranian, Tim Coalson, John Harwell, Timothy E. J. Behrens, and Matthew F. Glasser. Chapter 16 - Mapping 
Connections in Humans and Non-Human Primates: Aspirations and Challenges for Diffusion Imaging, pages 337-358. San Diego, 2014.

[213] P. Van Gelderen, D. Despres, and C. Moonen. Evaluation of restricted diffusion in cylinders. phosphocreatine in rabbit leg muscle. Journal of Magnetic Resonance - Series B, 103(3):255-260, 1994.

[214] J. Veraart, E. Fieremans, I. Jelescu, F. Knoll, and D. Novikov. Gibbs ringing in diffusion mri. Magnetic Resonance in Medicine, 76(1): 301-314, 2016.

[215] J. Veraart, D. S. Novikov, D. Christiaens, B. Ades-Aron, J. Sijbers, and E. Fieremans. Denoising of diffusion mri using random matrix theory. Neuroimage, 142:394-406, 2016.

[216] J. Veraart, D. S. Novikov, and E. Fieremans. Te dependent diffusion imaging (teddi) distinguishes between compartmental $\mathrm{t} 2$ relaxation times. Neuroimage, 182:360-369, 2018.

[217] O. Vogt. Die myeloarchitektonische felderung des menschlichen stirnhirns. J Psychol Neurol, 15:221-232, 1910.

[218] C. S. von Bartheld, J. Bahney, and S. Herculano-Houzel. The search for true numbers of neurons and glial cells in the human brain: A review of 150 years of cell counting. Journal of Comparative Neurology, 524(18):3865-3895, 2016.

[219] S. B. Vos, C. M. W. Tax, P. R. Luijten, S. Ourselin, A. Leemans, and M. Froeling. The importance of correcting for signal drift in diffusion mri. Magnetic Resonance in Medicine, 77(1):285-299, 2017.

[220] A. T. Vu, E. Auerbach, C. Lenglet, S. Moeller, S. N. Sotiropoulos, S. Jbabdi, J. Andersson, E. Yacoub, and K. Ugurbil. High resolution whole brain diffusion imaging at $7 \mathrm{t}$ for the human connectome project. Neuroimage, 122:318-331, 2015.

[221] T. Wallace, O. Afacan, M. Waszak, T. Kober, and S. Warfield. Head motion measurement and correction using fid navigators. Magnetic Resonance in Medicine, 81(1):258-274, 2019. 
[222] F. Wang, Z. Dong, T. G. Reese, B. Bilgic, M. Katherine Manhard, J. Chen, J. R. Polimeni, L. L. Wald, and K. Setsompop. Echo planar time-resolved imaging (epti). Magnetic Resonance in Medicine, 81 (6):3599-3615, 2019.

[223] Y. Wang, P. van Gelderen, J.A. de Zwart, and J.H. Duyn. B0-field dependence of mri t1 relaxation in human brain. NeuroImage, 213: $116700,2020$.

[224] P E Watson, I D Watson, and R D Batt. Total body water volumes for adult males and females estimated from simple anthropometric measurements. The American Journal of Clinical Nutrition, 33(1): 27-39, 1980.

[225] N. Weiskopf, J. Suckling, G. Williams, M. M. Correia, B. Inkster, R. Tait, C. Ooi, E. T. Bullmore, and A. Lutti. Quantitative multiparameter mapping of $\mathrm{r} 1, \mathrm{pd}(*), \mathrm{mt}$, and $\mathrm{r} 2\left(^{*}\right)$ at $3 \mathrm{t}$ : a multi-center validation. Front Neurosci, 7:95, 2013.

[226] C. F. Westin, S. E. Maier, H. Mamata, A. Nabavi, F. A. Jolesz, and R. Kikinis. Processing and visualization for diffusion tensor mri. Med Image Anal, 6(2):93-108, 2002.

[227] S. Wharton and R. Bowtell. Fiber orientation-dependent white matter contrast in gradient echo mri. Proceedings of the National Academy of Sciences, 109(45):18559-18564, 2012.

[228] N. White, C. Roddey, A. Shankaranarayanan, E. Han, D. Rettmann, J. Santos, J. Kuperman, and A. Dale. Promo: Real-time prospective motion correction in mri using image-based tracking. Magnetic Resonance in Medicine, 63(1):91-105, 2010.

[229] R. Wiest and R. Beisteiner. Recent developments in imaging of epilepsy. Current opinion in Neurology, 32(4):530-538, 2019.

[230] M L Winkler, D A Ortendahl, T C Mills, L E Crooks, P E Sheldon, L Kaufman, and D M Kramer. Characteristics of partial flip angle and gradient reversal mr imaging. Radiology, 166(1):17-26, 1988.

[231] Q.; Poetter-C.; Zhu K.; Middione M.; Kerr A.B.; McNab J.A.; 
Dougherty R. Wu, H.; Tian. Whole brain inversion recovery diffusion weighted imaging using slice-shuffled acquisition. In ISMRM 25th Annual Meeting \& Exhibition, 2017.

[232] X. Wu, S. Schmitter, E. J. Auerbach, S. Moeller, K. Ugurbil, and P. F. Van de Moortele. Simultaneous multislice multiband parallel radiofrequency excitation with independent slice-specific transmit b1 homogenization. Magnetic Resonance in Medicine, 70(3):630-8, 2013.

[233] U. Yarach, C. Luengviriya, D. Stucht, F. Godenschweger, P. Schulze, and O. Speck. Correction of b0-induced geometric distortion variations in prospective motion correction for $7 \mathrm{t}$ mri. Magnetic Resonance Materials in Physics, Biology and Medicine volume, 29:319$332,2016$.

[234] V. L. Yarnykh. Actual flip-angle imaging in the pulsed steady state: a method for rapid three-dimensional mapping of the transmitted radiofrequency field. Magnetic Resonance in Medicine, 57(1):192200, 2007.

[235] H. Zhang, T. B. Dyrby, and D. C. Alexander. Axon diameter mapping in crossing fibers with diffusion mri. Med Image Comput Comput Assist Interv, 14(Pt 2):82-89, 2011.

[236] H. Zhang, P.L. Hubbard, G.J.M. Parker, and D.C. Alexander. Axon diameter mapping in the presence of orientation dispersion with diffusion mri. NeuroImage, 56(3):1301 - 1315, 2011.

[237] H. Zhang, T. Schneider, C. A. Wheeler-Kingshott, and D. C. Alexander. Noddi: practical in vivo neurite orientation dispersion and density imaging of the human brain. Neuroimage, 61(4):1000-1016, 2012 .

[238] Y. Zhang, S.A. Wells, and D. Hernando. Stimulated echo based mapping (stem) of $\mathrm{t} 1, \mathrm{t} 2$, and apparent diffusion coefficient: validation and protocol optimization. Magnetic Resonance in Medicine, 81(1): 167-181, 2019. 